Florida International University FIU Digital Commons

$11-8-2013$

\title{
Field Development and Performance Evaluation of a Constructed Wetland System in the Tropics
}

Adelaide Pereira Hummel

Florida International University, adelaideph@hotmail.com

DOI: $10.25148 /$ etd.FI14040897

Follow this and additional works at: https://digitalcommons.fiu.edu/etd

Part of the Civil Engineering Commons

\section{Recommended Citation}

Hummel, Adelaide Pereira, "Field Development and Performance Evaluation of a Constructed Wetland System in the Tropics" (2013). FIU Electronic Theses and Dissertations. 1218.

https://digitalcommons.fiu.edu/etd/1218 


\section{FLORIDA INTERNATIONAL UNIVERSITY}

Miami, Florida

\section{FIELD DEVELOPMENT AND PERFORMANCE EVALUATION OF A CONSTRUCTED WETLAND SYSTEM IN THE TROPICS}

A dissertation submitted in partial fulfillment of

the requirements for the degree of

DOCTOR OF PHILOSOPHY

in

CIVIL ENGINEERING

by

Adelaide Pereira Hummel

2014 
To: Dean Amir Mirmiran

College of Engineering and Computing

This dissertation, written by Adelaide Pereira Hummel, and entitled Field Development and Performance Evaluation of a Constructed Wetland in the Tropics, having been approved in respect to style and intellectual content, is referred to you for judgment.

We have read this dissertation and recommend that it be approved.

Omar I. Abdul-Aziz

Yelena Katsenovich

Shonali Laha

Assefa Melesse

Hector R. Fuentes, Major Professor

Date of Defense: November 8, 2013

The dissertation of Adelaide Pereira Hummel is approved.

Dean Amir Mirmiran

College of Engineering and Computing

Dean Lakshmi N. Reddi

University Graduate School

Florida International University, 2014 
C Copyright 2014 by Adelaide Pereira Hummel

All rights reserved 


\section{DEDICATION}

I dedicate this dissertation to my husband, Andre, and my children, Nicolas and Sophia, with my deepest expression of love and appreciation for their sacrifices made during this long journey and I am very thankful for having you in my life. I also dedicate this dissertation to my parents and my aunt Yolanda for their words of encouragement and for their example of perseverance; and to my nephews Lucas, Alexandre Junior, Felipe and Eduardo, and my nieces Laura and Juliana; I hope to be inspirational to your lives. 


\section{ACKNOWLEDGMENTS}

I would like to thank the Applied Research Center (ARC) at Florida International University (FIU) for the opportunity to conduct this study through a graduate research assistantship. This research project was funded by the U.S. Department of Defense (U.S. DOD) Office of the Assistant Secretary of the Army for Installations and Environment (OASA I\&E). I shall mention my former Project Manager, Lawrence Moss (Larry) - it was a great pleasure working with him and learning from him. Larry made our trips to El Salvador very productive and enjoyable.

I would like to express my deepest gratitude to my advisor, Dr. Hector R. Fuentes, for his excellent guidance, caring, patience, friendship, and was always willing to help, giving his best advices, and always going way beyond his advisory role.

To Drs. Yelena Katsenovich, Shonali Laha, Assefa Melesse, and Omar I. Abdul-Aziz, I thank you for your support and advice as members of my dissertation committee. I take this opportunity to thank the staff in the Civil Engineering Department - Haydee Caneda - for her great help with the required paperwork along these years. I also would like to express my sincere gratitude to Carlton $\mathrm{Ng}$ and Claudia Cardona for being my hands and angels during the final stage. 


\section{DISCLAIMER}

This dissertation proposal was prepared as a part of FIU academic curricula, with data collected under a project sponsored by an agency of the United States Government. Neither the United States of America nor any agency thereof, nor any of their employees, nor any of its contractors, subcontractors, nor their employees make any warranty, express or implied, or assumes any legal liability or responsibility for the accuracy, completeness, or usefulness of any information, apparatus, product, or process disclosed, or represents that's its use would not infringe upon privately owned rights. The views and opinions of authors expressed herein do not necessarily state or reflect those of the United States government or nay agency thereof. 


\title{
ABSTRACT OF THE DISSERTATION \\ FIELD DEVELOPMENT AND PERFORMANCE EVALUATION OF A CONSTRUCTED WETLAND IN THE TROPICS
}

\author{
By \\ Adelaide Pereira Hummel \\ Florida International University, 2014 \\ Miami, Florida \\ Professor Hector R. Fuentes, Major Professor
}

This study is part of a project that was conducted by Florida International University (FIU), which designed, built, and characterized a constructed wetland (CW) wastewater treatment system in El Salvador, Central America. This study focuses on the detailed performance of a field-scale $\mathrm{CW}$ in the tropics, consisting of a subsurface flow wetland (SSF) and a surface flow wetland (SF). The SSF had a total area of 151.2 sq. m., filled with gravel and planted with Phragmites, Thalia, and Brachiaria, along three independent cells operating with a flow in parallel and receiving the influent domestic wastewater from a facultative lagoon and preceded the SF. The SF was a shallow open basin containing alternating regions of open water (195 sq. m. total) with submerged aquatic plants (Elodea) and regions of shallower water (605 sq. m. total) filled with emergent wetland vegetation (Typha, Thalia, and Cyperus).

The design, construction, startup and operation phases of the field scale $\mathrm{CW}$ in that tropical setting are thus described with detail, including both SSF and SF 
characterization of influent and effluents. The SSF average hydraulic detention times during the wet and dry season were 2 days \pm 0.9 days and 4 days \pm 0.4 days, respectively; and the SF average hydraulic detention times during the wet and dry season were 20 days \pm 11.1 days and 77 days \pm 19.5 days, respectively. Brachiaria/Cyperus presented better results during the wet season with average $\mathrm{BOD}_{5}$ residuals of $36 \% \pm 25 \%$, and Thalia/Thalia during the dry season with average $\mathrm{BOD}_{5}$ residuals of $33 \% \pm 22 \%$. Phragmites/Typha presented better results during both seasons with average TSS residuals of $2 \% \pm 3 \%$ (wet season), and $2 \% \pm 2 \%$ (dry season). Residuals are also presented for COD, Oil \& Grease, Total Fecal Coliform, Total Phosphorus, and Total Nitrogen. In addition, an assessment of the empirical models used in the design of the system is completed, having the EPA methodology as the preferred for $\mathrm{BOD}_{5}$ removal and three methodologies for TSS removal under tropical climate conditions. A comparison of the differences in treatment associated with each one of the selected plants and their combinations is also discussed. In summary, results strongly suggest that the $\mathrm{CW}$ system can effectively reduce contaminants in wastewater to levels that are comparable with the objective levels (i.e., secondary treatment levels). 


\section{TABLE OF CONTENTS}

CHAPTER

PAGE

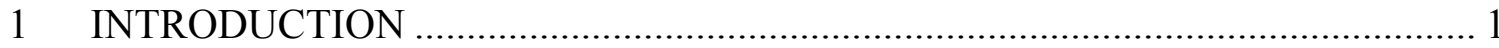

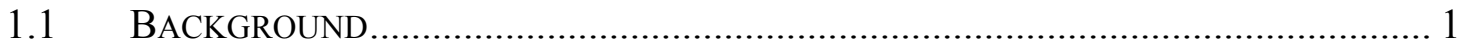

1.1.1 Constructed Wetland Design ...................................................................... 7

1.1.2 Role of Plants in Constructed Wetlands ………….................................... 18

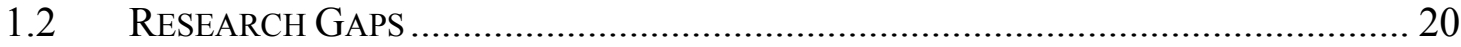

1.3 CENTRAL HYPOTHESIS AND RESEARCH OBJECTIVES ........................................ 21

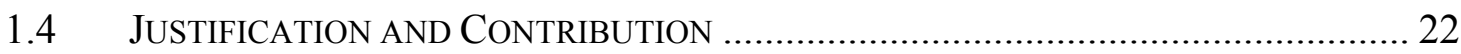

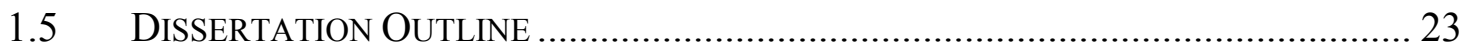

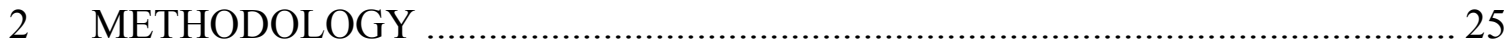

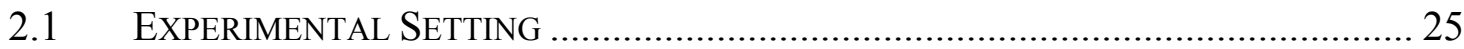

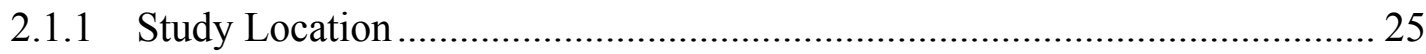

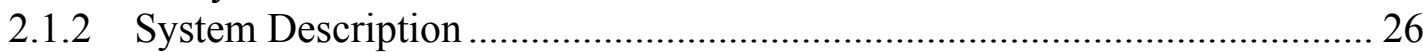

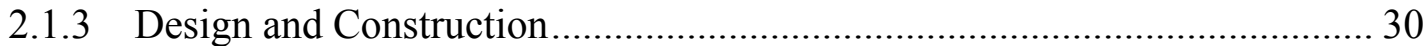

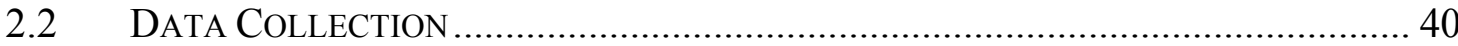

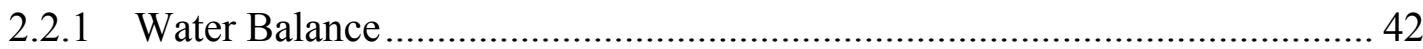

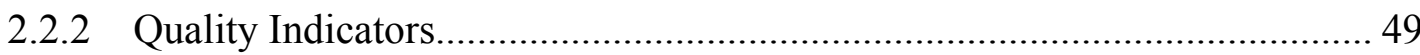

2.2.3 Field Parameters................................................................................. 49

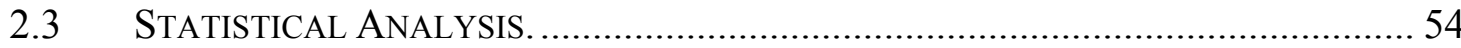

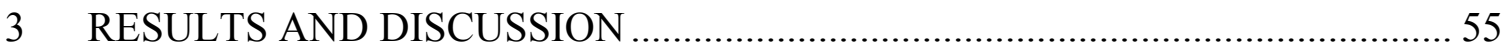

3.1 INFLUENT AND EFFLUENT CHARACTERIZATION............................................5 55

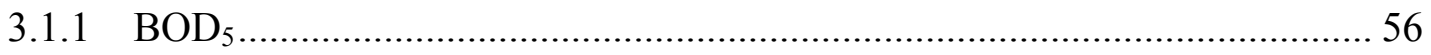

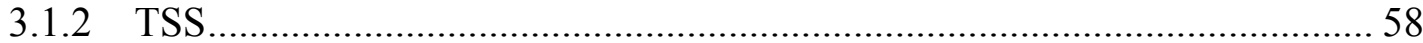

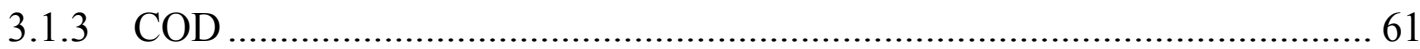

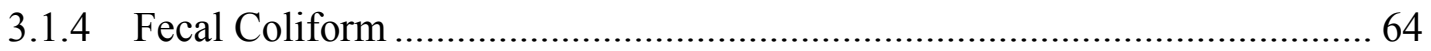

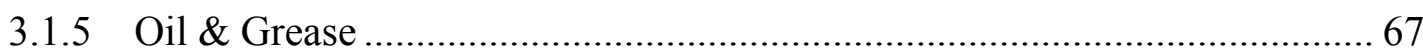

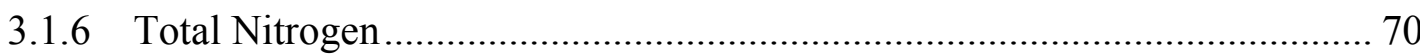

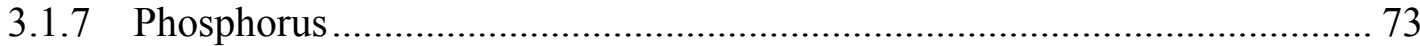

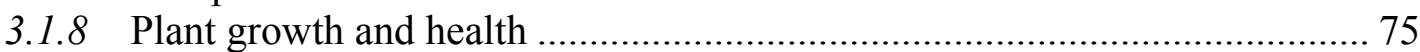

3.2 COMPARATIVE ASSESSMENT OF MODELS USED DURING DESIGN........................ 79

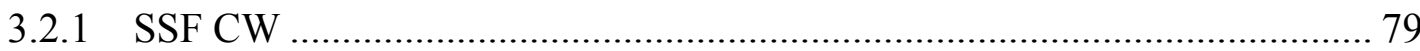

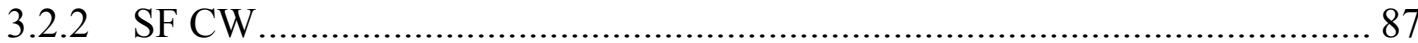

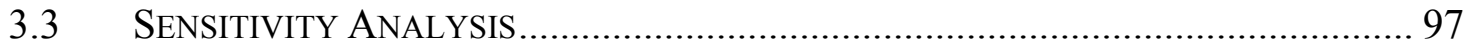

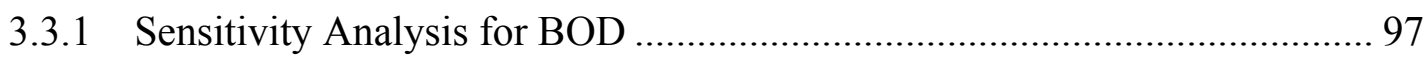

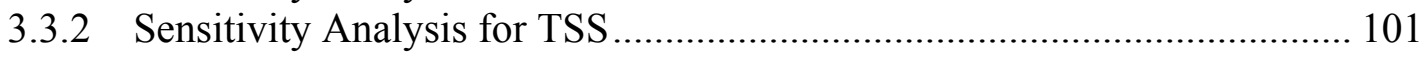

4 CONCLUSIONS, LIMITATIONS AND RECOMMENDATIONS ……….......... 105

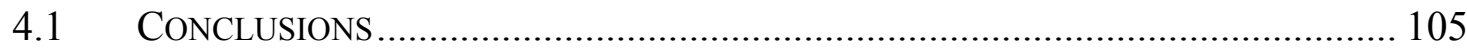

4.2 LIMITATIONS AND RECOMMENDATIONS ................................................... 107 


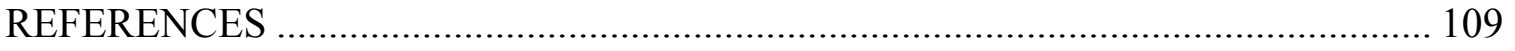

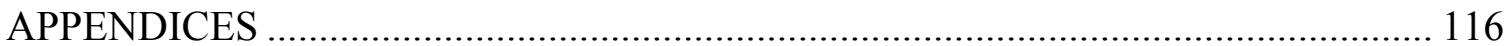

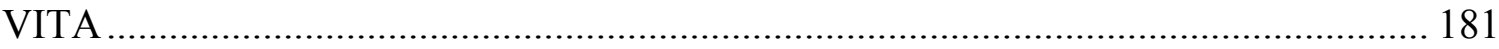




\section{LIST OF TABLES}

TABLE

PAGE

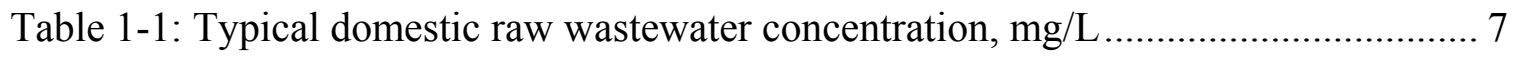

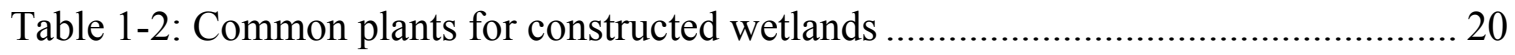

Table 2-1: Design Flow Rates Used in Method Comparison ........................................ 32

Table 2-2: Different Design Method Results Summary ............................................. 34

Table 2-3: Results of Design Calculations for BOD Removal in SSF CW .................... 35

Table 2-4: Results of Design Calculations for Total N Removal in SF CW ................... 36

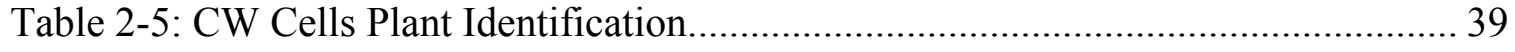

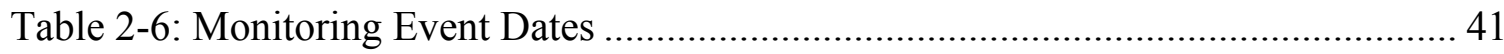

Table 2-7: Water Quality Analytical Parameters (by FUSADES) ................................ 42

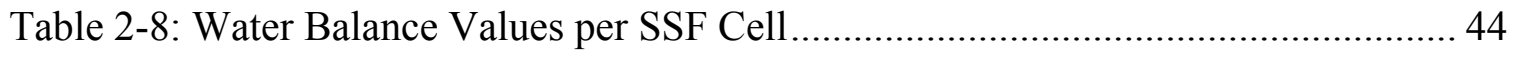

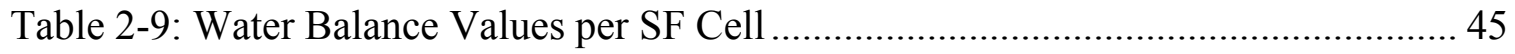

Table 2-10: Seasonal Water Balance Values per SSF Cell.......................................... 46

Table 2-11: Seasonal Water Balance Values per SF Cell ........................................... 47

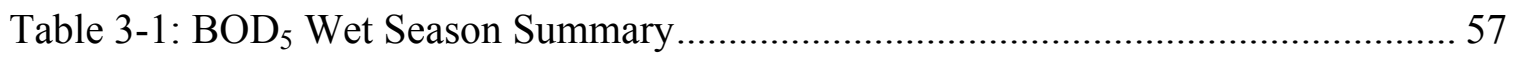

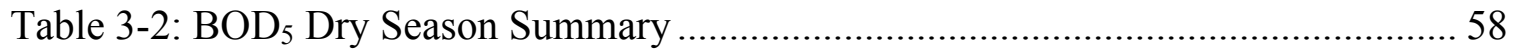

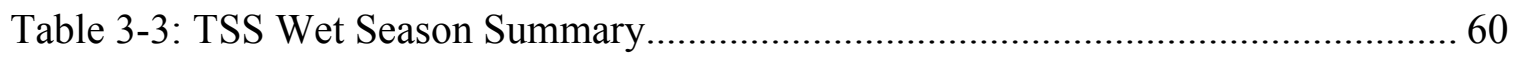

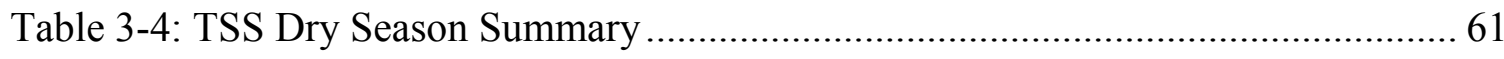

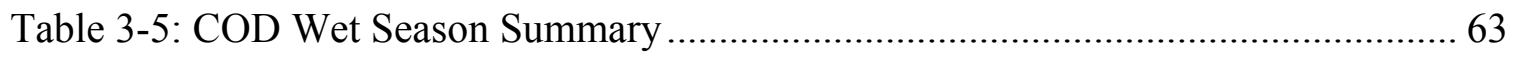

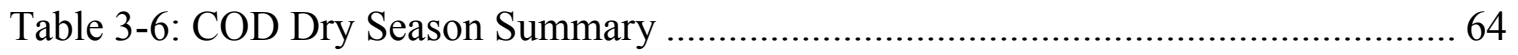




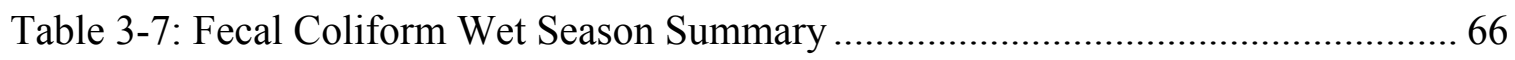

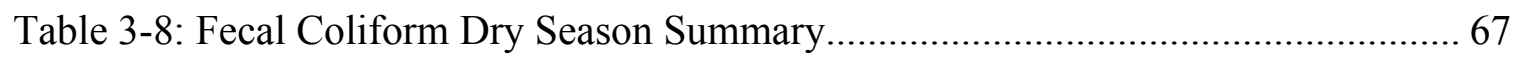

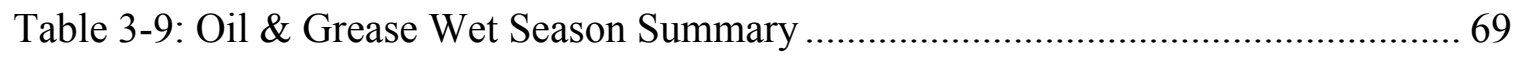

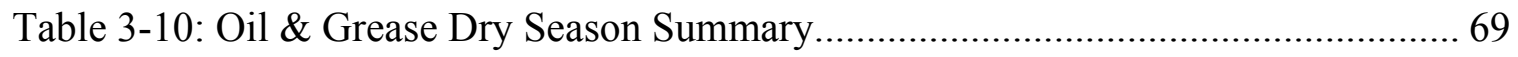

Table 3-11: Total Nitrogen Wet Season Summary ................................................. 71

Table 3-12: Total Nitrogen Dry Season Summary ….............................................. 72

Table 3-13: Phosphorus Wet Season Summary ........................................................ 74

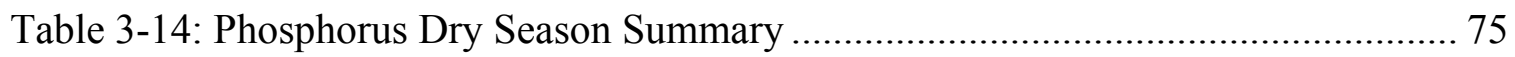

Table 3-15: Nitrogen and Phosphorus Dry Plant Composition ................................... 77

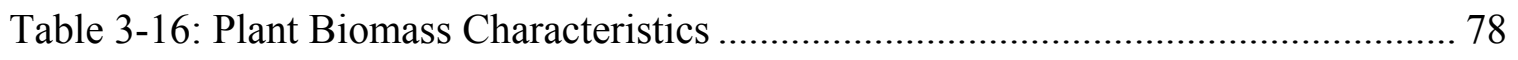

Table 3-17: Sensitivity Analysis Minimum and Maximum Values Used for BOD ......... 98

Table 3-18: Sensitivity Analysis Minimum and Maximum Values Used for TSS......... 102 


\section{LIST OF FIGURES}

FIGURE

PAGE

Figure 1-1: Subsurface Flow Wetland ................................................................... 2

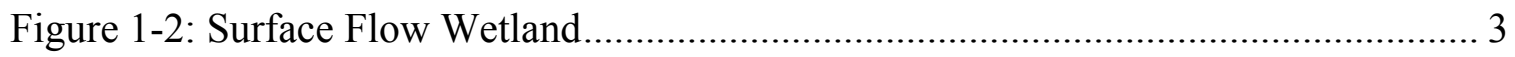

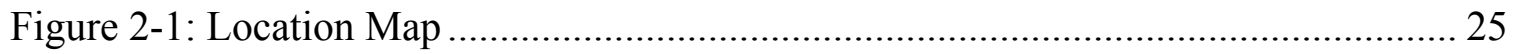

Figure 2-2: Facultative Lagoon System Schematic (Prior to CW System Construction). 26

Figure 2-3: Complete Treatment System Schematic (new) ......................................... 27

Figure 2-4: Site Plan with Constructed Wetland System......................................... 28

Figure 2-5: Precipitation, Evaporation, Temperature ............................................... 33

Figure 2-6: SSF and SF CW Layout, Plants Distribution, and Monitoring Points ........... 39

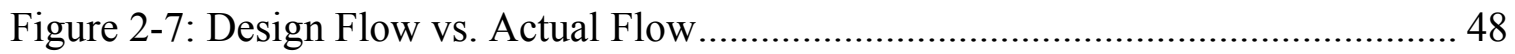

Figure 2-8: Field Parameter - Average Temperature ….......................................... 51

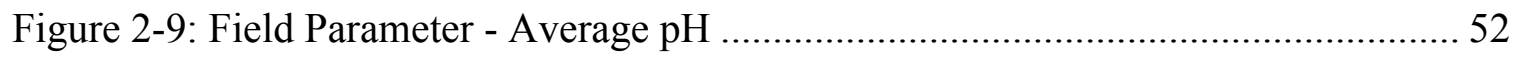

Figure 2-10: Field Parameter - Average ORP....................................................... 52

Figure 2-11: Field Parameter - Average DO............................................................. 53

Figure 2-12: Field Parameter - Average Conductivity ............................................. 53

Figure 3-1: $\mathrm{BOD}_{5}$ Influent vs. Effluent Concentrations ......................................... 56

Figure 3-2: TSS Influent vs. Effluent Concentrations ........................................... 59

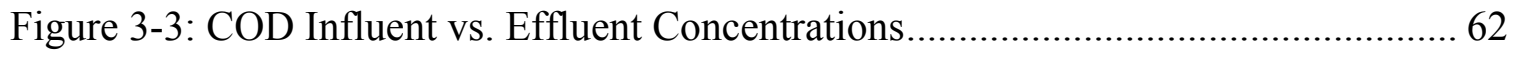

Figure 3-4: Fecal Coliform Influent vs. Effluent Concentrations ............................... 65

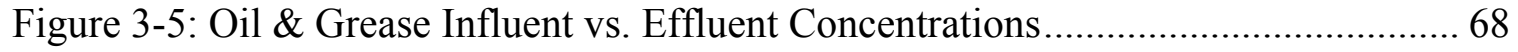

Figure 3-6: Total Nitrogen Influent vs. Effluent Concentrations ................................ 70 
Figure 3-7: Phosphorus Influent vs. Effluent Concentrations 73

Figure 3-8: $\mathrm{BOD}_{5}$ Design Target vs. Actual Effluent Concentrations - Kadlec \& Knight, Campbell \& Ogden, and Reed et.al (SSF CW). 82

Figure 3-9: $\mathrm{BOD}_{5}$ Design Target vs. Actual Effluent Concentrations - EPA (SSF CW) 83

Figure 3-10: TSS Design Target vs. Actual Effluent Concentrations - Kadlec \& Knight,

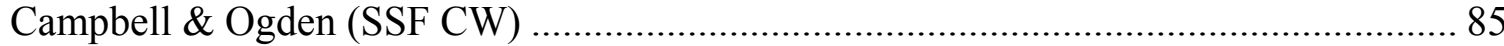

Figure 3-11: TSS Design Target vs. Actual Effluent Concentrations - Kadlec \& Knight, Campbell \& Ogden (Closer View) (SSF CW) ............................................................. 86

Figure 3-12: TSS Design Target vs. Actual Effluent Concentrations - EPA (SSF CW). 87

Figure 3-13: $\mathrm{BOD}_{5}$ Design Target vs. Actual Effluent Concentrations - Kadlec \& Knight, Campbell \& Ogden, and Reed et.al (SF CW) ....................................................... 90

Figure 3-14: $\mathrm{BOD}_{5}$ Design Target vs. Actual Effluent Concentrations - EPA (SF CW) 91

Figure 3-15: TSS Design Target vs. Actual Effluent Concentrations - Kadlec \& Knight,

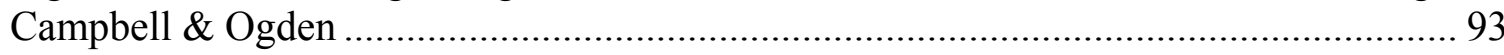

Figure 3-16: TSS Design Target vs. Actual Effluent Concentrations - Kadlec \& Knight, Campbell \& Ogden (Closer View) ..................................................................... 94

Figure 3-17: TSS Design Target vs. Actual Effluent Concentrations - EPA................... 95

Figure 3-18: Total Nitrogen Design Target vs. Actual Effluent Concentrations - Kadlec

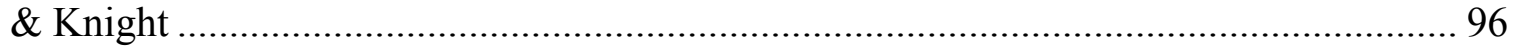

Figure 3-19: SSF Sensitivity Analysis for Areal Rate Constant (BOD)........................ 99

Figure 3-20: SSF Sensitivity Analysis for Net Flow (BOD) ...................................... 100

Figure 3-21: SSF Sensitivity Analysis for Background Concentration (BOD) .............. 100

Figure 3-22: SSF Sensitivity Analysis for Areal Rate Constant (TSS) ......................... 103

Figure 3-23: SSF Sensitivity Analysis for Net Flow (TSS) ..................................... 103

Figure 3-24: SSF Sensitivity Analysis for Background Concentration (TSS) .............. 104 


\section{LIST OF ACRONYMS}
AVE
Average
BOD
Biological Oxygen Demand
CENTA
Centro Nacional de Tecnologia Agropecuaria y Forestal - El Salvador Agricultural Agency
$\mathrm{CO} 2$
Carbon Dioxide
COD
Chemical Oxygen Demand
DO
Dissolved Oxygen
EPA
Environmental Protection Agency
ET
Evapotranspiration
FC
Fecal Coliform
FIU
Florida International University
HDPE
High-density polyethylene
$\mathrm{k}$
Reaction Rate
n.d.
no date
N.D.
Not detected
NH4 as N
Ammonia Nitrogen 


$\begin{array}{ll}\text { NO2- as N } & \text { Nitrite Nitrogen } \\ \text { NO3- as N } & \text { Nitrate Nitrogen } \\ \text { NPDES } & \text { National Pollutant Discharge Elimination System } \\ \text { NRCS } & \text { Natural Resources Conservation Services } \\ \text { O\&G } & \text { Oil \& Grease } \\ \text { O\&M } & \text { Operation \& Maintenance } \\ \text { Org N } & \text { Organic Nitrogen } \\ \text { ORP } & \text { Oxidation Reduction Potential } \\ \text { P } & \text { Phosphorus } \\ \text { PR } & \text { Pump Runtime } \\ \text { PVC } & \text { Polyvinyl chloride } \\ \text { SACR } & \text { Salvadoran Army Cavalry Regiment } \\ & \text { Standard Deviation } \\ \text { SD } & \\ & \end{array}$


TKN

TSS

U.S.

USDA

USEPA

UV

WWTP
Total Kjeldahl Nitrogen

Total Suspended Solids

United States

U.S. Department of Agriculture

U.S. Environmental Protection Agency

Ultraviolet

Wastewater Treatment Plant 


\section{INTRODUCTION}

\subsection{Background}

Everyone generates wastewater. When people use water, it becomes wastewater that must be cleaned up before it is safely reused or returned to the environment. Water use should be done in ways that ensure sustainable supplies and quality for future generations.

Wastewater contains organic and inorganic substances, nutrients, such as nitrogen and phosphorus, and solids. Therefore, it is important to treat wastewater not only to ensure its beneficial reuse but also to protect human and environmental health.

Constructed wetlands are one type of wastewater treatment that imitates the natural environment. Constructed wetlands are typically easy to operate and maintain, low in operational and maintenances costs, and capable of effective treatment (Kadlec \& Knight, 1996); (Kadlec, et al., 2000); (Halverson, 2004) (Tsihrintzis, et al., 2007). Additional benefits are the provision of habitat for fish and wildlife and their intrinsic aesthetic appeal.

Constructed wetlands $(\mathrm{CW})$ are a competitive technology to treat a variety of wastewater. Their simpler operation and maintenance than more energy-intense technology, such as the conventional activated sludge, makes them ideal options for the treatment of domestic wastewaters in developing countries, especially when land is available and not costly. Constructed wetland systems are commonly classified in three types: a) subsurface flow; b) surface flow, and c) hybrid designs that combine both (Zhi \& Ji, 2012) (Vymazal, 2011). 
Subsurface flow constructed wetland (SSF CW) consists of a basin or channel with a seepage barrier, but the bed is filled with porous media, such as rock and gravel, as shown in Figure 1-1. The media supports the vegetation root structure. SSF CWs are designed to keep the water level below the top of the rock or gravel media. Bed depth for subsurface flow wetlands is generally less than $2 \mathrm{ft}(0.6 \mathrm{~m})$. Typical flow depths vary from 1.6 to $2.6 \mathrm{ft}(0.49$ to $0.79 \mathrm{~m})$. Typical hydraulic loading rates are between $0.8-8$ in/day ( 2 to $20 \mathrm{~cm} /$ day), which corresponds to a wetland of 4.7 to 47 acres per $\mathrm{mgd}$ of flow (0.5 to 5 ha per $1000 \mathrm{~m}^{3} /$ day of flow) (Halverson, 2004). SSF CWs tend to be more effective at filtering out solids and removing biological oxygen demand (BOD) per unit land area. Because the wastewater remains below the surface in these systems, there is less possibility for human or wildlife contact with wastewaters and less potential for insect infestation.

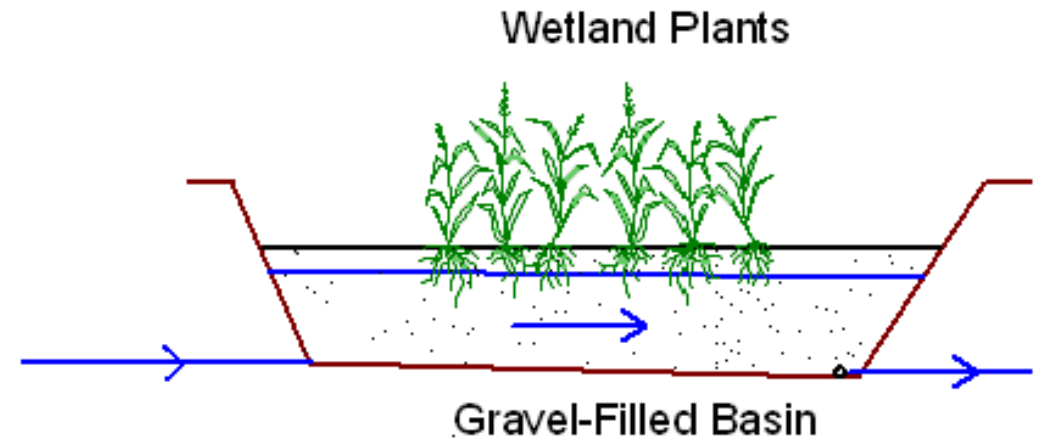

Figure 1-1: Subsurface Flow Wetland

Surface flow constructed wetland (SF CW), also called free surface flow wetland, consists of a basin or channels with a barrier layer to prevent seepage, soil to support rooted vegetation, and relatively shallow water flowing through the system, as shown 
Figure 1-2. The water flows primarily horizontally and above ground. Water movement in SF CW is complex. It varies in space and time because of the wetland vegetation and waste and it changes the inflow characteristics, and the stochastic nature of hydrologic events. SF CWs are densely vegetated and typically have water depths less than $1.3 \mathrm{ft}$ (0.4m). Typical hydraulic loading rates are between 0.3 and 2 in/day (0.7 and 5.0 $\mathrm{cm} /$ day), which corresponds to a wetland of 18.7 to 13.1 acres per mgd of flow (2 to 14 ha per $1000 \mathrm{~m}^{3} /$ day of flow). Typical flow depths for SF CWs are 0.3 to $2.0 \mathrm{ft}$ (0.09 to 0.06m) (Halverson, 2004). SF CWs are cheaper and easy to construct, operate and maintain, have higher wildlife habitat values and they offer a greater flow control than subsurface flow wetland. The main disadvantage is that they generally require more land than SSF CW systems.

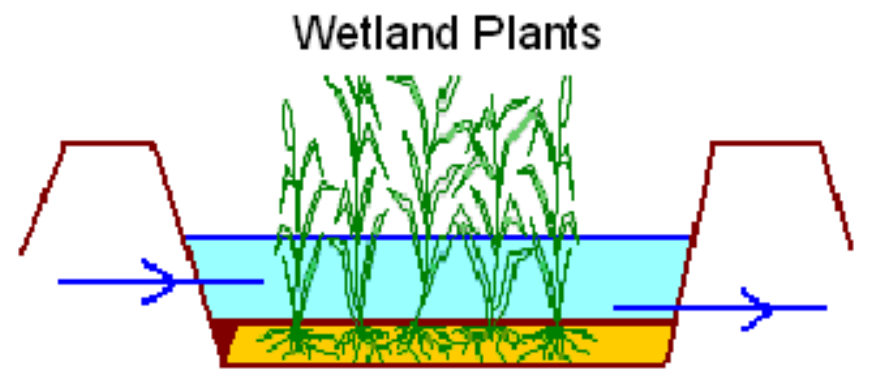

Figure 1-2: Surface Flow Wetland

CW studies shows that total suspended solids (TSS) loadings of less than $30 \mathrm{~kg} / \mathrm{d}$-ha or lower were able to consistently produce an effluent with less than $20 \mathrm{mg} / \mathrm{L}$ of TSS. Loadings less than $50 \mathrm{~kg} / \mathrm{d}$-ha produced an effluent with less than $30 \mathrm{mg} / \mathrm{L}$ TSS (U.S. EPA, 1999). Systems with open water zones were able to produce effluent with lower amounts of TSS than the ones that were fully vegetated. Wetland systems can 
significantly reduce constituents with removal efficiencies in the range of 70 to 90 percent for TSS (U.S. Army Corps of Engineers, 2003).

A study of 20 operating wetlands in U.S. that contained significant open-water zones indicated that SF CWs could be expected to yield effluent with less than $20 \mathrm{mg} / \mathrm{L} \mathrm{BOD}$ at mass loadings below $45 \mathrm{~kg} / \mathrm{d}$-ha (U.S. EPA, 1999). Fully vegetated systems will normally produce $50 \%$ more effluent BOD and TSS $(30 \mathrm{mg} / \mathrm{L})$ than wetlands with openwater areas at comparable loading rates.

The removal of nitrogen and phosphorous takes a limited place on CWs (U.S. EPA, 1999) (Mburu, et al., 2013). Nitrogen removal in the SSF CW system is relatively small since such system is usually anoxic and oxidation does not occur. Nitrogen removals in the literature range between 0.01 and $2.15 \mathrm{~g} \mathrm{~N} / \mathrm{m}^{2}$-day, with an average value of $0.048 \mathrm{~g}$ $\mathrm{N} / \mathrm{m}^{2}$-day (Vymazal, 2007).

The removal of phosphorous from the wastewater is limited to adsorption onto sediment and plant material, entrainment of phosphorous-containing solids into sediment and uptake into plant tissue (much of which is subsequently released as that tissue decomposes in the wetland) (Stefanakis \& Tsihrintzis, 2009). Large wetlands with long hydraulic retention times are needed to achieve significant phosphorous removal. A phosphorous loading of less than $1.5 \mathrm{~kg} / \mathrm{d}-\mathrm{ha}$ and retention time of at least 15 days is needed to achieve only a $1.5 \mathrm{mg} / \mathrm{L}$ reduction in total phosphorous (U.S. EPA, 1999). Phosphorus removal in the literature averages in $0.12 \mathrm{~g} \mathrm{P} / \mathrm{m}^{2}$-day, considered a low removal rate (Vymazal, 2005). 
System maturity, climate, maintenance, flow fluctuation, type of plants, and system configuration affect constructed wetlands performance. The average level of function in mature constructed wetlands is generally less than that for natural wetlands. Rate and time of maturation and functional level at maturity will differ from project to project, depending on the type of wetland being restored/constructed. Changes in the characteristics of constructed wetlands can be expected in response to the maturation process, but also in response to changes in the environment. Over time, successful constructed wetlands can be expected to become similar to comparable natural wetlands. Suspended solids are removed primarily by settling in the flow management basin; however, filtering within the constructed wetlands system increases as the plant density increases with system maturity. Vegetation density at full maturity will influence the wetlands' ability to promote sedimentation of TSS and associated BOD (Rodgers, et al., 2002).

CWs are similar to "natural" systems. As a result, operation is mostly passive and requires little operator intervention. Operation involves simple procedures similar to the requirements for operation of a facultative lagoon. The operator must be attentive, take appropriate actions when problems develop, and conduct required monitoring and operational monitoring as necessary. Clogging is considered one of the worst problems during CW's operations as it involves chemical, physical and biological processes (Knowles, et al., 2011) (Wallace \& Knight, 2006) (Cooper, et al., 2005). Clogging reduces the life of the $\mathrm{CW}$ system and may reduce the system efficiency as well (Caselles-Osorio, et al., 2007) (Rousseau, et al., 2005). 
The most critical monitoring issue during the $\mathrm{CW}$ startup period is vegetation growth and coverage. The planting strategy, combined with hydro period control as the plants grow, determines the effectiveness of vegetation growth during the startup period. Once the wetland vegetation has been established, the system can be brought online and the wastewater introduced. Most important in the operation of a SF constructed wetland is monitoring hydraulic and organic loadings to, and discharge(s) from, the wetland system (including the monitoring of individual wetland cells). Such tasks require measuring influent and effluent flow rates, and recording water depths in each wetland cell (U.S. Army Corps of Engineers, 2003).

Constructed wetlands require very low maintenance. The maintenance plan depends on the complexity of the constructed wetland design. Simple structures may only require mowing, banks and inlet inspection, outlet structures for signs of clogging, replacing damaged plants, pulling out undesirable plant species and sediment removal routines. More complex structures with mechanical devices such as valves or pumps may require much more detail, including maintenance recommendations from the manufacturer. Establishing preventive and corrective maintenance plan is recommended (Schueler, 1992). If a maintenance plan is not followed, constructed wetlands lifetime may be shortened due to several aspects, such as, spread of undesired plant species, clogging pipes, a broken pump and so forth. Weed invasion can dramatically reduce the ability of wetlands to meet its design objectives. They can form dense mats, exclude light and reduce dissolved oxygen in the water column, and increase the movement of nutrients through the system. Water level management is crucial to control weed growth. 


\subsubsection{Constructed Wetland Design}

Determining the wastewater flow is the most important feature for the design of any wastewater treatment plant. The U.S. EPA gives a range of 47-52 gallons per person per day (U.S. EPA, 1980). These values may vary according to the location and country. Average flows per home can be developed, which may range from 115 to 222 gallons per home per day. Infiltration and precipitation should also be considered in the wastewater flow. Infiltration and inflow (I/I) can be both seasonal and unpredictable. Annual records of seasonal flows are essential to determine the ultimate hydraulic capacity of the wastewater treatment facility.

The designer needs to know the expected BOD, TSS, total nitrogen, and total phosphorus when designing a constructed wetland. Table 1-1 lists the typical domestic raw wastewater concentration values.

Table 1-1: Typical domestic raw wastewater concentration, mg/L

\begin{tabular}{|l|r|c|}
\hline \multicolumn{1}{|c|}{ Parameter } & $\begin{array}{c}\text { Raw Wastewater } \\
\text { Concentration, range (typical) } \\
\text { (Burks \& Minnis, 1994) }\end{array}$ & $\begin{array}{c}\text { Facultative Lagoon Effluent } \\
\text { Concentration, range } \\
\text { (Reed, et al., 1995) }\end{array}$ \\
\hline BOD, mg/L & $100-400(250)$ & $30-40$ \\
\hline TSS, mg/L & $100-400(220)$ & $40-100$ \\
\hline Total Nitrogen, $\mathrm{mg} / \mathrm{L}$ & $15-90(40)$ & - \\
\hline Total Phosphorus, mg/L & $5-20(12)$ & - \\
\hline \begin{tabular}{|l|l|} 
Fecal Coliform, colonies/100 \\
mL
\end{tabular} & $10^{6}-10^{8}\left(10^{7}\right)$ & - \\
\hline Oil \& Grease, mg/L & & \\
\hline
\end{tabular}

All wastewater treatment facilities are ultimately affected by the water temperature.

Constructed wetlands are very temperature dependent as well. It is important to obtain 
information such as, monthly average temperatures, precipitation, evapotranspiration, soil temperatures at 30 inches and solar radiation. In absence of climatic records, examination of local vegetation can be correlated to plant hardiness zones and subsequently to probable minimum temperatures.

Evapotranspiration (ET) can be a major factor in the effectiveness of a constructed wetland. Increasing ET will result in higher hydraulic retention times since the outflow rate decreases. This could result in lower effluent BOD and TSS values. However the evaporation will also concentrate conservative, non-degradable contaminants such as phosphorous, increasing their concentrations in the effluent. Under extremely high temperature and low humidity levels, the amount of ET could approach or exceed the inflow rate into the system resulting in reduced water depth, which could damage some of the vegetation in the system. The use of a submerged bed system minimizes the effects of ET.

\subsubsection{Common Constructed Wetland Design Models}

Models are commonly found for the design of constructed wetlands in temperate climate areas (mainly the U.S.) to determine the size of a constructed wetland system. Among others, the most known are those by Campbell \& Ogden (1999), Reed et al. (1995), Environmental Protection Agency (U.S. EPA, 1999) and Kadlec and Knight (1995). The Campbell \& Ogden model has equations to calculate the area necessary for removal of Biological Oxygen Demand (BOD), Total Suspended Solids (TSS), Ammonia and Nitrate. The model accounts for temperature effect via an Arrhenius-type relationship for the rate constant. The model by Reed et al. (1995) estimates the area necessary for 
removal of BOD and phosphorus. The authors state that the performance of constructed wetland systems can be described by first-order kinetics in a plug flow regime. EPA has also equations to calculate the area necessary for removal of BOD and TSS. Those equations are conservative, which results in larger wetland areas; the equations are only based on loading rates, and temperature is not accounted for. Kadlec and Knight (1996) model presents other equations to calculate the area necessary for removal of BOD, TSS, phosphorus and nitrogen. Kadlec's equation also follows a first-order kinetic rate expression in a plug-flow setting; this expression is a function of background concentration. First-order models are commonly used and known to be inadequate, but still the most appropriate model available to date (Vymazal \& Kropfelova, 2009). These common models are further described below:

\section{a) Campbell \& Ogden}

Campbell \& Ogden (1999) method has equations to calculate the area necessary for removal of BOD, TSS, Ammonia and Nitrate through SSF CW and SF CW.

Temperature is a very important factor in this method. Design equations for temperature dependent rate constant, area, hydraulic detention time are presented as follows.

\section{Design Equation for SSF CW:}

The BOD removal is estimated using the following equation:

$$
A s=\frac{Q^{*}(\ln C o-\ln C e)}{K_{t}{ }^{*}{ }^{*} n}
$$

Where: $\quad Q=$ flow, in $\mathrm{m}^{3} /$ day 


$$
\begin{aligned}
& \mathrm{Co}=\text { influent BOD }(\mathrm{mg} / \mathrm{L}) \\
& \mathrm{Ce}=\text { effluent BOD }(\mathrm{mg} / \mathrm{L}) \\
& \mathrm{K}_{\mathrm{t}}=\text { temperature-dependent rate constant } \\
& \mathrm{d}=\text { depth of gravel bed } \\
& \mathrm{n}=\text { porosity of gravel }
\end{aligned}
$$

The temperature-dependent rate constant for SSF CWs, $\mathrm{K}_{\mathrm{t}}$, requires the following adjustment, considering $\mathrm{T}$ as the water temperature in ${ }^{\circ} \mathrm{C}$ :

$K_{t}=1.104 *(1.06)^{(T-20)}$

The main factor considered on TSS removal is the wastewater velocity, because this process consists of filtration and hydraulic detention time. The equation for TSS removal is:

$$
T S S_{\text {eff }}=T S S_{\mathrm{inf}} *(0.1058+0.0011 * H L R)
$$

Where: $\quad$ HLR $=$ hydraulic loading rate $(\mathrm{cm} /$ day $)$

$$
\begin{aligned}
& \mathrm{TSS}_{\text {eff }}=\text { effluent } \mathrm{TSS} \text { in } \mathrm{mg} / \mathrm{L} \\
& \mathrm{TSS}_{\text {inf }}=\text { influent } \mathrm{TSS} \text { in } \mathrm{mg} / \mathrm{L}
\end{aligned}
$$

Nitrogen removal is a temperature-dependent process as the BOD removal process is, and the nitrogen in the form of nitrates is easier to be removed by wetlands than in the form 
of ammonia. The following formula calculates nitrogen removal, in the form of nitrates $\left(\mathrm{NO}_{3}\right)$ :

$\ln \left(N O_{3 \text { inf }} / N O_{3 e f f}\right)=K_{t} * H R T$ or $\quad H R T=\left[\ln \left(N O_{3 \text { inf }} / N O_{3 e f f}\right)\right] / K_{t}$

Where: $\quad \mathrm{K}_{\mathrm{t}}=1.15^{(\mathrm{T}-20)}$

$$
\begin{aligned}
& \mathrm{NO}_{3} \text { inf }=\text { influent nitrate in } \mathrm{mg} / \mathrm{L} \\
& \mathrm{NO}_{3} \text { eff }=\text { effluent nitrate in } \mathrm{mg} / \mathrm{L}
\end{aligned}
$$

Design Equation for SF CW:

The same formula used for SSF CW to calculate the area for BOD removal can be used for SF CWs, using a different reaction rate constant.

$$
A s=\frac{Q^{*}(\ln C o-\ln C e)}{K_{t}^{*} d^{*} n}
$$

Where: $\quad Q=$ flow, in $\mathrm{m}^{3} /$ day

$$
\begin{aligned}
& \mathrm{Co}=\text { influent BOD }(\mathrm{mg} / \mathrm{L}) \\
& \mathrm{Ce}=\text { effluent BOD }(\mathrm{mg} / \mathrm{L}) \\
& \mathrm{K}_{\mathrm{t}}=\text { temperature-dependent rate constant } \\
& \mathrm{d}=\text { water depth } \\
& \mathrm{n}=\text { Porosity (density of the plant stems) }
\end{aligned}
$$


The temperature-dependent rate constant for SF CWs, $\mathrm{K}_{\mathrm{t}}$, requires the following adjustment, considering $\mathrm{T}$ as the water temperature in ${ }^{\circ} \mathrm{C}$ :

$K_{t}=.278 *(1.06)^{(T-20)}$

TSS removal equation for a SF CW is presented below:

$T S S_{\text {eff }}=T S S_{\mathrm{inf}} *[.1139+.002(H L R)]$

The nitrogen removal equations are similar to the SSF CW, with different reaction rate constant for Ammonia removal. The reaction rate constants for nitrogen removal in a SSF CW are:

AmmoniaK $_{t}=.2187 *(1.048)^{(T-20)}$

Nitrate $_{t}=1,15^{(T-20)}$

b) Reed et al.

Reed et al. method has equations to calculate the area necessary for removal of BOD and phosphorus through SSF CW and removal of BOD through SF CW. Reeds et al. states that all CW systems have their performances described with first-order plug-flow kinetics as shown below.

BOD Removal in SF CW:

The design surface area of the wetland is given by: 
$A=\frac{Q\left(\ln C_{0}-\ln C_{e}\right)-0.6539}{65 K_{T} d}$ (For slope smaller than 1 percent)

Where: $\quad t=$ hydraulic residence time in the system, days

$\mathrm{C}_{0}=$ influent $\mathrm{BOD}$ concentration, $\mathrm{mg} / \mathrm{L}$

$\mathrm{C}_{\mathrm{e}}=$ effluent $\mathrm{BOD}$ concentration, $\mathrm{mg} / \mathrm{L}$

$\mathrm{K}_{\mathrm{T}}=$ reaction rate constant, days ${ }^{-1}=K_{T}=K_{20}(1.1)^{(T-20)}$

$\mathrm{K}_{20}=$ rate constant at $20^{\circ} \mathrm{C}=0.0057$ days $^{-1}$

$\mathrm{d}=$ design water depth in the system

$\mathrm{T}=$ temperature,${ }^{\circ} \mathrm{C}$

Hydraulic residence time is given by:

$t=\frac{\left(\ln C_{0}-C_{e}\right)-0.6539}{65 K_{T}} \quad$ (For slope smaller than 1 percent)

Other values recommended:

Organic loading $<112 \mathrm{~kg} \mathrm{BOD/(ha} \cdot$ day) $[100 \mathrm{lb} /($ acre $\cdot$ day $)]$

Specific surface are for attached microbial growth $=15.7 \mathrm{~m}^{2} / \mathrm{m}^{3}$

Porosity (n value) of wetland flow path $=0.75$

Aspect ratio $(\mathrm{L} / \mathrm{W})>10: 1$

Water depth, warm months $<10 \mathrm{~cm}$, cool months $<45 \mathrm{~cm}$. 
BOD Removal in SSF CW:

$$
\frac{C_{e}}{C_{o}}=\exp \left(-\frac{K_{T} A_{S} d n}{Q}\right)
$$

Where: $\quad \mathrm{C}_{\mathrm{e}}=$ effluent $\mathrm{BOD}, \mathrm{mg} / \mathrm{L}$

$$
\begin{aligned}
& \mathrm{C}_{\mathrm{o}}=\text { influent } \mathrm{BOD}, \mathrm{mg} / \mathrm{L} \\
& \mathrm{K}_{\mathrm{T}}=\text { first-order temperature-dependent rate constant, days }{ }^{-1} \\
& \mathrm{~A}_{\mathrm{S}}=\text { surface area of the system, } \mathrm{m}^{2}\left(\mathrm{ft}^{2}\right) \\
& \mathrm{n}=\text { bed porosity (as a decimal fraction) } \\
& \mathrm{d}=\text { depth of submergence, } \mathrm{m}(\mathrm{ft}) \\
& \mathrm{Q}=\text { average flow through system, } \mathrm{m}^{3} / \text { day }\left(\mathrm{ft}^{3} / \text { day }\right) \\
& K_{T}=K_{20}(1.1)^{(T-20)}
\end{aligned}
$$

Where: $\quad \mathrm{K}_{20}=$ rate constant at $20^{\circ} \mathrm{C}$

$$
\begin{aligned}
& \mathrm{T}=\text { operational temperature of system, }{ }^{\circ} \mathrm{C} \\
& \mathrm{K}_{\mathrm{T}}=\text { first-order, temperature-dependent rate constant, days }{ }^{-1}
\end{aligned}
$$

It is possible to determine the surface area required for a SSF CW by taking the natural logarithm of both sides of the equation above and rearranging it.

$$
A=\frac{Q\left(\ln C_{0}-\ln C_{e}\right)}{K_{T} d n}
$$


Where:

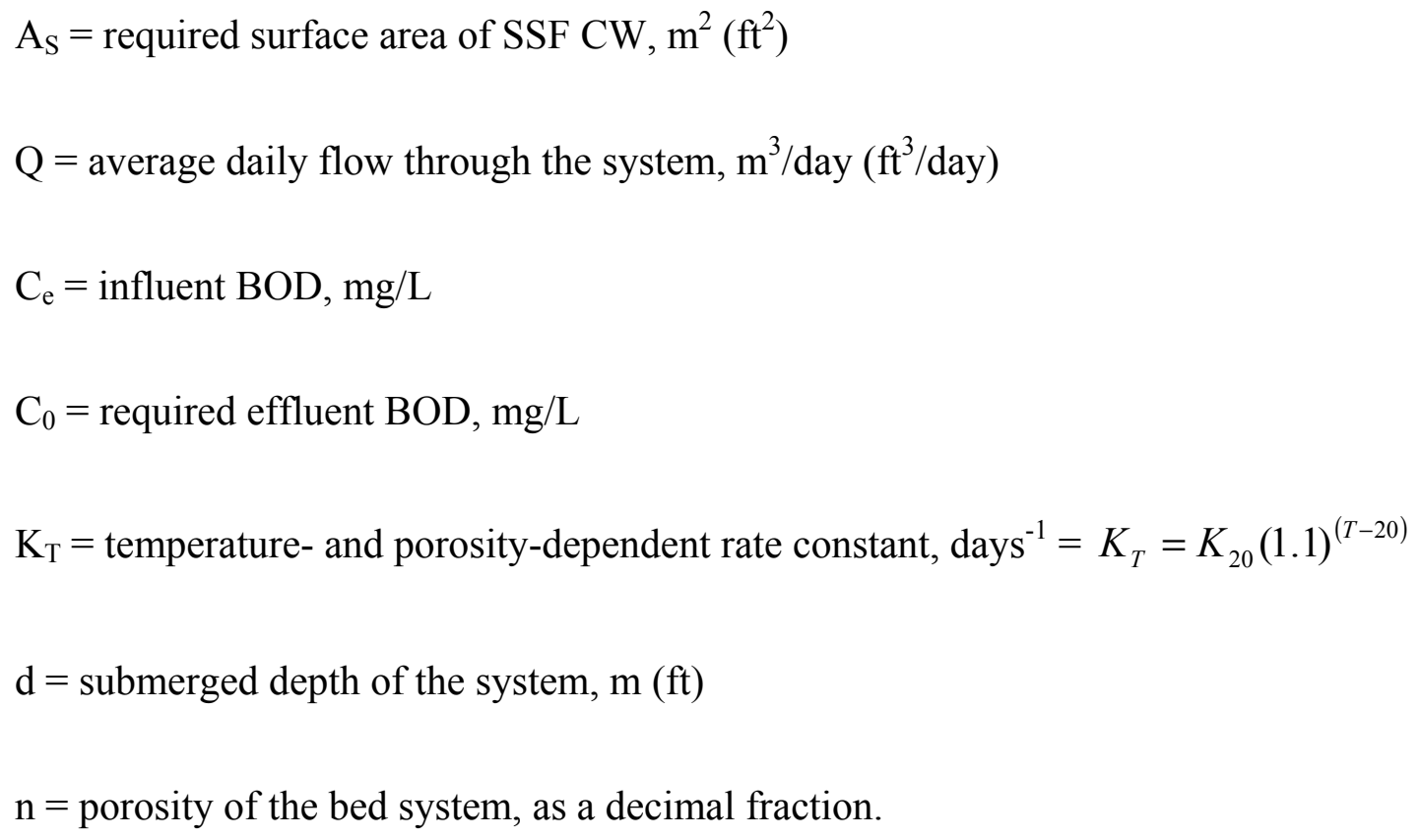

c) $\underline{\text { EPA }}$

EPA method has equations to calculate the area necessary for removal of BOD and TSS through SSF CW and removal of BOD, TSS, Kjeldahl and ammonia through SF CW. EPA suggests a very conservative method of calculation which yielded very large wetland areas. EPA equations are based on loading rates only, and temperature is not a differential factor.

$$
A L R=Q C_{o} / A_{W}
$$

Where: $\quad A L R=$ areal loading rates: $B O D=40 \mathrm{~kg} / \mathrm{ha}-\mathrm{d} ; \mathrm{TSS}=30 \mathrm{~kg} / \mathrm{ha}-\mathrm{d}$

$$
\mathrm{Q}_{0}=\text { incoming flow rate, in } \mathrm{m}^{3} / \mathrm{d}
$$




$$
\begin{aligned}
& \mathrm{C}_{\mathrm{o}}=\text { influent concentration, in } \mathrm{mg} / \mathrm{L} \\
& \mathrm{A}_{\mathrm{W}}=\text { total area of SF CW, in ha }
\end{aligned}
$$

\section{d) Kadlec}

Kadlec method has equations to calculate the area necessary for removal of BOD, TSS, phosphorus and nitrogen through SSF CW and SF CW. Kadlec calculates area based on background concentration and reaction rate constant derived from multiple operational wetlands. Background concentration $\left(\mathrm{C}^{*}\right)$ is defined as the concentration that cannot be removed from the wastewater, with equations to estimate their $\mathrm{C}^{*}$ for TSS and $\mathrm{BOD}_{5}$, as presented below. The general equation for Kadlec's method is shown below.

$\ln \left(\frac{C_{e}-C^{*}}{C_{i}-C^{*}}\right)=-\frac{k}{q}$

Where: $\quad \mathrm{C}_{\mathrm{e}}=$ outlet target concentration, $\mathrm{mg} / \mathrm{L}$

$$
\begin{aligned}
& \mathrm{C}_{\mathrm{i}}=\text { inlet concentration, } \mathrm{mg} / \mathrm{L} \\
& \mathrm{C}^{*}=\text { background concentration, } \mathrm{mg} / \mathrm{L} \\
& \mathrm{C}^{*}{ }_{\text {BOD }}=3.5+0.053 \mathrm{Ci}(\text { For SSF and } \mathrm{SF} \text { ) } \\
& \mathrm{C}^{*} \text { TSS }=7.8+0.063 \mathrm{Ci} \text { (For SSF) } \\
& \mathrm{C}^{*}{ }_{\text {TSS }}=5.1+0.16 \mathrm{Ci}(\text { For } \mathrm{SF}) \\
& \mathrm{k}=\text { first-order areal rate constant, } \mathrm{m} / \mathrm{yr} \\
& \mathrm{q}=\text { hydraulic loading rate, } \mathrm{m} / \mathrm{yr}
\end{aligned}
$$


The area required for a particular pollutant is given by the following equation:

$$
A=\left(\frac{0.0365^{*} Q}{k}\right) * \ln \left(\frac{C_{i}-C^{*}}{C_{e}-C^{*}}\right)
$$

Where: $\quad \mathrm{A}=$ required wetland area, ha

$$
\mathrm{Q}=\text { water flow rate, } \mathrm{m}^{3} / \mathrm{d}
$$

The concentration of all pollutants is computed with the following equation, using the largest area:

$$
C_{o}=C^{*}+\left(C_{i}-C^{*}\right) \exp \left(-\frac{k A}{0.0365 Q}\right)
$$

Where: $\quad \mathrm{C}_{\mathrm{o}}=$ outlet concentration, $\mathrm{mg} / \mathrm{L}$

e) Other Models

There are other models to calculate the size of the $\mathrm{CW}$ area that require many parameters that are usually hard to measure (Sklarz, et al., 2010) (Marsili-Libelli \& Checchi, 2005) (Rousseau, et al., 2004) (Mashauri \& Kayombo, 2002), including:

- Monod kinetics: interrelation on substrate availability and growth of biomass for predicting nitrogen and organics dynamics in wetland systems with continuousstirred tank reactor or plug flow pattern to predict effluent concentrations (Saeed \& Sun, 2011); 
- Multi-flow dispersion model: the total flow is divided into a number of different flow fractions that follows several pathways each with unique hydraulic features, applied to the plug-flow with dispersion model to obtain different dispersion modules and average hydraulic residence time values (Maloszewski, et al., 2006).

These other models were not further reviewed in this study.

\subsubsection{Role of Plants in Constructed Wetlands}

The role of plants in CWs is debatable (Mara, 2004), with most studies indicating the selection of plants to use in constructed wetlands as an important aspect of the design (Vymazal \& Kropfelova, 2009); and depending on the type of plant selected, pollutant removal may be improved (Akratos \& Tsihrintzis, 2007) (Brisson \& Chazarenc, 2009) (Kadlec, et al., 2000) (Stefanakis \& Tsihrintzis, 2012). Other studies indicate that similar treatment efficiencies through CWs can be achieved independently of the presence or absence of plants, especially for ammonium removal (Sklarz, et al., 2009) (Gross, et al., 2007) (Lahav, et al., 2001) (Green, et al., 1998).

The presence of wetland plants has many benefits to the surface flow wetland. They provide surface area for attached bacterial growth, they provide shade that reduces the growth of algae which can cause suspended solids problems in the effluent, reduce wind turbulence which could cause resuspension of settled solids, transfer limited amounts of oxygen into subsurface soils of rock media in a submerged bed system. On the other hand, dense planted areas interfere with oxygen transfer into the waste and prevent 
sunlight penetration, which can stimulate photo destruction of certain contaminants and oxygen production by algae. Open water parts of the wetland or parts containing mostly submerged growth may exhibit aerobic conditions, at least throughout the day. Heavily vegetated parts probably exhibit anoxic or anaerobic conditions throughout. Plants in a subsurface flow wetland have been not found to be as beneficial as in a surface flow wetland. Their major contribution is to limit the access of people and animals to the top of the gravel bed, which may be wet with partially treated wastewater. They also provide aesthetic appeal and ecological benefits since many of the plants that can thrive in such an environment are attractive and provide habitat to wildlife. Some plants have the potential to transfer oxygen down into the gravel bed and transport waste product gases, such as methane, $\mathrm{CO}_{2}$ and nitrogen out of the bed. However, studies indicate that the rate of gas transfer is small compared to the oxygen demand of the wastewater and thus has little impact on the biodegradation reactions occurring within.

The type of vegetation depends on the goals and objectives of the wetland. Ideally, vegetation should include a variety of species; however, constructed wetlands for treating wastewater need to be as versatile and easily maintained as possible. Practicality might dictate limiting plant species to the hardiest, commonly found, and easily managed. Plant species' performance and removal efficiency is tied to the biota's ability to tolerate the extremely variable conditions. Emergent and floating aquatic plants provide a shade over the water column, limiting phytoplankton production, increasing the potential for accumulation of free-floating aquatic plants that restrict atmospheric re-aeration and the reducing suspended solids within the constructed wetland (U.S. EPA, 1999). Some commonly used plants are shown on the Table 1-2. 
There is literature available about characteristics and requirements of various wetland plants. Demonstrational research has found that cattails (Typha spp.) and reed canary grass (Phalaris arundinacea) have proven to be low cost, easy to establish, low maintenance, and tolerant of a wide range of climatic and contamination conditions. Cattails and reed canary grass can both tolerate drought conditions for several weeks. Broadleaf cattails (Typha latifolia) can withstand water depths up to 18 inches and narrowleaf cattails (Typha angustifolia) up to 12 inches. The next most versatile and easily managed plants would be common reed (Phragmites australis) and various species of bulrush (Scirpus spp.).

Table 1-2: Common plants for constructed wetlands

\begin{tabular}{|l|l|}
\hline \multicolumn{1}{|c|}{ Type of plants } & \multicolumn{1}{c|}{ Common Species } \\
\hline Free Floating Aquatic & Common duckweed (Lemna), Big duckweed (Spirodela). \\
\hline Rooted Floating Aquatic & Water lily (Nymphea), Pennywort (Hydrocotyle) \\
\hline Submerged Aquatic & Pondweed (Potamogeton), Water weed (Elodea) \\
\hline Emergent Aquatic & Cattail (Typha), Bulrush (Scirpus), Common Reed (Phragmites) \\
\hline Shrubs & Dogwood (Cornus), Holly (Ilex) \\
\hline Trees & Maple (Acer), Willow (Salix) \\
\hline Source: (U.S. EPA, 1999) & \\
\hline
\end{tabular}

\subsection{Research Gaps}

There are studies and experiences of constructed wetlands in the temperate climate (mostly in the U.S. and Europe); in contrast, information is limited in the tropics (Caselles-Osorio, et al., 2011) (Mburu, et al., 2013). The effectiveness of wetland 
systems is affected by climate; important factors include temperature, precipitation and evapotranspiration, which vary from place to place. As a result, experiences and models from temperate climate may not transfer well to the tropics due to the difference in climate. It is generally assumed that constructed wetland systems are more efficient in tropical climates than in temperate climates because higher temperatures encourage plant growth and enhances the kinetics of microbial activity (Bojcevska \& Tonderski, 2007); (Kaseva, 2004); (Trang, et al., 2010).

However, there is very limited number of $\mathrm{CW}$ studies that use commonly found tropical plants, such as Thalia, Cyperus, and Brachiaria.

Additionally, most studies investigate the $\mathrm{CW}$ performance for BOD, and nutrients (total nitrogen and phosphorus); however, not many studies present $\mathrm{CW}$ performance results for fecal coliforms, TSS, and Oil \& Grease. Fecal coliforms is a parameter of interest under tropical climate conditions as higher temperatures, precipitation and humidity are prone to their proliferation.

\subsection{Central Hypothesis and Research Objectives}

The central hypothesis is that the constructed wetland system of the study is capable to provide competitive secondary treatment levels, removing significant amounts of biological oxygen demand $\left(\mathrm{BOD}_{5}\right)$, total suspended solids (TSS) and pathogens among other water quality indicators, under tropical climatic conditions.

The research objectives are as follows:

1) Design, construction, startup, and operation phases of the field scale CW System. 
2) Characterize SSF and SF influent and effluents for BOD, TSS, Total Nitrogen, Phosphorus, Fecal Coliform, and Oil \& Grease.

3) Evaluate the design performance using empirical models and observed data.

4) Compare the differences in treatment associated with wetland plants commonly used in CW and plants that are typical to the tropics, not traditionally used in CWs.

\subsection{Justification and Contribution}

The U.S. Department of Defense, in conjunction with Florida International University (FIU), conducted a demonstration and validation of innovative technologies in order to allow an efficient management of military installations, protect the environment, ensure safety and occupational health, and develop renewable clean energy resources.

Constructed wastewater treatment wetland systems in a tropical climate were one of these innovative technologies selected to reduce the discharge of untreated or partially treated wastewater into surface water streams. Such discharges are a risk to human health and cause environmental degradation.

The constructed wetlands demonstration and validation evaluated in this dissertation took place in El Salvador, Central America and focused on determining the effectiveness of treatment wetlands as compared to conventional wastewater treatment methods. The overall purpose of this project was to plan, construct and evaluate a constructed treatment wetland to gain mutually beneficial experience with such system in tropical climate. This 
study monitored and evaluated the complexity of the design process, evaluated the suitability of this technology in tropical climate, and assessed the use of typical tropical plants in constructed wetland performance efficiency. Moreover, the predicted values used during design were compared with actual results from the constructed system to evaluate the design methodology appropriateness.

\subsection{Dissertation Outline}

This dissertation is organized in four chapters. The first chapter is an introduction including background information on constructed wetland systems, research objectives, current research gaps, and a brief description on how this project was initiated by the U.S. Department of Defense and FIU.

Chapter two consists of the experimental setting, including the study location, system description, an overview of the constructed wetland system design and construction, and presents the data collected in this study, such as water balance, quality indicators, and field parameters.

Chapter three includes the results and discussion addressing the objectives of this dissertation. The CW System of this study performance results are presented for $\mathrm{BOD}_{5}$, TSS, COD, Fecal Coliform, Oil \& Grease, Total Nitrogen, Phosphorus, Plant Growth and Health) and evaluated in comparison with the available literature information. An assessment of the models used during design typically used in temperate climate is compared with the actual performance results from this study $\mathrm{CW}$ system which is in a 
tropical climate. Sensitivity analysis of the methodology used during design was performed for $\mathrm{BOD}_{5}$ and TSS to determine the variables that have an important role in the CW system process.

Chapter four presents the conclusions, limitations and recommendations as reference for future investigations and applications. 


\section{METHODOLOGY}

\subsection{Experimental Setting}

\subsubsection{Study Location}

The field-scale constructed wetland system of this investigation was part of a demonstration project that was conducted by Florida International University (FIU), in conjunction with the United States and the El Salvadoran Armies. The project treated domestic wastewater generated from approximately 600 soldiers and was located at the Salvadoran Army Cavalry Regiment (SACR), near San Juan Opico, La Libertad Province, in El Salvador, as shown in Figure 2-1.

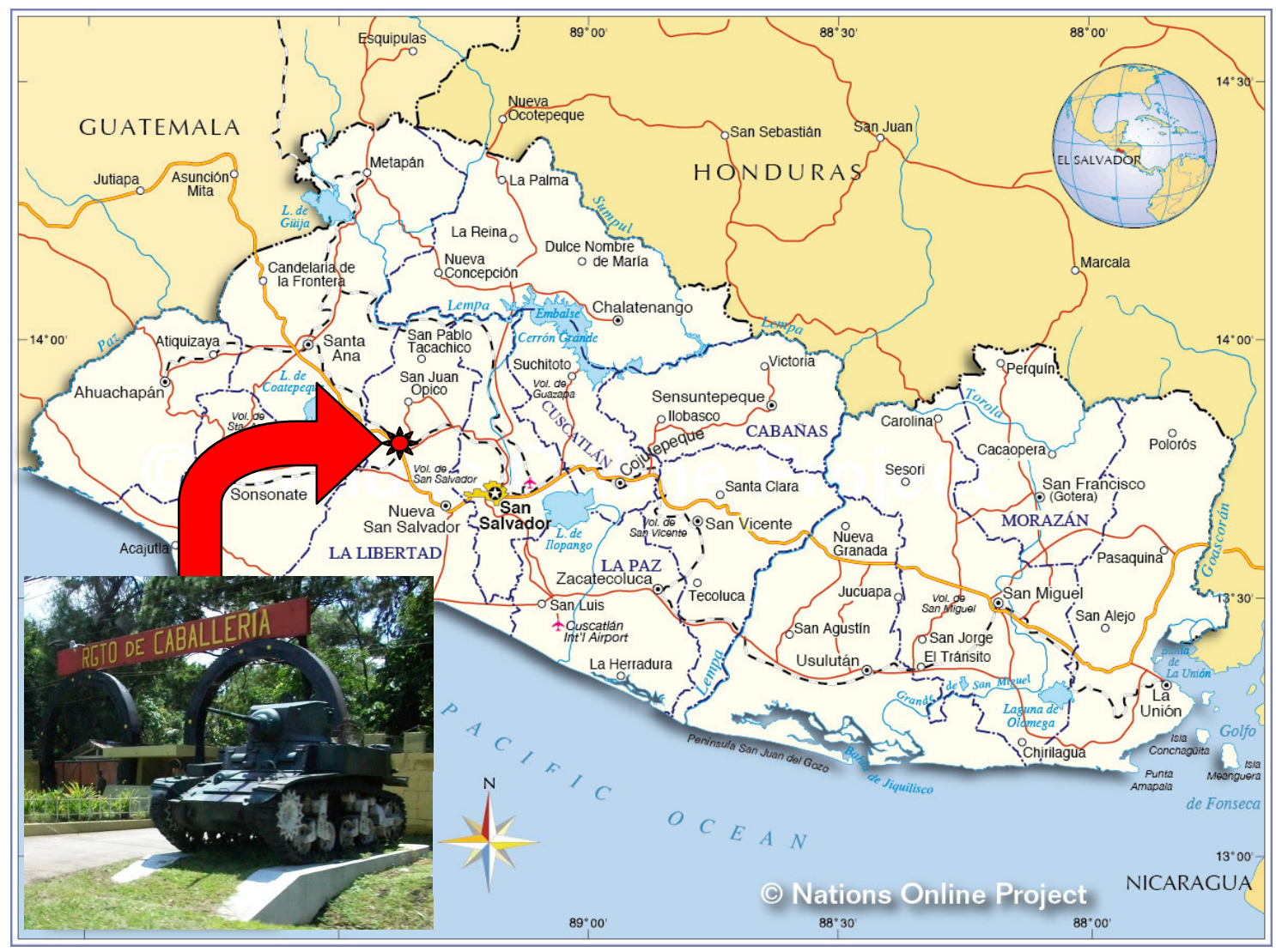

Figure 2-1: Location Map 
The site is at an altitude of $460 \mathrm{~m}$ above sea level and is of coordinates $13^{\circ} 48.5^{\prime} \mathrm{N}$ and $89^{\circ} 24.4^{\prime} \mathrm{W}$. The region is characterized by a hot tropical climate and a savanna-like woodland ecosystem. Two seasons, alternating with two 30-day transitional periods occur during the year: the dry season falls between November and April, and the wet season lasts from May to October. Every year the area receives an average of $1644 \mathrm{~mm}$ of rainfall, with monthly maximums approaching $317 \mathrm{~mm}$ during the rainy season. Daytime temperatures vary little, ranging from January lows of $22.7^{\circ} \mathrm{C}\left(72.9^{\circ} \mathrm{F}\right)$ to highs of $26.0^{\circ} \mathrm{C}$ $\left(78.8^{\circ} \mathrm{F}\right)$ during April-May (Katsenovich, et al., 2009).

\subsubsection{System Description}

Prior to the construction of the constructed wetland system in 2005, wastewater at the SACR received only partial treatment in the facultative lagoon (FL) system, followed by the addition of chlorine solution for disinfection, and then the partially treated effluent was discharged into an outfall ditch, as illustrated in Figure 2-2.

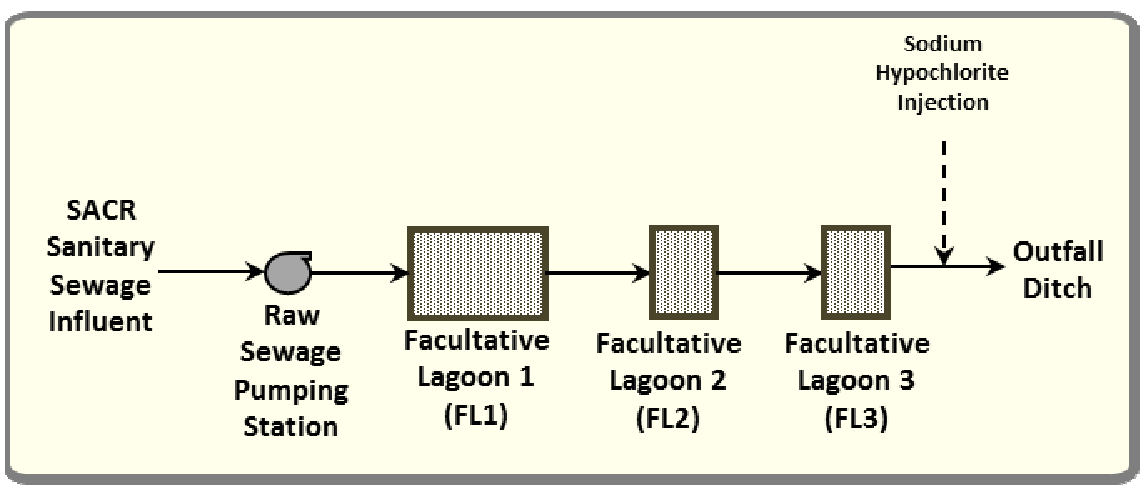

Figure 2-2: Facultative Lagoon System Schematic (Prior to CW System Construction) 
Figure 2-3 shows a schematic of the complete new treatment system that includes the constructed wetland system (CW system) of this study. The new CW system is in between the three existing facultative lagoons that are now called Basins 1 to 3. Figure 2-4 shows the site layout for the complete treatment system. As mentioned above, this dissertation focuses on the CW system, consisting of three SSF CWs and three SF CWs. An aerial view of the complete system is included in Appendix A.

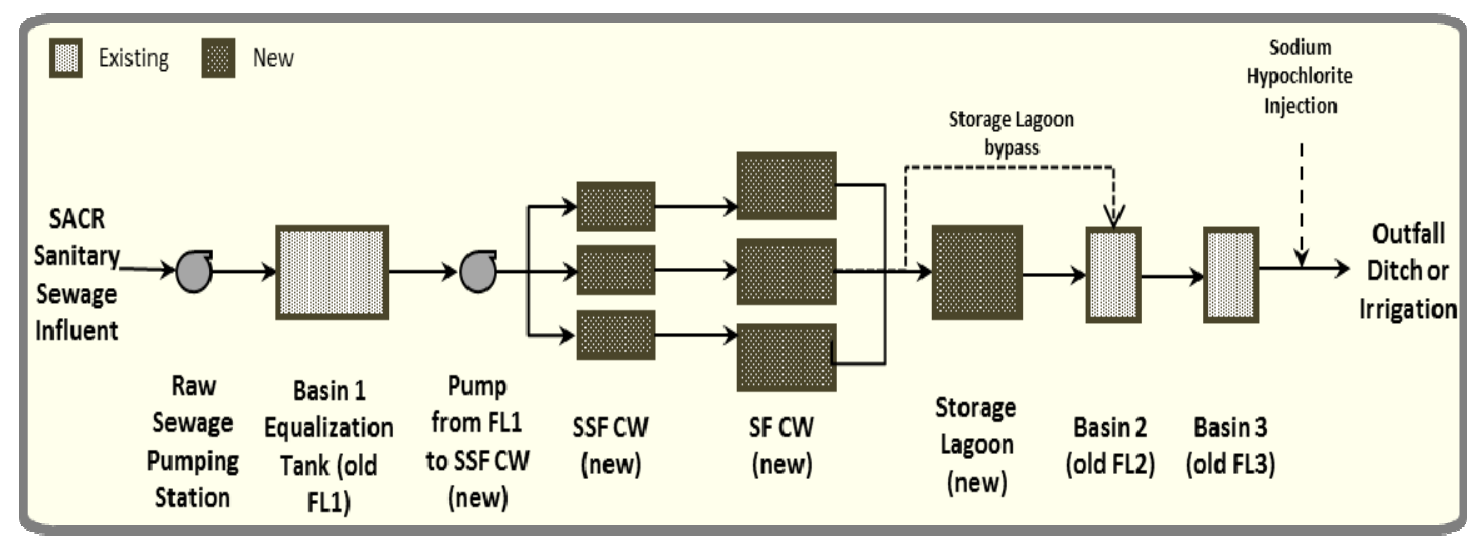

Figure 2-3: Complete Treatment System Schematic (new) 


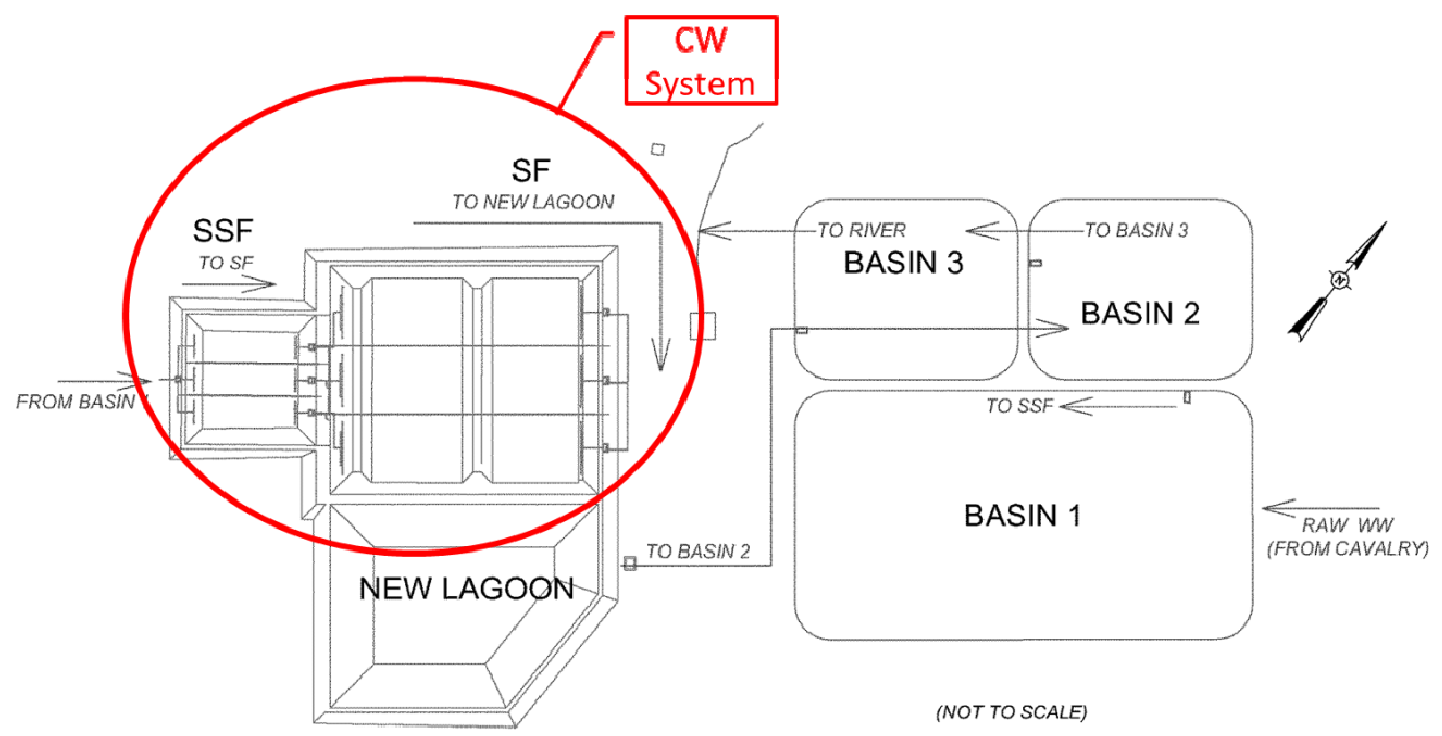

Figure 2-4: Site Plan with Constructed Wetland System

The CW system was placed near an existing facultative lagoon system at the Cavalry Base (now called Basins 1, 2 and 3). Basin 1, which became an equalization basin, providing some partial primary treatment, has a capacity of $9,800 \mathrm{~m}^{3}$ and it is followed by the SSF CW and the latter followed by a SF CW; the SF CW effluent was then discharged in a new lagoon, then into Basins 2 and 3, depending on operational and possible reuse needs in irrigation.

A $150 \mathrm{~m}^{3} /$ day (40,000 gallon per day) pump was installed in the existing flow control structure connecting Basin 1 to the SSF CW. The pump was initially controlled by a simple float switch system and operated based on water level to pump the entire wastewater flow coming out of the first basin to the new wetland system. Since the float switch did not work properly for the entire duration of this study, a timer was installed to start and stop the pump on a regular basis. Water treated by the constructed wetland 
system flowed by gravity from the SSF CW to the SF CW and to the new lagoon and then to Basin 2 where it was further cleaned and stored for possible use in irrigation. If not used, the water passed through Basins 2 and 3 and exited through the existing wastewater discharge point.

The SSF CW unit consisted of a 0.6-meter thick bed of 20-30 mm diameter gravel (medium gravel). At the entrance and exit ends of each bed a zone of 40-80 mm diameter coarse gravel allowed for a uniform distribution of flow across the wetland gravel bed. A 0.15-meter thick layer of 5-10 $\mathrm{mm}$ diameter fine gravel was over both gravel zones to serve as rooting zone for the wetland plants. The gravel beds were separated into three parallel flow paths to improve the distribution of wastewater and reduce short-circuiting.

The SF CW unit consisted of a shallow open basin containing alternating regions of open water $(\mathrm{OW})$ with submerged aquatic plants and regions of shallower water filled with emergent wetland vegetation. The OW portion of these basins was approximately 1.2 meters deep where atmospheric oxygen, and oxygen generated by submerged plants and algae, interacts with bacteria to change the nature of contaminants in the water, particularly nutrients such as ammonia. The flow then passed into the vegetated sections where interactions between the plants and bacteria growing on the plants, removed these contaminants. In these areas wastewater flowed in an open channel approximately 0.45 meters deep. Like the SSF CW, this wetland bed was divided into three parallel flow paths. Flow from each of the SSF flow paths passed directly into one of the SF CW flow paths through a submerged inlet distribution header. Water flowed into the first open water section, a vegetated section, a second open water section, and then the final 
vegetated section. Treated water passed through a narrow gravel bed to remove floating solid matter released from the vegetated sections. Treated water then passed into an effluent collection header identical to the inlet distribution header. The water passed through a level control structure fitted with a swiveling overflow pipe that could be tilted to allow the water level to be adjusted as needed.

Plans and sections of both SSF and SF CW systems, including dimensions, are presented in Appendix A.

\subsubsection{Design and Construction}

\subsubsection{Area Calculation}

The design of the above mentioned CW System was completed in a step-wise iterative manner following the steps discussed in this Chapter, by former FIU engineer L. Moos, and myself. The basic process design was selected after reviewing a number of current design approaches in common use in the USA for this type of system. The design methodologies described in Chapter 1.1.1.1 and listed below were examined and compared for BOD and TSS removals:

- Campbell, C.S. and Michael H. Ogden. Constructed Wetlands in the Sustainable Landscape. John Wiley \& Sons, Inc. 1999.

- EPA. "Constructed Wetlands Treatment of Municipal Wastewaters." Office of Research and Development. EPA/625/R-99/010. September 2000. 
- Kadlec, R.H. and Robert L. Knight. Treatment Wetlands. CRC Lewis Publishers. 1995.

- Reed, S. C., E. Joe Middlebrooks and Ronald W. Crites. Natural Systems for Waste Management \& Treatment. McGraw-Hill Book Company. 1988.

The various design calculations were done in a series of Microsoft Excel worksheet, validated by hand calculations of each major calculation. The main input parameters for the various models were wastewater flow, influent characteristics and temperature. Several of the models introduced other parameters unique to their methodology. This analysis revealed a wide range in design approaches and in the resulting calculation of required wetland areas.

Flows, wastewater analysis, nearby water river analysis, well water analysis and precipitation, evapotranspiration and temperature information were the main parameters used on this design. These data were collected from two trips to the Army Bases in El Salvador in July and August 2005. Wastewater and water samples were collected by FIU personnel (L. Moos and myself), and sent to FUSADES Laboratory (a local laboratory) for necessary analysis. Precipitation, evapotranspiration and temperature were provided by CENTA ("Centro Nacional de Tecnologia Agropecuaria y Forestal").

The base flow for approximately 600 soldiers was measured as 22,400 gal/day, resulting in a daily wastewater flow of approximately $37 \mathrm{gal} /$ capita-day (140 L/capita-day). September was the peak flow month because of the large amount of precipitation with the estimated design flow rates as shown in Table 2-1, and therefore, it was used as the 
design month for comparison of required areas. Figure 2-5 illustrates the annual precipitation, evaporation and temperature used in the design process.

Table 2-1: Design Flow Rates Used in Method Comparison

\begin{tabular}{|l|c|}
\hline \multicolumn{1}{|c|}{ Location } & Flow (gal/day) (September) \\
\hline Base Flow (wastewater only)* & 22,400 \\
\hline Basin 1 outlet flow & 30,496 \\
\hline SSF CW outlet & 31,909 \\
\hline SF CW outlet & 38,974 \\
\hline$* 600$ soldiers, approximately 37 gal/capita-day (140 L/capita-day) \\
\hline
\end{tabular}

The design comparison was based on wastewater characteristics estimated for the month of September 2005 as explained above. BOD removal was the controlling process for the SSF CW sizing. A comparison of the areas calculated showed that the Kadlec method yields an area for the SSF CW comparable though somewhat smaller than the other models, and much smaller than the EPA method. The CW area was calculated for the projected influent flow rates and contaminant characteristics for each month of the year, based on the following assumptions:

- Temperature $=20.4$ degree $\mathrm{C}$

- $\quad$ SSF CW: Depth of gravel bed $=0.60 \mathrm{~m}(2$ feet $)$

Porosity of gravel $=0.40$

- $\quad \underline{\text { SF CW: }} \quad$ Depth of SF cell $=0.45 \mathrm{~m}$ (18 inches)

Porosity (density of plant stems) $=0.65$ 
Depth of OW cell $=1.20 \mathrm{~m}$ (4 feet)

Porosity (density of plant stems) $=0.80$

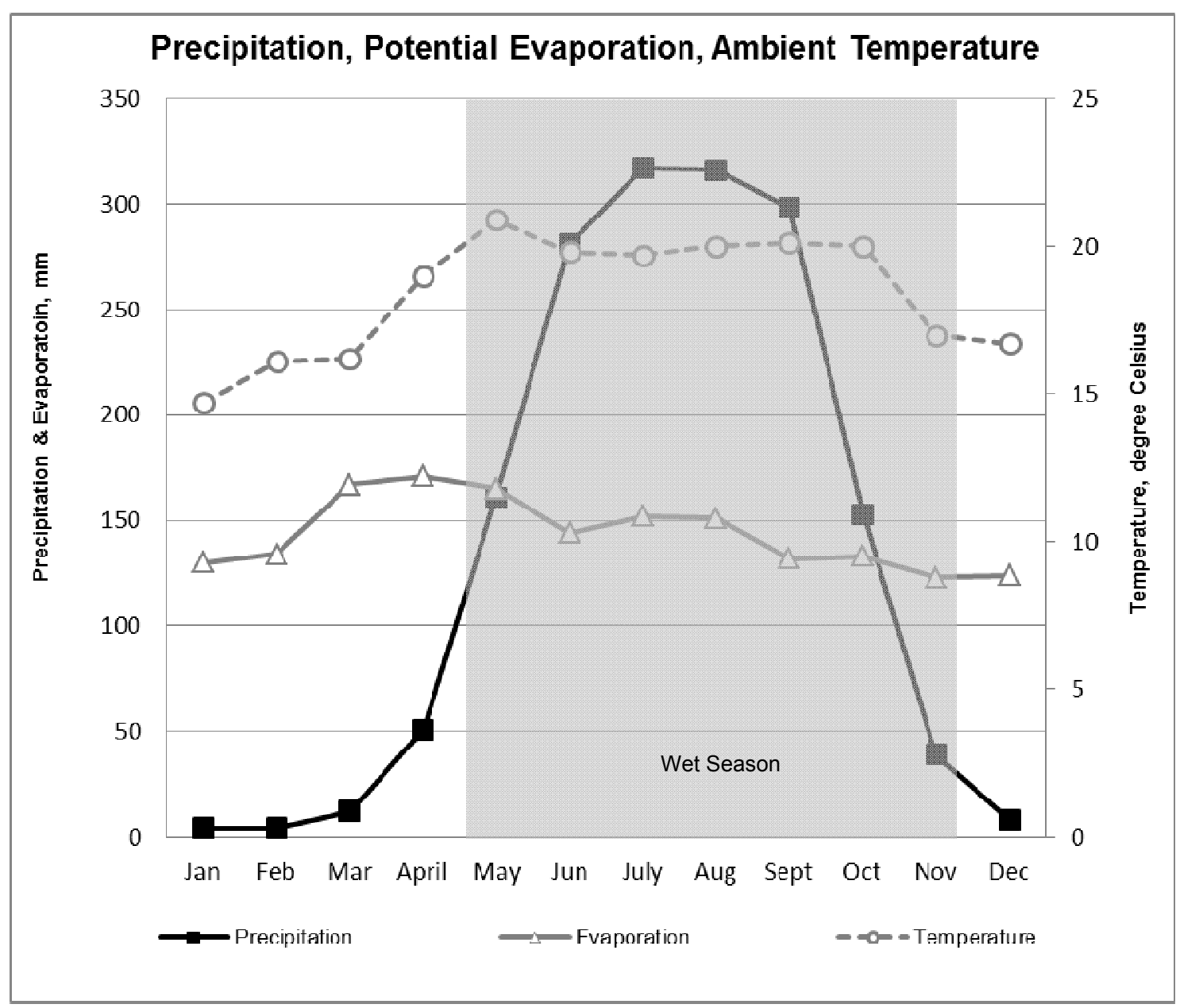

Figure 2-5: Precipitation, Evaporation, Temperature

Based on the calculation results of area and hydraulic detention time for each method mentioned above listed in Table 2-2, Kadlec methodology was chosen for final design.

Kadlec is the only method that provides calculation tools for all the parameters of interest in this study. As mentioned above, BOD removal was the decision parameter for the SSF CW dimensions, and Total Nitrogen removal was the decision parameter for the SF CW 
dimensions. The area was then calculated for each month and the area of the final design was chosen to be the biggest area calculated.

Table 2-2: Different Design Method Results Summary

\begin{tabular}{|c|c|c|c|c|c|c|c|c|c|c|c|}
\hline \multirow[t]{2}{*}{$\begin{array}{c}\text { CW } \\
\text { Type }\end{array}$} & \multirow[t]{2}{*}{ Param. } & \multicolumn{2}{|c|}{$\begin{array}{c}\text { Design } \\
\text { Concentration } \\
(\mathrm{mg} / \mathrm{L})\end{array}$} & \multicolumn{2}{|c|}{ Campbell } & \multicolumn{2}{|c|}{ Reed et al. } & \multicolumn{2}{|c|}{ EPA } & \multicolumn{2}{|c|}{ Kadlec } \\
\hline & & In & Out & $\begin{array}{c}\text { Area } \\
\left(\mathbf{m}^{2}\right)\end{array}$ & $\begin{array}{c}\text { HDT } \\
\text { (days) }\end{array}$ & $\begin{array}{c}\text { Area } \\
\left(\mathbf{m}^{2}\right)\end{array}$ & $\begin{array}{c}\text { HDT } \\
\text { (days) }\end{array}$ & $\begin{array}{c}\text { Area } \\
\left(\mathbf{m}^{2}\right)\end{array}$ & $\begin{array}{c}\text { DT } \\
\text { (days) }\end{array}$ & $\begin{array}{c}\text { Area } \\
\left(\mathrm{m}^{2}\right)\end{array}$ & $\begin{array}{c}\text { HDT } \\
\text { (days) }\end{array}$ \\
\hline \multirow[t]{4}{*}{ SSF } & BOD & 74 & 40 & 349 & 0.7 & 309 & 0.7 & 5,549 & 16.8 & 173 & 0.4 \\
\hline & TSS & 147 & 40 & 76 & 0.2 & - & - & 888 & 2.7 & 56 & 0.1 \\
\hline & Total N & 40 & 40 & - & - & - & - & - & - & 2,467 & 5.2 \\
\hline & Total P & 5 & 5 & - & - & 243 & 0.5 & - & - & 106 & 0.2 \\
\hline \multirow[t]{4}{*}{ SF } & BOD & 40 & 20 & 1,356 & 3.3 & 45 & 0.1 & 537 & 1.6 & 1,130 & 2.8 \\
\hline & TSS & 40 & 20 & 6.3 & 0.2 & - & - & 2,416 & 7.3 & 53 & 0.1 \\
\hline & Total N & 40 & 10 & - & - & - & - & 5,086 & 15.4 & 3,122 & 7.7 \\
\hline & Total P & 5 & 3 & - & - & - & - & - & - & 1,902 & 4.7 \\
\hline
\end{tabular}

Table 2-3 lists the calculation results per month using the Kadlec equations for BOD removal from the SSF CW. The area chosen for the final design was the largest monthly area calculated. The design size of 388 square meters was controlled by wet weather conditions in late summer. There are differences in the area size of the SSF CW compared to the initial design area size of $173 \mathrm{~m}^{2}$ because during the initial design, the BOD effluent objective of the SSF CW was $40 \mathrm{mg} / \mathrm{L}$, as opposed as the final design of the SSF that had a BOD effluent objective of $20 \mathrm{mg} / \mathrm{L}$. This new BOD effluent at the outlet of the SSF CW raised the area significantly. 
Table 2-3: Results of Design Calculations for BOD Removal in SSF CW

\begin{tabular}{|c|c|c|c|c|c|}
\hline Month & $\begin{array}{c}\text { BODin } \\
(\mathrm{mg} / \mathrm{L})\end{array}$ & $\begin{array}{c}\text { BODout } \\
\text { (mg/L) }\end{array}$ & Flow (gal/d) & Area (m2) & $\begin{array}{c}\text { Hydraulic } \\
\text { Detention time } \\
\text { (days) }\end{array}$ \\
\hline January & 135 & 20 & 16,246 & 322 & 1.26 \\
\hline February & 142 & 20 & 15,371 & 316 & 1.30 \\
\hline March & 147 & 20 & 14,831 & 312 & 1.34 \\
\hline April & 134 & 20 & 16,343 & 323 & 1.25 \\
\hline May & 100 & 20 & 22,195 & 357 & 1.02 \\
\hline June & 76 & 20 & 29,294 & 384 & 0.83 \\
\hline July & 74 & 20 & 30,437 & 387 & 0.81 \\
\hline August & 74 & 20 & 30,437 & 387 & 0.81 \\
\hline September & 73 & 20 & 30,755 & 388 & 0.80 \\
\hline October & 95 & 20 & 23,365 & 362 & 0.98 \\
\hline November & 121 & 20 & 18,157 & 334 & 1.17 \\
\hline December & 131 & 20 & 16,733 & 325 & 1.23 \\
\hline
\end{tabular}

Table 2-4 lists the calculation results per month using the Kadlec equations for Total N removal from the SF CW. The controlling conditions for nitrogen removal were in January resulting in a design area of $2,316 \mathrm{~m}^{2}$, due to the lower temperature encountered in the winter. 
Table 2-4: Results of Design Calculations for Total $N$ Removal in SF CW

\begin{tabular}{|c|c|c|c|c|c|}
\hline Month & $\operatorname{Nin}(\mathrm{mg} / \mathrm{L})$ & $\begin{array}{c}\text { Nout } \\
(\mathrm{mg} / \mathrm{L})\end{array}$ & Flow (gal/d) & Area (m2) & $\begin{array}{c}\text { Hydraulic } \\
\text { Detention time } \\
\text { (days) }\end{array}$ \\
\hline January & 49 & 10 & 16,021 & 2,316 & 13.75 \\
\hline February & 51 & 10 & 15,116 & 2,073 & 13.04 \\
\hline March & 52 & 10 & 14,557 & 2,020 & 13.20 \\
\hline April & 46 & 10 & 16,122 & 1,810 & 10.68 \\
\hline May & 35 & 10 & 22,178 & 1,873 & 8.03 \\
\hline June & 28 & 10 & 29,525 & 2,203 & 7.10 \\
\hline July & 27 & 10 & 30,708 & 2,237 & 6.93 \\
\hline August & 27 & 10 & 30,708 & 2,198 & 6.81 \\
\hline September & 27 & 10 & 31,037 & 2,190 & 6.71 \\
\hline October & 34 & 10 & 23,389 & 2,019 & 8.21 \\
\hline November & 43 & 10 & 17,999 & 2,150 & 11.36 \\
\hline December & 47 & 10 & 16,525 & 2,097 & 12.07 \\
\hline
\end{tabular}

A safety factor of additional $17 \%$ and $4 \%$ was added to the SSF CW and SF CW design areas, respectively should any of the assumptions of values used in the calculations turn out to be incorrect or should conditions other than those anticipated develop. The total SSF CW area was approximately $454 \mathrm{~m}^{2}\left(151.2 \mathrm{~m}^{2}\right.$ each cell), and the total SF CW area was approximately $2,400 \mathrm{~m}^{2}\left(799.6 \mathrm{~m}^{2}\right.$ each cell).

\subsubsection{Plant Selection}

The ideal plants would be hardy, fast growing native wetland plants adapted to the climate near the base. El Salvador did not have any plant nurseries that grow or sell 
commercial quantities of wetland plants, thus natural sources of these plants were located and the plants harvested for transplanting in the treatment wetlands. Preliminary assessments of wetland plants in the vicinity of the site were made during the two site visits above mentioned and several potential sources were found. In addition, the topic of wetland plants was discussed with several contractors, universities and governmental agencies. It was determined that the most effective means of finding and collecting these plants was to work with a local contractor or agency that knows the local situation and can work with land owners to arrange for removal of these plants. After the plants were selected with assistance of CENTA, CENTA was hired to start a nursery to grow the plants in a more controlled environment until they reached the minimum size required for constructed wetlands.

Three types of plants were used in the CW system: (a) grass-type herbaceous plants such as Brachiaria mutica and Phragmites sp.; (b) emergent herbaceous plants such as Thalia, Typha, and Cyperus species; and (c) submerged aquatic plant such as Elodea spp.

The CW system was divided into three cells, each of which was home to a combination of different species: Phragmites australis - Typha angustifolia (Cell 1-2-5), Thalia geniculata - Thalia geniculata (Cell 1-3-6) and Brachiaria mutica-Cyperus alternifolius (Cell 1-4-7). Phragmites is the most used plant around the world in CW systems, and Typha is another commonly used species (Vymazal, 2011). Brachiaria mutica grows rapidly, achieve and maintain high plant and biomass densities below the water surface, which supports desirable microbial growth. Thalia is a widespread tropical plant with the high potential for nitrogen and phosphorus removal (Polomski et. al, 2008) 
not typically used in constructed wetland applications. Thalia and Brachiaria species were used to compare their pollutants removal efficiencies with a traditional wetland-type plant used in constructed wetlands, such as, Phragmites. Towards the end of the study, Lemna spp. was observed to take over the OW, effectively eliminating the growth of Elodea spp.

Cell 1-2-5 was the control CW cell as it had typical wetland plants. The plants in the other two cells were chosen because they were easily found in wetlands near the study area. The plant arrangement was similar on Cell 1-2-5 (control cell), and Cell 1-4-7, where grass type plant was followed by emergent herbaceous plant. The arrangement on Cell 1-3-6 was different than the other two, having emergent herbaceous plant in both SSF and SF CWs. Thalia $s p$. was very abundant in the wetlands near the study area, and it was placed in both CWs in order to evaluate its treatment capacity for both types (SSF and SF CW).

Table 2-5 below summarizes the CW System plants combinations. The flow passed through each of the SSF CW in parallel, then each SSF CW cell was connected in series to one individual SF CW cell that were in parallel. Figure 2-6 illustrates the plants distribution throughout the $\mathrm{CW}$ system. 
Table 2-5: CW Cells Plant Identification

\begin{tabular}{|l|l|}
\hline Monitoring Point ID & Description \\
\hline SSF 1 & Basin 1 Outlet (SSF Inlet) \\
\hline SSF 1-2 & SSF Outlet 2 (Phragmites) from Basin 1 \\
\hline SSF 1-3 & SSF Outlet 3 (Thalia) from Basin 1 \\
\hline SSF 1-4 & SF Outlet 5 (Typha) from SSF Outlet 2 (Phragmites) \\
\hline SF 2-5 & SF Outlet 6 (Thalia) from SSF Outlet 3 (Thalia) \\
\hline SF 3-6 & SF Outlet 7 (Cyperus) from SSF Outlet 4 (Brachiaria) \\
\hline SF 4-7
\end{tabular}

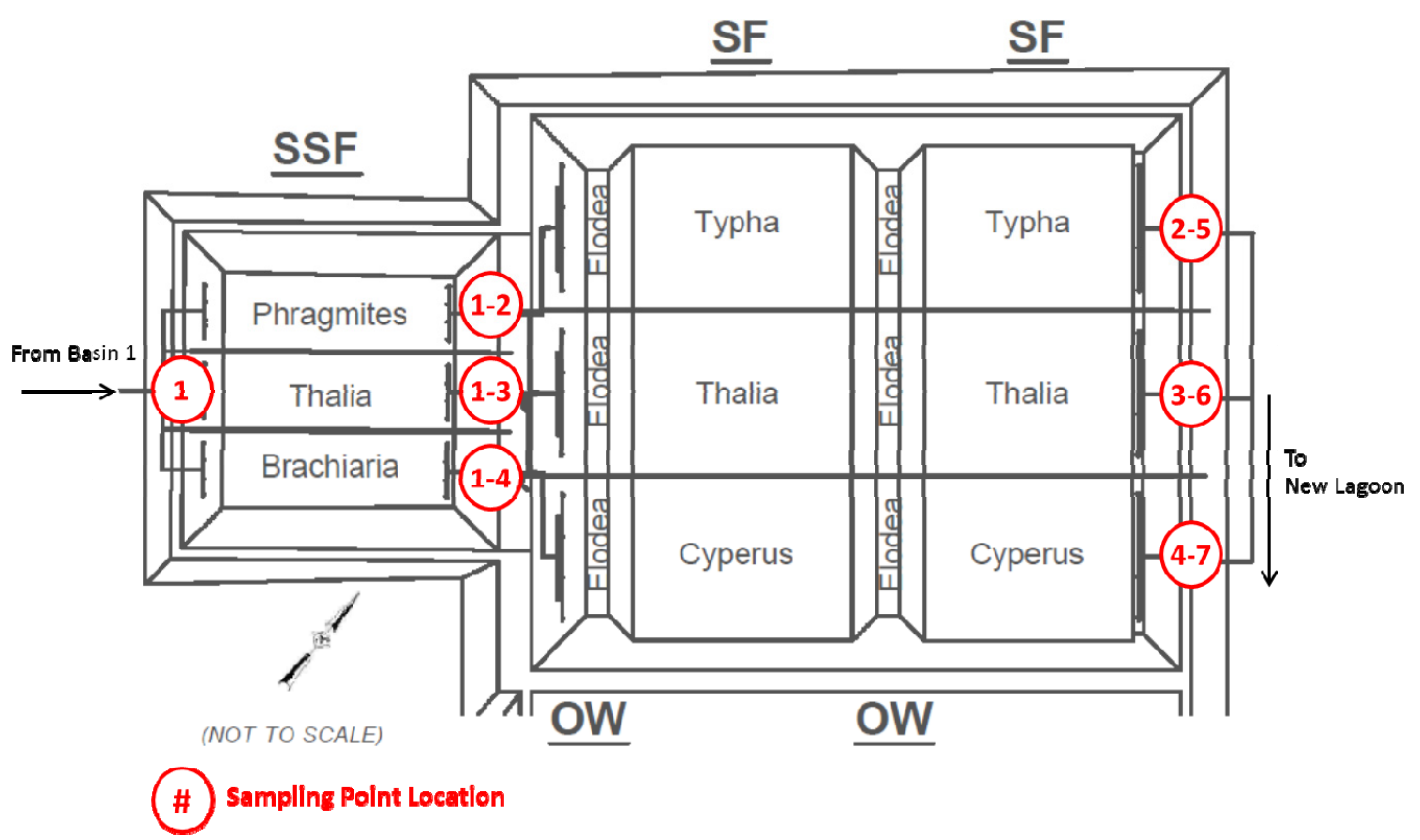

Figure 2-6: SSF and SF CW Layout, Plants Distribution, and Monitoring Points 


\subsubsection{Construction}

The wetland system construction work was undertaken from April to September 2006 by a local contractor, and construction administration also by a local company on a daily basis, with FIU personnel performing monthly site inspections. CENTA was hired to identify and test the required soil materials, and to obtain, collect and transplant the wetland plants required.

\subsection{Data Collection}

The system was monitored for eleven (11) months, from November 2006 to September 2007, with a total of seventeen (17) monitoring events. Each monitoring event consisted of visual observation, water sample collection, field parameter measurement, water quality analyses, water levels recording, monitoring for plant condition and growth rates. Grab samples were collected by scientists from CENTA ("Centro Nacional de Tecnologia Agropecuaria y Forestal") from level control boxes at the inlet and outlet of each CW cell, and were taken to a local laboratory (FUSADES) for physical/chemical analysis. Table 2-6 presents the actual monitoring event dates. Five (5) out of seventeen (17) monitoring events did not include visual observation, nor field parameter measurements. 
Table 2-6: Monitoring Event Dates

\begin{tabular}{|c|c|c|c|}
\hline $\begin{array}{l}\text { Monitoring } \\
\text { Event No. }\end{array}$ & $\begin{array}{l}\text { Monitoring Event } \\
\text { Date }\end{array}$ & $\begin{array}{l}\text { Monitoring } \\
\text { Event No. }\end{array}$ & $\begin{array}{l}\text { Monitoring Event } \\
\text { Date }\end{array}$ \\
\hline $1 *$ & November 29, 2006 & 10 & May 09, 2007 \\
\hline 2 & December 19, 2006 & 11 & June 27, 2007 \\
\hline 3 & January 08, 2007 & 12 & July 04, 2007 \\
\hline 4 & January 17, 2007 & $13^{*}$ & July 11, 2007 \\
\hline 5 & January 24, 2007 & 14 & July 18, 2007 \\
\hline 6 & January 30, 2007 & $15^{*}$ & July 25, 2007 \\
\hline 7 & February 07, 2007 & 16 & August 15, 2007 \\
\hline $8^{*}$ & March 13, 2007 & 17 & September 19, 2007 \\
\hline $9^{*}$ & April 24, 2007 & & \\
\hline \multicolumn{4}{|c|}{ * Physical/chemical analysis only } \\
\hline \multicolumn{4}{|c|}{ Other dates were complete monitoring events. } \\
\hline
\end{tabular}

Table 2-5 and Figure 2-6 show seven (7) level control structures along SSF and SF cells, with their monitoring points properly identified.

Analytical parameters for monitoring were chosen for adequately characterizing the quality of effluent domestic wastewater from the Cavalry base. Table 2-7 presents the list of the water quality parameters analyzed by FUSADES (a local laboratory) according to the standard methods, using EPA protocols: 
Table 2-7: Water Quality Analytical Parameters (by FUSADES)

\begin{tabular}{|l|l|l|}
\hline \multicolumn{2}{|l|}{ Physical-Chemical } & Microbiological \\
\hline $\begin{array}{l}\text { Biological Oxygen Demand (BOD } \\
\text { soluble) }\end{array}$ & Total Dissolved Solids (TDS) & $\begin{array}{l}\text { Fecal coliforms, (MPN/100ml } \\
\text { \& membrane filter) }\end{array}$ \\
\hline $\begin{array}{l}\text { Chemical Oxygen Demand (COD } \\
\text { soluble) }\end{array}$ & Total Nitrogen & Specific \\
\hline Total Suspended Solids (TSS) & Total phosphorus as P & Oil and Grease \\
\hline
\end{tabular}

\subsubsection{Water Balance}

The SSF inlet flow rate (Qin) was calculated based on field measured instantaneous flow rates (Qinst) and recorded pump runtime (PR):

$$
Q_{\text {inSSF }}=Q_{\text {inst. }} * P R
$$

Where: $\quad Q_{\text {inSSF }}=$ SSF inlet flow rate, $\mathrm{m}^{3} / \mathrm{d}$

$$
\begin{aligned}
& \text { Q inst }=\text { SSF inlet instantaneous flow rate, } \mathrm{m}^{3} / \mathrm{d} \\
& \text { PR = Pump Runtime, } \mathrm{h} / \mathrm{d}
\end{aligned}
$$

The SSF outlet flow rate (Qout) was calculated as the SSF inlet flow rate plus recorded Precipitation $\left(\operatorname{Pr}_{\mathrm{SSF}}\right)$ minus estimated evaporation $\left(\mathrm{ET}_{\mathrm{SSF}}\right) . \operatorname{Pr}_{\mathrm{SSF}}$ and $\mathrm{ET}_{\mathrm{SSF}}$ were calculated based on the area $\left(\mathrm{A}_{\mathrm{SSF}}\right)$ of the SSF CW.

$$
Q_{\text {ou } S S F}=Q_{i n}+\operatorname{Pr}_{S S F}-E T_{S S F}
$$

Where: $\quad Q_{\text {outSSF }}=\mathrm{SSF}$ outlet flow rate, $\mathrm{m}^{3} / \mathrm{d}$

$$
\mathrm{Q}_{\text {inSSF }}=\mathrm{SSF} \text { inlet flow rate, } \mathrm{m}^{3} / \mathrm{d}
$$




$$
\begin{aligned}
& \operatorname{Pr}_{\mathrm{SSF}}=\text { Precipitation rate }(\mathrm{m} / \mathrm{d}) * \mathrm{~A}_{\mathrm{SSF}}\left(\mathrm{m}^{2}\right), \mathrm{m}^{3} / \mathrm{d} \\
& \mathrm{ET}_{\mathrm{SSF}}=\text { Evaporation rate }(\mathrm{m} / \mathrm{d}) * \mathrm{~A}_{\mathrm{SSF}}\left(\mathrm{m}^{2}\right), \mathrm{m}^{3} / \mathrm{d} \\
& \mathrm{A}_{\mathrm{SSF}}=\mathrm{SSF} \text { CW Area, } \mathrm{m}^{2}
\end{aligned}
$$

Hydraulic detention time (HDT) was calculated based on SSF outlet flow rate, area, depth, and media porosity.

$$
H D T_{\text {cellsSF }}=\frac{\left(A_{\text {SSFcell }} * D_{\text {SSF }} * n\right)}{Q_{\text {outcell }}}
$$

Where: $\quad$ HDT $_{\text {cellsSF }}=$ Hydraulic Detention Time per SSF cell, days

$$
\begin{aligned}
& A_{S S F c e l l}=\text { SSF Area per Cell } \\
& D_{\text {SSF }}=\text { SSF Average Water Depth } \\
& \mathrm{n}=\text { Gravel Porosity } \\
& Q_{\text {outcellSSF }}=Q_{\text {outSSF }} / 3=\mathrm{SSF} \text { outlet flow rate per cell, } \mathrm{m}^{3} / \mathrm{d}
\end{aligned}
$$

Table 2-8 and Table 2-9 present the values used in the water balance calculations. The calculated SSF outlet flow rate (Qout $t_{\mathrm{SSF}}$ ) was considered to be the same for the three (3) SSF Cells, without taking into consideration different transpiration rates through the plants. 
Table 2-8: Water Balance Values per SSF Cell

\begin{tabular}{|c|c|c|c|c|c|c|c|}
\hline $\begin{array}{l}\text { Sampling } \\
\text { Date }\end{array}$ & $\begin{array}{c}\text { Qinst, } \\
\mathbf{m}^{3} / \mathbf{d}\end{array}$ & $\begin{array}{l}\text { PR, } \\
\text { h/d }\end{array}$ & $\underset{\left(\text { Qinst }^{*} \mathbf{P R}\right), \mathbf{m}^{3} / \mathbf{d}}{\operatorname{Qin}_{\mathrm{SSF}}}$ & $\begin{array}{c}\mathbf{P r}_{\text {SSF }} \\
\left(\mathbf{P r}^{* * \mathbf{A}_{\text {SSF }}}\right), \\
\mathbf{m}^{3} / \mathbf{d}\end{array}$ & $\begin{array}{c}\mathbf{E T}_{\mathrm{SSF}} \\
\left(\mathbf{E T} * \mathbf{A}_{\mathbf{S S F}}\right), \\
\mathbf{m}^{3} / \mathbf{d}\end{array}$ & $\begin{array}{l}\text { Qout }_{\text {SSF }} \\
\left(\mathbf{Q i n}_{\text {Pr. }}\right. \\
\left.\mathbf{E T}_{\text {SSF }}\right) \\
\left(\mathbf{m}^{3} / \mathbf{d}\right)\end{array}$ & $\begin{array}{c}\text { HDT }_{\text {SSF }} \\
\left(\left(A^{*} D^{*} n\right) / \text { Qout }\right) \\
\text { days }\end{array}$ \\
\hline $11 / 29 / 06$ & 72.09 & 6 & 18.02 & 0.26 & 0.69 & 17.60 & 2.06 \\
\hline $12 / 19 / 06$ & 72.09 & 4 & 12.02 & 0.17 & 0.69 & 11.50 & 3.16 \\
\hline $01 / 08 / 07$ & 72.09 & 3 & 9.01 & 0.00 & 0.71 & 8.30 & 4.37 \\
\hline $01 / 17 / 07$ & 72.09 & 3 & 9.01 & 0.00 & 0.71 & 8.30 & 4.37 \\
\hline $01 / 24 / 07$ & 72.09 & 3 & 9.01 & 0.00 & 0.71 & 8.30 & 4.37 \\
\hline 01/30/07 & 72.09 & 3 & 9.01 & 0.00 & 0.71 & 8.30 & 4.37 \\
\hline $02 / 07 / 07$ & 72.09 & 3 & 9.01 & 0.00 & 0.83 & 8.18 & 4.44 \\
\hline 03/13/07 & 72.09 & 3 & 9.01 & 0.03 & 0.93 & 8.12 & 4.47 \\
\hline $04 / 24 / 07$ & 72.09 & 3 & 9.01 & 0.43 & 0.88 & 8.56 & 4.24 \\
\hline 05/09/07 & 72.09 & 3 & 9.01 & 0.73 & 0.75 & 8.99 & 4.04 \\
\hline $06 / 27 / 07$ & 72.09 & 4 & 12.02 & 0.37 & 0.63 & 11.75 & 3.09 \\
\hline 07/04/07 & 72.09 & 5 & 15.02 & 1.70 & 0.70 & 16.02 & 2.27 \\
\hline $07 / 11 / 07$ & 72.09 & 6 & 18.02 & 1.70 & 0.70 & 19.02 & 1.91 \\
\hline 07/18/07 & 72.09 & 6 & 18.02 & 1.70 & 0.70 & 19.02 & 1.91 \\
\hline $07 / 25 / 07$ & 72.09 & 8 & 24.03 & 1.70 & 0.70 & 25.03 & 1.45 \\
\hline $08 / 15 / 07$ & 72.09 & 8 & 24.03 & 1.44 & 0.75 & 24.72 & 1.47 \\
\hline 09/19/07 & 72.09 & 12 & 36.05 & 1.52 & 0.67 & 36.90 & 0.98 \\
\hline Average & 72.09 & 4.88 & 14.67 & 0.69 & 0.73 & 14.63 & 3.11 \\
\hline $\begin{array}{l}\text { Standard } \\
\text { Deviation }\end{array}$ & 0.00 & 2.47 & 7.42 & 0.72 & 0.07 & 7.99 & 1.25 \\
\hline \multicolumn{8}{|c|}{$\begin{array}{l}\text { Notes: } Q_{\text {inst }}=\text { Instantaneous Flow; } P R=\text { Pump Runtime; } P r=\text { Precipitation; } \text { ET }=\text { Evapotranspiration; } \\
H D T_{S S F}=\text { Hydraulic Detention Time. } A_{S S F}=151.2 \mathrm{~m}^{2} ; \text { Water Depth }=0.6 \mathrm{~m} ; \text { Gravel Porosity }=0.4 \\
\mathrm{~m}^{3} / \mathrm{m}^{3}\end{array}$} \\
\hline
\end{tabular}

The SF inlet flow rate (Qin $\left.{ }_{\mathrm{SF}}\right)$ was the SSF calculated outlet flow rate. The SF outlet flow rate $($ Qout $\mathrm{SF}$ ) was calculated as the SF inlet flow rate plus recorded Precipitation 
$\left(\mathrm{Pr}_{\mathrm{SF}}\right)$ minus estimated evaporation $\left(\mathrm{ET}_{\mathrm{SF}}\right)$. $\mathrm{Pr}_{\mathrm{SF}}$ and $\mathrm{ET}_{\mathrm{SF}}$ were calculated based on the area $\left(\mathrm{A}_{\mathrm{SF}}\right)$ of the SF CW.

Table 2-9: Water Balance Values per SF Cell

\begin{tabular}{|c|c|c|c|c|c|}
\hline Sampling Date & $\begin{array}{l}\text { Qin }_{\text {SF }} \\
\left(\text { Qout }_{\text {SSF }}+\text { Pr }_{\text {SSF}^{-}}\right. \\
\left.\text {ET }_{\text {SSF }}\right)\left(\mathbf{m}^{3} / \mathbf{d}\right)\end{array}$ & $\begin{array}{c}\operatorname{Pr}_{\mathrm{SF}}\left(\operatorname{Pr}_{\mathbf{m}^{3} / \mathbf{d}} \mathbf{A}_{\mathrm{SF}}\right) \\
\mathbf{m}\end{array}$ & $\begin{array}{l}\mathbf{E T}_{\mathrm{SF}} \\
\underset{\mathbf{E T}}{\left.\mathbf{E} \mathbf{A}_{\mathbf{S F}}\right)} \\
\mathbf{m}^{\mathbf{3}} / \mathbf{d}\end{array}$ & $\begin{array}{c}\text { Qout }_{\text {SF }} \\
\left(\mathbf{Q i n}_{\mathrm{SF}}+\mathbf{P r}_{\mathrm{SF}^{-}}\right. \\
\left.\mathbf{E T}_{\mathrm{SF}}\right)\left(\mathbf{m}^{3} / \mathbf{d}\right)\end{array}$ & $\begin{array}{l}\text { HDT }_{\text {SF }} \\
((\mathbf{A} * \mathbf{D} * \mathbf{n}) / \text { Qout }) \\
\quad \text { days }\end{array}$ \\
\hline $11 / 29 / 06$ & 17.60 & 1.39 & 3.63 & 15.36 & 23.70 \\
\hline $12 / 19 / 06$ & 11.50 & 0.90 & 3.64 & 8.76 & 41.54 \\
\hline 01/08/07 & 8.30 & 0.00 & 3.74 & 4.56 & 79.77 \\
\hline $01 / 17 / 07$ & 8.30 & 0.00 & 3.74 & 4.56 & 79.77 \\
\hline $01 / 24 / 07$ & 8.30 & 0.00 & 3.74 & 4.56 & 79.77 \\
\hline $01 / 30 / 07$ & 8.30 & 0.00 & 3.74 & 4.56 & 79.77 \\
\hline $02 / 07 / 07$ & 8.18 & 0.00 & 4.40 & 3.78 & 96.27 \\
\hline $03 / 13 / 07$ & 8.12 & 0.18 & 4.90 & 3.40 & 107.13 \\
\hline $04 / 24 / 07$ & 8.56 & 2.28 & 4.66 & 6.17 & 58.99 \\
\hline 05/09/07 & 8.99 & 3.87 & 3.97 & 8.89 & 40.96 \\
\hline $06 / 27 / 07$ & 11.75 & 1.95 & 3.33 & 10.37 & 35.11 \\
\hline 07/04/07 & 16.02 & 9.00 & 3.71 & 21.31 & 17.09 \\
\hline $07 / 11 / 07$ & 19.02 & 9.00 & 3.71 & 24.31 & 14.98 \\
\hline $07 / 18 / 07$ & 19.02 & 9.00 & 3.71 & 24.31 & 14.98 \\
\hline $07 / 25 / 07$ & 25.03 & 9.00 & 3.71 & 30.32 & 12.01 \\
\hline $08 / 15 / 07$ & 24.72 & 7.64 & 3.97 & 28.39 & 12.83 \\
\hline $09 / 19 / 07$ & 36.90 & 8.05 & 3.52 & 41.43 & 8.79 \\
\hline Average & 14.63 & 3.66 & 3.86 & 14.43 & 46.84 \\
\hline Standard Deviation & 7.99 & 3.80 & 0.38 & 11.34 & 31.97 \\
\hline \multicolumn{6}{|c|}{$\begin{array}{l}\text { Notes: } \text { Pr }=\text { Precipitation; ET }=\text { Evapotranspiration; HDT }=\text { Hydraulic Detention Time. } \\
A_{S F}=605 \mathrm{~m}^{2} ; \text { Water Depth }=0.45 \mathrm{~m} ; \text { Root Porosity }=0.65 \mathrm{~m}^{3} / \mathrm{m}^{3} \\
A_{O W}=195 \mathrm{~m}^{2} ; \text { Water Depth }=1.2 \mathrm{~m} ; \text { Root Porosity }=0.8 \mathrm{~m}^{3} / \mathrm{m}^{3}\end{array}$} \\
\hline
\end{tabular}


As there was a great variation in flow and hydraulic detention time during the wet and dry season, the water balance data presented above was re-organized by wet and dry season in Table 2-10 and Table 2-11. Wet season includes the months of May to November, and dry season includes the months of December to April.

Table 2-10: Seasonal Water Balance Values per SSF Cell

\begin{tabular}{|c|c|c|c|c|c|c|c|c|}
\hline \multicolumn{9}{|c|}{ Seasonal Water Balance Values per SSF Cell } \\
\hline Season & Date & $\begin{array}{c}\text { Qinst, } \\
\mathbf{m}^{3} / \mathbf{d}\end{array}$ & $\begin{array}{l}\text { PR, } \\
\text { h/d }\end{array}$ & $\begin{array}{c}\operatorname{Qin}_{\mathrm{SSF}} \\
\left(\text { Qinst*PR) }^{*} \text {, }\right. \\
\mathbf{m}^{3} / \mathbf{d}\end{array}$ & $\begin{array}{c}\operatorname{Pr}_{\mathrm{SSF}} \\
\left(\operatorname{Pr}^{*} A_{\mathrm{SSF}}\right. \\
), \mathbf{m}^{3} / \mathbf{d}\end{array}$ & $\begin{array}{c}\mathbf{E T}_{\mathrm{SSF}} \\
\left(\mathbf{E T} \mathbf{A}_{\mathrm{SSF}}\right), \\
\mathbf{m}^{\mathbf{3}} / \mathbf{d}\end{array}$ & 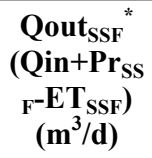 & $\begin{array}{c}\text { HDT }_{\text {SSF }} \\
\left(\left(\mathbf{A}^{*} \mathbf{D}^{*} \mathbf{n}\right)\right. \\
\text { /Qout }), \\
\text { days }\end{array}$ \\
\hline \multirow{11}{*}{$\begin{array}{l}\text { Wet } \\
\text { (May to } \\
\text { Nov) }\end{array}$} & $11 / 29 / 06$ & 72.09 & 6 & 18.02 & 0.26 & 0.69 & 17.60 & 2.06 \\
\hline & $5 / 9 / 07$ & 72.09 & 3 & 9.01 & 0.73 & 0.75 & 8.99 & 4.04 \\
\hline & $6 / 27 / 07$ & 72.09 & 4 & 12.02 & 0.37 & 0.63 & 11.75 & 3.09 \\
\hline & $7 / 4 / 07$ & 72.09 & 5 & 15.02 & 1.70 & 0.70 & 16.02 & 2.27 \\
\hline & $7 / 11 / 07$ & 72.09 & 6 & 18.02 & 1.70 & 0.70 & 19.02 & 1.91 \\
\hline & $7 / 18 / 07$ & 72.09 & 6 & 18.02 & 1.70 & 0.70 & 19.02 & 1.91 \\
\hline & $7 / 25 / 07$ & 72.09 & 8 & 24.03 & 1.70 & 0.70 & 25.03 & 1.45 \\
\hline & $8 / 15 / 07$ & 72.09 & 8 & 24.03 & 1.44 & 0.75 & 24.72 & 1.47 \\
\hline & $9 / 19 / 07$ & 72.09 & 12 & 36.05 & 1.52 & 0.67 & 36.90 & 0.98 \\
\hline & AVE & 72.09 & 6.44 & 19.36 & 1.24 & 0.70 & 19.90 & 2.13 \\
\hline & SD & 0.00 & 2.65 & 7.96 & 0.61 & 0.04 & 8.26 & 0.93 \\
\hline \multirow{10}{*}{$\begin{array}{c}\text { Dry } \\
\text { (Dec to } \\
\text { April) }\end{array}$} & $12 / 19 / 06$ & 72.09 & 4 & 12.02 & 0.17 & 0.69 & 11.50 & 3.16 \\
\hline & $1 / 8 / 07$ & 72.09 & 3 & 9.01 & 0.00 & 0.71 & 8.30 & 4.37 \\
\hline & $1 / 17 / 07$ & 72.09 & 3 & 9.01 & 0.00 & 0.71 & 8.30 & 4.37 \\
\hline & $1 / 24 / 07$ & 72.09 & 3 & 9.01 & 0.00 & 0.71 & 8.30 & 4.37 \\
\hline & $1 / 30 / 07$ & 72.09 & 3 & 9.01 & 0.00 & 0.71 & 8.30 & 4.37 \\
\hline & $2 / 7 / 07$ & 72.09 & 3 & 9.01 & 0.00 & 0.78 & 8.23 & 4.41 \\
\hline & $3 / 13 / 07$ & 72.09 & 3 & 9.01 & 0.03 & 0.93 & 8.12 & 4.47 \\
\hline & $4 / 24 / 07$ & 72.09 & 3 & 9.01 & 0.43 & 0.88 & 8.56 & 4.24 \\
\hline & AVE & 72.09 & 3.13 & 9.39 & 0.08 & 0.76 & 8.70 & 4.22 \\
\hline & SD & 0.00 & 0.35 & 1.06 & 0.15 & 0.09 & 1.14 & 0.43 \\
\hline \multicolumn{9}{|c|}{$\begin{array}{l}\text { Notes: } Q_{\text {inst }}=\text { Instantaneous Flow; } P R=\text { Pump Runtime } ; P r=\text { Precipitation; } E T=\text { Evapotranspiration; } D T= \\
\text { Hydraulic Detention Time } ; \text { AVE }=\text { Average } ; S D=\text { Standard Deviation } \\
A_{S S F}=151.2 \mathrm{~m}^{2} ; \text { Water Depth }=0.6 \mathrm{~m} ; \text { Gravel Porosity }=0.4 \mathrm{~m}^{3} / \mathrm{m}^{3}\end{array}$} \\
\hline
\end{tabular}


As it can be seen in Table 2-10, the calculated SSF CW average influent flows during the wet and dry season were $19.36 \mathrm{~m}^{3} /$ day $\pm 7.96 \mathrm{~m}^{3} /$ day and $9.39 \mathrm{~m}^{3} /$ day $\pm 1.06 \mathrm{~m}^{3} /$ day, respectively. The calculated SSF CW average effluent flows during the wet and dry season were $19.90 \mathrm{~m}^{3} /$ day $\pm 8.26 \mathrm{~m}^{3} /$ day and $8.70 \mathrm{~m}^{3} /$ day $\pm 1.14 \mathrm{~m}^{3} /$ day, respectively. The calculated SSF CW average hydraulic detention times during the wet and dry season were 2.13 days \pm 0.93 days and 4.22 days \pm 0.43 days, respectively.

Table 2-11: Seasonal Water Balance Values per SF Cell

\begin{tabular}{|c|c|c|c|c|c|c|}
\hline Season & Date & $\begin{array}{c}\operatorname{Qin}_{\mathrm{SF}} \\
\left(\mathrm{Qin}_{\mathrm{SSF}}+\mathrm{Pr}_{\mathrm{SSF}^{-}}\right. \\
\left.\mathbf{E T}_{\mathrm{SSF}}\right)\left(\mathrm{m}^{3} / \mathrm{d}\right)\end{array}$ & $\begin{array}{c}\operatorname{Pr}_{\mathrm{SF}} \\
\left(\operatorname{Pr}^{*} \mathbf{A}_{\mathrm{SF}}\right) \\
\mathbf{m}^{\mathbf{3}} / \mathbf{d}\end{array}$ & $\begin{array}{c}\mathbf{E T}_{\mathrm{SF}} \\
\left(\mathbf{E T}{ }^{*} \mathbf{A}_{\mathrm{SF}}\right) \\
\mathbf{m}^{3} / \mathbf{d}\end{array}$ & $\begin{array}{c}\text { Qout }_{\mathrm{SF}} \\
\left(\mathbf{Q i n}+\mathbf{P r}_{\mathrm{SF}^{-}}\right. \\
\left.\mathbf{E T}_{\mathrm{SF}}\right) \\
\left(\mathbf{m}^{3} / \mathbf{d}\right)\end{array}$ & $\begin{array}{c}\text { HDT }_{\mathrm{SF}} \\
((\mathrm{A} * \mathrm{D} * \mathrm{n}) / \text { Qout }), \\
\text { days }\end{array}$ \\
\hline \multirow{11}{*}{$\begin{array}{l}\text { Wet } \\
\text { (May to } \\
\text { Nov) }\end{array}$} & $11 / 29 / 06$ & 17.60 & 1.39 & 3.63 & 15.36 & 23.70 \\
\hline & $5 / 9 / 07$ & 8.99 & 3.87 & 3.97 & 8.89 & 40.96 \\
\hline & $6 / 27 / 07$ & 11.75 & 1.95 & 3.33 & 10.37 & 35.11 \\
\hline & $7 / 4 / 07$ & 16.02 & 9.00 & 3.71 & 21.31 & 17.09 \\
\hline & $7 / 11 / 07$ & 19.02 & 9.00 & 3.71 & 24.31 & 14.98 \\
\hline & $7 / 18 / 07$ & 19.02 & 9.00 & 3.71 & 24.31 & 14.98 \\
\hline & $7 / 25 / 07$ & 25.03 & 9.00 & 3.71 & 30.32 & 12.01 \\
\hline & $8 / 15 / 07$ & 24.72 & 7.64 & 3.97 & 28.39 & 12.83 \\
\hline & 9/19/07 & 36.90 & 8.05 & 3.52 & 41.43 & 8.79 \\
\hline & AVE & 19.90 & 6.54 & 3.70 & 22.74 & 20.05 \\
\hline & SD & 8.26 & 3.21 & 0.20 & 10.28 & 11.07 \\
\hline \multirow{10}{*}{$\begin{array}{l}\text { Dry } \\
\text { (Dec to } \\
\text { April) }\end{array}$} & $12 / 19 / 06$ & 11.50 & 0.90 & 3.64 & 8.76 & 41.54 \\
\hline & $1 / 8 / 07$ & 8.30 & 0.00 & 3.74 & 4.56 & 79.77 \\
\hline & $1 / 17 / 07$ & 8.30 & 0.00 & 3.74 & 4.56 & 79.77 \\
\hline & $1 / 24 / 07$ & 8.30 & 0.00 & 3.74 & 4.56 & 79.77 \\
\hline & $1 / 30 / 07$ & 8.30 & 0.00 & 3.74 & 4.56 & 79.77 \\
\hline & $2 / 7 / 07$ & 8.23 & 0.00 & 4.14 & 4.09 & 89.07 \\
\hline & $3 / 13 / 07$ & 8.12 & 0.18 & 4.90 & 3.40 & 107.13 \\
\hline & $4 / 24 / 07$ & 8.56 & 2.28 & 4.66 & 6.17 & 58.99 \\
\hline & AVE & 8.70 & 0.42 & 4.04 & 5.08 & 76.98 \\
\hline & SD & 1.14 & 0.81 & 0.49 & 1.68 & 19.52 \\
\hline \multicolumn{7}{|c|}{$\begin{array}{l}\text { Notes: } \text { Pr }=\text { Precipitation } ; \text { ET }=\text { Evapotranspiration } ; H D T=\text { Hydraulic Detention Time; } \text { AVE }=\text { Average; } \\
S D=\text { Standard Deviation } \\
A_{S F}=605 \mathrm{~m}^{2} ; \text { Water Depth }=0.45 \mathrm{~m} ; \text { Root Porosity }=0.65 \mathrm{~m}^{3} / \mathrm{m}^{3} \\
A_{O W}=195 \mathrm{~m}^{2} ; \text { Water Depth }=1.2 \mathrm{~m} ; \text { Root Porosity }=0.8 \mathrm{~m}^{3} / \mathrm{m}^{3}\end{array}$} \\
\hline
\end{tabular}


As it can be seen in Table 2-11, the calculated SF CW average influent flows during the wet and dry season were $19.90 \mathrm{~m}^{3} /$ day $\pm 8.26 \mathrm{~m}^{3} /$ day and $8.70 \mathrm{~m}^{3} /$ day $\pm 1.14 \mathrm{~m}^{3} /$ day, respectively (same as the SSF CW average effluent flow). The calculated SF CW average effluent flows during the wet and dry season were $22.74 \mathrm{~m}^{3} /$ day $\pm 10.28 \mathrm{~m}^{3} /$ day and $5.08 \mathrm{~m}^{3} /$ day $\pm 1.68 \mathrm{~m}^{3} /$ day, respectively. The calculated SF CW average hydraulic detention times during the wet and dry season were 20.05 days \pm 11.07 days and 76.98 days \pm 19.52 days, respectively.

The actual flows in comparison with the flows estimated during design were significant lower, as shown in Figure 2-7. This difference in flow is attributed to soldiers that were assigned to overseas duties during the duration of this study.

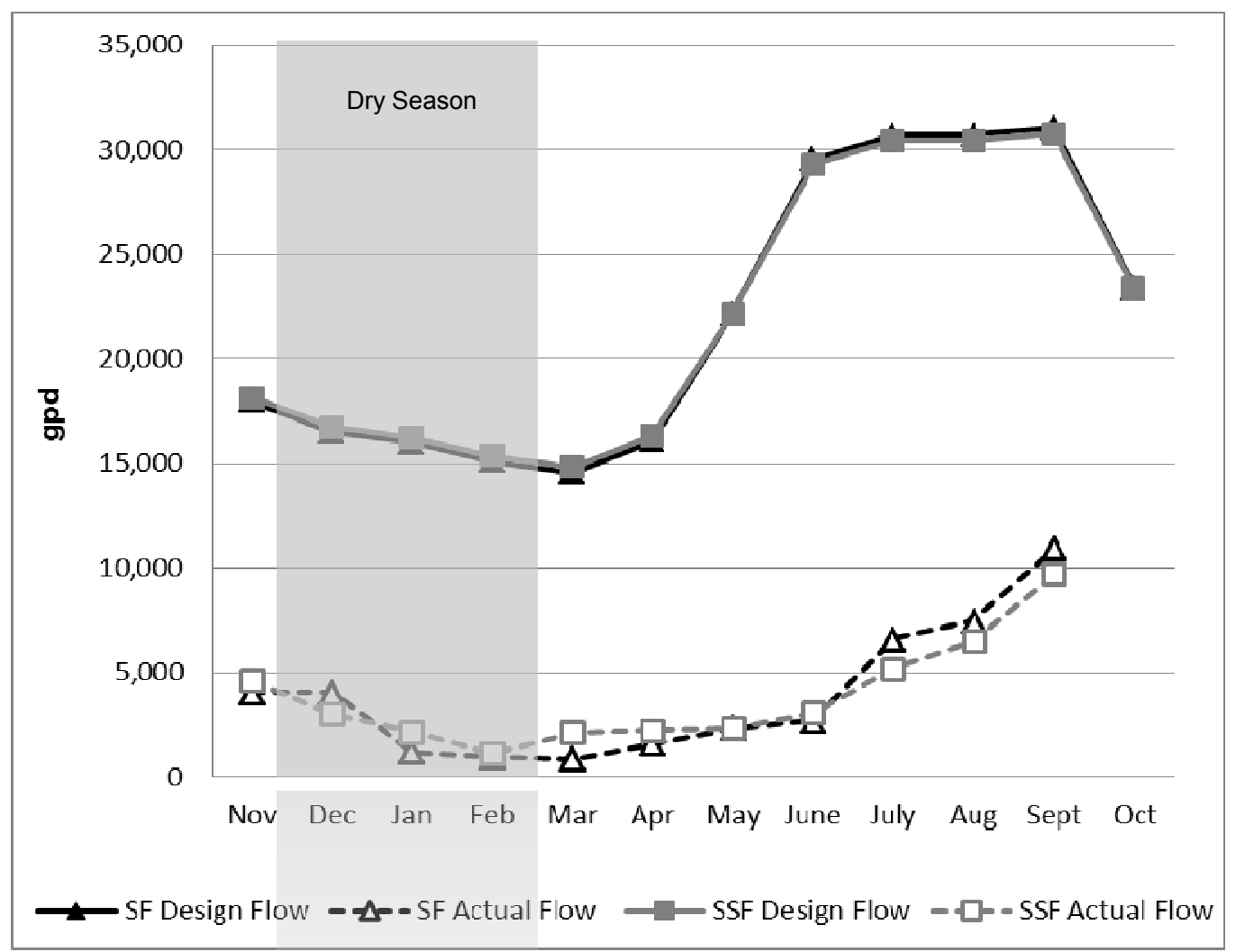

Figure 2-7: Design Flow vs. Actual Flow 


\subsubsection{Quality Indicators}

The available data from the SSF CW influent (Sampling Point 1), from the SSF CW cells effluent (Sampling Points 1-2, 1-3, and 1-4), and from the SF CW cells effluent (Sampling Points 2-5, 3-6, and 4-7) are included in Appendix B Tables B-1 to B-7. The average and standard deviation for the available SSF CW, SF CW, and overall $\mathrm{CW}$ system data are also included in Appendix B Tables B-8 to B-10, organized by parameter.

As there was a great variation in the data between the seasons, the influent and effluent data were organized by dry and wet season, and are presented in Appendix B - Tables B11 to B-24 in terms of percent (\%) residual. Percent (\%) residual was calculated using the following equation:

$\%$ Residual $=\frac{\text { Effluent Concentration }}{\text { Influent } \text { Concentration }}$

As mentioned above, wet season includes the months of May to November, and dry season includes the months of December to April.

\subsubsection{Field Parameters}

The following field parameters were measured during all the monitoring events:

- Wastewater Temperature;

- $\mathrm{pH}$;

- Oxidation Reduction Potential (ORP);

- Dissolved Oxygen (DO); and 
- Conductivity

Field parameters were measured at the following locations:

- SSF Inlet,

- SSF Outlets/SF Inlets;

- Open Water Section located in the SF Inlet (OW-A) at three different depths (surface, $0.6 \mathrm{~m}$ below the surface, and bottom);

- Open Water Section located in the middle of the SF CW (OW-B) at three different depths (surface, $0.6 \mathrm{~m}$ below the surface, and bottom);

- SF Outlets.

Figure 2-8 to Figure 2-12 present the field data average per location. The temperature dropped approximately 2 degrees Celsius in the open water sections, as shown in Figure 2-8, which can be attributed to the floating vegetation shade in those cells. The $\mathrm{pH}$ dropped through the SSF wetland and had a slight increase through the SF wetland (Figure 2-9). The oxidation reduction potential (ORP) went up as the flow was moving through the CW system (Figure 2-10), indicating less reducing conditions (Lazareva \& Pichler, 2010). The dissolved oxygen (DO) level reduced through the SSF wetland and it increased by $1 \mathrm{mg} / \mathrm{L}$ through the SF wetland (Figure 2-11). Conductivity went down as the flow went through the CW system, with higher conductivity levels at the bottom of the OW sections which could be attributed to limited water mixing and stagnant 
conditions at the bottom of the OW sections (Figure 2-12). Field parameters data is included in Appendix B Tables B-25 to B-30.

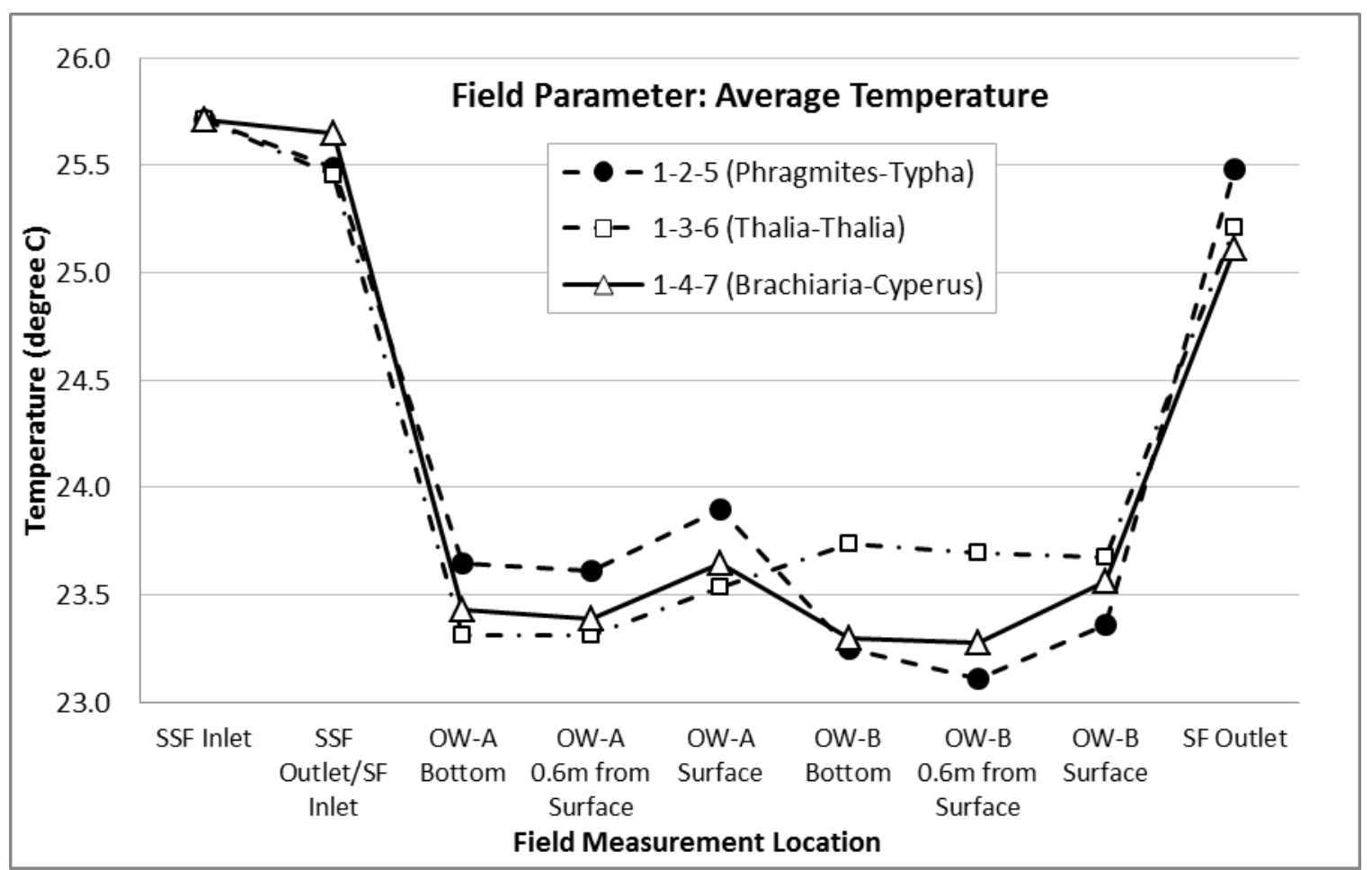

Figure 2-8: Field Parameter - Average Temperature 


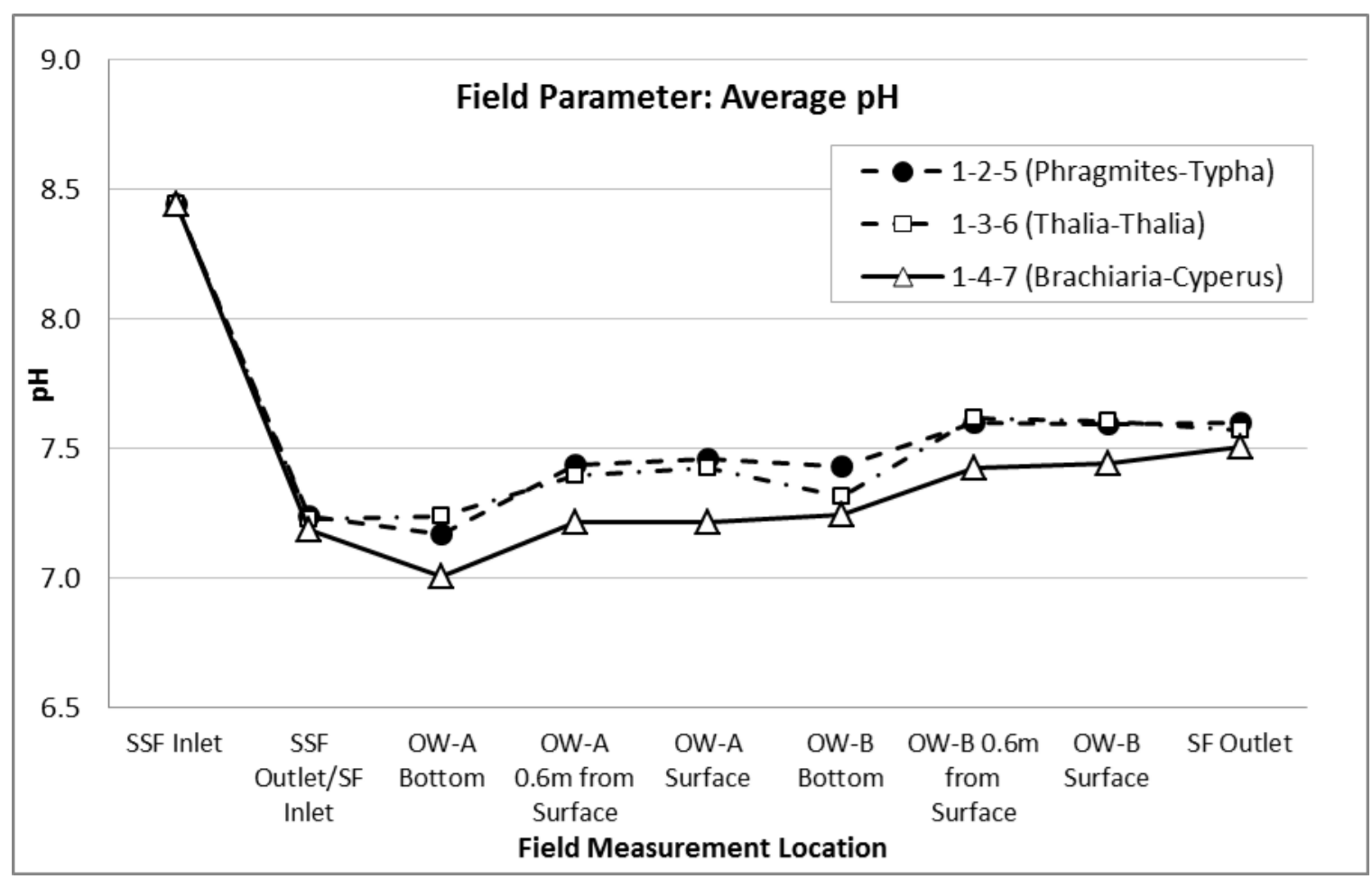

Figure 2-9: Field Parameter - Average pH

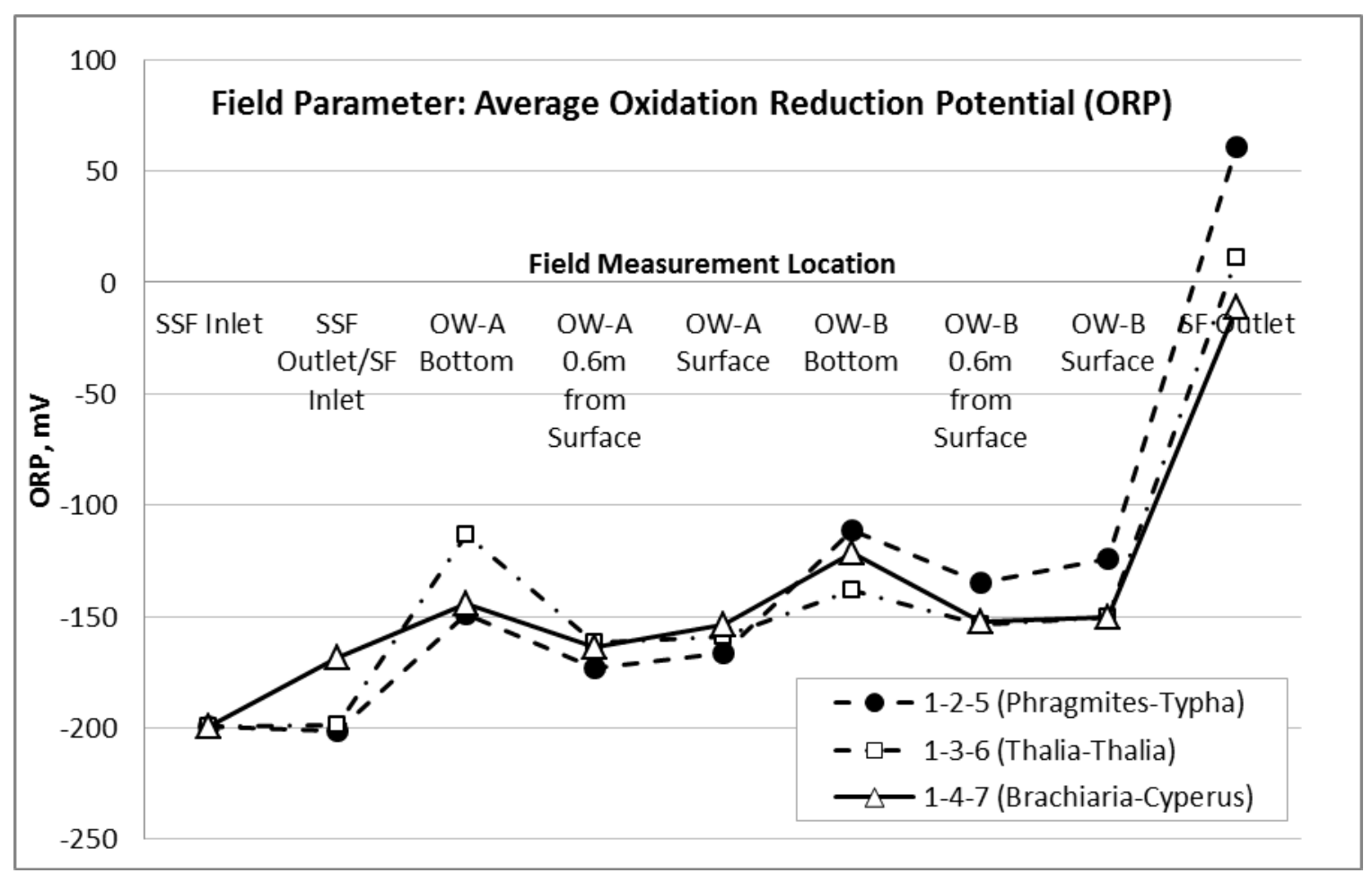

Figure 2-10: Field Parameter - Average ORP 


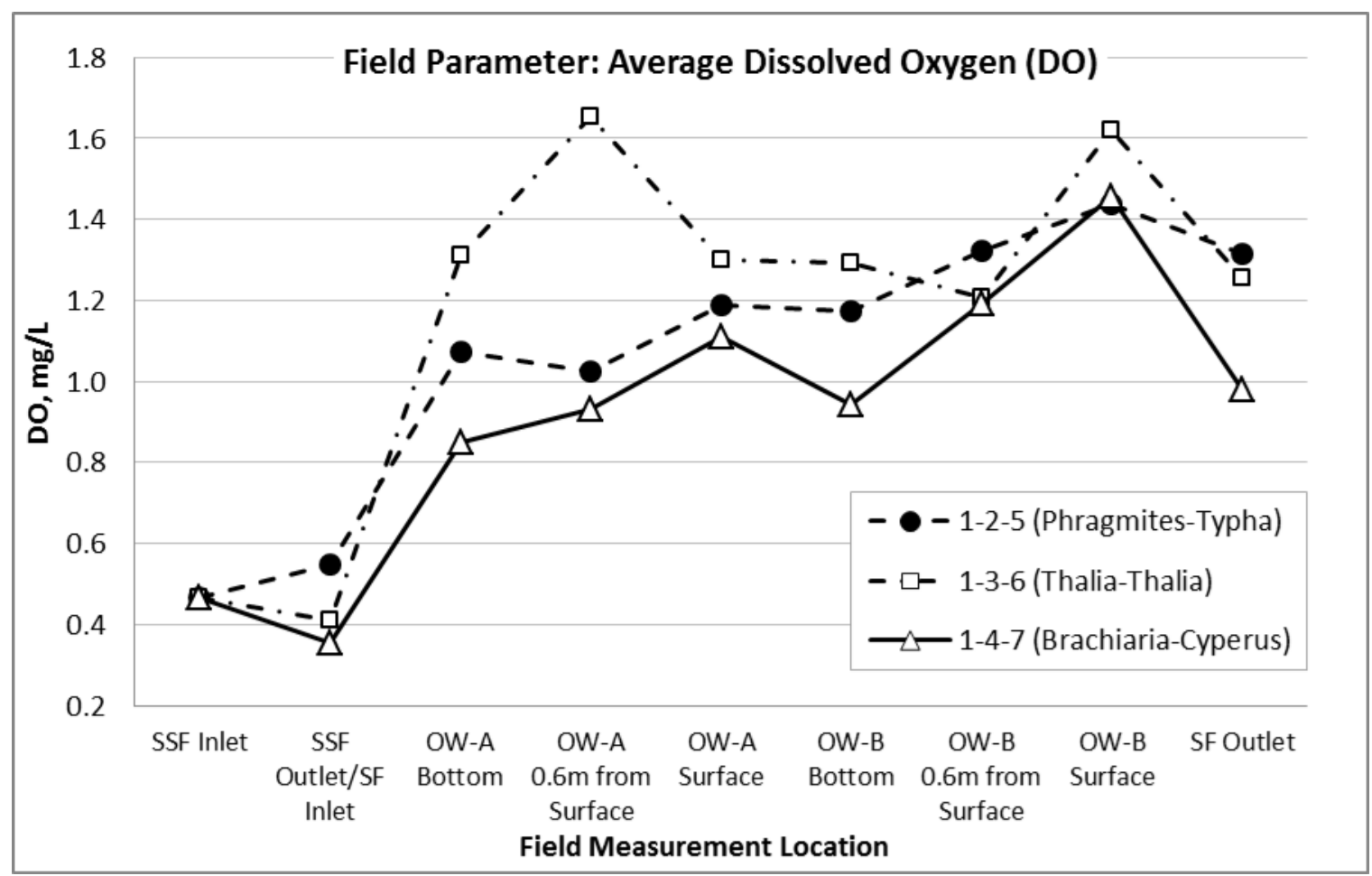

Figure 2-11: Field Parameter - Average DO

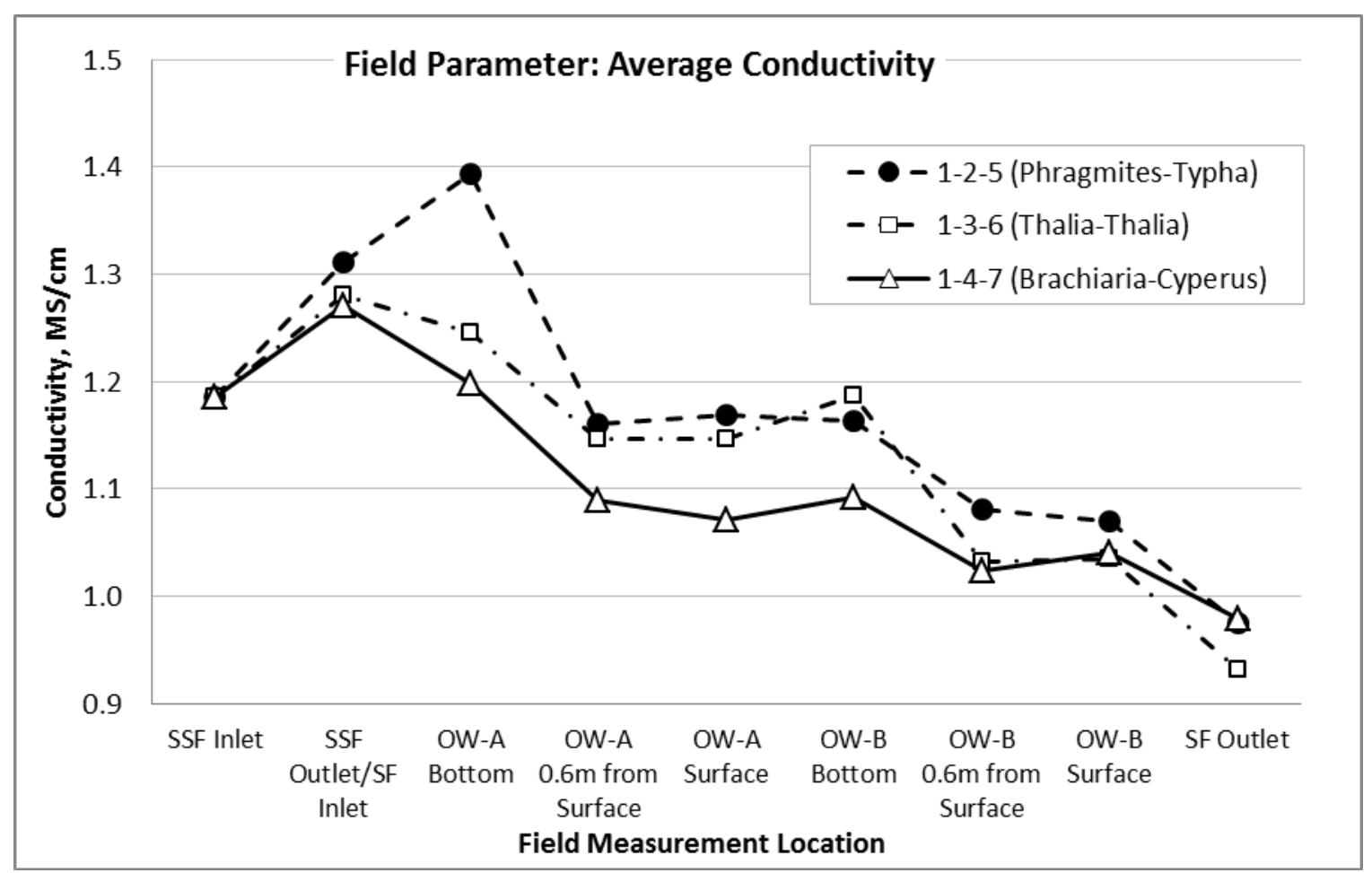

Figure 2-12: Field Parameter - Average Conductivity 


\subsection{Statistical Analysis.}

Basic statistics, such as average and standard deviation results, are presented for comparison of the selected quality indicators in the influent to and effluent from the SSF and SF, including percent removals.

All the statistical calculations were performed using Excel's data analysis tool, with random hand calculation to validate the excel results. 


\section{RESULTS AND DISCUSSION}

This Chapter presents the statistical analysis results as described above for the following:

- SSF, SF, and CW System Influent and Effluent Characterization; and

- Comparative Assessment of Models Used During Design

\subsection{Influent and Effluent Characterization}

A summary of the SSF CW, SF CW, and overall CW System performances per season and per wetland plant are presented in Appendix B, and discussed further in this Chapter for the following parameters:

- BOD;

- TSS.

- COD;

- Fecal Coliform;

- Oil \& Grease;

- Total Nitrogen; and

- Phosphorus

Three approaches are commonly encountered to assess a CW system removal performance: effluent concentrations, areal removed load, and performance mass removal 
(Hijosa-Valsero, et al., 2010). Since the design was performed based on effluent concentrations in order to achieve the target effluent objectives, the CW system of this study is assessed based on effluent concentrations, with residual percentage presented herein. The 95\% confidence levels for all the above parameters are included in Appendix B (Table B-22 to B-24).

\subsubsection{BOD $_{5}$}

$\mathrm{BOD}_{5}$ concentrations were reduced by the CW System, with the exception of one (1) monitoring event, as shown in Figure 3-1. Seasonal results are summarized below.

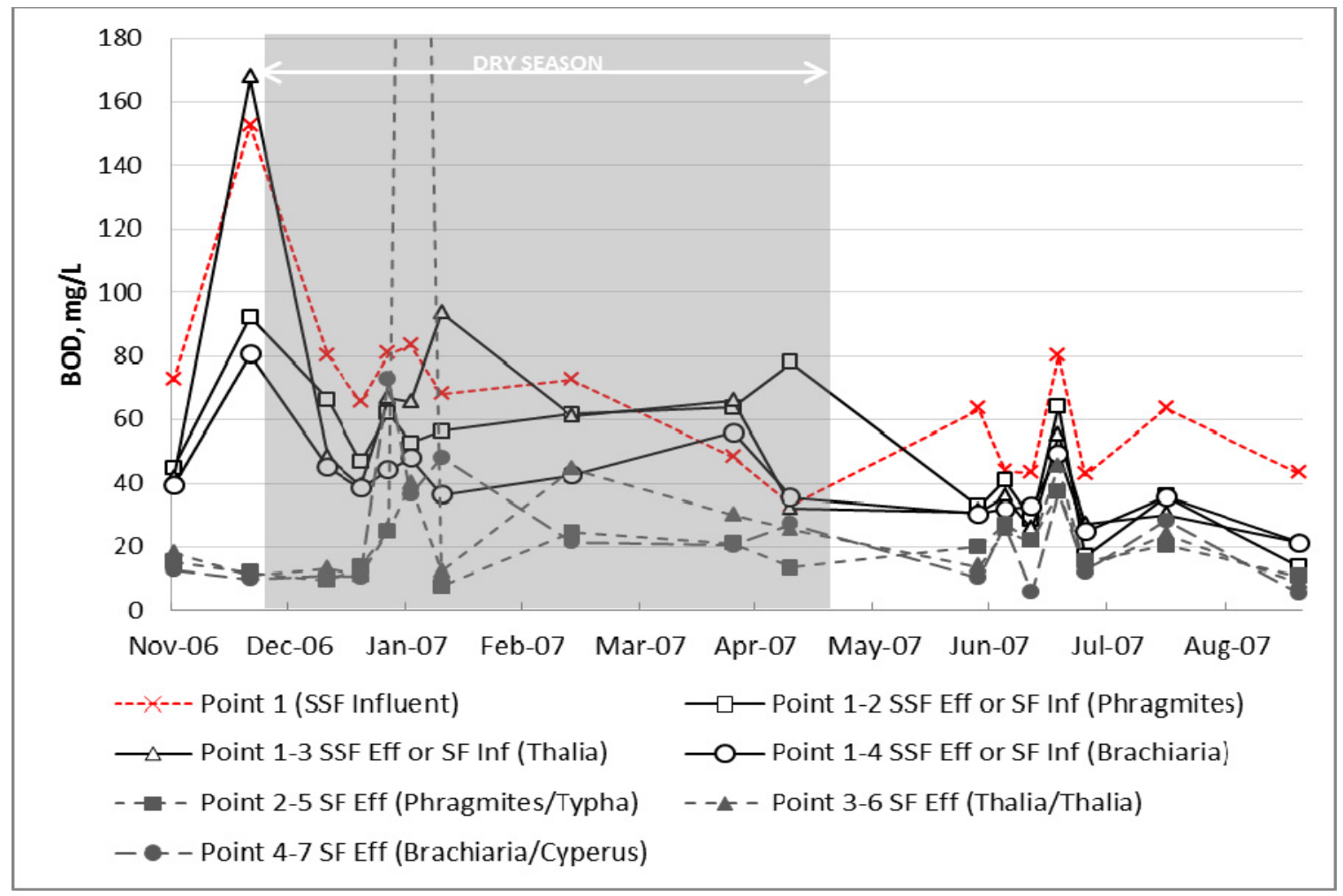

Figure 3-1: BOD $_{5}$ Influent vs. Effluent Concentrations

The $\mathrm{BOD}_{5}$ average effluent concentrations ranged from 18.5 to $21.7 \mathrm{mg} / \mathrm{L}$ during the wet season (May to November). Table 3-1 summarizes the $\mathrm{BOD}_{5}$ results during the wet season. SSF Point 1-3 (Inlet/Thalia) and SF Point 4-7 (Brachiaria/Cyperus) presented 
better results for wet season $\mathrm{BOD}_{5}$ removal, with an average residual of $64 \% \pm 17 \%$ and $53 \% \pm 26 \%$, respectively. However, when looking at the complete CW System, Point 14-7 (Inlet/Brachiaria/Cyperus) presented better overall results with an average residual of $36 \% \pm 25 \%$.

Table 3-1: BOD $_{5}$ Wet Season Summary

\begin{tabular}{|c|c|c|c|c|c|}
\hline $\begin{array}{c}\text { Location } \\
\text { Description }\end{array}$ & $\begin{array}{c}\text { Monitoring } \\
\text { Point } \\
\text { Location }\end{array}$ & $\begin{array}{c}\text { Wet Season } \\
\text { BOD } 5 \text { Minimum } \\
\text { Concentration } \\
(\mathrm{mg} / \mathrm{L})\end{array}$ & $\begin{array}{c}\text { Wet Season } \\
\text { BOD }_{5} \\
\text { Maximum } \\
\text { Concentration } \\
\quad(\mathrm{mg} / \mathrm{L})\end{array}$ & $\begin{array}{c}\text { Wet Season BOD } \\
\text { Average } \\
\text { Concentration } \pm \\
\text { Standard } \\
\text { Deviation }(\mathrm{mg} / \mathrm{L})\end{array}$ & $\begin{array}{c}\text { Wet Season } \\
\text { BOD } 5 \text { Average } \\
\text { \% Residual } \pm \\
\text { Standard } \\
\text { Deviation }\end{array}$ \\
\hline Inlet & 1 & 32.8 & 80.0 & $54.8 \pm 16.3$ & - \\
\hline \multirow[t]{3}{*}{$\mathrm{SSF}$} & $1-2$ & 13.7 & 78.1 & $39.6 \pm 20.9$ & $80 \% \pm 62 \%$ \\
\hline & $1-3$ & 21.3 & 55.1 & $33.3 \pm 10.0$ & $64 \% \pm 17 \%$ \\
\hline & $1-4$ & 21.2 & 49.3 & $33.5 \pm 8.2$ & $65 \% \pm 19 \%$ \\
\hline \multirow[t]{3}{*}{$\mathrm{SF}$} & $2-5$ & 10.8 & 37.3 & $20.2 \pm 8.1$ & $60 \% \pm 23 \%$ \\
\hline & $3-6$ & 8.7 & 45.4 & $21.7 \pm 10.7$ & $64 \% \pm 19 \%$ \\
\hline & $4-7$ & 5.5 & 37.2 & $18.5 \pm 11.6$ & $53 \% \pm 26 \%$ \\
\hline \multirow{3}{*}{$\begin{array}{l}\text { Overall CW } \\
(\mathrm{SSF}+\mathrm{SF})\end{array}$} & $1-2-5$ & same as $2-5$ & same as $2-5$ & same as $2-5$ & $39 \% \pm 13 \%$ \\
\hline & $1-3-6$ & same as 3-6 & same as 3-6 & same as 3-6 & $42 \% \pm 20 \%$ \\
\hline & $1-4-7$ & same as 4-7 & same as 4-7 & same as 4-7 & $36 \% \pm 25 \%$ \\
\hline
\end{tabular}

The $\mathrm{BOD}_{5}$ average effluent concentrations ranged from 23.9 to $79.2 \mathrm{mg} / \mathrm{L}$ during the dry season (December to April), which is considered a much larger range compared to the wet season average range. Table 3-2 summarizes the $\mathrm{BOD}_{5}$ results during the dry season. SSF Point 1-4 (Inlet/Brachiaria) and SF Point 3-6 (Thalia/Thalia) presented better results for dry season $\mathrm{BOD}_{5}$ removal, with an average residual of $64 \% \pm 21 \%$ and $36 \% \pm 23 \%$, respectively. However, when looking at the complete CW System, Point 1-3-6 (Inlet/Thalia/Thalia) presented better overall results with an average residual of $33 \% \pm$ 
$22 \%$. Consistent with the achieved results, other studies results show that $\mathrm{BOD}_{5}$ residual can vary from $18 \%$ to $35 \%$, depending on the type of plant and climate conditions (Stefanakis \& Tsihrintzis, 2009).

Table 3-2: BOD $_{5}$ Dry Season Summary

\begin{tabular}{|c|c|c|c|c|c|}
\hline $\begin{array}{c}\text { Location } \\
\text { Description }\end{array}$ & $\begin{array}{c}\text { Monitoring } \\
\text { Point } \\
\text { Location }\end{array}$ & $\begin{array}{c}\text { Dry Season } \\
\text { BOD } 5 \text { Minimum } \\
\text { Concentration } \\
(\mathrm{mg} / \mathrm{L})\end{array}$ & $\begin{array}{c}\text { Dry Season } \\
\text { BOD } 5 \text { Maximum } \\
\text { Concentration } \\
(\mathrm{mg} / \mathrm{L})\end{array}$ & $\begin{array}{c}\text { Dry Season BOD } \\
\text { Average } \\
\text { Concentration } \pm \\
\text { Standard } \\
\text { Deviation }(\mathrm{mg} / \mathrm{L})\end{array}$ & $\begin{array}{c}\text { Dry Season } \\
\text { BOD } 5 \text { Average } \\
\text { \% Residual } \pm \\
\text { Standard } \\
\text { Deviation }\end{array}$ \\
\hline Inlet & 1 & 48.2 & 152.3 & $81.3 \pm 30.8$ & - \\
\hline \multirow[t]{3}{*}{ SSF } & $1-2$ & 46.8 & 92.3 & $62.8 \pm 14.6$ & $82 \% \pm 22 \%$ \\
\hline & $1-3$ & 39.2 & 167.4 & $76.0 \pm 40.2$ & $94 \% \pm 31 \%$ \\
\hline & $1-4$ & 36.4 & 80.7 & $48.9 \pm 14.2$ & $64 \% \pm 21 \%$ \\
\hline \multirow[t]{3}{*}{ SF } & $2-5$ & 7.7 & 520.0 & $79.2 \pm 178.2$ & $146 \% \pm 339 \%$ \\
\hline & $3-6$ & 11.0 & 44.4 & $23.4 \pm 13.6$ & $36 \% \pm 23 \%$ \\
\hline & $4-7$ & 9.9 & 73.0 & $28.9 \pm 22.4$ & $66 \% \pm 55 \%$ \\
\hline \multirow{3}{*}{$\begin{array}{c}\text { Overall CW } \\
(\mathrm{SSF}+\mathrm{SF})\end{array}$} & $1-2-5$ & same as $2-5$ & same as $2-5$ & same as $2-5$ & $98 \% \pm 213 \%$ \\
\hline & $1-3-6$ & same as 3-6 & same as 3-6 & same as 3-6 & $33 \% \pm 22 \% \%$ \\
\hline & $1-4-7$ & same as 4-7 & same as $4-7$ & same as 4-7 & $39 \% \pm 29 \%$ \\
\hline
\end{tabular}

\subsubsection{TSS}

TSS concentrations were consistently reduced by the CW System, as shown in Figure 3-2. Figure 3-2 shows TSS spikes during dry period that can be attributed to algae growth in the facultative lagoon. Algae growth was suppressed during the wet season. Seasonal results are summarized below. 


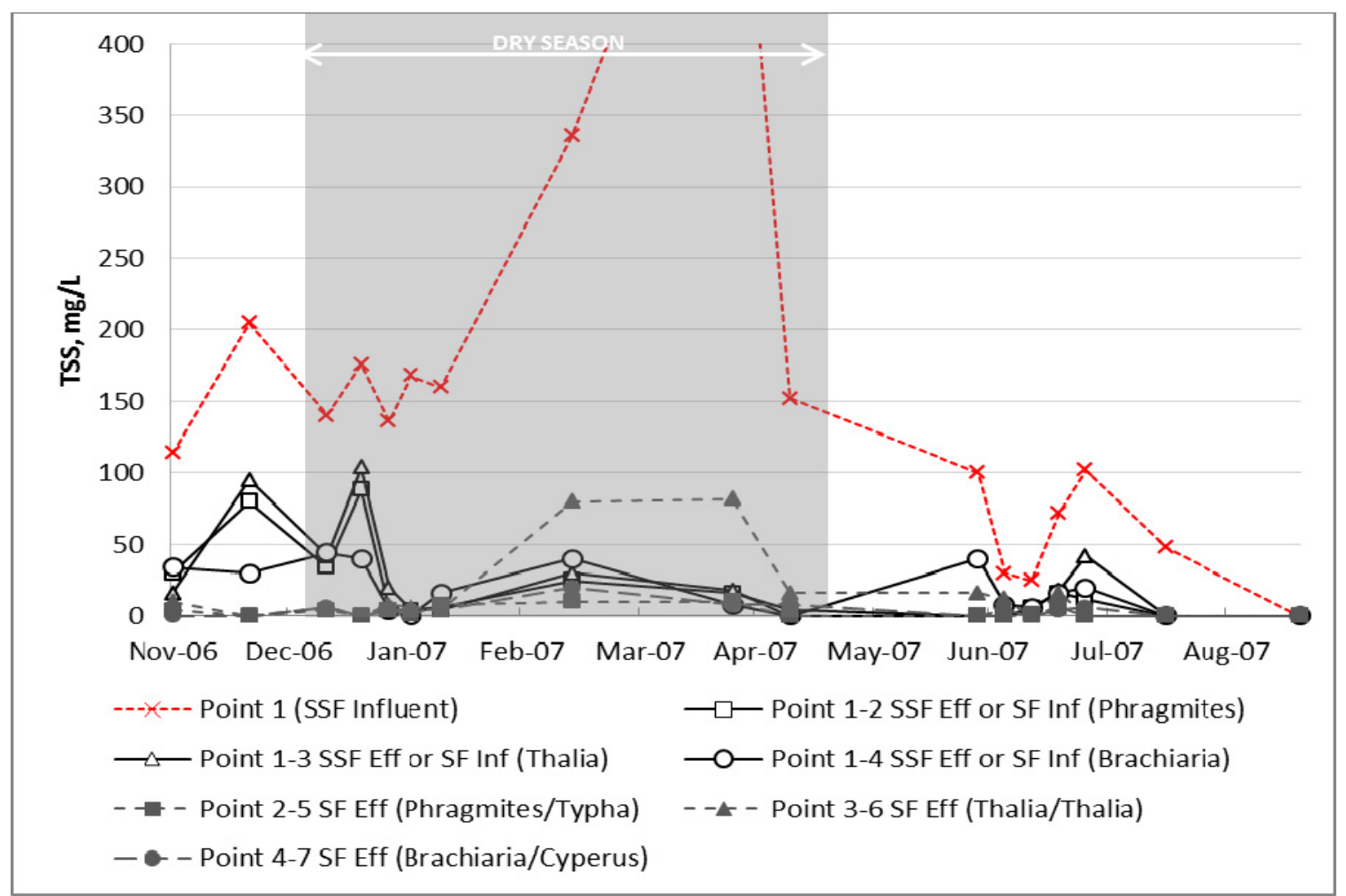

Figure 3-2: TSS Influent vs. Effluent Concentrations

Table 3-3 summarizes the TSS results during the wet season (May to November). SSF Point 1-2 (Inlet/Phragmites) and SF Point 2-5 (Phragmites/Typha) presented better results for wet season TSS removal, with an average residual of $11 \% \pm 12 \%$ and $8 \% \pm 14 \%$, respectively. Consistently with the individual cell results, when looking at the complete CW System, Point 1-2-5 (Inlet/Phragmites/Typha) presented better overall results with an average residual of $2 \% \pm 3 \%$. 
Table 3-3: TSS Wet Season Summary

\begin{tabular}{|c|c|c|c|c|c|}
\hline $\begin{array}{c}\text { Location } \\
\text { Description }\end{array}$ & $\begin{array}{l}\text { Monitoring } \\
\text { Point } \\
\text { Location }\end{array}$ & $\begin{array}{c}\text { Wet Season TSS } \\
\text { Minimum } \\
\text { Concentration } \\
(\mathrm{mg} / \mathrm{L})\end{array}$ & $\begin{array}{c}\text { Wet Season } \\
\text { TSS } \\
\text { Maximum } \\
\text { Concentration } \\
(\mathrm{mg} / \mathrm{L})\end{array}$ & $\begin{array}{c}\text { Wet Season TSS } \\
\text { Average } \\
\text { Concentration } \pm \\
\text { Standard } \\
\text { Deviation }(\mathrm{mg} / \mathrm{L})\end{array}$ & $\begin{array}{c}\text { Wet Season TSS } \\
\text { Average \% } \\
\text { Residual } \pm \\
\text { Standard } \\
\text { Deviation }\end{array}$ \\
\hline Inlet & 1 & 25.0 & 152.0 & $80.4 \pm 44.5$ & - \\
\hline \multirow[t]{3}{*}{$\mathrm{SSF}$} & $1-2$ & 0.0 & 30.0 & $8.5 \pm 10.5$ & $11 \% \pm 12 \%$ \\
\hline & $1-3$ & 0.0 & 42.0 & $10.0 \pm 14.9$ & $12 \% \pm 15 \%$ \\
\hline & $1-4$ & 0.0 & 40.0 & $15.5 \pm 15.1$ & $20 \% \pm 14 \%$ \\
\hline \multirow[t]{3}{*}{ SF } & $2-5$ & 0.0 & 6.0 & $1.4 \pm 2.3$ & $8 \% \pm 14 \%$ \\
\hline & $3-6$ & 0.0 & 16.0 & $8.8 \pm 7.6$ & $5,519 \% \pm 7,675 \%$ \\
\hline & $4-7$ & 0.0 & 40.0 & $2.8 \pm 3.2$ & $1,013 \% \pm 2,823 \%$ \\
\hline \multirow{3}{*}{$\begin{array}{c}\text { Overall CW } \\
(\mathrm{SSF}+\mathrm{SF})\end{array}$} & $1-2-5$ & same as $2-5$ & same as 2-5 & same as 2-5 & $2 \% \pm 3 \%$ \\
\hline & $1-3-6$ & same as 3-6 & same as 3-6 & same as 3-6 & $12 \% \pm 14 \%$ \\
\hline & $1-4-7$ & same as $4-7$ & same as 4-7 & same as $4-7$ & $4 \% \pm 5 \%$ \\
\hline
\end{tabular}

Table 3-4 summarizes the TSS results during the dry season (December to April). SSF Point 1-4 (Inlet/Brachiaria) and SF Point 2-5 (Phragmites/Typha) presented better results for dry season TSS removal, with an average residual of $12 \% \pm 11 \%$ and $46 \% \pm 49 \%$, respectively. However, when looking at the complete CW System, Point 1-2-5 (Inlet/Phragmites/Typha) presented better overall results with an average residual of $2 \%$ $\pm 2 \%$. Consistent with the achieved results, other studies results show that TSS residual can vary from $0 \%$ to $40 \%$, depending on the type of flow pattern, plant and climate conditions (Pedescoll, et al., 2011). 
Table 3-4: TSS Dry Season Summary

\begin{tabular}{|c|c|c|c|c|c|}
\hline $\begin{array}{c}\text { Location } \\
\text { Description }\end{array}$ & $\begin{array}{c}\text { Monitoring } \\
\text { Point } \\
\text { Location }\end{array}$ & $\begin{array}{c}\text { Dry Season TSS } \\
\text { Minimum } \\
\text { Concentration } \\
(\mathrm{mg} / \mathrm{L})\end{array}$ & $\begin{array}{l}\text { Dry Season TSS } \\
\text { Maximum } \\
\text { Concentration } \\
(\mathrm{mg} / \mathrm{L})\end{array}$ & $\begin{array}{c}\text { Dry Season TSS } \\
\text { Average } \\
\text { Concentration } \pm \\
\text { Standard } \\
\text { Deviation }(\mathrm{mg} / \mathrm{L})\end{array}$ & $\begin{array}{c}\text { Dry Season } \\
\text { TSS Average } \\
\text { \% Residual } \pm \\
\text { Standard } \\
\text { Deviation }\end{array}$ \\
\hline Inlet & 1 & 136.0 & 630.0 & $243.8 \pm 168.5$ & - \\
\hline \multirow[t]{3}{*}{ SSF } & $1-2$ & 4.0 & 88.0 & $32.1 \pm 33.8$ & $17 \% \pm 19 \%$ \\
\hline & $1-3$ & 4.0 & 104.0 & $39.6 \pm 39.1$ & $21 \% \pm 22 \%$ \\
\hline & $1-4$ & 0.0 & 44.0 & $22.8 \pm 17.9$ & $12 \% \pm 11 \%$ \\
\hline \multirow[t]{3}{*}{$\mathrm{SF}$} & $2-5$ & 0.0 & 10.0 & $4.6 \pm 4.3$ & $46 \% \pm 49 \%$ \\
\hline & $3-6$ & 0.0 & 82.0 & $23.3 \pm 35.8$ & $128 \% \pm 161 \%$ \\
\hline & $4-7$ & 0.0 & 20.0 & $5.8 \pm 6.4$ & $536 \% \pm 1400 \%$ \\
\hline \multirow{3}{*}{$\begin{array}{l}\text { Overall CW } \\
(\mathrm{SSF}+\mathrm{SF})\end{array}$} & $1-2-5$ & same as $2-5$ & same as $2-5$ & same as $2-5$ & $2 \% \pm 2 \%$ \\
\hline & $1-3-6$ & same as 3-6 & same as 3-6 & same as 3-6 & $7 \% \pm 8 \%$ \\
\hline & $1-4-7$ & same as 4-7 & same as 4-7 & same as 4-7 & $4 \% \pm 5 \%$ \\
\hline
\end{tabular}

\subsubsection{COD}

COD concentrations were mostly reduced by the CW System as shown in Figure 3-3.

Seasonal results are summarized below. 


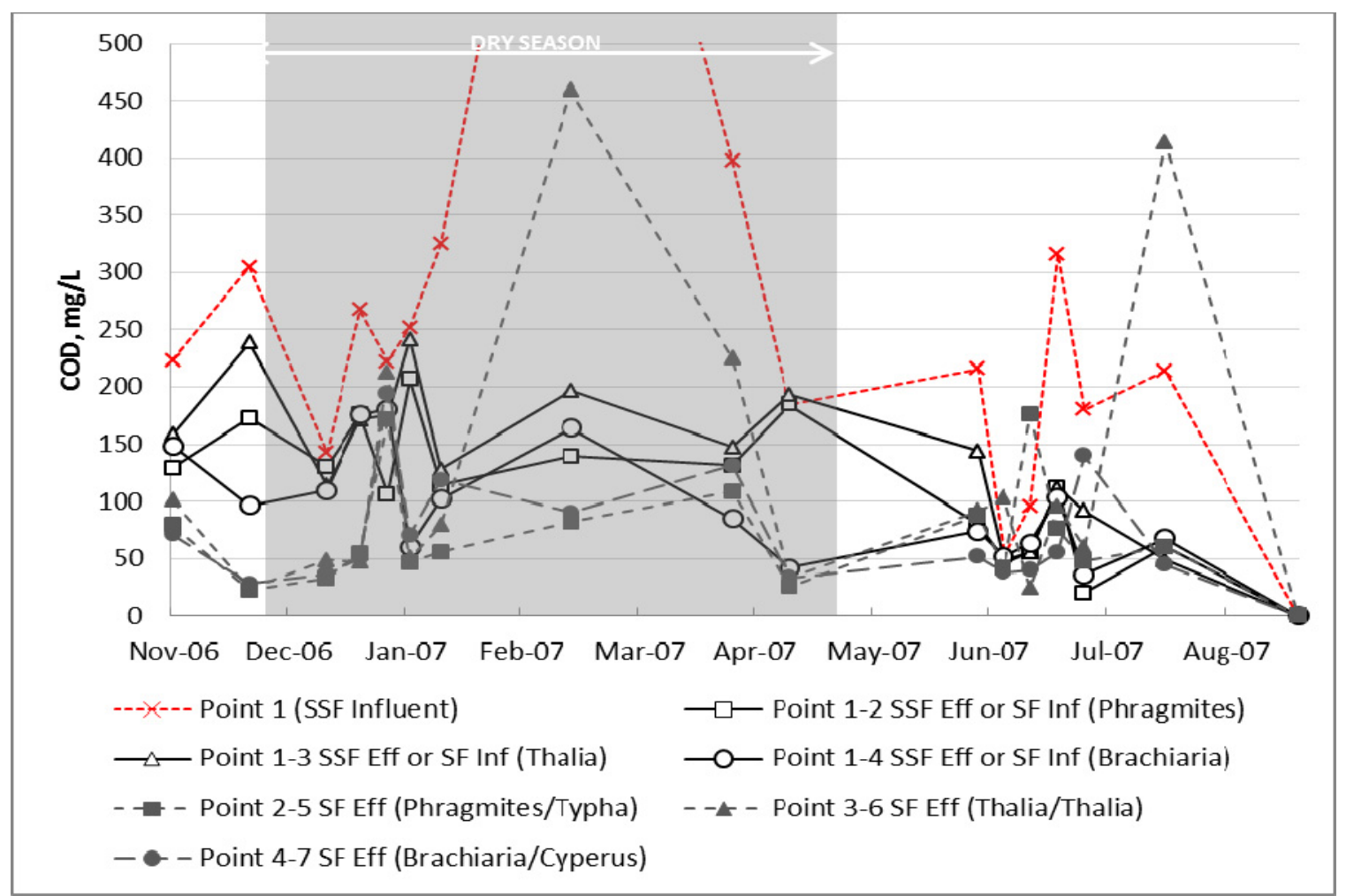

Figure 3-3: COD Influent vs. Effluent Concentrations

Table 3-5 summarizes the COD results during the wet season (May to November). SSF Point 1-4 (Inlet/Brachiaria) and SF Point 4-7 (Brachiaria/Cyperus) presented better results for wet season COD removal, with an average residual of $47 \% \pm 28 \%$ and $105 \% \pm$ $115 \%$, respectively. Consistently with the individual cell results, when looking at the complete CW System, Point 1-4-7 (Inlet/Brachiaria/Cyperus) presented better overall results with an average residual of $38 \% \pm 24 \%$. 
Table 3-5: COD Wet Season Summary

\begin{tabular}{|c|c|c|c|c|c|}
\hline $\begin{array}{c}\text { Location } \\
\text { Description }\end{array}$ & $\begin{array}{c}\text { Monitoring } \\
\text { Point } \\
\text { Location }\end{array}$ & $\begin{array}{c}\text { Wet Season COD } \\
\text { Minimum } \\
\text { Concentration } \\
(\mathrm{mg} / \mathrm{L})\end{array}$ & $\begin{array}{c}\text { Wet Season COD } \\
\text { Maximum } \\
\text { Concentration } \\
(\mathrm{mg} / \mathrm{L})\end{array}$ & $\begin{array}{c}\text { Wet Season COD } \\
\text { Average } \\
\text { Concentration } \pm \\
\text { Standard } \\
\text { Deviation }(\mathrm{mg} / \mathrm{L})\end{array}$ & $\begin{array}{c}\text { Wet Season } \\
\text { COD Average } \\
\% \text { Residual } \pm \\
\text { Standard } \\
\text { Deviation }\end{array}$ \\
\hline Inlet & 1 & 51.6 & 316.0 & $185.3 \pm 81.2$ & - \\
\hline \multirow[t]{3}{*}{ SSF } & $1-2$ & 20.0 & 184.9 & $86.2 \pm 53.1$ & $53 \% \pm 31 \%$ \\
\hline & $1-3$ & 45.6 & 193.3 & $106.3 \pm 55.4$ & $62 \% \pm 27 \%$ \\
\hline & $1-4$ & 36.0 & 148.8 & $73.5 \pm 37.0$ & $47 \% \pm 28 \%$ \\
\hline \multirow[t]{3}{*}{ SF } & $2-5$ & 25.2 & 176.0 & $74.3 \pm 46.2$ & $124 \% \pm 101 \%$ \\
\hline & $3-6$ & 24.0 & 413.5 & $115.4 \pm 124.4$ & $176 \% \pm 278 \%$ \\
\hline & $4-7$ & 33.6 & 140.0 & $59.5 \pm 34.7$ & $105 \% \pm 115 \%$ \\
\hline \multirow{3}{*}{$\begin{array}{l}\text { Overall CW } \\
(\mathrm{SSF}+\mathrm{SF})\end{array}$} & $1-2-5$ & same as $2-5$ & same as $2-5$ & same as $2-5$ & $54 \% \pm 56 \%$ \\
\hline & $1-3-6$ & same as 3-6 & same as 3-6 & same as 3-6 & $73 \% \pm 76 \%$ \\
\hline & $1-4-7$ & same as $4-7$ & same as 4-7 & same as 4-7 & $38 \% \pm 24 \%$ \\
\hline
\end{tabular}

Table 3-6 summarizes the COD results during the dry season (December to April). SSF Point 1-4 (Inlet/Brachiaria) and SF Point 2-5 (Phragmites/Typha) presented better results for dry season COD removal, with an average residual of $44 \% \pm 26 \%$ and $55 \% \pm 48 \%$, respectively. However, when looking at the complete CW System, Point 1-2-5 (Inlet/Phragmites/Typha) presented better overall results with an average residual of $25 \%$ $\pm 22 \%$. Consistent with the achieved results, other studies results show that COD residual can vary from $22 \%$ to $39 \%$, depending on the type of plant and climate conditions (Stefanakis \& Tsihrintzis, 2009).

Even though Point 1-2-5 (Inlet/Phragmites/Typha) and Point 1-4-7

(Inlet/Brachiaria/Cyperus) produced better results for the dry and wet seasons, respectively; both cells achieved acceptable effluent concentrations in accordance with local regulations requirements. 
Table 3-6: COD Dry Season Summary

\begin{tabular}{|c|c|c|c|c|c|}
\hline $\begin{array}{c}\text { Location } \\
\text { Description }\end{array}$ & $\begin{array}{c}\text { Monitoring } \\
\text { Point } \\
\text { Location }\end{array}$ & $\begin{array}{c}\text { Dry Season } \\
\text { COD Minimum } \\
\text { Concentration } \\
(\mathrm{mg} / \mathrm{L})\end{array}$ & $\begin{array}{c}\text { Dry Season } \\
\text { COD Maximum } \\
\text { Concentration } \\
(\mathrm{mg} / \mathrm{L})\end{array}$ & $\begin{array}{c}\text { Dry Season COD } \\
\text { Average } \\
\text { Concentration } \pm \\
\text { Standard Deviation } \\
(\mathrm{mg} / \mathrm{L})\end{array}$ & $\begin{array}{c}\text { Dry Season } \\
\text { COD Average } \\
\% \text { Residual } \pm \\
\text { Standard } \\
\text { Deviation }\end{array}$ \\
\hline Inlet & 1 & 142.3 & 922.1 & $354.2 \pm 241.4$ & - \\
\hline \multirow[t]{3}{*}{ SSF } & $1-2$ & 106.5 & 206.7 & $147.1 \pm 34.5$ & $53 \% \pm 26 \%$ \\
\hline & $1-3$ & 113.8 & 241.9 & $176.8 \pm 47.4$ & $62 \% \pm 26 \%$ \\
\hline & $1-4$ & 60.5 & 180.6 & $121.8 \pm 45.4$ & $44 \% \pm 26 \%$ \\
\hline \multirow[t]{3}{*}{ SF } & $2-5$ & 22.4 & 171.3 & $71.7 \pm 48.6$ & $55 \% \pm 48 \%$ \\
\hline & $3-6$ & 24.4 & 459.0 & $143.3 \pm 149.3$ & $84 \% \pm 79 \%$ \\
\hline & $4-7$ & 28.5 & 194.4 & $90.2 \pm 56.1$ & $80 \% \pm 49 \%$ \\
\hline \multirow{3}{*}{$\begin{array}{c}\text { Overall CW } \\
(\mathrm{SSF}+\mathrm{SF})\end{array}$} & $1-2-5$ & same as $2-5$ & same as $2-5$ & same as 2-5 & $25 \% \pm 22 \%$ \\
\hline & $1-3-6$ & same as 3-6 & same as 3-6 & same as 3-6 & $38 \% \pm 28 \%$ \\
\hline & $1-4-7$ & same as $4-7$ & same as 4-7 & same as 4-7 & $31 \% \pm 25 \%$ \\
\hline
\end{tabular}

\subsubsection{Fecal Coliform}

Fecal coliform concentrations were reduced by the CW System as expected from other studies results, with residuals in the upper 10\% (Lazareva \& Pichler, 2010). Seasonal results are summarized below and illustrated in Figure 3-4. . 


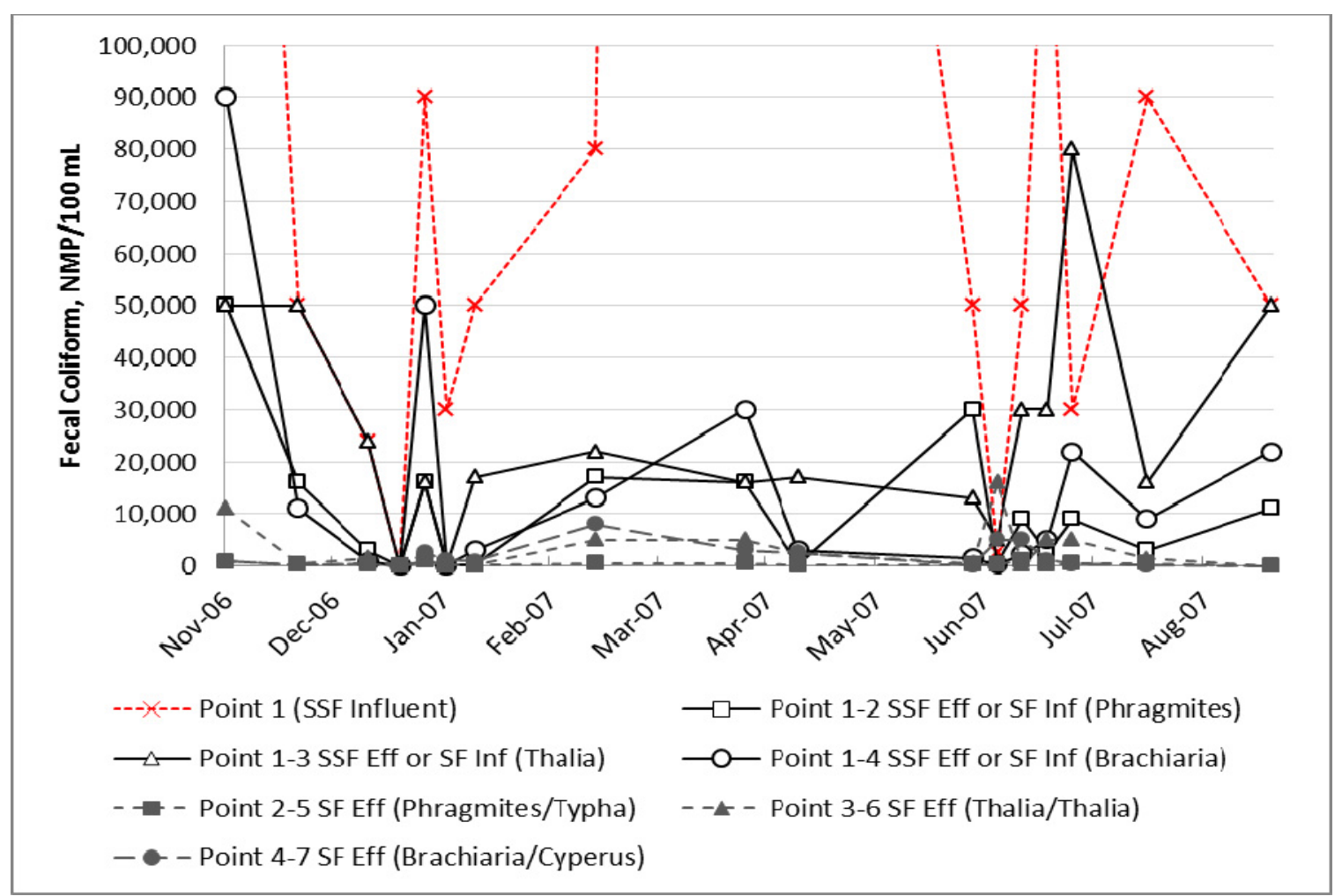

Figure 3-4: Fecal Coliform Influent vs. Effluent Concentrations

Table 3-7 summarizes the Fecal Coliform results during the wet season (May to November). SSF Point 1-2 (Inlet/Phragmites) and SF Point 2-5 (Phragmites/Typha) presented better results for wet season Fecal Coliforms removal, with an average residual of $18 \% \pm 19 \%$ and $21 \% \pm 27 \%$, respectively. Consistently with the individual cell results, when looking at the complete CW System, Point 1-2-5 (Inlet/Phragmites/Typha) presented better overall results with an average residual of $2 \% \pm 2 \%$. Higher residuals values during the wet season can be attributed to increased disturbance of soil particles during heavy rains and greater summer humidity conducive to Fecal Coliforms survival (Katsenovich, et al., 2009). 
Table 3-7: Fecal Coliform Wet Season Summary

\begin{tabular}{|c|c|c|c|c|c|}
\hline $\begin{array}{c}\text { Location } \\
\text { Description }\end{array}$ & $\begin{array}{c}\text { Monitoring } \\
\text { Point } \\
\text { Location }\end{array}$ & $\begin{array}{c}\text { Wet Season FC } \\
\text { Minimum } \\
\text { Concentration } \\
\text { (NMP/100mL) }\end{array}$ & $\begin{array}{c}\text { Wet Season FC } \\
\text { Maximum } \\
\text { Concentration } \\
\text { (NMP/100mL }\end{array}$ & $\begin{array}{c}\text { Wet Season FC } \\
\text { Average } \\
\text { Concentration } \pm \\
\text { Standard Deviation } \\
\text { (NMP/100mL) }\end{array}$ & $\begin{array}{c}\text { Wet Season } \\
\text { FC Average } \\
\text { \% Residual } \\
\pm \text { Standard } \\
\text { Deviation }\end{array}$ \\
\hline Inlet & 1 & 2,400 & 300,000 & $112,489 \pm 113,039$ & - \\
\hline \multirow[t]{3}{*}{ SSF } & 1-2 & 240 & 50,000 & $12,764 \pm 16,701$ & $18 \% \pm 19 \%$ \\
\hline & $1-3$ & 5,000 & 80,000 & $32,333 \pm 23,796$ & $80 \% \pm 95 \%$ \\
\hline & $1-4$ & 240 & 90,000 & $17,204 \pm 28,561$ & $20 \% \pm 25 \%$ \\
\hline \multirow[t]{3}{*}{ SF } & $2-5$ & 130 & 1,100 & $448 \pm 353$ & $21 \% \pm 27 \%$ \\
\hline & $3-6$ & 0 & 16,000 & $4,597 \pm 5,529$ & $44 \% \pm 104 \%$ \\
\hline & $4-7$ & 0 & 5,000 & $1,646 \pm 2,042$ & $\begin{array}{c}269 \% \pm \\
684 \%\end{array}$ \\
\hline \multirow{3}{*}{$\begin{array}{c}\text { Overall CW } \\
(\mathrm{SSF}+\mathrm{SF})\end{array}$} & $1-2-5$ & same as $2-5$ & same as 2-5 & same as $2-5$ & $2 \% \pm 2 \%$ \\
\hline & $1-3-6$ & same as 3-6 & same as 3-6 & same as 3-6 & $77 \% \pm 221 \%$ \\
\hline & $1-4-7$ & same as 4-7 & same as $4-7$ & same as $4-7$ & $25 \% \pm 69 \%$ \\
\hline
\end{tabular}

Table 3-8 summarizes the Fecal Coliform results during the dry season (December to April). SSF Point 1-2 (Inlet/Phragmites) and SF Point 2-5 (Phragmites/Typha) presented better results for dry season Fecal Coliforms removal, with an average residual of $12 \% \pm$ $12 \%$ and $6 \% \pm 4 \%$, respectively. Consistently with the individual cell results, when looking at the complete CW System, Point 1-2-5 (Inlet/Phragmites/Typha) presented better overall results with an average residual of $0 \% \pm 1 \%$. 
Table 3-8: Fecal Coliform Dry Season Summary

\begin{tabular}{|c|c|c|c|c|c|}
\hline $\begin{array}{c}\text { Location } \\
\text { Description }\end{array}$ & $\begin{array}{c}\text { Monitoring } \\
\text { Point } \\
\text { Location }\end{array}$ & $\begin{array}{c}\text { Dry Season FC } \\
\text { Minimum } \\
\text { Concentration } \\
(\mathrm{mg} / \mathrm{L})\end{array}$ & $\begin{array}{c}\text { Dry Season FC } \\
\text { Maximum } \\
\text { Concentration } \\
(\mathrm{mg} / \mathrm{L})\end{array}$ & $\begin{array}{c}\text { Dry Season FC } \\
\text { Average } \\
\text { Concentration } \pm \\
\text { Standard } \\
\text { Deviation }(\mathbf{m g} / \mathbf{L})\end{array}$ & $\begin{array}{c}\text { Dry Season } \\
\text { FC Average } \\
\% \text { Residual } \pm \\
\text { Standard } \\
\text { Deviation }\end{array}$ \\
\hline Inlet & 1 & 24,000 & $1,600,000$ & $274,857 \pm 584,829$ & - \\
\hline \multirow[t]{3}{*}{ SSF } & $1-2$ & 110 & 17,000 & $9,749 \pm 8,173$ & $12 \% \pm 12 \%$ \\
\hline & $1-3$ & 70 & 50,000 & $20,724 \pm 15,025$ & $40 \% \pm 43 \%$ \\
\hline & $1-4$ & 50 & 50,000 & $15,407 \pm 18,430$ & $15 \% \pm 20 \%$ \\
\hline \multirow[t]{3}{*}{ SF } & $2-5$ & 13 & 900 & $338 \pm 322$ & $6 \% \pm 4 \%$ \\
\hline & $3-6$ & 8 & 5,000 & $1,833 \pm 2,214$ & $58 \% \pm 126 \%$ \\
\hline & $4-7$ & 220 & 8,000 & $2,260 \pm 2,738$ & $335 \% \pm 823 \%$ \\
\hline \multirow{3}{*}{$\begin{array}{c}\text { Overall CW } \\
(\mathrm{SSF}+\mathrm{SF})\end{array}$} & $1-2-5$ & same as $2-5$ & same as $2-5$ & same as 2-5 & $0 \% \pm 1 \%$ \\
\hline & $1-3-6$ & same as 3-6 & same as 3-6 & same as 3-6 & $2 \% \pm 3 \%$ \\
\hline & $1-4-7$ & same as $4-7$ & same as $4-7$ & same as $4-7$ & $3 \% \pm 3 \%$ \\
\hline
\end{tabular}

\subsubsection{Oil \& Grease}

Oil and Grease concentrations were reduced by the CW System, with some exceptions, as shown in Figure 3-5. Seasonal results are summarized below. 


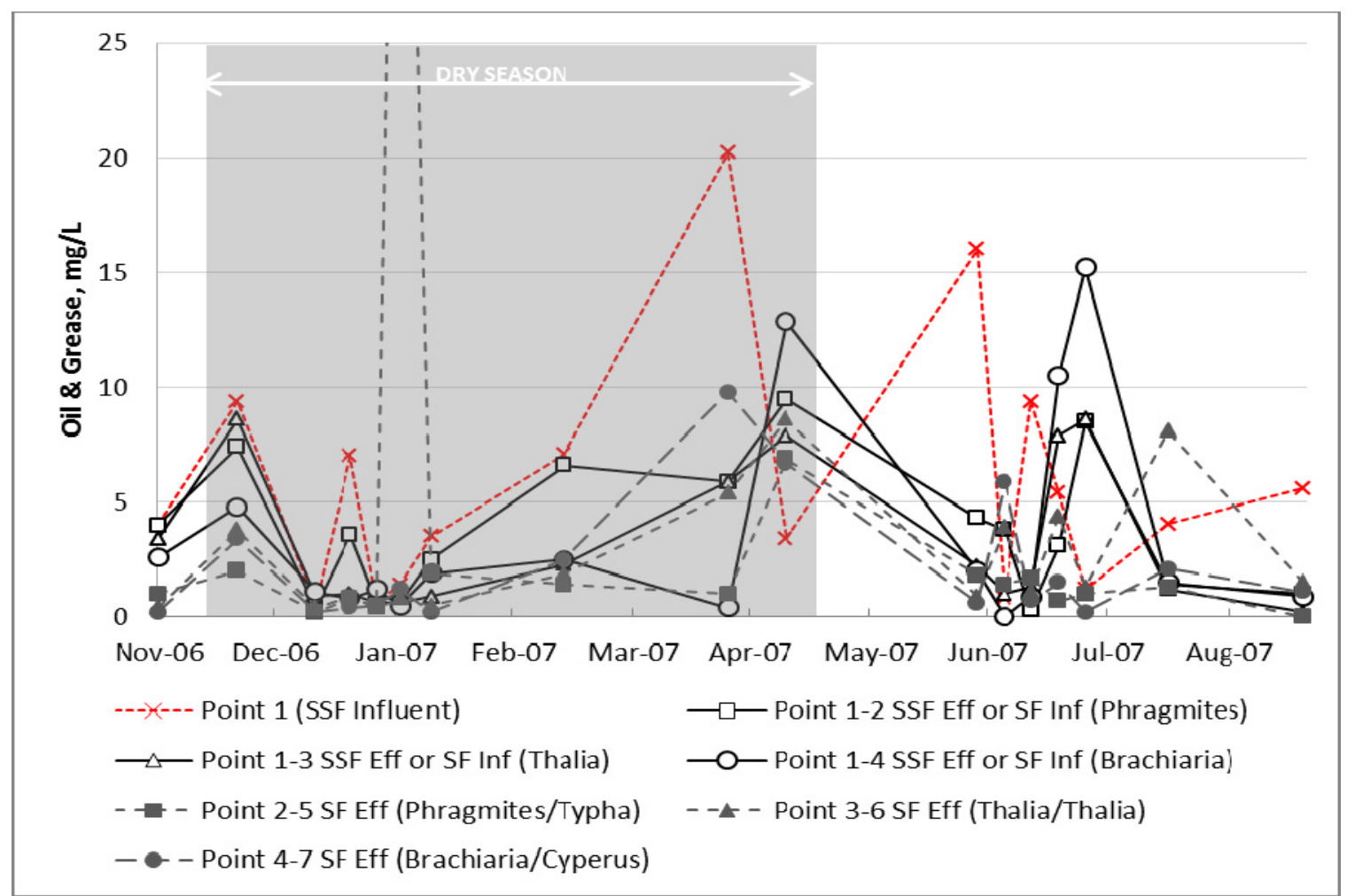

Figure 3-5: Oil \& Grease Influent vs. Effluent Concentrations

Table 3-9 summarizes the Oil \& Grease results during the wet season (May to November). The SSF CW did not successfully remove Oil \& Grease, with its best performance at the Point 1-3 (Inlet/Thalia) still having higher concentration than the inlet concentration $(152 \% \pm 225 \%$ residual). Point 4-7 (Brachiaria/Cyperus) presented better results for wet season Oil \& Grease removal, with an average residual of $56 \% \pm 53 \%$. Consistently with the individual cell results, when looking at the complete CW System, Point 1-4-7 (Inlet/Brachiaria/Cyperus) presented better overall results with an average residual of $41 \% \pm 65 \%$. 
Table 3-9: Oil \& Grease Wet Season Summary

\begin{tabular}{|c|c|c|c|c|c|}
\hline $\begin{array}{c}\text { Location } \\
\text { Description }\end{array}$ & $\begin{array}{c}\text { Monitoring } \\
\text { Point } \\
\text { Location }\end{array}$ & $\begin{array}{c}\text { Wet Season } \\
\text { O\&G Minimum } \\
\text { Concentration } \\
(\mathrm{mg} / \mathrm{L})\end{array}$ & $\begin{array}{c}\text { Wet Season } \\
\text { O\&G } \\
\text { Maximum } \\
\text { Concentration } \\
(\mathrm{mg} / \mathrm{L})\end{array}$ & $\begin{array}{c}\text { Wet Season O\&G } \\
\text { Average } \\
\text { Concentration } \pm \\
\text { Standard } \\
\text { Deviation }(\mathrm{mg} / \mathrm{L})\end{array}$ & $\begin{array}{c}\text { Wet Season } \\
\text { O\&G Average } \\
\text { \% Residual } \pm \\
\text { Standard } \\
\text { Deviation }\end{array}$ \\
\hline Inlet & 1 & 0.8 & 16.0 & $5.5 \pm 4.7$ & - \\
\hline \multirow[t]{3}{*}{$\mathrm{SSF}$} & $1-2$ & 0.3 & 9.5 & $4.0 \pm 3.2$ & $189 \% \pm 250 \%$ \\
\hline & 1-3 & 0.2 & 8.6 & $3.7 \pm 3.4$ & $152 \% \pm 225 \%$ \\
\hline & $1-4$ & 0.0 & 15.2 & $5.2 \pm 5.9$ & $220 \% \pm 412 \%$ \\
\hline \multirow[t]{3}{*}{ SF } & $2-5$ & 0.0 & 6.9 & $1.8 \pm 2.0$ & $97 \% \pm 179 \%$ \\
\hline & $3-6$ & 0.4 & 8.6 & $3.4 \pm 3.1$ & $237 \% \pm 294 \%$ \\
\hline & $4-7$ & 0.2 & 6.7 & $2.1 \pm 2.5$ & $56 \% \pm 53 \%$ \\
\hline \multirow{3}{*}{$\begin{array}{l}\text { Overall CW } \\
(\mathrm{SSF}+\mathrm{SF})\end{array}$} & $1-2-5$ & same as $2-5$ & same as $2-5$ & same as $2-5$ & $62 \% \pm 76 \%$ \\
\hline & $1-3-6$ & same as 3-6 & same as 3-6 & same as 3-6 & $132 \% \pm 160 \%$ \\
\hline & $1-4-7$ & same as $4-7$ & same as 4-7 & same as 4-7 & $41 \% \pm 65 \%$ \\
\hline
\end{tabular}

Table 3-10 summarizes the Oil \& Grease results during the dry season (December to April). SSF Point 1-3 (Inlet/Thalia) and SF Point 3-6 (Thalia/Thalia) presented better results for dry season Oil \& Grease removal, with an average residual of $64 \% \pm 56 \%$ and $76 \% \pm 30 \%$, respectively. Consistently with the individual cell results, when looking at the complete CW System, Point 1-3-6 (Inlet/Thalia/Thalia) presented better overall results with an average residual of $41 \% \pm 25 \%$.

Table 3-10: Oil \& Grease Dry Season Summary

\begin{tabular}{|c|c|c|c|c|c|}
\hline $\begin{array}{c}\text { Location } \\
\text { Description }\end{array}$ & $\begin{array}{l}\text { Monitoring } \\
\text { Point } \\
\text { Location }\end{array}$ & $\begin{array}{c}\text { Dry Season O\&G } \\
\text { Minimum } \\
\text { Concentration } \\
(\mathbf{m g} / \mathrm{L})\end{array}$ & $\begin{array}{c}\text { Dry Season } \\
\text { O\&G } \\
\text { Maximum } \\
\text { Concentration } \\
(\mathrm{mg} / \mathrm{L})\end{array}$ & $\begin{array}{c}\text { Dry Season } \\
\text { O\&G Average } \\
\text { Concentration } \pm \\
\text { Standard } \\
\text { Deviation }(\mathrm{mg} / \mathrm{L})\end{array}$ & $\begin{array}{c}\text { Dry Season O\&G } \\
\text { Average \% } \\
\text { Residual } \pm \\
\text { Standard } \\
\text { Deviation }\end{array}$ \\
\hline Inlet & 1 & 0.5 & 20.2 & $6.2 \pm 6.6$ & - \\
\hline \multirow[t]{3}{*}{ SSF } & $1-2$ & 0.4 & 7.4 & $3.5 \pm 2.9$ & $68 \% \pm 25 \%$ \\
\hline & $1-3$ & 0.6 & 8.6 & $2.6 \pm 3.0$ & $64 \% \pm 56 \%$ \\
\hline & $1-4$ & 0.4 & 4.8 & $1.7 \pm 1.5$ & $76 \% \pm 85 \%$ \\
\hline \multirow[t]{2}{*}{ SF } & $2-5$ & 0.2 & 55.4 & $7.9 \pm 19.2$ & $1192 \% \pm 3252 \%$ \\
\hline & $3-6$ & 0.4 & 5.4 & $1.8 \pm 1.9$ & $76 \% \pm 30 \%$ \\
\hline
\end{tabular}




\begin{tabular}{|c|c|c|c|c|c|}
\hline $\begin{array}{c}\text { Location } \\
\text { Description }\end{array}$ & $\begin{array}{c}\text { Monitoring } \\
\text { Point } \\
\text { Location }\end{array}$ & $\begin{array}{c}\text { Dry Season O\&G } \\
\text { Minimum } \\
\text { Concentration } \\
(\mathrm{mg} / \mathrm{L})\end{array}$ & $\begin{array}{c}\text { Dry Season } \\
\text { O\&G } \\
\text { Maximum } \\
\text { Concentration } \\
(\mathrm{mg} / \mathrm{L})\end{array}$ & $\begin{array}{c}\text { Dry Season } \\
\text { O\&G Average } \\
\text { Concentration } \pm \\
\text { Standard } \\
\text { Deviation }(\mathrm{mg} / \mathrm{L})\end{array}$ & $\begin{array}{c}\text { Dry Season O\&G } \\
\text { Average \% } \\
\text { Residual } \pm \\
\text { Standard } \\
\text { Deviation }\end{array}$ \\
\hline & $4-7$ & 0.2 & 9.8 & $2.3 \pm 3.3$ & $373 \% \pm 843 \%$ \\
\hline \multirow{3}{*}{$\begin{array}{c}\text { Overall CW } \\
(\mathrm{SSF}+\mathrm{SF})\end{array}$} & $1-2-5$ & same as $2-5$ & same as $2-5$ & same as $2-5$ & $524 \% \pm 1388 \%$ \\
\hline & $1-3-6$ & same as 3-6 & same as 3-6 & same as 3-6 & $41 \% \pm 25 \%$ \\
\hline & $1-4-7$ & same as 4-7 & same as 4-7 & same as 4-7 & $43 \% \pm 30 \%$ \\
\hline
\end{tabular}

\subsubsection{Total Nitrogen}

Total Nitrogen concentrations were reduced by the CW System, with a few exceptions, as shown in Figure 3-5. Seasonal results are summarized below.

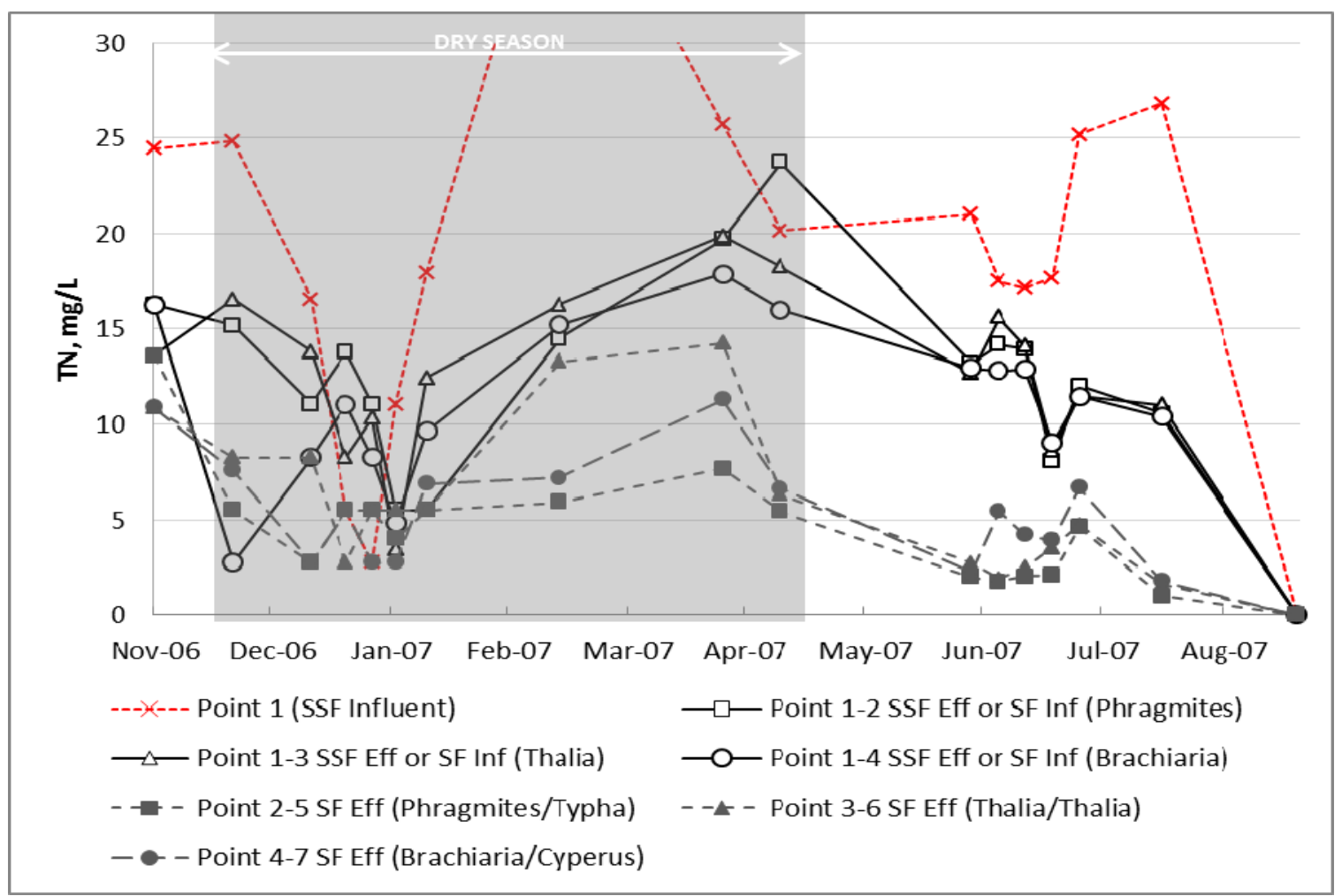

Figure 3-6: Total Nitrogen Influent vs. Effluent Concentrations 
Table 3-11 summarizes the Total Nitrogen results during the wet season (May to November). SSF Point 1-4 (Inlet/Brachiaria) and SF Point 2-5 (Phragmites/Typha) presented better results for wet season Total Nitrogen removal, with an average residual of $61 \% \pm 15 \%$ and $28 \% \pm 24 \%$, respectively. However, when looking at the complete CW System, Point 1-2-5 (Inlet/Phragmites/Typha) presented better overall results with an average residual of $19 \% \pm 16 \%$.

Table 3-11: Total Nitrogen Wet Season Summary

\begin{tabular}{|c|c|c|c|c|c|}
\hline $\begin{array}{c}\text { Location } \\
\text { Description }\end{array}$ & $\begin{array}{c}\text { Monitoring } \\
\text { Point } \\
\text { Location }\end{array}$ & $\begin{array}{l}\text { Wet Season TN } \\
\text { Minimum } \\
\text { Concentration } \\
(\mathrm{mg} / \mathrm{L})\end{array}$ & $\begin{array}{c}\text { Wet Season TN } \\
\text { Maximum } \\
\text { Concentration } \\
(\mathrm{mg} / \mathrm{L})\end{array}$ & $\begin{array}{c}\text { Wet Season TN } \\
\text { Average } \\
\text { Concentration } \pm \\
\text { Standard } \\
\text { Deviation }(\mathrm{mg} / \mathrm{L})\end{array}$ & $\begin{array}{c}\text { Wet Season } \\
\text { TN Average } \\
\text { \% Residual } \pm \\
\text { Standard } \\
\text { Deviation }\end{array}$ \\
\hline Inlet & 1 & 17.1 & 26.8 & $21.1 \pm 3.8$ & - \\
\hline \multirow[t]{3}{*}{ SSF } & $1-2$ & 8.1 & 23.8 & $14.0 \pm 4.7$ & $68 \% \pm 26 \%$ \\
\hline & $1-3$ & 8.7 & 18.2 & $13.2 \pm 2.9$ & $64 \% \pm 20 \%$ \\
\hline & $1-4$ & 9.0 & 16.3 & $12.7 \pm 2.5$ & $61 \% \pm 15 \%$ \\
\hline \multirow[t]{3}{*}{ SF } & $2-5$ & 1.0 & 13.6 & $4.1 \pm 4.1$ & $28 \% \pm 24 \%$ \\
\hline & $3-6$ & 1.6 & 10.9 & $4.3 \pm 3.1$ & $33 \% \pm 22 \%$ \\
\hline & $4-7$ & 1.8 & 10.9 & $5.2 \pm 2.9$ & $40 \% \pm 17 \%$ \\
\hline \multirow{3}{*}{$\begin{array}{l}\text { Overall CW } \\
(\mathrm{SSF}+\mathrm{SF})\end{array}$} & $1-2-5$ & same as 2-5 & same as 2-5 & same as $2-5$ & $19 \% \pm 16 \%$ \\
\hline & $1-3-6$ & same as 3-6 & same as 3-6 & same as 3-6 & $20 \% \pm 12 \%$ \\
\hline & $1-4-7$ & same as 4-7 & same as 4-7 & same as $4-7$ & $25 \% \pm 12 \%$ \\
\hline
\end{tabular}

Table 3-12 summarizes the Total Nitrogen results during the dry season (December to April). The SSF CW did not successfully remove Total Nitrogen, with its best performance at the Point 1-4 (Inlet/Brachiaria) still having a high residual $(96 \% \pm 100 \%$ residual). Point 2-5 (Phragmites/Typha) presented better results for dry season Total Nitrogen removal, with an average residual of $50 \% \pm 24 \%$. However, when looking at 
the complete CW System, Point 1-4-7 (Inlet/Thalia/Thalia) presented better overall results with an average residual of $46 \% \pm 34 \%$.

The dry season had higher Total Nitrogen concentrations than the wet season, which could be associated to longer retention time and vegetation decay to during the dry season. A review of other studies indicates that Total Nitrogen residuals through SSF CWs typically range between $45 \%$ and 60\% (Vymazal, 2007) (Tuncsiper, 2009), which is considered similar results to the achieved in this study. Other studies results for Total Nitrogen residual through a combination of SSF and SF CWs have not been reported.

Table 3-12: Total Nitrogen Dry Season Summary

\begin{tabular}{|c|c|c|c|c|c|}
\hline $\begin{array}{c}\text { Location } \\
\text { Description }\end{array}$ & $\begin{array}{l}\text { Monitoring } \\
\text { Point } \\
\text { Location }\end{array}$ & $\begin{array}{c}\text { Dry Season TN } \\
\text { Minimum } \\
\text { Concentration } \\
(\mathrm{mg} / \mathrm{L})\end{array}$ & $\begin{array}{c}\text { Dry Season TN } \\
\text { Maximum } \\
\text { Concentration } \\
(\mathrm{mg} / \mathrm{L})\end{array}$ & $\begin{array}{c}\text { Dry Season TN } \\
\text { Average } \\
\text { Concentration } \pm \\
\text { Standard } \\
\text { Deviation }(\mathrm{mg} / \mathrm{L})\end{array}$ & $\begin{array}{c}\text { Dry Season } \\
\text { TN Average } \\
\text { \% Residual } \pm \\
\text { Standard } \\
\text { Deviation }\end{array}$ \\
\hline Inlet & 1 & 2.8 & 41.6 & $18.3 \pm 12.5$ & - \\
\hline \multirow[t]{3}{*}{$\mathrm{SSF}$} & $1-2$ & 5.5 & 19.7 & $12.0 \pm 4.9$ & $121 \% \pm 133 \%$ \\
\hline & $1-3$ & 3.4 & 19.9 & $12.6 \pm 5.2$ & $111 \% \pm 112 \%$ \\
\hline & $1-4$ & 2.8 & 17.9 & $9.7 \pm 5.0$ & $96 \% \pm 100 \%$ \\
\hline \multirow[t]{3}{*}{$\mathrm{SF}$} & $2-5$ & 2.8 & 7.7 & $5.3 \pm 1.4$ & $50 \% \pm 24 \%$ \\
\hline & $3-6$ & 2.8 & 14.3 & $7.9 \pm 4.0$ & $69 \% \pm 40 \%$ \\
\hline & $4-7$ & 2.8 & 11.3 & $5.9 \pm 3.0$ & $79 \% \pm 80 \%$ \\
\hline \multirow{3}{*}{$\begin{array}{l}\text { Overall CW } \\
(\mathrm{SSF}+\mathrm{SF})\end{array}$} & $1-2-5$ & same as $2-5$ & same as $2-5$ & same as $2-5$ & $56 \% \pm 64 \%$ \\
\hline & $1-3-6$ & same as 3-6 & same as 3-6 & same as 3-6 & $63 \% \pm 56 \%$ \\
\hline & $1-4-7$ & same as 4-7 & same as 4-7 & same as 4-7 & $46 \% \pm 34 \%$ \\
\hline
\end{tabular}




\subsubsection{Phosphorus}

Phosphorus concentrations were not consistently reduced by the CW System, as shown in

Figure 3-5. No trend was shown in the SSF CW results, with much more constant results within the SF CW. These closer results in the SF CW can be attributed to the increased oxygen content in the superficial layer of the SF CW cells, which would enable phosphorus uptake by aerobic microorganisms. Seasonal results are summarized below.

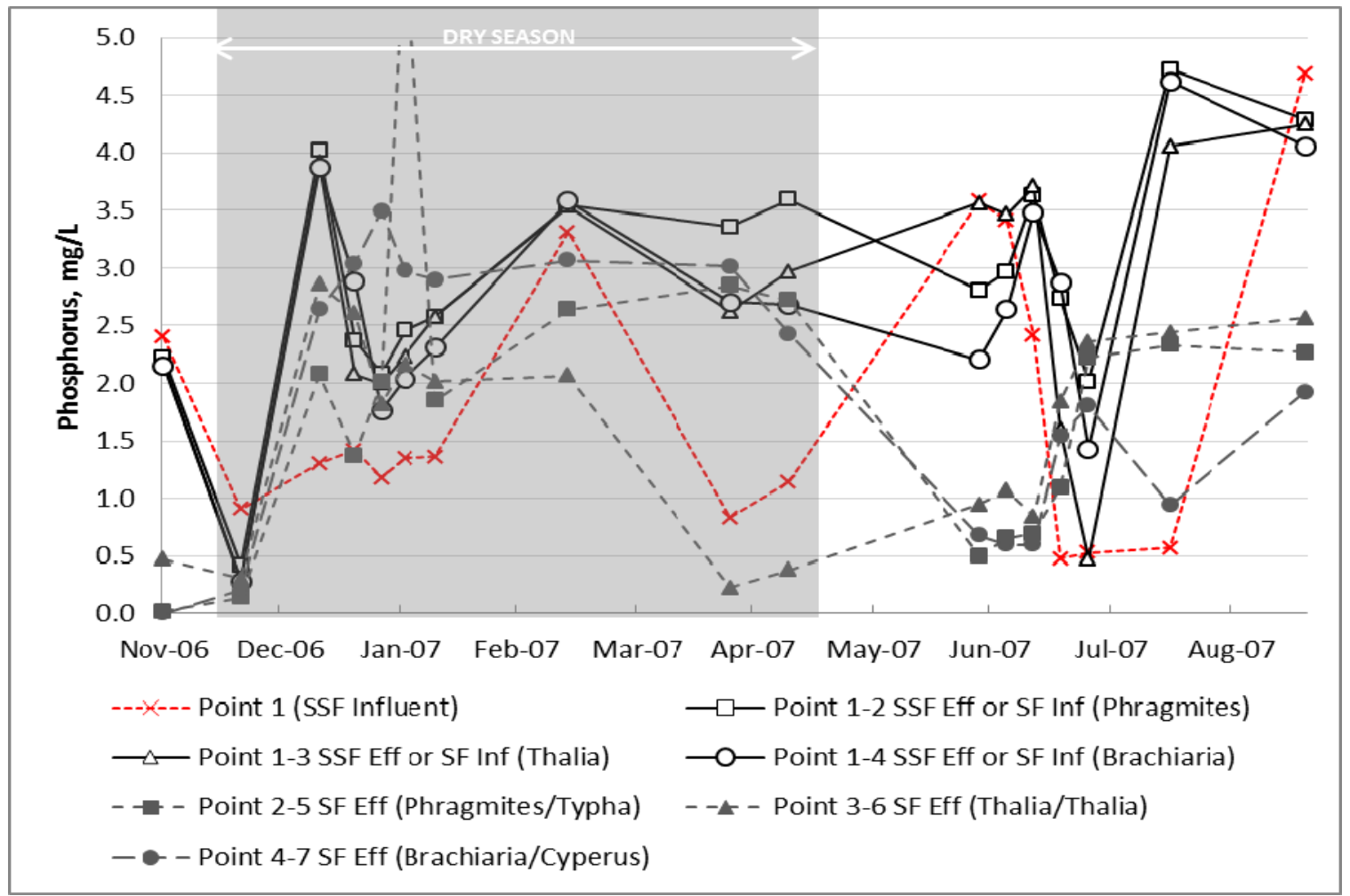

Figure 3-7: Phosphorus Influent vs. Effluent Concentrations

Table 3-13 summarizes the Phosphorus results during the wet season (May to November). The SSF CW did not successfully remove Phosphorus, with its best performance at the Point 1-3 (Inlet/Thalia) still having higher concentration than the inlet concentration (214\% $\pm 207 \%$ residual). Point 2-5 (Phragmites/Typha) presented better results for wet season Phosphorus removal, with an average residual of $43 \% \pm 34 \%$. 
However, when looking at the complete CW System, none of results were satisfactory for Phosphorus removal with its best performance at the Point 1-4-7

(Inlet/Brachiaria/Cyperus) still having higher concentration than the inlet concentration $(127 \% \pm 137 \%$ residual). The achieved results were not consistent with other studies for phosphorus removal, typically phosphorus residual vary from $70 \%$ to $80 \%$ (Brix \& Arias, 2005).

Table 3-13: Phosphorus Wet Season Summary

\begin{tabular}{|c|c|c|c|c|c|}
\hline $\begin{array}{c}\text { Location } \\
\text { Description }\end{array}$ & $\begin{array}{c}\text { Monitoring } \\
\text { Point } \\
\text { Location }\end{array}$ & $\begin{array}{c}\text { Wet Season } \\
\text { Phosphorus } \\
\text { Minimum } \\
\text { Concentration } \\
(\mathrm{mg} / \mathrm{L})\end{array}$ & $\begin{array}{c}\text { Wet Season } \\
\text { Phosphorus } \\
\text { Maximum } \\
\text { Concentration } \\
(\mathrm{mg} / \mathrm{L})\end{array}$ & $\begin{array}{c}\text { Wet Season } \\
\text { Phosphorus } \\
\text { Average } \\
\text { Concentration } \pm \\
\text { Standard } \\
\text { Deviation }(\mathrm{mg} / \mathrm{L})\end{array}$ & $\begin{array}{c}\text { Wet Season } \\
\text { Phosphorus } \\
\text { Average \% } \\
\text { Residual } \pm \\
\text { Standard } \\
\text { Deviation }\end{array}$ \\
\hline Inlet & 1 & 0.5 & 4.7 & $2.1 \pm 1.6$ & - \\
\hline \multirow[t]{3}{*}{ SSF } & $1-2$ & 2.0 & 4.7 & $3.2 \pm 0.9$ & $288 \% \pm 265 \%$ \\
\hline & $1-3$ & 0.5 & 4.3 & $2.9 \pm 1.3$ & $214 \% \pm 207 \%$ \\
\hline & $1-4$ & 1.4 & 4.6 & $2.9 \pm 1.0$ & $264 \% \pm 266 \%$ \\
\hline \multirow[t]{3}{*}{$\mathrm{SF}$} & $2-5$ & 0.0 & 2.7 & $1.4 \pm 1.0$ & $43 \% \pm 34 \%$ \\
\hline & $3-6$ & 0.4 & 2.6 & $1.4 \pm 0.9$ & $95 \% \pm 156 \%$ \\
\hline & $4-7$ & 0.0 & 2.4 & $1.2 \pm 0.8$ & $45 \% \pm 40 \%$ \\
\hline \multirow{3}{*}{$\begin{array}{l}\text { Overall CW } \\
(\mathrm{SSF}+\mathrm{SF})\end{array}$} & $1-2-5$ & same as $2-5$ & same as $2-5$ & same as $2-5$ & $156 \% \pm 172 \%$ \\
\hline & $1-3-6$ & same as 3-6 & same as 3-6 & same as 3-6 & $162 \% \pm 194 \%$ \\
\hline & $1-4-7$ & same as 4-7 & same as $4-7$ & same as $4-7$ & $127 \% \pm 137 \%$ \\
\hline
\end{tabular}

Table 3-14 summarizes the Phosphorus results during the dry season (December to April). The SSF CW did not successfully remove Phosphorus, with its best performance at the Point 1-3 (Inlet/Thalia) still having higher concentration than the inlet concentration $(178 \% \pm 94 \%$ residual). Point 3-6 (Thalia/Thalia) presented better results for dry season Phosphorus removal, with an average residual of $79 \% \pm 35 \%$. However, 
when looking at the complete CW System, none of results were satisfactory for Phosphorus removal with its best performance at the Point 1-3-6 (Inlet/Thalia/Thalia) still having higher concentration than the inlet concentration $(124 \% \pm 73 \%$ residual).

Table 3-14: Phosphorus Dry Season Summary

\begin{tabular}{|c|c|c|c|c|c|}
\hline $\begin{array}{c}\text { Location } \\
\text { Description }\end{array}$ & $\begin{array}{c}\text { Monitoring } \\
\text { Point } \\
\text { Location }\end{array}$ & $\begin{array}{c}\text { Dry Season } \\
\text { Phosphorus } \\
\text { Minimum } \\
\text { Concentration } \\
(\mathrm{mg} / \mathrm{L})\end{array}$ & $\begin{array}{c}\text { Dry Season } \\
\text { Phosphorus } \\
\text { Maximum } \\
\text { Concentration } \\
(\mathrm{mg} / \mathrm{L})\end{array}$ & $\begin{array}{c}\text { Dry Season } \\
\text { Phosphorus } \\
\text { Average } \\
\text { Concentration } \pm \\
\text { Standard } \\
\text { Deviation }(\mathrm{mg} / \mathrm{L})\end{array}$ & $\begin{array}{c}\text { Dry Season } \\
\text { Phosphorus } \\
\text { Average \% } \\
\text { Residual } \pm \\
\text { Standard } \\
\text { Deviation }\end{array}$ \\
\hline Inlet & 1 & 0.8 & 3.3 & $1.5 \pm 0.8$ & - \\
\hline \multirow[t]{3}{*}{$\mathrm{SSF}$} & $1-2$ & 0.4 & 4.0 & $2.6 \pm 1.1$ & $198 \% \pm 112 \%$ \\
\hline & $1-3$ & 0.3 & 3.9 & $2.4 \pm 1.1$ & $178 \% \pm 94 \%$ \\
\hline & $1-4$ & 0.3 & 3.9 & $2.4 \pm 1.1$ & $179 \% \pm 96 \%$ \\
\hline \multirow[t]{3}{*}{ SF } & $2-5$ & 0.1 & 6.0 & $2.4 \pm 1.7$ & $89 \% \pm 65 \%$ \\
\hline & 3-6 & 0.2 & 2.9 & $1.8 \pm 1.0$ & $79 \% \pm 35 \%$ \\
\hline & $4-7$ & 0.2 & 3.5 & $2.7 \pm 1.0$ & $114 \% \pm 43 \%$ \\
\hline \multirow{3}{*}{$\begin{array}{l}\text { Overall CW } \\
(\mathrm{SSF}+\mathrm{SF})\end{array}$} & $1-2-5$ & same as $2-5$ & same as $2-5$ & same as $2-5$ & $180 \% \pm 142 \%$ \\
\hline & $1-3-6$ & same as 3-6 & same as 3-6 & same as 3-6 & $124 \% \pm 73 \%$ \\
\hline & $1-4-7$ & same as 4-7 & same as 4-7 & same as 4-7 & $203 \% \pm 107 \%$ \\
\hline
\end{tabular}

\subsubsection{Plant growth and health}

Plant appearance and health were evaluated over the course of the monitoring period.

Care was taken such that all plants used for assessment purposes appeared healthy, with no indication of any sort of pathology. All plants had developed strong root systems within the wetland soil and SSF gravel and were uniform in growth.

Biomass samples were analyzed for nitrogen and phosphorus mineral content. Fastgrowing Brachiaria proved especially proficient, with $\mathrm{N}$ and $\mathrm{P}$ content of $1.5-3.14 \%$ and $0.17-0.25 \%$ per dry plants' biomass, respectively. This plant was harvested three times 
during the observational period for use as a fodder for horses and cows. Following transplantation, Thalia growth and nutrient content closely matched that of Brachiaria (N: 1.7-2.4\%, P: 0.2-0.37\%). Thalia high P content coincides with year-round enhanced Total Phosphorus mass removal efficiency through Points 1-3-6 (Inlet/Thalia/Thalia). However, Thalia requires frequent trimming and after an initial prolific stage it tends to undergo senescence, reducing its capacity to effect contaminant removal. While Cyperus had a robust, healthy appearance with respective $\mathrm{N}$ and $\mathrm{P}$ content of $1.1-1.15 \%$ and $0.14-$ $0.17 \%$, it proved particularly difficult to maintain. It was more sensitive than either Typha or Thalia to changes in water availability, not tolerating water levels greater than 15-20 $\mathrm{cm}$ during the growth period. When the water was maintained at $30 \mathrm{~cm}$, growth was inhibited and the vegetation faltered, becoming so overtaken by weeds that it became necessary to replant the entire cell. Phragmites boasts a well-developed root system and grows vigorously under wetland conditions with relatively little maintenance. It did not require many trimmings, facilitated respective nitrogen and phosphorus content of 1.3$2.1 \%$ and $0.13-0.24 \%$ and was unaffected by any plagues and/or diseases. Typha, a plant endemic to most natural wetlands, is characterized by well-developed roots, rapid growth and significant nutrient content (N: 0.8-2.2\%, P: 0.12-0.3\%) (Katsenovich, et al., 2009). The dry plant composition for nitrogen and phosphorus are summarized in Table 3-15. 
Table 3-15: Nitrogen and Phosphorus Dry Plant Composition

\begin{tabular}{|l|l|l|}
\hline Plant Species & $\begin{array}{l}\text { Nitrogen Content in } \\
\text { dry plant } \%\end{array}$ & $\begin{array}{l}\text { Phosphorus Content } \\
\text { in dry plant \% }\end{array}$ \\
\hline Brachiaria & $1.5-3.1 \%$ & $0.17-0.25 \%$ \\
\hline Thalia* & $1.7-2.4 \%$ & $0.20-0.37 \%$ \\
\hline Cyperus & $1.1-1.2 \%$ & $0.14-0.17 \%$ \\
\hline Phragmites & $1.3-2.1 \%$ & $0.13-0.24 \%$ \\
\hline Typha & $0.8-2.2 \%$ & $0.12-0.30 \%$ \\
\hline * after transplantation & & \\
\hline
\end{tabular}

For comparison purposes, some studies estimated that only 5 to $10 \%$ of the nitrogen load is absorbed by the plants (WEF, 2000), with nitrogen plant content as $4 \%$ of nitrogen input depending on the plant species (Kantawanichkul, et al., 2003). Phragmites had a $7 \%$ nitrogen content by plants in another study (Tuncsiper, 2009). All the previous studies nitrogen content results were significantly higher than the ones obtained in study.

The nitrogen and phosphorus plant biomass per season are presented in Table 3-16. It is noted that both nitrogen and phosphorus were higher during the dry season for the SSF plants. The SSF nitrogen biomass ranged from $19-46 \mathrm{~g} / \mathrm{m}^{2}$ and $2.6-14.8 \mathrm{~g} / \mathrm{m}^{2}$ during the dry and wet seasons, respectively. The SSF phosphorus biomass ranged from $1.8-$ $5.0 \mathrm{~g} / \mathrm{m}^{2}$ and $0.4-2.0 \mathrm{~g} / \mathrm{m}^{2}$ during the dry and wet seasons, respectively. It is noted that both nitrogen and phosphorus were higher during the wet season for the SF plants. The SF nitrogen biomass ranged from $3.4-8.2 \mathrm{~g} / \mathrm{m}^{2}$ and $12.5-15.4 \mathrm{~g} / \mathrm{m}^{2}$ during the dry and wet seasons, respectively. The SF phosphorus biomass ranged from $0.4-1.2 \mathrm{~g} / \mathrm{m}^{2}$ and 
$1.6-2.5 \mathrm{~g} / \mathrm{m}^{2}$ during the dry and wet seasons, respectively. The accumulated nutrients (nitrogen and phosphorus) can be released back to the water during plant senescence, which affects the effluent concentrations in the CW system.

Table 3-16: Plant Biomass Characteristics

\begin{tabular}{|c|c|c|c|c|c|}
\hline \multirow[t]{2}{*}{$\mathrm{CW}$} & \multirow[t]{2}{*}{ Plant Species } & \multicolumn{2}{|l|}{ Nitrogen, $\mathrm{g} / \mathrm{m}^{2}$} & \multicolumn{2}{|c|}{ Phosphorus, $\mathrm{g} / \mathrm{m}^{2}$} \\
\hline & & Dry Season* & Wet Season** & Dry Season* & Wet Season** \\
\hline \multirow[t]{3}{*}{ SSF } & Phragmites & 19.03 & 14.79 & 1.76 & 1.95 \\
\hline & SSF Thalia & 45.84 & 7.81 & 4.96 & 1.02 \\
\hline & Brachiaria & 40.21 & 2.61 & 4.14 & 0.44 \\
\hline \multirow[t]{3}{*}{ SF } & Typha & 8.15 & 13.94 & 1.18 & 1.59 \\
\hline & SF Thalia & 4.21 & 15.44 & 0.67 & 2.52 \\
\hline & Cyperus & 3.42 & 12.51 & 0.40 & 1.87 \\
\hline
\end{tabular}

Other studies reported nitrogen biomass between 0.6 and $72 \mathrm{~g} / \mathrm{m}^{2}$, with an average of $20.7 \mathrm{~g} / \mathrm{m}^{2}$ (Johnston, 1991), and also ranging between 5.3 and $58.7 \mathrm{~g} / \mathrm{m}^{2}$ for various types of constructed wetlands and plants (Vymazal \& Kropfelova, 2008). Phosphorus biomass has been reported ranging from 0.2 to $10.5 \mathrm{~g} / \mathrm{m}^{2}$ for various types of wetlands as well (Vymazal, 2004). 


\subsection{Comparative Assessment of Models Used During Design}

The models used to determine the SSF and SF CW areas during design, presented in Chapter 2.1.3, were re-evaluated using the actual constructed SSF and SF areas, water balance and quality data obtained during the monitoring period. The actual data results were then compared with the design results to evaluate the existing constructed wetland models effectiveness for $\mathrm{BOD}_{5}$, and TSS removals. Total Nitrogen models were evaluated for SF CW only. Phosphorus, Fecal coliform, COD, and Oil \& Grease were not used to size the SSF and the SF CW cells.

\subsubsection{SSF CW}

As described in Chapter 2.1.3, each SSF cell has an area of $151.20 \mathrm{~m}^{2}$, water depth of 0.8 $\mathrm{m}$, and gravel porosity of $0.4 \mathrm{~m}^{3} / \mathrm{m}^{3}$. Chapter 2.1.3.1 (Table 2-2, Table 2-3 and Table 2-4) presents the target parameters and treatment objectives used on the design of this study CW system.

\subsubsection{1 $\underline{\mathrm{BOD}_{5}}$}

Kadlec \& Knight design calculation used a first-order areal rate constant (k) of 180 m/yr, but the actual average $\mathrm{k}$ for all the three cells was $20 \mathrm{~m} / \mathrm{yr} \pm 18 \mathrm{~m} / \mathrm{yr}$, considered significantly lower than the $\mathrm{k}$ used in the design. The $\mathrm{BOD}_{5}$ background concentration used in the design calculation was $7.4 \mathrm{mg} / \mathrm{L}$, and the actual average background concentration was $7.0 \mathrm{mg} / \mathrm{L} \pm 1.4 \mathrm{mg} / \mathrm{L}$. As shown in Figure 3-8, the design BOD target outlet (effluent) concentration based on the Kadlec \& Knight design values and actual 
SSF dimensions, is much lower than the $\mathrm{BOD}_{5}$ actual outlet (effluent) concentrations. Had a much lower $\mathrm{k}$ value been used for the area calculation, a much larger SSF CW would be required.

Campbell \& Ogden and Reed et.al design calculation used a temperature of 20.4 degree Celsius $\left({ }^{\circ} \mathrm{C}\right)$, but the actual average inlet (influent) temperature was $25.8^{\circ} \mathrm{C} \pm 1.4^{\circ} \mathrm{C}$, considered significantly higher than the influent temperature used in the design. As shown in Figure 3-8, the design $\mathrm{BOD}_{5}$ target outlet (effluent) concentration based on the Campbell \& Ogden and Reed et. al design values and actual SSF dimensions, is much lower than the $\mathrm{BOD}_{5}$ actual outlet (effluent) concentrations. Had a higher influent temperature been used for the area calculation, a smaller SSF CW would be required ( 25 to $30 \%$ smaller).

The EPA design calculation used an areal loading rate (ALR) of $1.6 \mathrm{~g} / \mathrm{m}^{2}-\mathrm{d}$ to attain 20 $\mathrm{mg} / \mathrm{L}$ effluent concentration (which required a much larger SSF CW area than the actual constructed area). The actual ALR ranged from 2.0 to $11.6 \mathrm{~g} / \mathrm{m}^{2}-\mathrm{d}$, with an average of $6.0 \mathrm{~g} / \mathrm{m}^{2}-\mathrm{d} \pm 3.0 \mathrm{~g} / \mathrm{m}^{2}-\mathrm{d}$. According to the EPA Methodology, an areal loading rate of 6.0 $\mathrm{g} / \mathrm{m}^{2}$-d would attain $30 \mathrm{mg} / \mathrm{L}$ effluent, which was higher effluent concentration than the treated effluent objective of $20 \mathrm{mg} / \mathrm{L}$. As shown in Figure 3-9, even though the treated effluent objective was $20 \mathrm{mg} / \mathrm{L}$, some of the sampling events achieved the result expected from the EPA Methodology for $30 \mathrm{mg} / \mathrm{L}$ with lower areal loading rates.

Based on the current model results presented in this Chapter, and had the treated effluent objective been $30 \mathrm{mg} / \mathrm{L}$ (not $20 \mathrm{mg} / \mathrm{L}$ as per design), the EPA Methodology seems to have achieved more realistic results than the other methodologies. 
It is generally assumed that constructed wetland systems are more efficient in tropical climates than in temperate climates because higher temperatures encourage plant growth and microbial activities, but it is not always the case since higher temperatures are also conducive for secondary contamination. Figure 3-8 and Figure 3-9 clearly show that longer retention times at low BOD areal loading rates during the dry season doesn't improve the water quality; it is actually the opposite and at high tropical temperatures, the $\mathrm{BOD}_{5}$ parameter actually gets worse. It might be attributed to the secondary contamination coming from decaying falling leaves and accumulation of metabolites produced by bacteria and soil biota. 


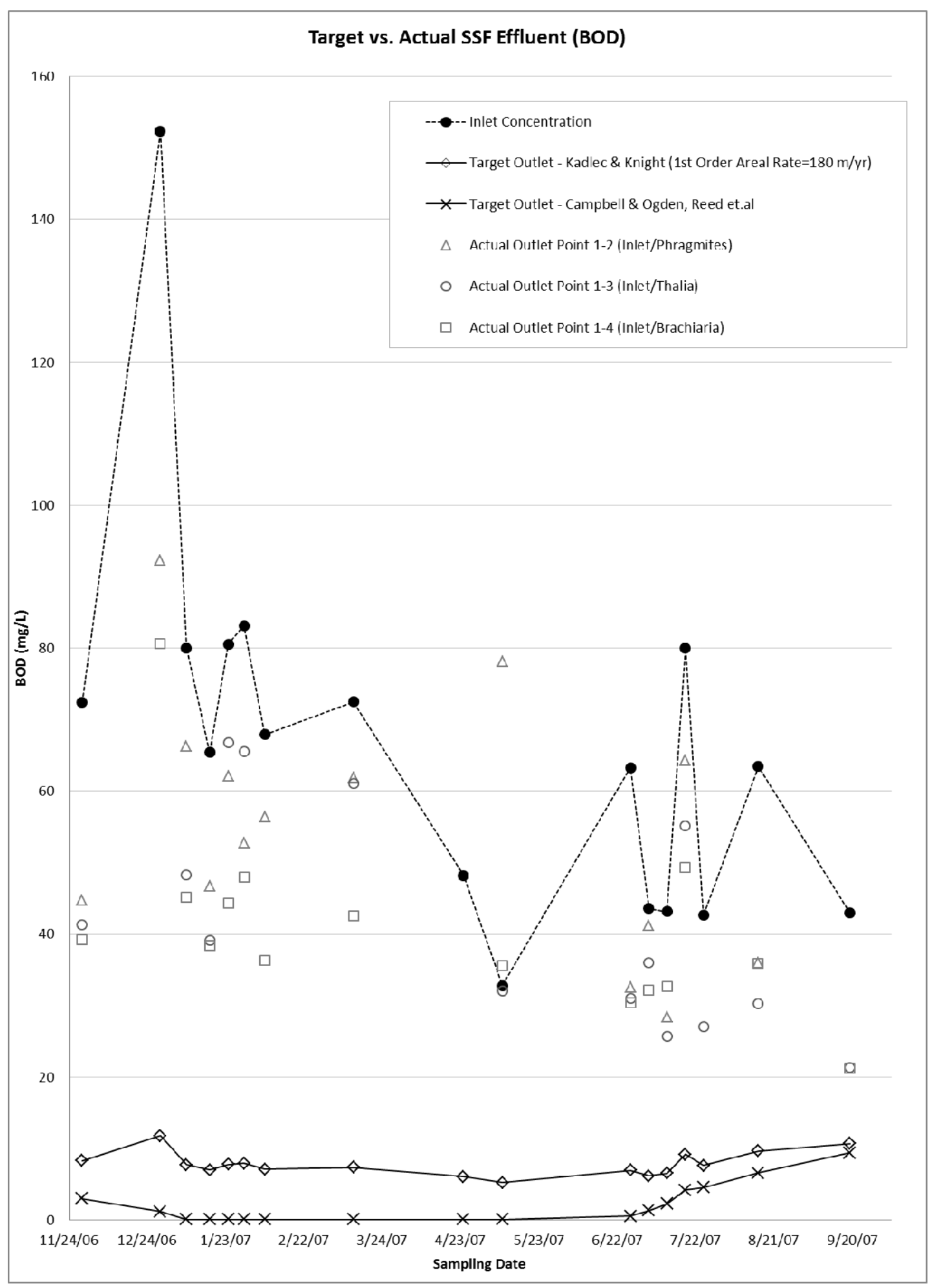

Figure 3-8: BOD $_{5}$ Design Target vs. Actual Effluent Concentrations - Kadlec \& Knight, Campbell \& Ogden, and Reed et.al (SSF CW) 


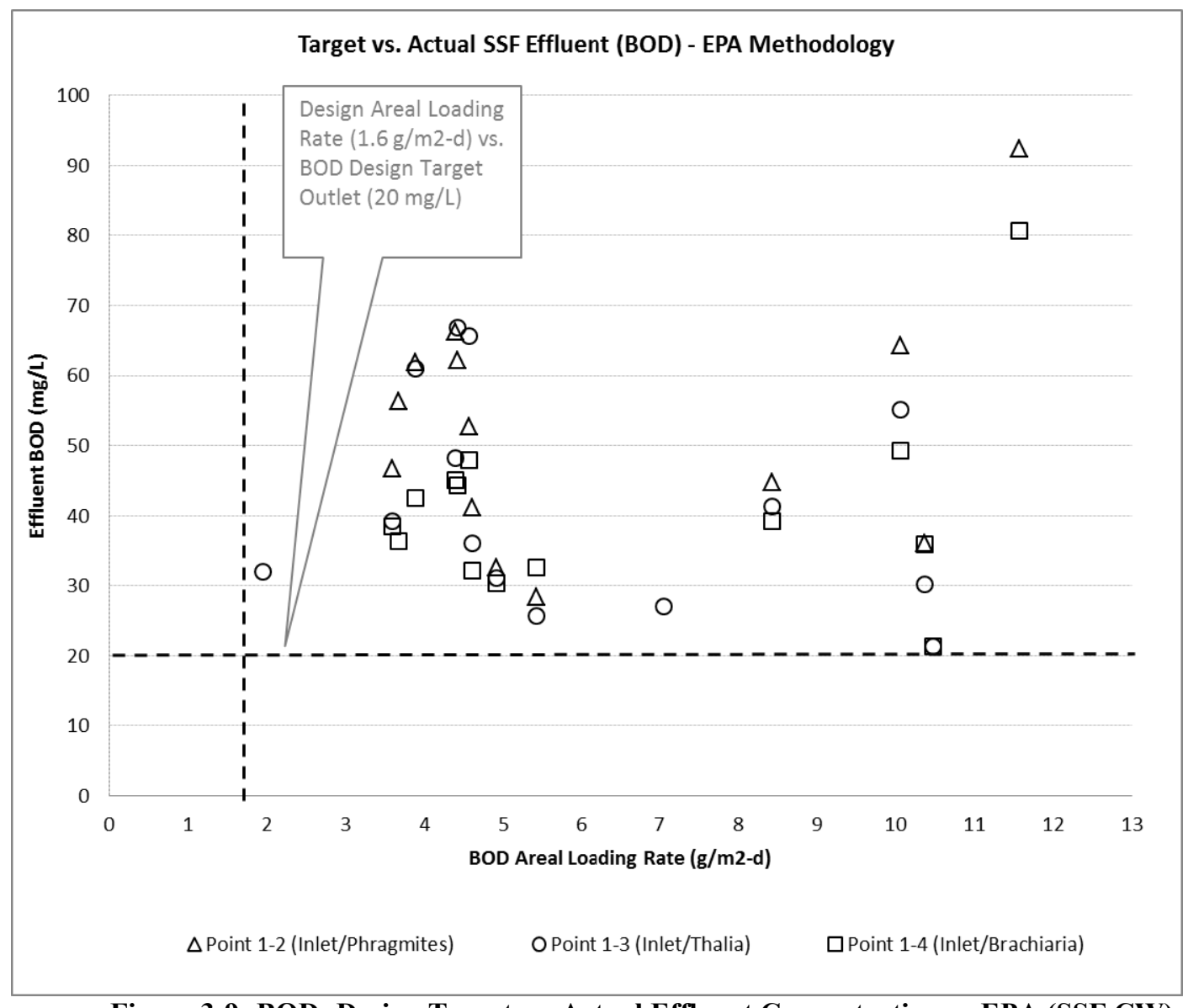

Figure 3-9: BOD $_{5}$ Design Target vs. Actual Effluent Concentrations - EPA (SSF CW)

\subsubsection{2 $\underline{\text { TSS }}$}

Kadlec \& Knight design calculation used a first-order areal rate constant (k) of 3,000 $\mathrm{m} / \mathrm{yr}$, but the actual average $\mathrm{k}$ for all the three cells was $92 \mathrm{~m} / \mathrm{yr} \pm 46 \mathrm{~m} / \mathrm{yr}$, considered significantly lower than the $\mathrm{k}$ used in the design. The TSS background concentration used in the design calculation was $16.5 \mathrm{mg} / \mathrm{L}$, and the actual average background concentration was $18 \mathrm{mg} / \mathrm{L} \pm 9 \mathrm{mg} / \mathrm{L}$. As shown in Figure 3-10, the design TSS target outlet (effluent) concentration based on the Kadlec \& Knight design values and actual SSF dimensions, is different than the TSS actual outlet (effluent) concentrations, but 
within an acceptable range. Had a much lower $\mathrm{k}$ value been used for the area calculation, a much larger SF CW would be required.

Campbell \& Ogden design calculation used a hydraulic loading rate (HLR) of $35.3 \mathrm{~cm} / \mathrm{d}$, but the actual HLR was $8.7 \mathrm{~cm} / \mathrm{d} \pm 4.1 \mathrm{~cm} / \mathrm{d}$, considered significantly lower than the HLR used in the design. As shown in Figure 3-10 and Figure 3-11, the design TSS target outlet (effluent) concentration based on the Campbell \& Ogden design values and actual SSF dimensions, is different than the TSS actual outlet (effluent) concentrations, but within an acceptable range. Had a lower HLR been used for the area calculation, a much larger SSF CW would be required (four times larger).

The EPA Methodology calculation recommends an areal loading rate (ALR) of $20 \mathrm{~g} / \mathrm{m}^{2}-\mathrm{d}$ to attain $30 \mathrm{mg} / \mathrm{L}$ effluent concentration. The actual ALR ranged from 3.1 to $35.7 \mathrm{~g} / \mathrm{m}^{2}$ $\mathrm{d}$, with an average of $11.4 \mathrm{~g} / \mathrm{m}^{2}-\mathrm{d} \pm 7.8 \mathrm{~g} / \mathrm{m}^{2}-\mathrm{d}$, considered lower than the areal loading rate used in the design. However, as shown in Figure 3-12, some of the sampling events achieved the result expected from the EPA Methodology (close to $30 \mathrm{mg} / \mathrm{L}$ ) but with lower areal loading rates.

Based on the current model results presented in this Chapter, all three (3) methodologies seem to have achieved results somewhat closer to the actual results. 


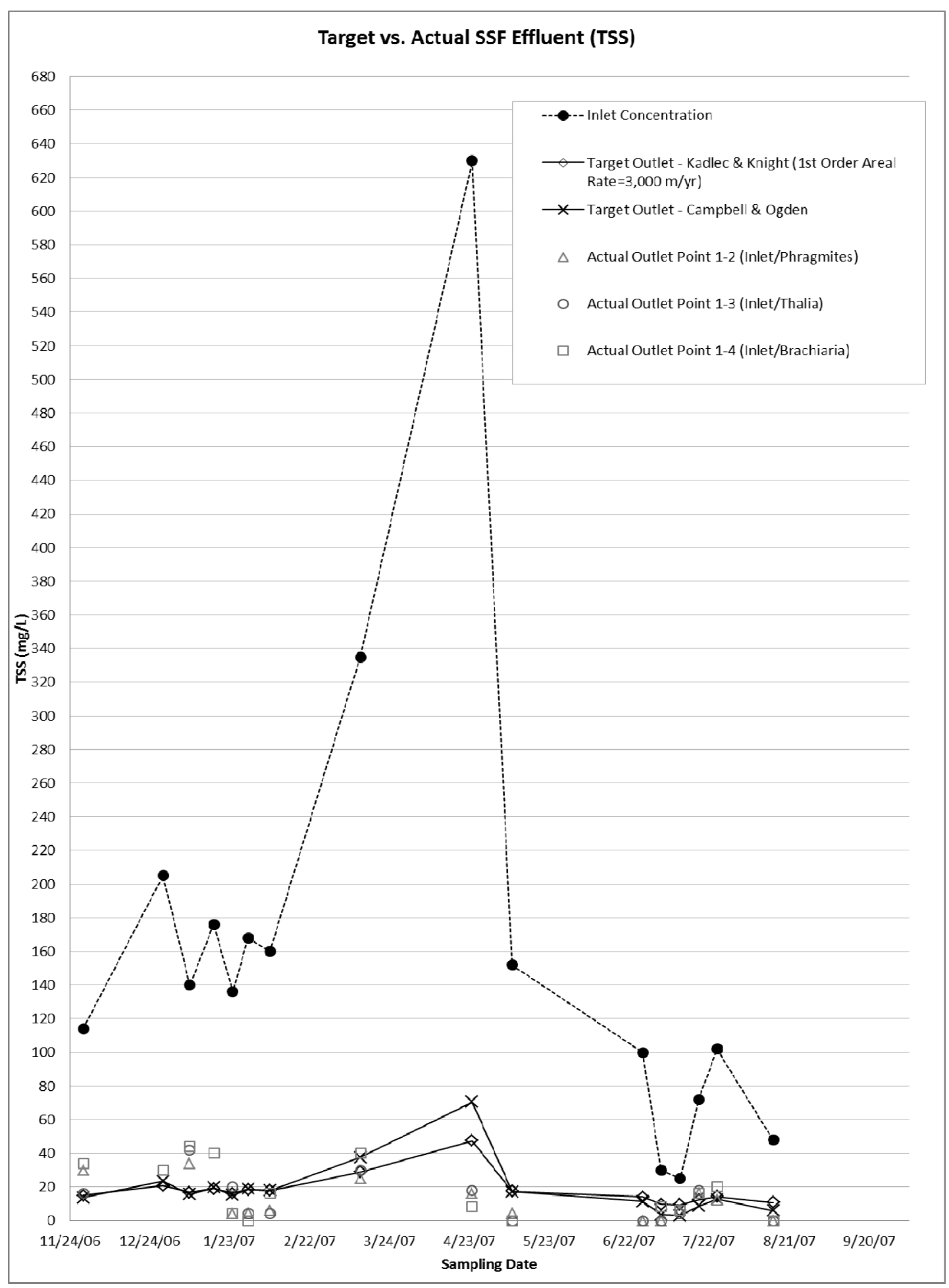

Figure 3-10: TSS Design Target vs. Actual Effluent Concentrations - Kadlec \& Knight, Campbell \& Ogden (SSF CW) 


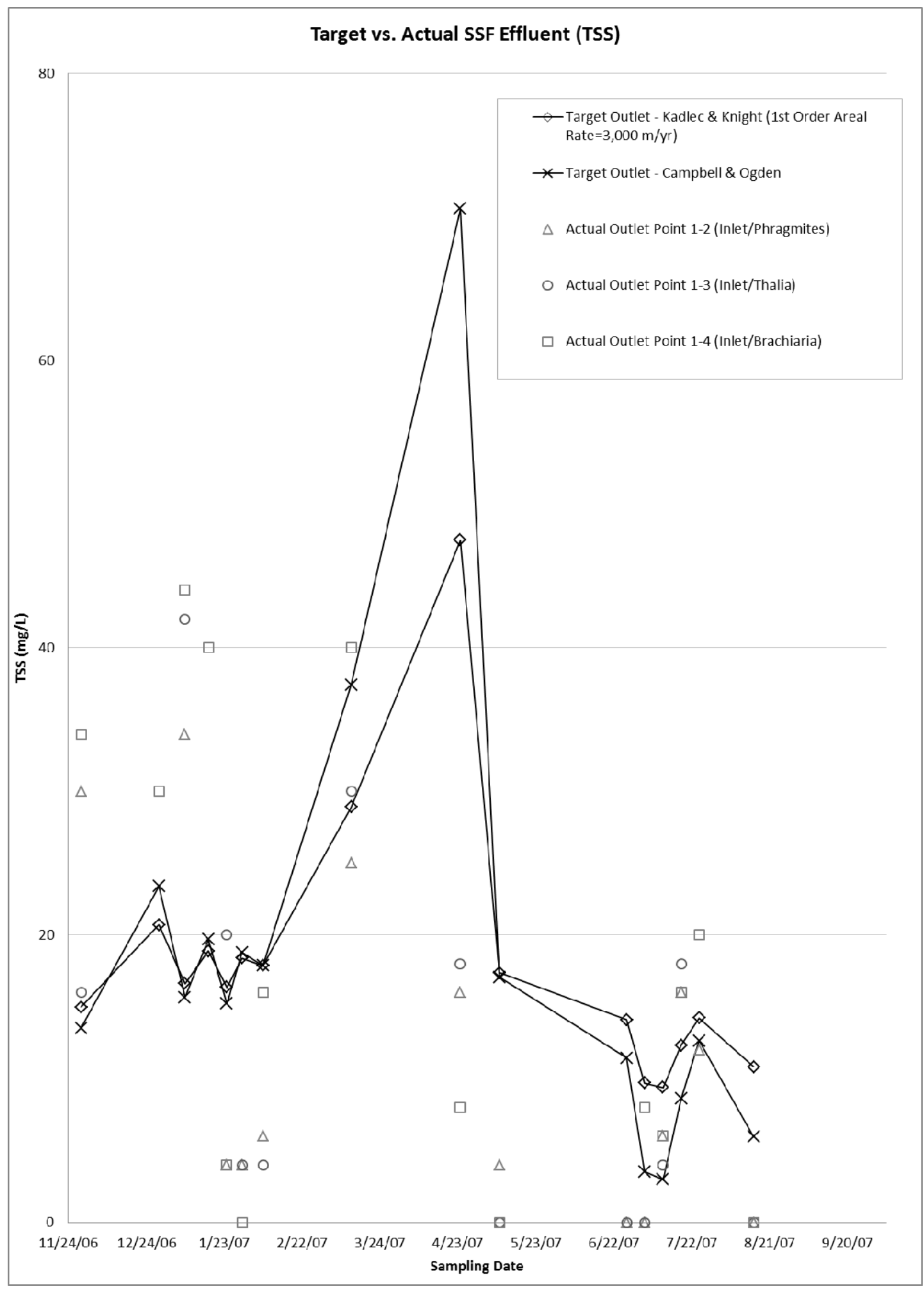

Figure 3-11: TSS Design Target vs. Actual Effluent Concentrations - Kadlec \& Knight, Campbell \& Ogden (Closer View) (SSF CW) 


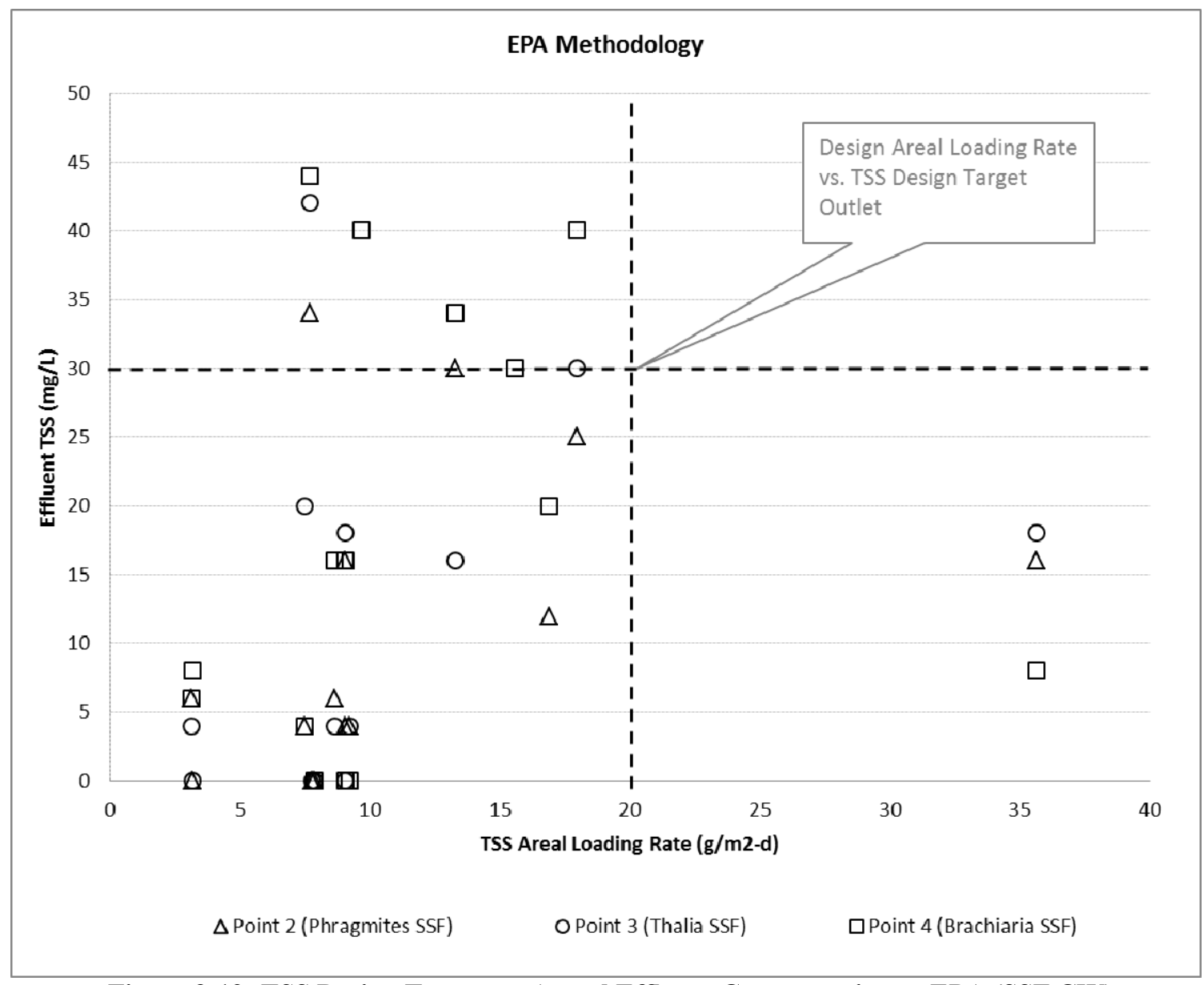

Figure 3-12: TSS Design Target vs. Actual Effluent Concentrations - EPA (SSF CW)

\subsubsection{SF CW}

As described in Chapter 2.1.3, each SF cell has an area of approximately $800 \mathrm{~m}^{2}$ (604 $\mathrm{m}^{2}$ SF plus $195 \mathrm{~m}^{2} \mathrm{OW}$ ), water depths of $0.6 \mathrm{~m}$ and $1.2 \mathrm{~m}$, and gravel porosity of 0.65 $\mathrm{m}^{3} / \mathrm{m}^{3}$ and $0.8 \mathrm{~m}^{3} / \mathrm{m}^{3}$, respectively for the SF and OW cells.

\section{$3.2 .2 .1 \quad \underline{B O D}_{\underline{5}}$}

Kadlec \& Knight design calculation used a first-order areal rate constant (k) of $34 \mathrm{~m} / \mathrm{yr}$, but the actual average $\mathrm{k}$ for all the three cells was $7 \mathrm{~m} / \mathrm{yr} \pm 10 \mathrm{~m} / \mathrm{yr}$, considered 
significantly lower than the $\mathrm{k}$ used in the design. The $\mathrm{BOD}_{5}$ background concentration used in the design calculation was $5.1 \mathrm{mg} / \mathrm{L}$, and the actual average background concentration was $6.1 \mathrm{mg} / \mathrm{L} \pm 1.3 \mathrm{mg} / \mathrm{L}$. As shown in Figure 3-13, the design BOD target outlet (effluent) concentration based on the Kadlec \& Knight design values and actual SSF dimensions, is much lower than the $\mathrm{BOD}_{5}$ actual outlet (effluent) concentrations. Had a much lower $\mathrm{k}$ value been used for the area calculation, a much larger SSF CW would be required.

Campbell \& Ogden and Reed et.al design calculation used a temperature of 20.4 degree Celsius $\left({ }^{\circ} \mathrm{C}\right)$, but the actual average inlet (influent) temperature was $25.8^{\circ} \mathrm{C} \pm 1.4^{\circ} \mathrm{C}$, considered significantly higher than the influent temperature used in the design. As shown in Figure 3-13, the design $\mathrm{BOD}_{5}$ target outlet (effluent) concentration based on the Campbell \& Ogden and Reed et. al design values and actual SF dimensions, is much lower than the $\mathrm{BOD}_{5}$ actual outlet (effluent) concentrations.

The EPA design calculation used an areal loading rate (ALR) of $4.5 \mathrm{~g} / \mathrm{m}^{2}-\mathrm{d}$ to attain 20 $\mathrm{mg} / \mathrm{L}$ effluent concentration. The actual ALR ranged from 0.2 to $2.0 \mathrm{~g} / \mathrm{m}^{2}-\mathrm{d}$, with an average of $0.69 \mathrm{~g} / \mathrm{m}^{2}-\mathrm{d} \pm 0.45 \mathrm{~g} / \mathrm{m}^{2}-\mathrm{d}$, considered much lower than the areal loading recommended by EPA to attain $20 \mathrm{mg} / \mathrm{L}$ BOD effluent. However, as shown in Figure $3-14$, most of the sampling events achieved the results below $20 \mathrm{mg} / \mathrm{L}$ with lower areal loading rates than the EPA number.

Based on the current model results presented in this Chapter, the EPA Methodology seems to have achieved better results than the other methodologies. 
As mentioned in the Section 3.2.1.1, constructed wetland systems are expected to be more efficient in tropical climates than in temperate climates because higher temperatures encourage plant growth and microbial activities; however, higher temperatures are conducive for secondary contamination which may worsen the $\mathrm{BOD}_{5}$ effluent concentration due to decaying falling leaves and accumulation of metabolites produced by bacteria and soil biota. 


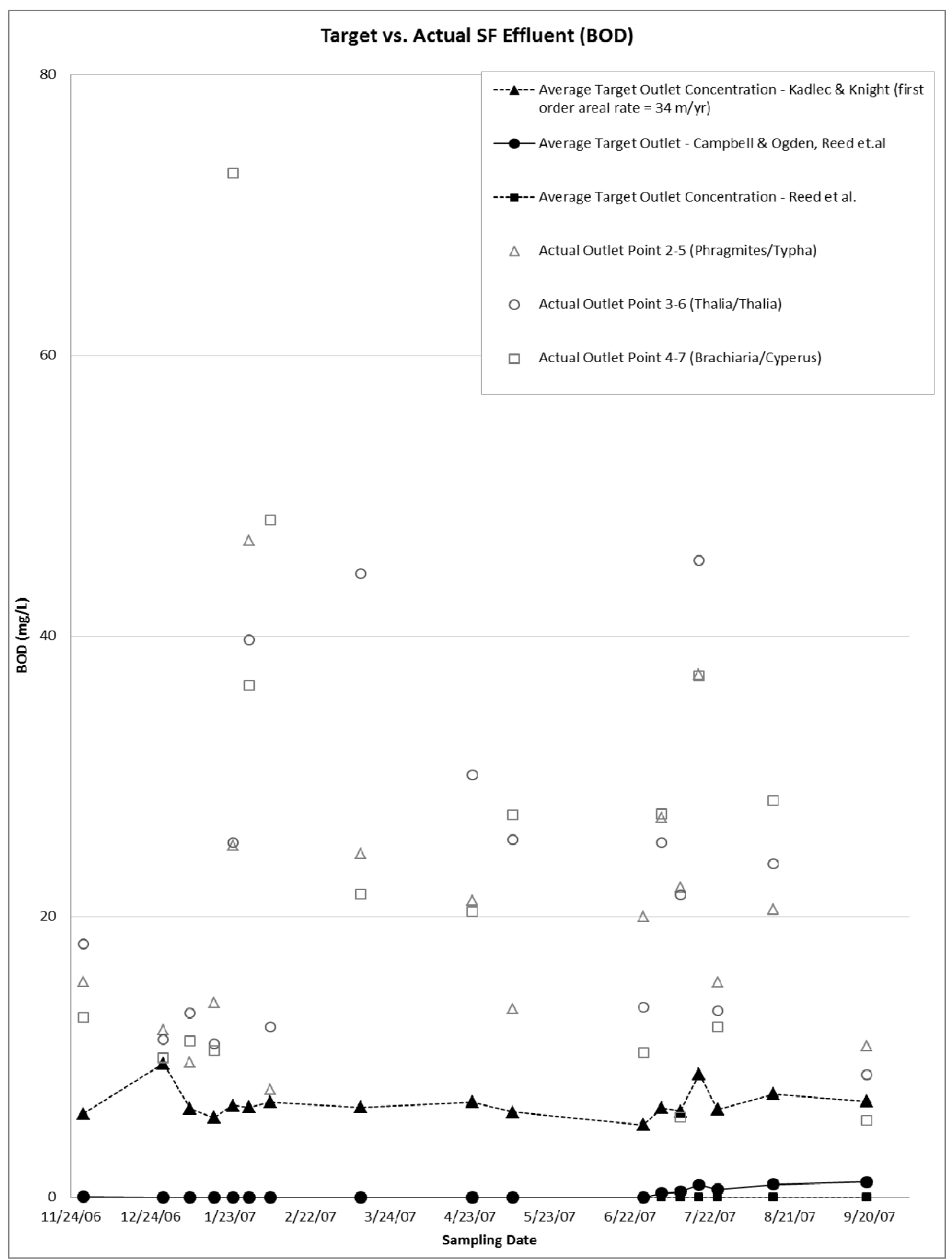

Figure 3-13: BOD 5 Design Target vs. Actual Effluent Concentrations - Kadlec \& Knight, Campbell \& Ogden, and Reed et.al (SF CW) 


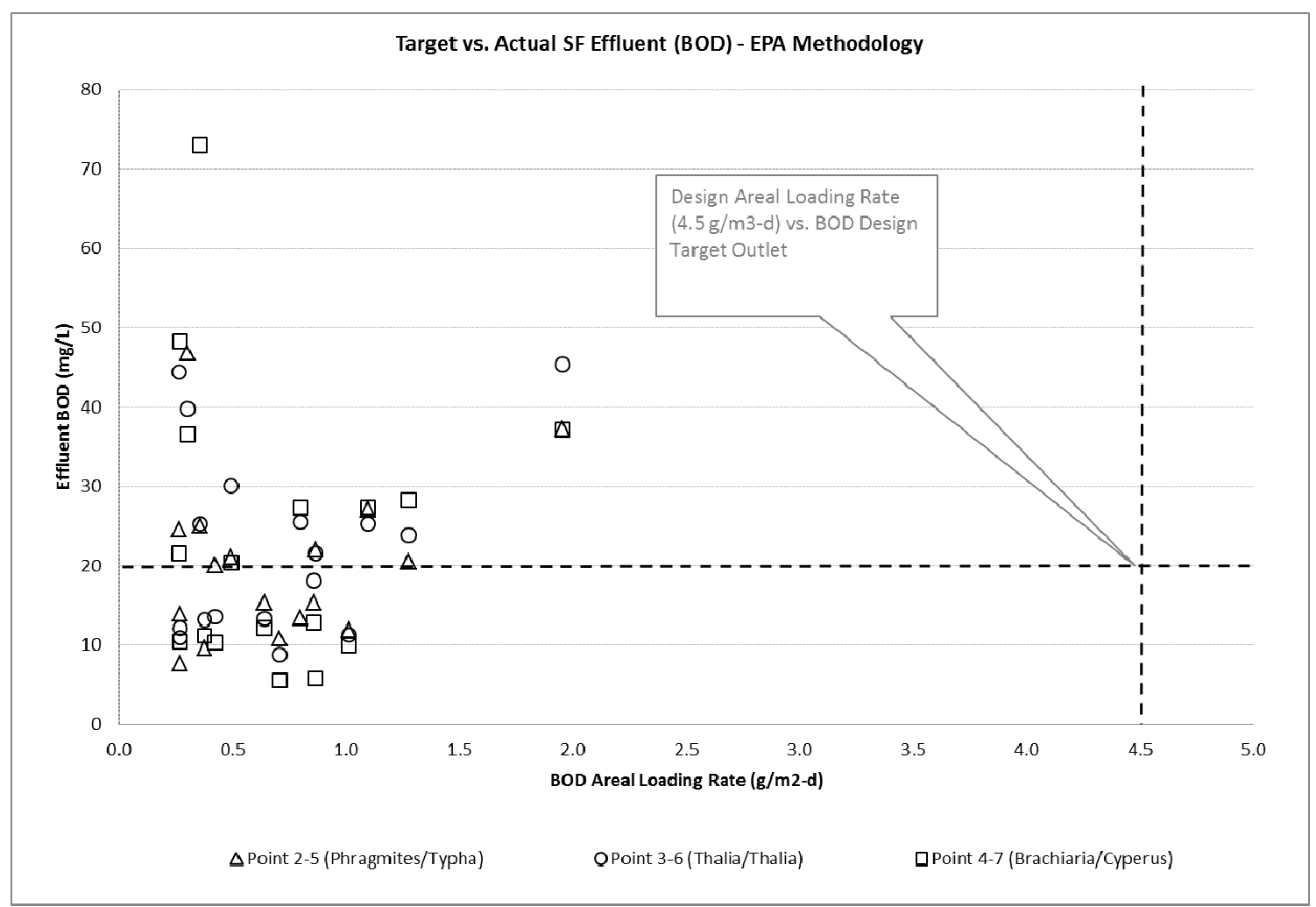

Figure 3-14: BOD $_{5}$ Design Target vs. Actual Effluent Concentrations - EPA (SF CW)

\subsubsection{2 $\underline{\text { TSS }}$}

Kadlec \& Knight design calculation used a first-order areal rate constant (k) of 1,000 $\mathrm{m} / \mathrm{yr}$, but the actual average $\mathrm{k}$ for all the three cells was $4.2 \mathrm{~m} / \mathrm{yr} \pm 10.3 \mathrm{~m} / \mathrm{yr}$, considered significantly lower than the $\mathrm{k}$ used in the design. The TSS background concentration used in the design calculation was $14.7 \mathrm{mg} / \mathrm{L}$, and the actual average background concentration was $4.6 \mathrm{mg} / \mathrm{L} \pm 1.3 \mathrm{mg} / \mathrm{L}$. As shown in Figure 3-15, the design TSS target outlet (effluent) concentration based on the Kadlec \& Knight design values and actual SSF dimensions, is different than the TSS actual outlet (effluent) concentrations, but within an acceptable range. Had a much lower k value been used for the area calculation, a much larger SSF CW would be required. 
Campbell \& Ogden design calculation used a hydraulic loading rate (HLR) of $68.1 \mathrm{~cm} / \mathrm{d}$, but the actual HLR was $1.8 \mathrm{~cm} / \mathrm{d} \pm 1.5 \mathrm{~cm} / \mathrm{d}$, considered significantly lower than the HLR used in the design. As shown in Figure 3-15 and Figure 3-16, the design TSS target outlet (effluent) concentration based on the Campbell \& Ogden design values and actual SSF dimensions, is different than the TSS actual outlet (effluent) concentrations, but within an acceptable range. Had a lower HLR been used for the area calculation, a much larger SSF CW would be required.

The EPA design calculation used an areal loading rate (ALR) of $3 \mathrm{~g} / \mathrm{m}^{2}-\mathrm{d}$ to attain 20 $\mathrm{mg} / \mathrm{L}$ effluent concentration. The actual ALR ranged from 0.0 to $1.6 \mathrm{~g} / \mathrm{m}^{2}-\mathrm{d}$, with an average of $0.24 \mathrm{~g} / \mathrm{m}^{2}-\mathrm{d} \pm 0.32 \mathrm{~g} / \mathrm{m}^{2}-\mathrm{d}$, considered lower than the areal loading rate recommended by EPA to attain $20 \mathrm{mg} / \mathrm{L}$. However, as shown in Figure 3-17, most of the sampling events achieved result better than the expected from the EPA Methodology (close to $20 \mathrm{mg} / \mathrm{L}$ ) but with lower areal loading rates.

Based on the current model results presented in this Chapter, all three (3) methodologies seem to have achieved results somewhat closer to the actual results. 


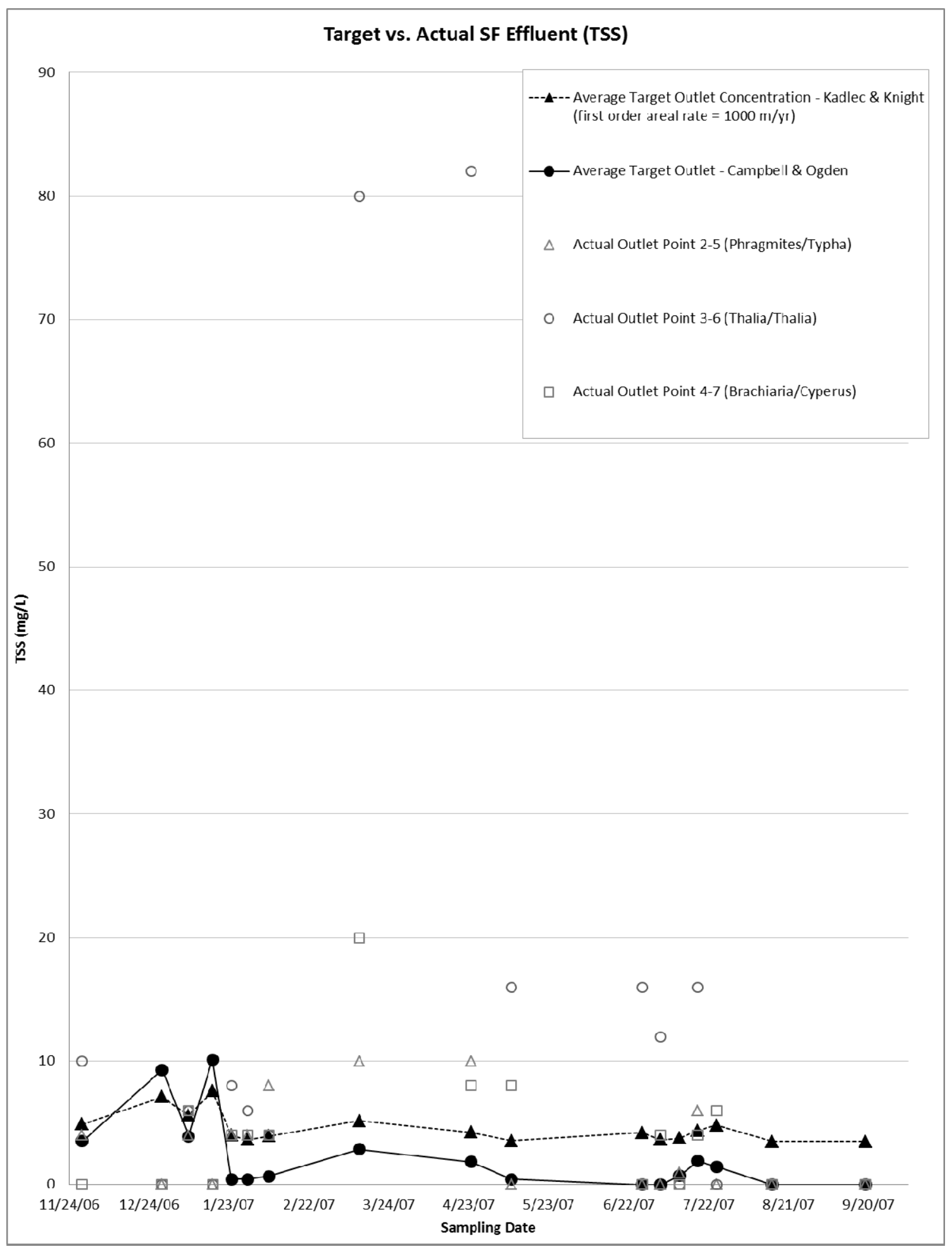

Figure 3-15: TSS Design Target vs. Actual Effluent Concentrations - Kadlec \& Knight, Campbell \& Ogden 


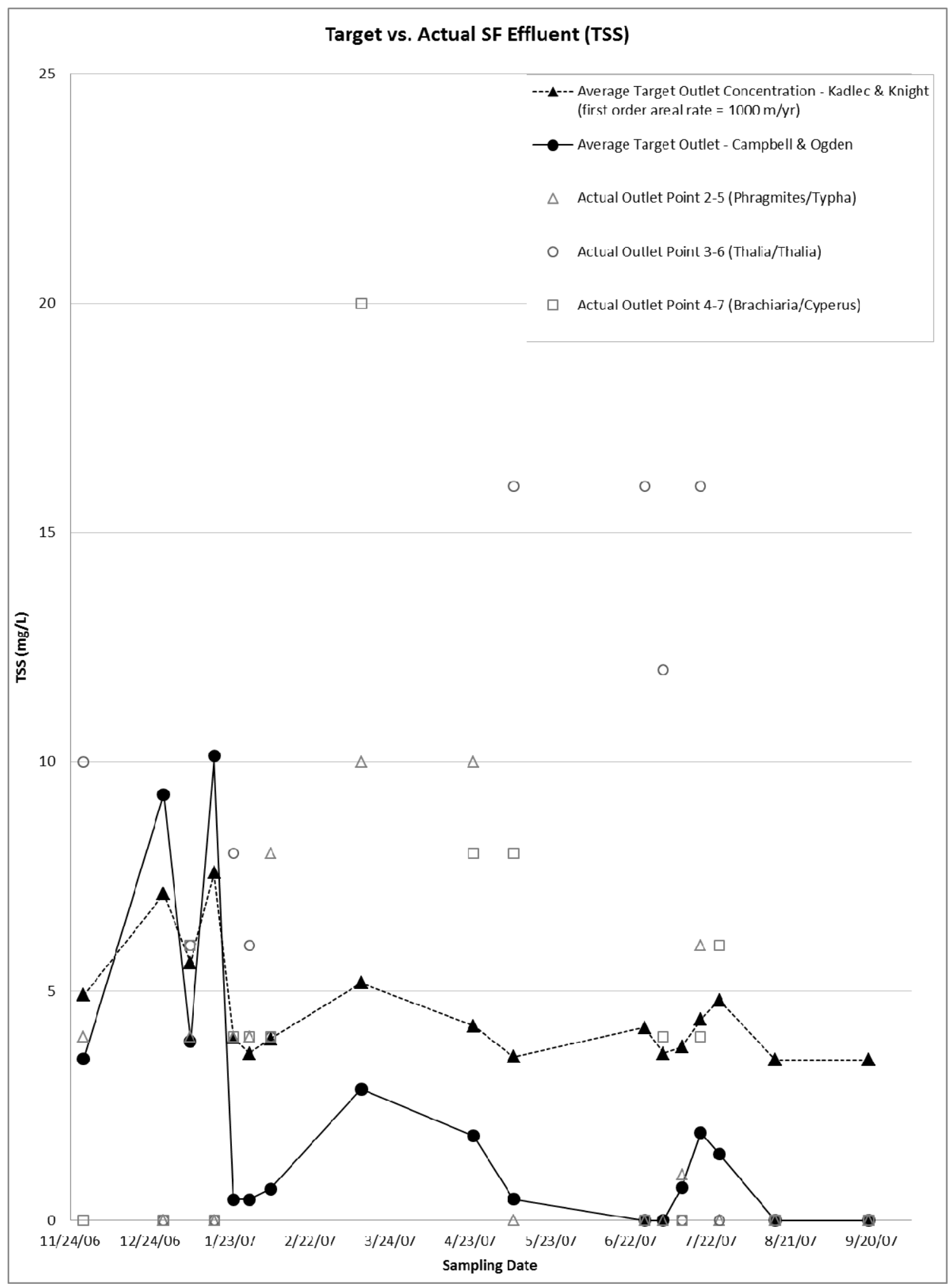

Figure 3-16: TSS Design Target vs. Actual Effluent Concentrations - Kadlec \& Knight, Campbell \& Ogden (Closer View) 


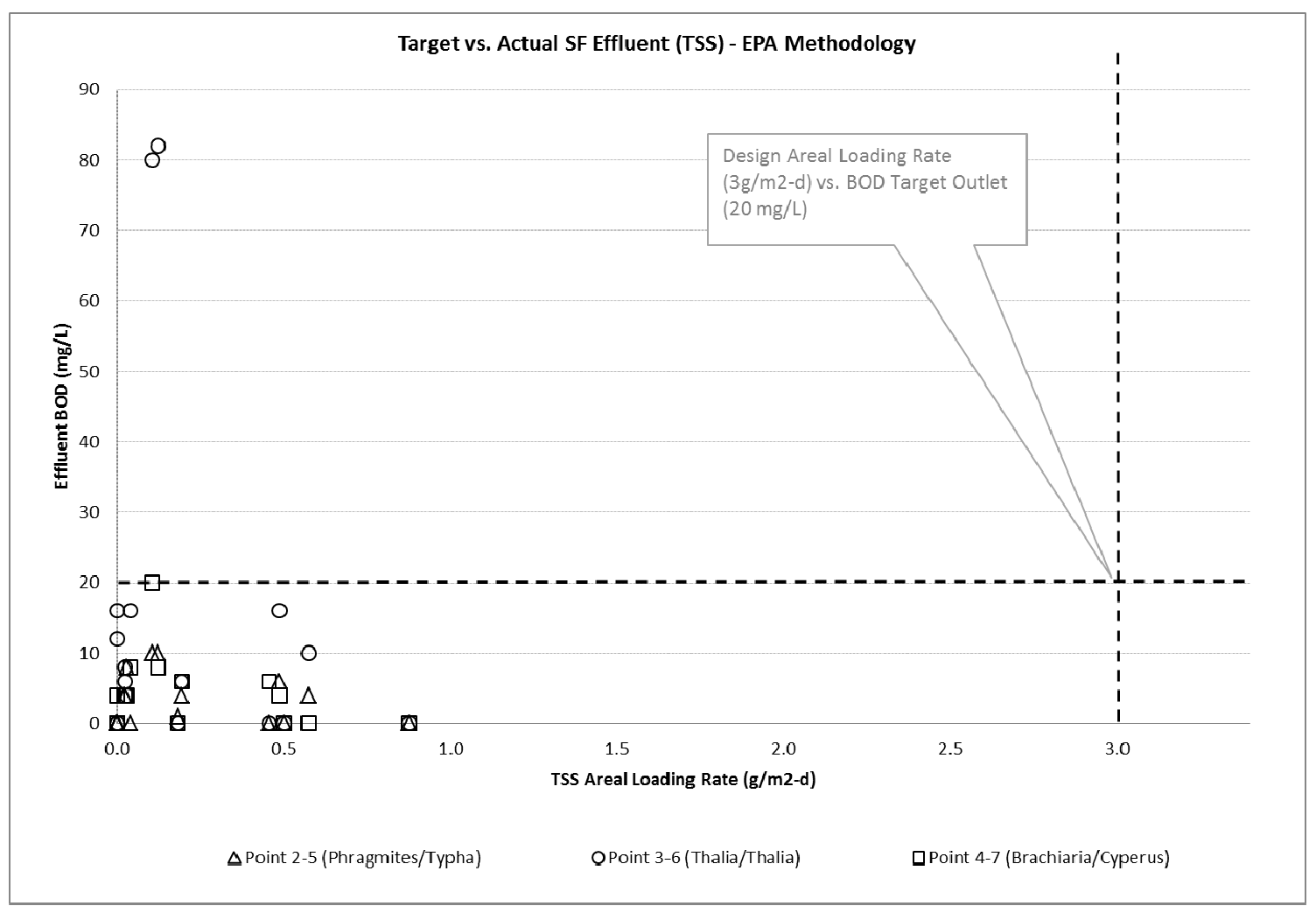

Figure 3-17: TSS Design Target vs. Actual Effluent Concentrations - EPA

\subsubsection{Total Nitrogen}

Kadlec \& Knight design calculation used a first-order areal rate constant (k) of $22 \mathrm{~m} / \mathrm{yr}$, but the actual average $\mathrm{k}$ for all the three cells was $8.5 \mathrm{~m} / \mathrm{yr} \pm 14.8 \mathrm{~m} / \mathrm{yr}$, considered not significantly different than the $\mathrm{k}$ used in the design. The Total Nitrogen background concentration used in the design calculation was $2 \mathrm{mg} / \mathrm{L}$, and the actual average background concentration was $4.1 \mathrm{mg} / \mathrm{L} \pm 0.3 \mathrm{mg} / \mathrm{L}$. As shown in Figure 3-18, the design Total Nitrogen target outlet (effluent) concentration based on the Kadlec \& Knight design values and actual SF dimensions, is somewhat different than the Total Nitrogen actual outlet (effluent) concentrations, but within an acceptable range. 
Based on the current model results presented in this Chapter, the Kadlec \& Knight methodology seems to have achieved results somewhat closer to the actual results.

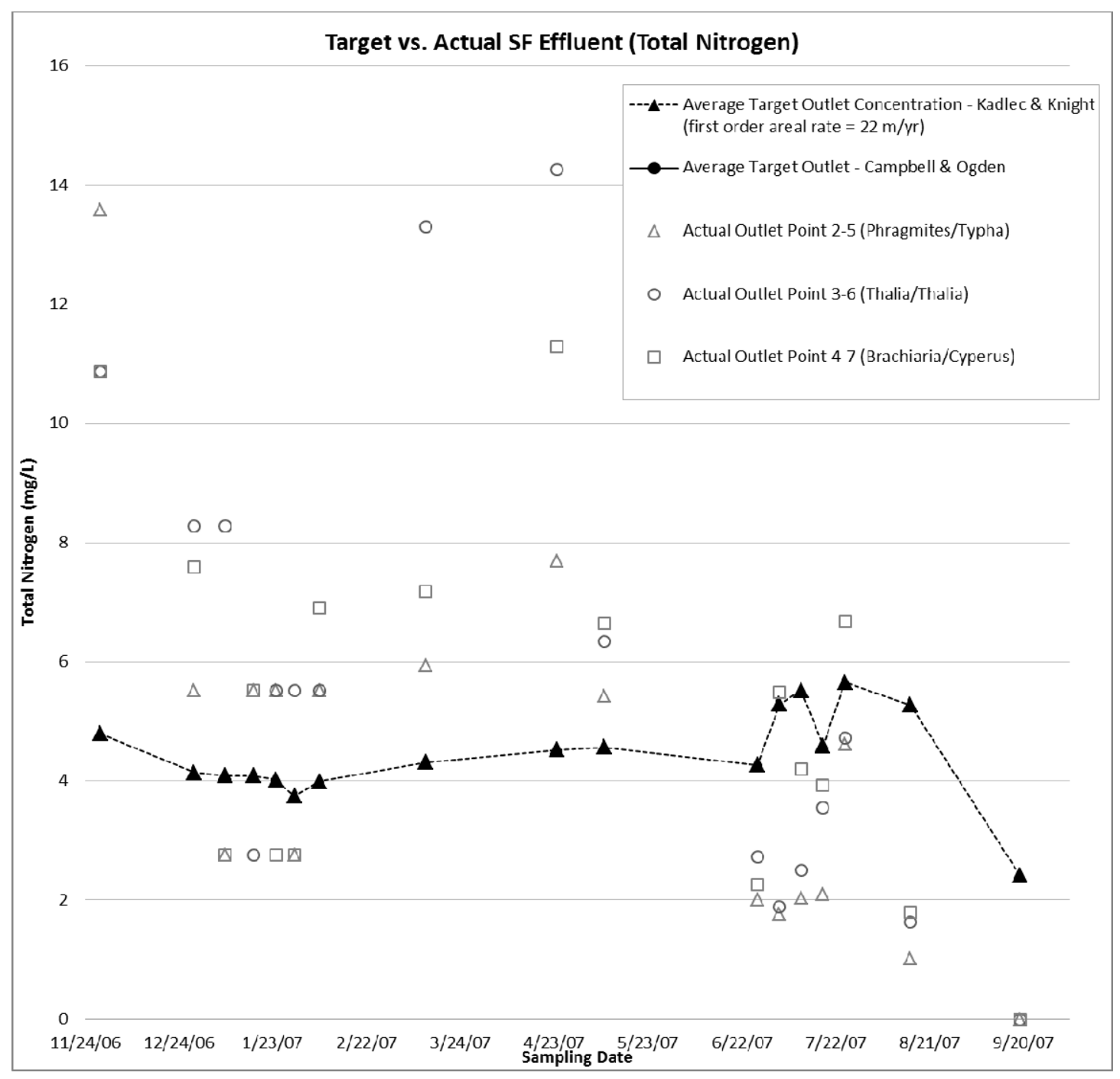

Figure 3-18: Total Nitrogen Design Target vs. Actual Effluent Concentrations - Kadlec \& Knight 


\subsection{Sensitivity Analysis}

Sensitivity Analysis was performed for BOD and TSS removals using the Kadlec model (as the Kadlec model was the model used for sizing the CW system of this study) to identify the parameters that have the greatest effect on the predicted results. The following parameters were analyzed for SSF and SF:

- First order areal rate constant;

- Background concentration; and

- Net flow.

The same methodology was used for both SSF and SF wetlands sensitivity analysis, as well as for both BOD and TSS, as described in the following chapters.

\subsubsection{Sensitivity Analysis for BOD}

First order areal rate constant, background concentration, and net flow values ranging from minimum and maximum were used in a set-interval for BOD, as presented in Table 3-17. The sensitivity analysis values were selected as the closest round numbers to the minimum and maximum values and the value used during design. 
Table 3-17: Sensitivity Analysis Minimum and Maximum Values Used for BOD

First-order areal rate constant $(\mathrm{k}$ in $\mathrm{m} / \mathrm{yr})$

\begin{tabular}{|c|c|c|c|c|c|c|c|c|c|}
\hline & $\begin{array}{l}\text { Point } \\
1-2\end{array}$ & $\begin{array}{l}\text { Point } \\
1-3\end{array}$ & $\begin{array}{l}\text { Point } \\
1-4\end{array}$ & $\begin{array}{l}\text { Point 1-2 } \\
\text { Point 1-3 } \\
\text { Point 1-4 }\end{array}$ & $\begin{array}{l}\text { Point } \\
2-5\end{array}$ & $\begin{array}{l}\text { Point } \\
3-6\end{array}$ & \multicolumn{2}{|l|}{$\begin{array}{l}\text { Point } \\
4-7\end{array}$} & $\begin{array}{l}\text { Point 2-5 } \\
\text { Point 3-6 } \\
\text { Point 4-7 }\end{array}$ \\
\hline Minimum & 2.5 & 0.6 & 12.0 & 0.6 & 0.3 & 0.6 & \multicolumn{2}{|l|}{-1.2} & -1.2 \\
\hline Average & 13.3 & 22.1 & 23.3 & 19.7 & 5.5 & 5.4 & \multicolumn{2}{|l|}{9.9} & 7.0 \\
\hline Maximum & 39.6 & 77.5 & 78.0 & 78.0 & 12.7 & 26.6 & \multicolumn{2}{|l|}{55.7} & 55.7 \\
\hline $\begin{array}{l}\text { Sensitivity } \\
\text { Analysis } \\
\text { Values }\end{array}$ & \multicolumn{4}{|c|}{$1,5,10,15,20,180$} & \multicolumn{5}{|c|}{$-1,2.5,5,10,15,20,25,50$} \\
\hline \multicolumn{6}{|c|}{ Background Concentration $\left(\mathrm{c}^{*}\right.$ in $\left.\mathrm{mg} / \mathrm{L}\right)$} & \multicolumn{4}{|c|}{ Net Flow (Qnet in $\mathrm{m}^{3} /$ day) } \\
\hline & $\begin{array}{l}\text { Point } \\
1\end{array}$ & $\begin{array}{l}\text { Point } \\
1-2\end{array}$ & $\begin{array}{l}\text { Point } \\
1-3\end{array}$ & $\begin{array}{l}\text { Point } \\
1-4\end{array}$ & $\begin{array}{l}\text { Average } \\
\text { Point 1-2 } \\
\text { Point 1-3 } \\
\text { Point 1-4 }\end{array}$ & $\begin{array}{l}\text { Point } \\
\text { Point } \\
\text { Point }\end{array}$ & & $\begin{array}{l}\text { Point } \\
\text { Point } \\
\text { Point }\end{array}$ & $\begin{array}{l}2-5 \\
3-6 \\
4-7\end{array}$ \\
\hline Minimum & 5.2 & 4.2 & 4.6 & 4.6 & 4.5 & 8.1 & & 3.4 & \\
\hline Average & 7.0 & 6.2 & 6.3 & 5.7 & 6.1 & 14.6 & & 14.4 & \\
\hline Maximum & 11.6 & 8.4 & 12.4 & 7.8 & 9.5 & 36.9 & & 41.4 & \\
\hline $\begin{array}{l}\text { Sensitivity } \\
\text { Analysis } \\
\text { Values }\end{array}$ & \multicolumn{5}{|c|}{$0,1,5,7.5,10,15,20$} & \multicolumn{4}{|c|}{$5,10,15,20,25,30,35,40$} \\
\hline
\end{tabular}

The first order areal rate has the most potential to influence the BOD predicted values, as shown in Figure 3-19. The net flow was the second parameter with potential to influence the BOD predicted values (Figure 3-20). As their values changed, they moved away from the actual values. On the other hand, background concentration showed no 
substantial differences from the actual versus predicted values (Figure 3-21), with the BOD predicted values within the actual values.

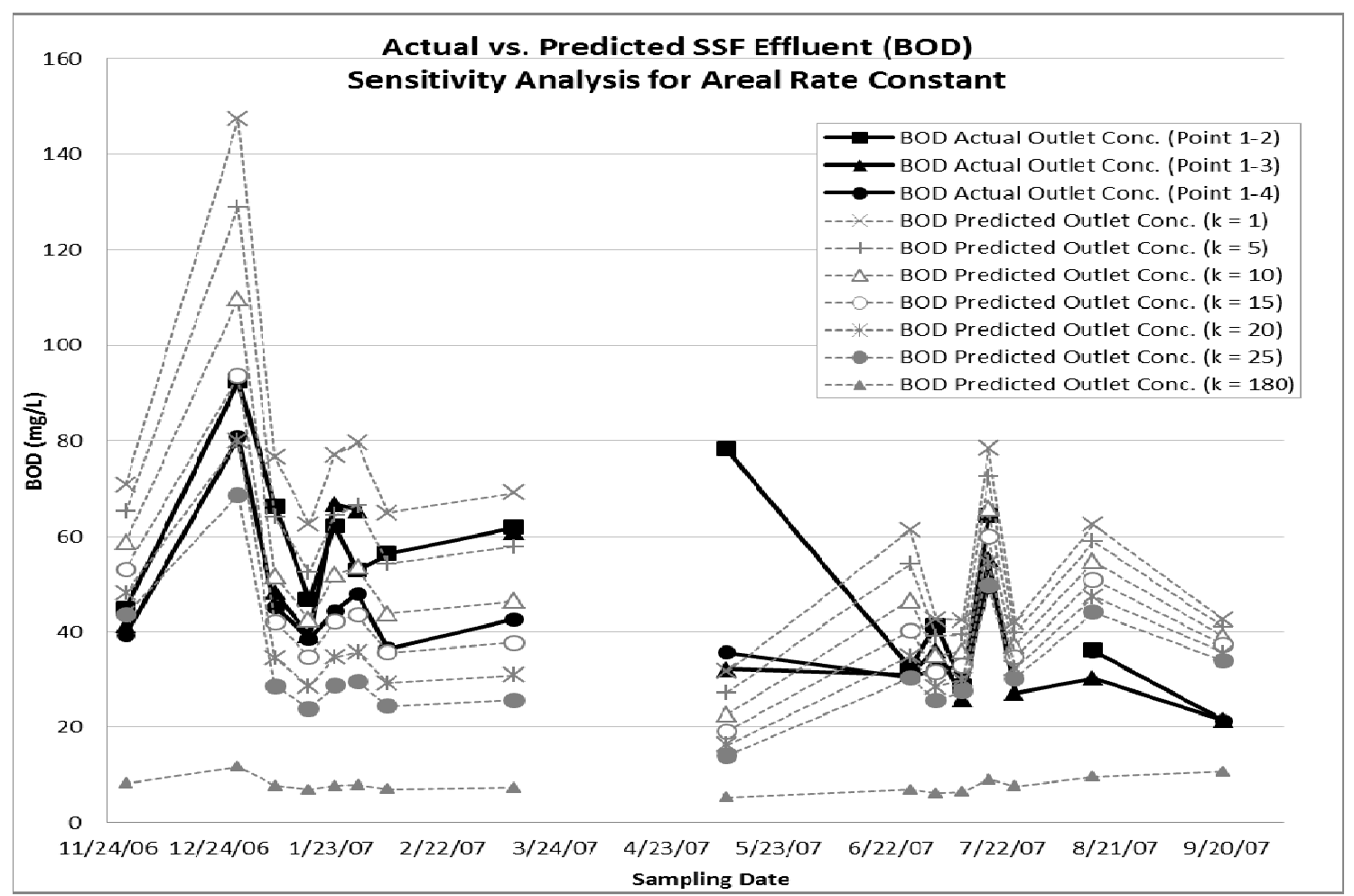

Figure 3-19: SSF Sensitivity Analysis for Areal Rate Constant (BOD) 


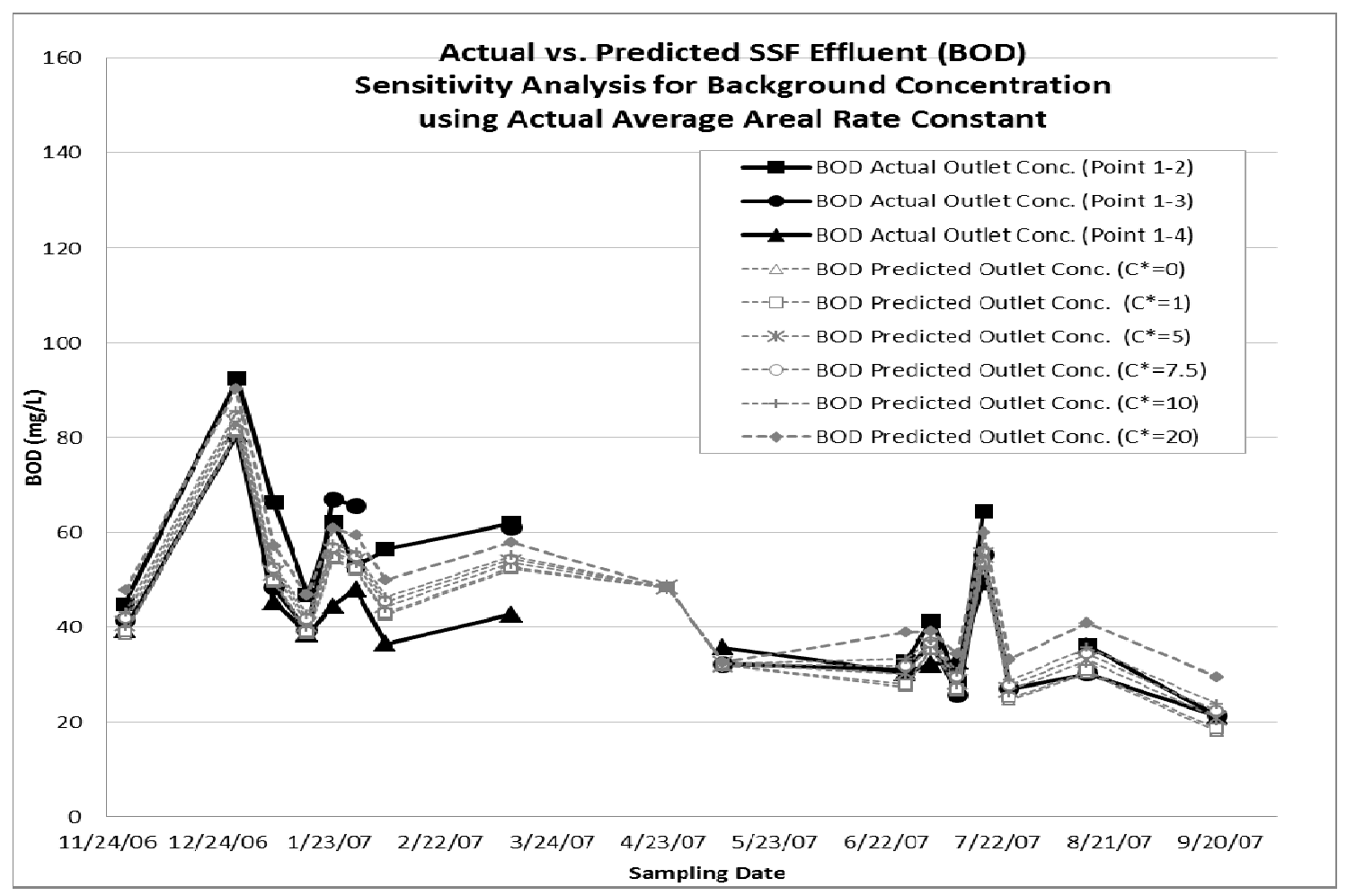

Figure 3-20: SSF Sensitivity Analysis for Net Flow (BOD)

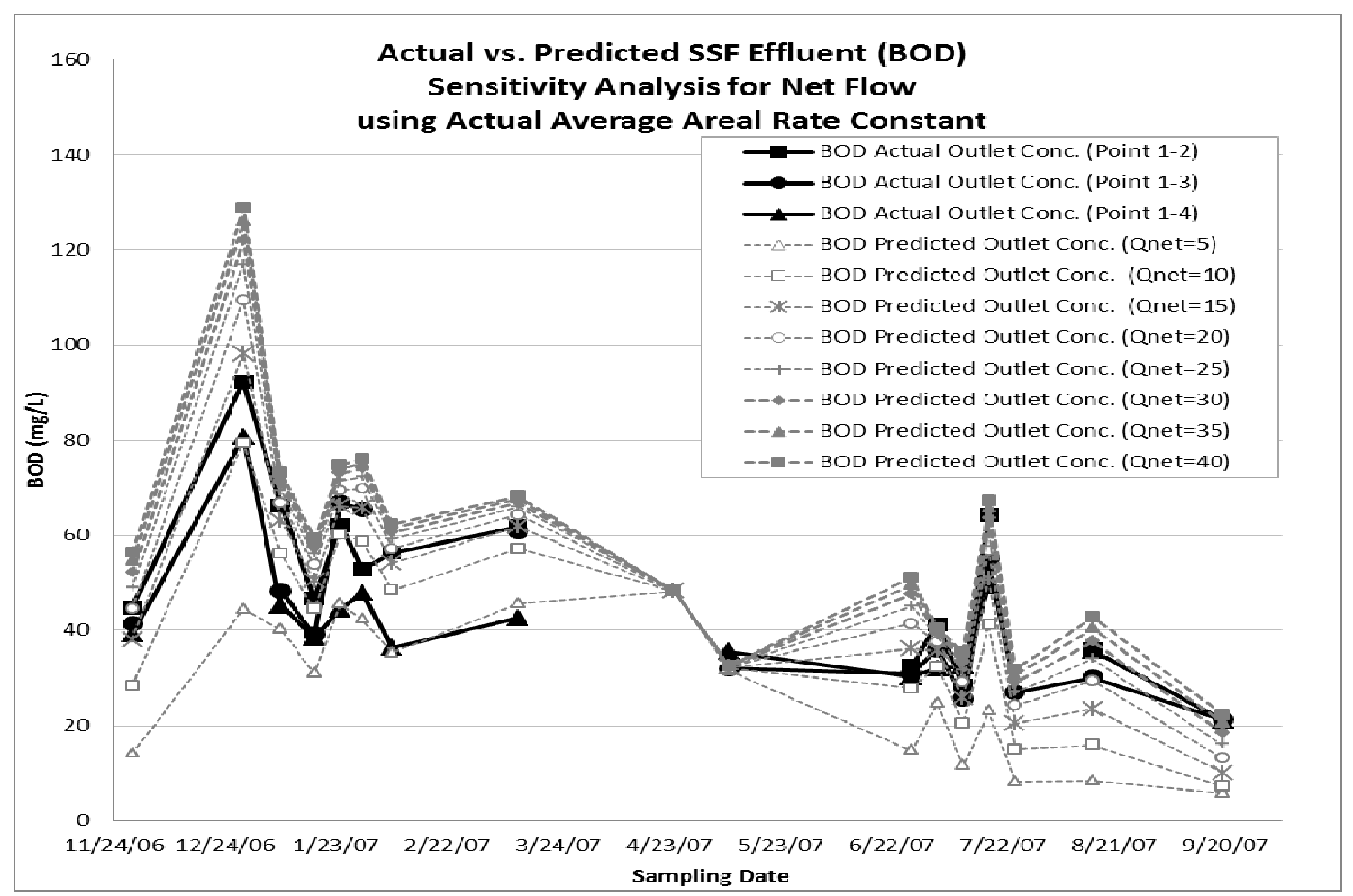

Figure 3-21: SSF Sensitivity Analysis for Background Concentration (BOD) 
Similar results were obtained for SF models for BOD, having the first areal rate constant as the most sensitive parameter. Sensitivity analysis results for BOD model predictions using SF wetland are presented in Appendix C.

\subsubsection{Sensitivity Analysis for TSS}

First order areal rate constant, background concentration, and net flow values ranging from minimum and maximum were used in a set-interval for TSS, as presented in Table 3-18. The sensitivity analysis values were selected as the closest round numbers to the minimum and maximum values and the value used during design.

TSS background concentration ( $\mathrm{c}^{*}$ ) had the most potential to influence the TSS predicted values, because when the $\mathrm{c}^{*}$ value is greater than the effluent TSS concentration, the areal rate constant $(\mathrm{k})$ for TSS could not be calculated, and the TSS effluent concentration could not be predicted (Figure 3-24). When the areal rate constant could be calculated, the net flow showed also potential to influence the TSS predicted values (Figure 3-23). As the background concentration and net flow values changed, the predicted values moved away from the actual values. The areal rate constant showed some potential to influence the TSS predicted values (Figure 3-22); however, it is important to note that the areal rate constant was not calculated using the actual background concentration; therefore, all the predicted values were calculated. 
Table 3-18: Sensitivity Analysis Minimum and Maximum Values Used for TSS

First-order areal rate constant $(\mathrm{k}$ in $\mathrm{m} / \mathrm{yr})$

\begin{tabular}{|c|c|c|c|c|c|c|c|c|c|}
\hline & $\begin{array}{l}\text { Point } \\
1-2\end{array}$ & $\begin{array}{l}\text { Point } \\
1-3\end{array}$ & $\begin{array}{l}\text { Point } \\
1-4\end{array}$ & $\begin{array}{l}\text { Point } 1-2 \\
\text { Point } 1-3 \\
\text { Point } 1-4\end{array}$ & $\begin{array}{l}\text { Point } \\
2-5\end{array}$ & $\begin{array}{l}\text { Point } \\
3-6\end{array}$ & \multicolumn{2}{|l|}{$\begin{array}{l}\text { Point } \\
4-7\end{array}$} & $\begin{array}{l}\text { Point 2-5 } \\
\text { Point 3-6 } \\
\text { Point 4-7 }\end{array}$ \\
\hline Minimum & 39.3 & 31.7 & 30.2 & 30.2 & -1.1 & -4.9 & \multicolumn{2}{|l|}{0.0} & -4.9 \\
\hline Average & 82.5 & 103.0 & 83.0 & 89.6 & 2.5 & 0.9 & \multicolumn{2}{|l|}{10.5} & 4.2 \\
\hline Maximum & 128.1 & 194.5 & 164.4 & 194.5 & 21.7 & 10.2 & \multicolumn{2}{|l|}{38.7} & 38.7 \\
\hline $\begin{array}{l}\text { Sensitivity } \\
\text { Analysis } \\
\text { Values }\end{array}$ & \multicolumn{4}{|c|}{$\begin{array}{l}30,60,90,120,150,180,210,500,1000 \\
1500\end{array}$} & \multicolumn{5}{|c|}{$-5,-1,5,10,15,20,25,50$} \\
\hline \multicolumn{6}{|c|}{ Background Concentration $\left(\mathrm{c}^{*}\right.$ in $\left.\mathrm{mg} / \mathrm{L}\right)$} & \multicolumn{4}{|c|}{ Net Flow (Qnet in $\mathrm{m}^{3} /$ day) } \\
\hline & $\begin{array}{l}\text { Point } \\
1\end{array}$ & $\begin{array}{l}\text { Point } \\
1-2\end{array}$ & $\begin{array}{l}\text { Point } \\
1-3\end{array}$ & $\begin{array}{l}\text { Point } \\
1-4\end{array}$ & $\begin{array}{l}\text { Average } \\
\text { Point 1-2 } \\
\text { Point 1-3 } \\
\text { Point 1-4 }\end{array}$ & $\begin{array}{l}\text { Point } \\
\text { Point } \\
\text { Point }\end{array}$ & & $\begin{array}{l}\text { Point } \\
\text { Point } \\
\text { Point }\end{array}$ & $\begin{array}{l}2-5 \\
3-6 \\
4-7\end{array}$ \\
\hline Minimum & 9.4 & 3.5 & 3.5 & 3.5 & 3.5 & 8.1 & & 3.4 & \\
\hline Average & 18.0 & 4.5 & 4.7 & 4.5 & 4.6 & 13.2 & & 14.4 & \\
\hline Maximum & 47.5 & 8.2 & 9.0 & 5.8 & 7.6 & 25.0 & & 41.4 & \\
\hline $\begin{array}{l}\text { Sensitivity } \\
\text { Analysis } \\
\text { Values }\end{array}$ & \multicolumn{5}{|c|}{$\begin{array}{l}0,10,20,30,40,50,100 \text { (for Point } 1 \text { ) } \\
0,1,5,7.5,10,15,20 \text { (for Point 1-2, Point 1-3, 1-4) }\end{array}$} & \multicolumn{4}{|c|}{$5,10,15,20,25,30,35,40$} \\
\hline
\end{tabular}




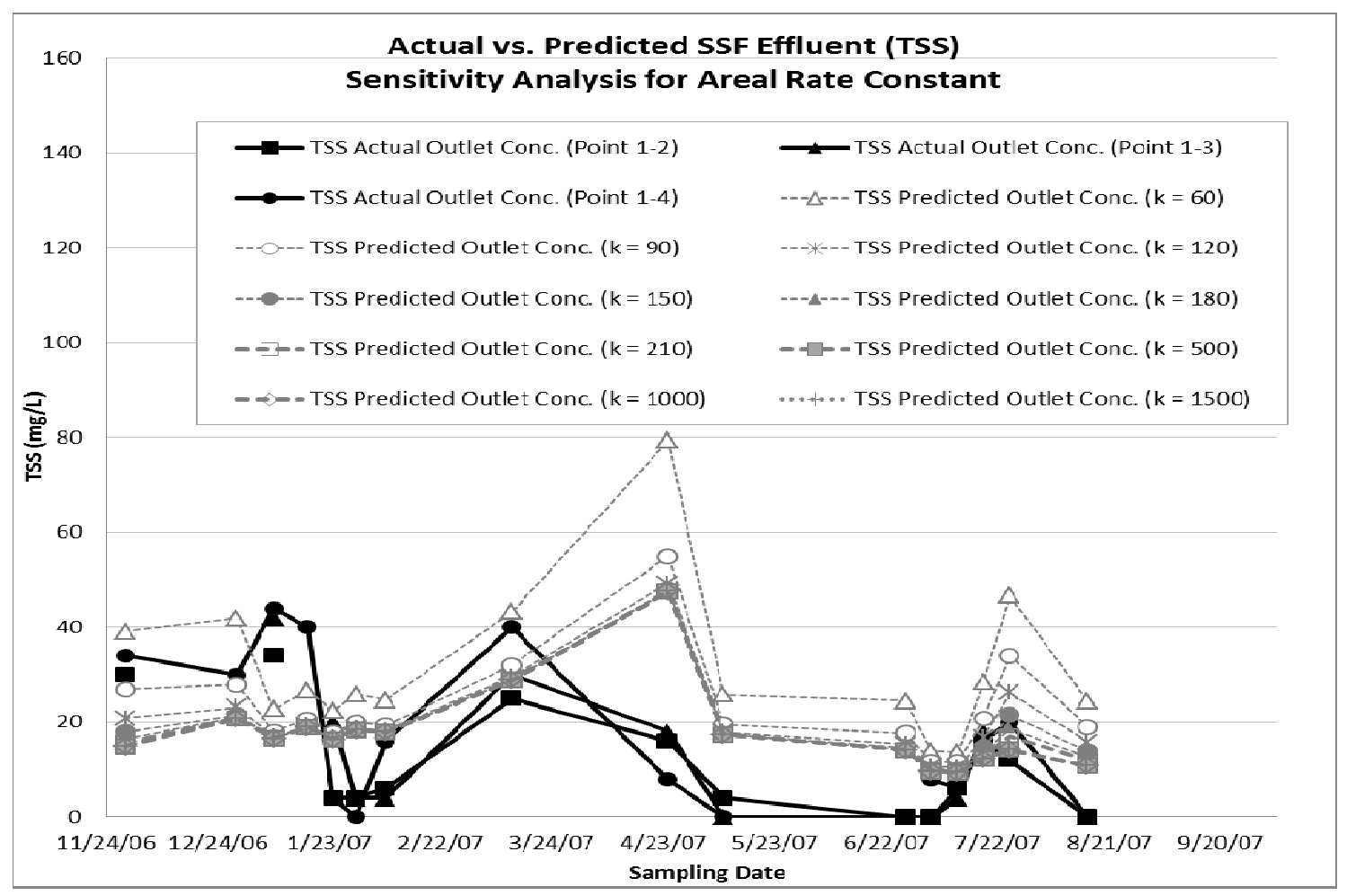

Figure 3-22: SSF Sensitivity Analysis for Areal Rate Constant (TSS)

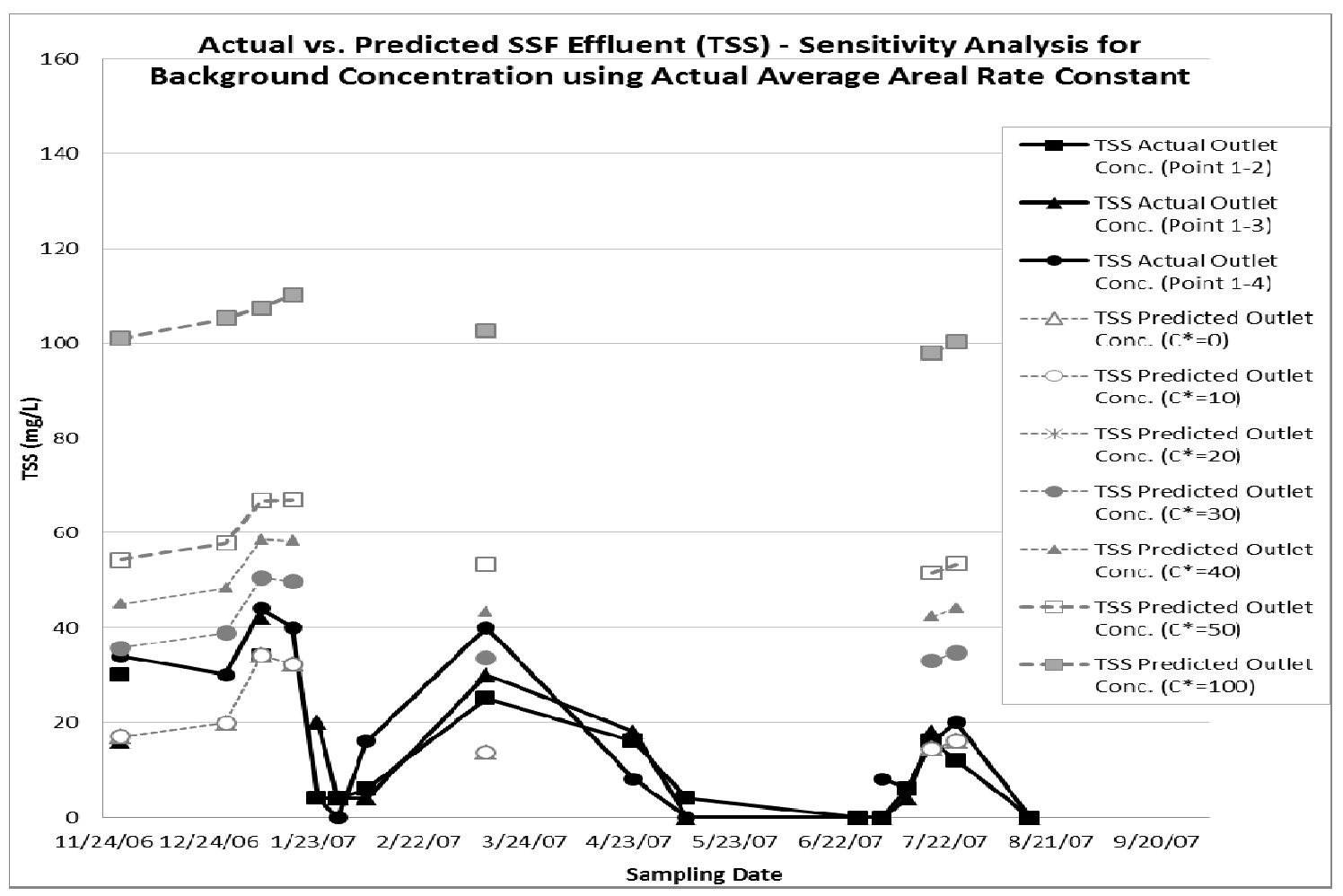

Figure 3-23: SSF Sensitivity Analysis for Net Flow (TSS) 


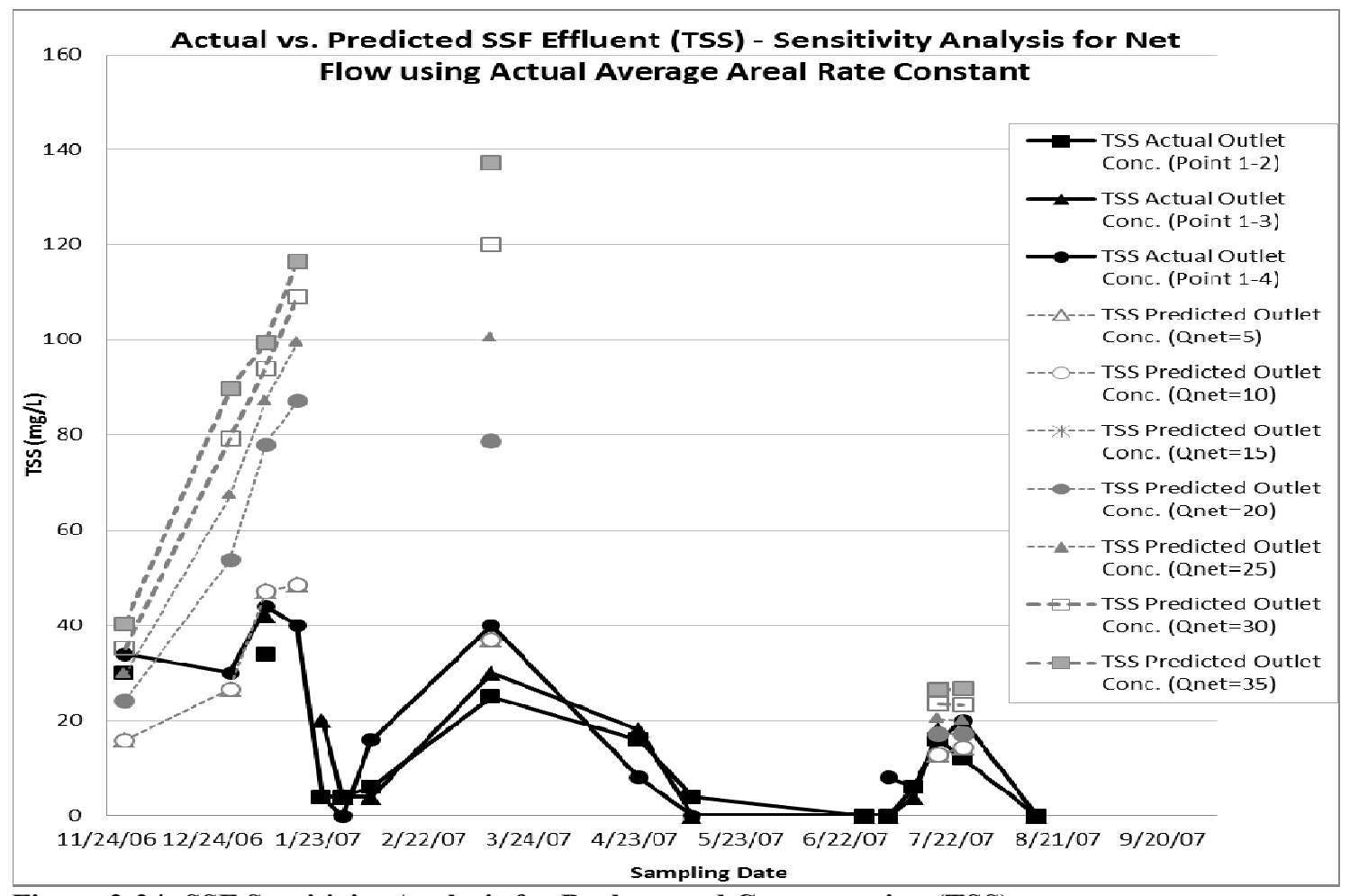

Figure 3-24: SSF Sensitivity Analysis for Background Concentration (TSS)

Similar results were obtained for SF models for TSS, having the TSS background concentration as the most sensitive parameter. Sensitivity analysis results for TSS model predictions using SF wetland are presented in Appendix C. 


\section{CONCLUSIONS, LIMITATIONS AND RECOMMENDATIONS}

\subsection{Conclusions}

The central hypothesis of this research was "that the constructed wetland system of the study is capable to provide competitive secondary treatment levels, removing significant amounts of biological oxygen demand $\left(\mathrm{BOD}_{5}\right)$, total suspended solids (TSS) and pathogens among other water quality indicators, under tropical climatic conditions." Consequently to the detailed discussion of the measured water quality parameters (Chapters 2 and 3), the results strongly suggest that this CW system can effectively reduce contaminants in wastewater to levels that are comparable with the objective levels (i.e., secondary treatment levels). The $\mathrm{CW}$ system performance also proved to be reliable under the extremes of tropical conditions, such as those occurring during the peaks of the wet and dry seasons in El Salvador. Conclusively, the central hypothesis of the research was proven to be correct.

Based on the results of organic and nutrients removal analyses, Phragmites and Brachiaria were the most effective SSF plants; Brachiaria provided the added benefit of serving as a source of fodder. Typha produced the best dry season results within the SF component. Monitoring Point 1-2-5 (Inlet/Phragmites/Typha) performed better yearround than either Monitoring Points 1-3-6 or 1-4-7 (Inlet/Thalia/Thalia or Inlet/Brachiaria/Cyperus, respectively). Similarly, Monitoring Point 1-4-7 reduced contaminant concentrations more than did Monitoring Point 1-3-6 over both seasons. Conditions were only slightly more conducive to reduction over the wet season, although this effect becomes much more exaggerated when the effects of evapotranspiration and 
dilution are considered. Amongst all measured parameters, the greatest reductions were observed with Total Nitrogen, TSS and Fecal coliform, perhaps due to such efficient reduction in ammonium and physical processes as sedimentation and filtration (Songa, et al., 2006).

All the research objectives were also achieved, as follows:

1) The design approach of the field scale SSF CW was described in Chapter 2.1.3.

2) The SSF characterization of influent and effluents for $\mathrm{BOD}_{5}, \mathrm{COD}$, TSS, Oil \& Grease, Fecal Coliform, Total Nitrogen, and Phosphorus was presented in Chapter 3.1 .

3) Comparative assessment of empirical models and selection of most appropriate models used during design for $\mathrm{BOD}_{5}$, TSS, and Total Nitrogen were presented in Chapter 3.2, and it was concluded that:

a) The EPA Methodology seems to have achieved more realistic results than the other methodologies for $\mathrm{BOD}_{5}$ removal;

b) All evaluated methodologies seem to have achieved results somewhat closer to the actual TSS results; and

c) Kadlec \& Knight methodology for Total Nitrogen removal seems to have achieved results somewhat closer to the actual results.

4) Comparison of the differences in treatment associated with each one of the selected plants was described in Chapter 3.1. 


\subsection{Limitations and Recommendations}

Based on the constructed wetland system performance, the following is recommended:

- The flow in this study was poorly operated and lacked in a trustful flow. In order to improve the flow condition, one of the cells could have been closed down during the dry season to increase the flow and have a more constant flow throughout the year, which would result in a more constant performance. However, the only drawback of closing down one of the cells is that the plants in that cell would require an irrigation system to keep the plants alive until the wet season.

- For future studies, building a small reactor prior to the actual pilot study in order to simulate the tropical climate conditions is recommended to minimize the difference between the actual and the predicted results.

- More monitoring would allow for better results, and the seasonal variation would be even more obvious. Daily monitoring would be ideal; but for budget limitations, once a week would be the least frequency recommended.

- The relationships between inflow and outflow loadings, instead of concentrations, may be a better approach for comparison of results during different seasons, as the flow greatly varied from dry and wet seasons due to the difference in precipitation.

- Future designs should consider a reduction in the facultative lagoon total area and incorporate measures that optimize resilience and responsiveness to dynamic 
water flow patterns over the course of the year. SSF CW should be used cautiously to treat facultative lagoon effluents due to algae bloom, the leading cause of SSF CW clogging. Care should be taken to prevent the discharge of military-specific pollutants during the dry season when system equalization abilities are limited.

- The depth of OW Sections should be further evaluated in order to minimize limited water mixing and stagnant conditions at the bottom of OW sections,.

- Even though Cyperus showed a slightly better performance for $\mathrm{BOD}_{5}$ removal in the SF CW than Typha and Thalia; Cyperus required high maintenance which deviates from the purpose of $\mathrm{CW}$ systems of minimal maintenance requirements. Therefore, Cyperus would not be recommended for SF CWs.

- The measured plant biomass $\mathrm{N}$ and $\mathrm{P}$ content is comparable to other studies of constructed wetlands (Kadlec \& Wallace, 2008); however, further research is needed to examine trends in vegetation nutrients content in dry and wet seasons. 


\section{REFERENCES}

Akratos, C. S. \& Tsihrintzis, V. A., 2007. Effect of temperature, HRT, vegetation and porous media on removal efficiency of pilot-scale horizontal subsurface flow constructed wetlands. Ecological Engineering, 29(2), pp. 173-191.

Bojcevska, H. \& Tonderski, K., 2007. Impact of loads, season, and plant species on the performance of a tropical constructed wetland polishing effluent from sugar factory stabilization ponds. Ecol. Eng., pp. 66-76.

Brisson, J. \& Chazarenc, F., 2009. Maximizing pollutant removal in constructed wetlands: should we pay more attention to macrophyte species selection?. Science of the Total Environment, 407(13), pp. 3923-3930.

Brix, H., 1997. Do macrophytes play a role in constructed wetlands?. Water Sci. Technol., 35(5), pp. 11-17.

Brix, H. \& Arias, A. C., 2005. The use of vertical flow constructed wetlands for on-site treatment of domestic wastewater: New Danish guidelines. Ecol. Eng., Volume 25, pp. $491-500$.

Burks, B. D. \& Minnis, M. M., 1994. Onsite Wastewater Treatment Systems. Madison, WI: Hogarth House, Ltd.

Campbell, C. S. \& Ogden, M., 1999. Constructed Wetlands in the Sustainable Landscape.. s.1.:John Wiley \& Sons, Inc..

Caselles-Osorio, A. et al., 2007. Solids accumulation in six full-scale subsurface flow constructed wetlands. Water Research, Volume 41, pp. 1388-1398.

Caselles-Osorio, A., Villafane, P., Caballero, V. \& Manzano, Y., 2011. Efficiency of Mesocosm-Scale Constructed Wetland Systems for Treatment of Sanitary Wastewater Under Tropical Conditions. Water Air Soil Pollut, Volume 220, pp. 161-171.

CH2M Hill and Payne Engineering, 1997. Treatment Wetlands for Livestock Wastewater Management Literature Review, Database, and Research Synthesis., s.l.: Prepared for the Gulf of Mexico Program, Nutrient Enrichment Committee. Prepared under contract to National council of the Paper Industry for Air and Stream Improvement (NCASI) and Alabama Soil and Water conservation Committee.

Cooper, D. J., Griffin, P. \& Cooper, P. F., 2005. Factors affecting the longevity of subsurface horizontal flow systems operating as tertiary treatment for sewage efluent. Water Science and Technology, Volume 51, pp. 127-135. 
Crites, R. a. G. T., 1998. Small and Decentralized Wastewater Management Systems. s.l.:s.n.

Department of the Army, the Navy, and the Air Force, 1988. Domestic Wastewater. ARMY TM 5-814-3, Air Force AFM 88-11.Vol 3., s.1.: s.n.

ESTCP, 1999. The Use of Constructed Wetlands to Phytoremediate ExplosivesContaminated Groundwater at the Milan Army Ammunition Plant, Milan, Tennessee: s.n.

Green, M., Friedler, E. \& Safrai, I., 1998. Enhancing nitrification in vertical flow constructed wetland utilizing a passive air pump. Water Res., 32(12), pp. 3513-3520.

Gross, A., Kaplan, D. \& Baker, K. H., 2007. Rmoval of chemical and microbiological contaminants from domestic greywater using a recirculating vertical flow bioreactor. Ecol. Eng., 31(2), pp. 107-114.

Halverson, N. V., 2004. Review of Constructed Subsurface Flow vs. Surface Flow Wetlands, s.1.: U.S. Department of Energy. WSRD-TR-2004-00509.

Headquarters, Department of the Army, 1987. Technical Manual, Evaluation Criteria Guide for Water Pollution Prevention, Control, and Abatement Programs, s.1.: TM 5814-8.23.

Hijosa-Valsero, M., Sidrach-Cardona, R., Martin-Villacorta, J. \& Becares, E., 2010. Optimization of performance assessment and design characteristics in constructed wetlands for the removal of organic matter. Chemosphere, Volume 81, pp. 651-657.

Johnston, C. A., 1991. Sediments and nutrient retention by freshwater wetlands: effects on surface water quality.. CRC Critical Reviews in Environmental Control, Volume 21, pp. 491-565.

Kadlec, R. H. \& Knight, R. L., 1996. Treatment Wetlands. Boca Raton, FL: CRC Press LLC.

Kadlec, R. H. et al., 2000. Treatment wetlands for pollution control: processes, performance, design and operation. London, UK: IWA Publishing.

Kadlec, R. H. \& Wallace, S. D., 2008. Treatment Wetlands. Second Edition ed. Boca Raton, FL.: CRC Press.

Kantawanichkul, S., Somprasert, S., Aekasin, U. \& Shutes, R. E., 2003. Treatment of agricultural wastewater in two experimental combined constructed wetland systems in tropical climate. Water Sci. Technol., 48(5), pp. 199-205. 
Kaseva, M. E., 2004. Performance of a Sub-surface flow constructed wetland in polishing pre-treated wastewater-a tropical case study. Water Res., pp. 681-687.

Katsenovich, Y. P., Hummel-Batista, A., Ravinet, A. J. \& Miller, J. F., 2009. Performance Evaluation of Constructed Wetlands in a Tropical Region. Ecol Engineering, Volume 35, pp. 1529-1537.

Kentula, M. E., 1999. Restoration, Creation, and Recovery of Wetlands: National Water Summary on Wetland Resources, s.1.: United States Geological Survey Water Supply Paper 2425.

Kiker, J. H., Larson, S., Moses, D. D. \& Sellers, R., 2001. Use of Engineered Wetlands to Phytoremediate Explosives Contaminated Surface Water at the Iowa Army Ammunition Plant, Middletown, Iowa. s.1., s.n.

Knowles, P., Nivala, J., Dotro, J. \& Garcia, G., 2011. Clogging in subsurface-flow treatment wetlands: occurrence and contributing factors. Ecological Engineering, Volume 37, pp. 99-112.

Lahav, O., Artzi, E., Tarre, S. \& Green, M., 2001. Ammonium removal using a novel unsaturated flow biological filter with passive aeration. Water Res., 35(2), pp. 397-404.

Lazareva, O. \& Pichler, T., 2010. Long-term performance of constructed wetland/filter basin system treating wastewater, Central Florida. Chemical Geology, Volume 269, pp. 137-152.

Lorion, R., 2001. Constructed Wetlands: Passive Systems for Wastewater Treatment, s.1.: USEPA Technology Innovation Office.

Maloszewski, P., Wachniew, P. \& Czuprynski, P., 2006. Study of hydraulic parameters in heterogeneous gravel beds: Constructed wetland in Nowa Siupia (Poland). Journal of Hydrology, Volume 331, pp. 630-642.

Mara, D., 2004. To plant or not to plant? Questions on the role of plants in constructed wetlands, in: Wetland Systems and Waste Stabilization Ponds, Pro.. Avignon, France, 9th IWA International Conference on Wetland Systems.

Marsili-Libelli, S. \& Checchi, N., 2005. Identification of dynamic models for horizontal subsurface constructed wetlands.. Ecological Modelling, Volume 187, pp. 201-218.

Mashauri, D. A. \& Kayombo, S., 2002. Application of the two coupled models for water quality management: facultative pond cum constructed wetland models.. Physics and Chemistry of the Earth, Volume 27, pp. 773-781. 
Mburu, N. et al., 2013. Performance Evaluation of Horizontal Subsurface FlowConstructed Wetlands for the Treatment of Domestic Wastewater in the Tropics. Journal of Environmental Engineering, Issue March 2013, pp. 358-367.

McCormick, N. G., Cornell, J. H. \& Kaplan, A. M., 1984. The Fate of Hexahydro-1,3,5trinitro-1,3,5-triazine (RDX) and Related Compounds in Anaerobic Denitrifying Continuous Culture Systems Using Simulated Wastewater, s.1.: U.S. Army Natick Research \& Development Center, Tech. Rep. Natick/TR-85/008.

Naval Facilities Engineering Service Center (NFESC), 1998. Constructed Wetlands: Cost effective treatment of non-point source pollution, s.l.: NFESC TechData Sheet. TDS2034-ENV..

Pedescoll, A. et al., 2011. The effect of primary treatment and flow regime on clogging development in horizontal subsurface flow constructed wetlands: An experimental evaluation. Water Research, Volume 45, pp. 3579-3589.

Reed, S. C., Crites, R. W. \& Middlebrooks, E. J., 1995. Natural Systems for Waste Management and Treatment (2 ed.). USA: McGraw Hill, Inc..

Rodgers, J. H. et al., 2002. Treatment Wetlands: A Low Cost Solution for Storm Water and Point Source Wastewater Treatment at Savannah River Site, South Carolina: SCEC.

Rousseau, D. L., Horton, D., Vanrolleghem, P. A. \& De Pauw, N., 2005. Impact of operational maintenance on the asset life of storm reed beds. Water Science and Technology, Volume 51, pp. 243-250.

Rousseau, D. L., Vanrolleghem, P. A. \& De Pauw, N., 2004. Model-based design of horizontal subsurface flow constructed treatment wetlands: a review. Water Research, 38(6), pp. 1484-1493.

Saeed, T. \& Sun, G., 2011. Kinetic modelling of nitrogen and organics removal in vertical and horizontal flow wetlands. Water Research, Volume 45, pp. 3137-3151.

Schueler, T. A. R. T., 1992. Design of Stormwater Wetland Systems - Guidelines for Creating Diverse and Effective Stormwater Wetland Systems in the Mid-Atlantic Region., Washington, D.C.: Metropolitan Washington Council of Governments..

Sikora, F. J. et al., 1997. A Microcosm Study on Remediation of Explosives Contaminated Groundwater Using Treatment Wetlands. Bioremediation of Surface and Subsurface Contamination, s.l.: Annals of the New York Academy of Sciences, Vol. 829. 
Sklarz, M. Y., Gross, A., Soares, M. I. M. \& Yakirevich, A., 2010. Mathematical model for analysis of recirculating vertical flow constructed wetlands. Water Research, Volume 44, pp. 2010-2020.

Sklarz, M. Y., Gross, A., Yakirevich, A. \& Soares, M. M., 2009. A recirculating vertical flow constructed wetland for the treatment of domestic wastewater. Desalination, Volume 246, pp. 617-624.

Songa, Z. et al., 2006. Seasonal and annual performance of a full-scale constructed wetland system for sewage treatment in China.. Ecol. Eng., Volume 26, p. 272-282.

Stefanakis, A. I. \& Tsihrintzis, V. A., 2009. Performance of pilot-scale vertical flow constructed wetlands treating simulated municipal wastewater: effect of various design parameters. Desalination, Volume 248, pp. 753-770.

Stefanakis, A. I. \& Tsihrintzis, V. A., 2012. Effects of loading, resting period, temperature, porous media, vegetation and aeration on performance of pilot-scale vertical flow constructed wetlands. Chemical Engineering Journal, Volume 181-182, pp. 416430 .

Tan, K., Jacksona, W. A., Anderson, T. A. \& Pardue, J. H., 2004. Fate of perchloratecontaminated water in upflow wetlands. Water Research, pp. 4173-4185.

Trang, N. T. D. et al., 2010. Kinetics of pollutant removal from domestic wastewater in a tropical horizontal subsurface flow constructed wetland system: Effects of hydraulic loading rate. Ecological Engineering, Volume 36, pp. 527-535.

Tsihrintzis, V. A. et al., 2007. Performance and Cost Comparison of a FWS and a VSF Constructed Wetland System. Environmental Technology, Volume 28, pp. 621-628.

Tuncsiper, B., 2009. Nitrogen in a combined vertical and horizontal subsurface-flow constructed wetland system. Desalination, Volume 247, pp. 466-475.

U.S. Army Corps of Engineers, 2003. Applicability of Treatment Wetlands for Army Installations, Washington, D.C.: Public Works Technical Bulletin 200-1-21.

U.S. Department of Agriculture (USDA), Natural Resources Conservation Service (NRCS), 1991. Treatment Wetlands for Agricultural Waste Treatment, Technical Requirements, Washington, D.C.: SCS.

U.S. Department of Defense, 1999. The Use of Treatment Wetlands to Phytoremediate Explosives Contaminated; Groundwater at the Milan Army Ammunition Plant, Milan, Tennessee, s.1.: Environmental Security Technology Certification Group. 
U.S. EPA, 1980. Design Manual - Onsite Wastewater Treatment and Disposal Systems, s.1.: EPA 625/1-80-012.

U.S. EPA, 1999. Manual, Treatment Wetland Treatment of Municipal Wastewater, s.1.: EPA/625/R-99/010.

Vymazal, J., 2004. Removal of phosphorus in constructed wetlands with sub-surface flow in the Czech Republic.. Water, Air, and Soil Pollution, Volume 4, pp. 657-670.

Vymazal, J., 2005. Horizontal sub-surface flow and hybrid constructed systems for wastewater treatment. Ecol. Eng., Volume 25, pp. 478-490.

Vymazal, J., 2007. Removal of nutrients in various types of constructed wetlands. Sci. Total Environ., Volume 380, pp. 48-65.

Vymazal, J., 2011. Constructed Wetlands for Wastewater Treatment: Five Decades of Experience. Environ. Sci. Technol, Volume 18, pp. 842-856.

Vymazal, J., 2011. Plants used in constructed wetlands with horizontal subsurface flow: a review. Hydrobiologia, Volume 674, pp. 133-156.

Vymazal, J., Dusek, J. \& Kvet, J., 1999. Nutrient uptake and storage by plants in constructed wetlands with horizontal sub-surface flow: a comparative study. Leiden: Backhuys Publishers.

Vymazal, J. \& Kropfelova, L., 2008. Wastewater Treatment in Constructed Wetlands with Horizontal Sub-Surface Flow.. Springer, Dordrecht: s.n.

Vymazal, J. \& Kropfelova, L., 2009. Removal of organics in constructed wetlands with horizontal sub-surface flow: A review of the field experience. Science of the Total Environment, Volume 407, pp. 3911-3922.

Wallace, S. D. \& Knight, R. L., 2006. Small-scale Constructed Wetland Treatment Systems. In: Feasibility, Design Criteria and O\&M Requirements. Final Report, Project 01-CTS-5. Alexandria, USA: Water Environment Research Foundation (WERF).

WEF, 2000. Design of Municipal Wastewater Treatment Plants, Manual of Practice Report No. 76, Volume I, Washington, USA: Water Environment Federation publishers.

Wynn, T. M. \& Liehr, S. K., 2001. Development of a constructed subsurface-flow wetland simulation model. Ecological Engineering, 16(4), pp. 519-536. 
Zhi, W. \& Ji, G., 2012. Constructed wetlands, 1991-2011: A review of research development, current trends, and future directions. Science of the Total Environment, Volume 441, pp. 19-27. 
APPENDICES

APPENDIX A

PLANS AND SECTIONS 


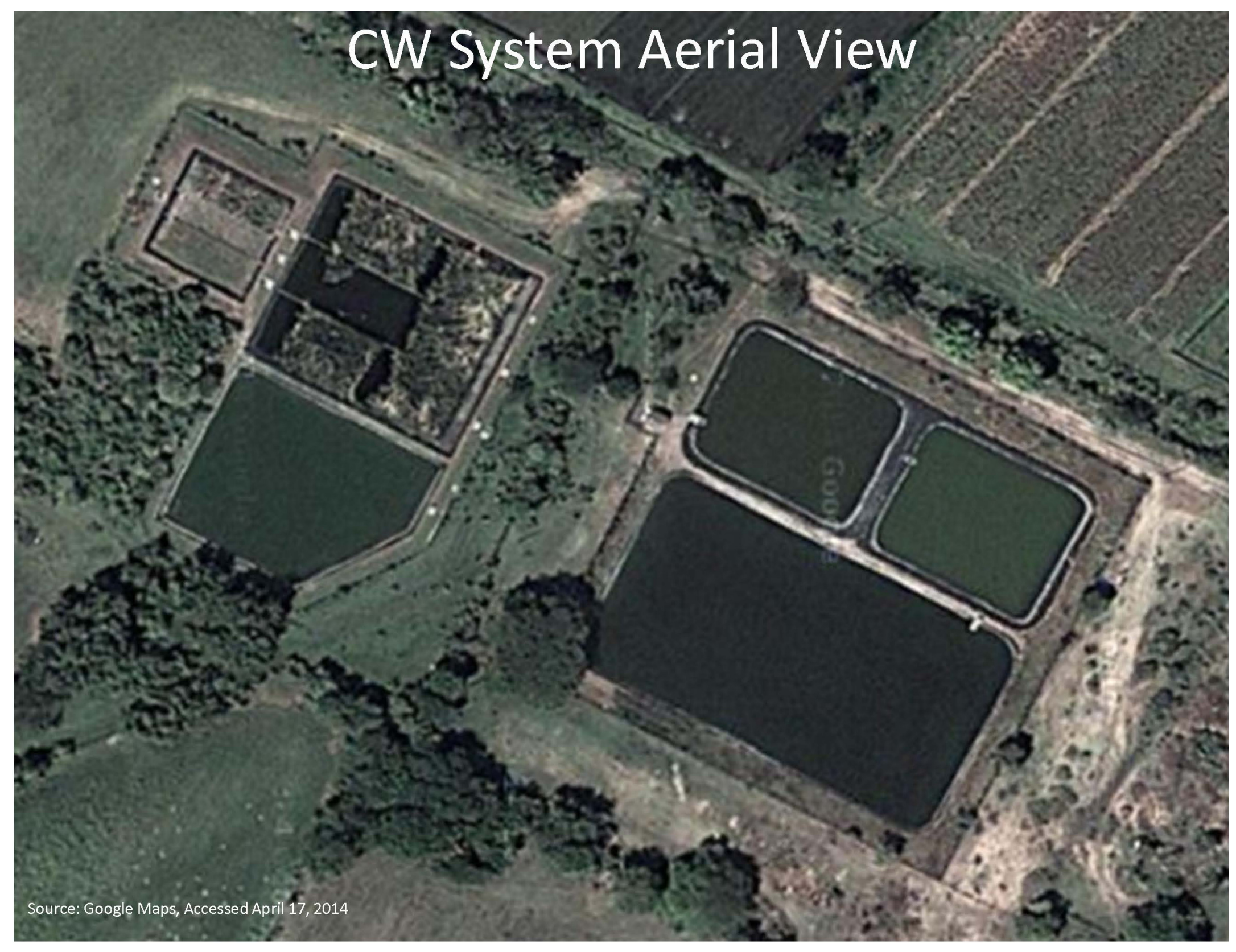




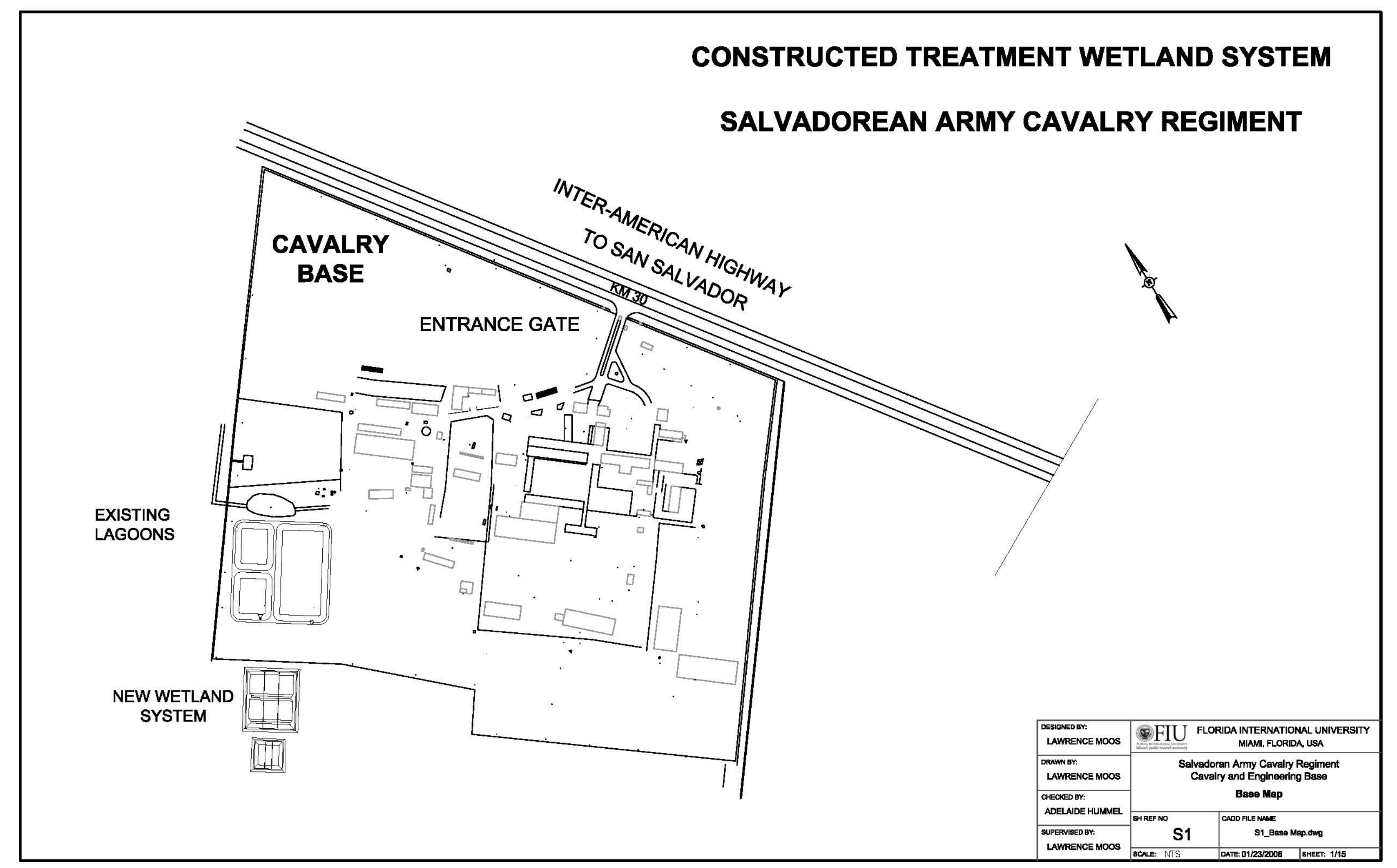




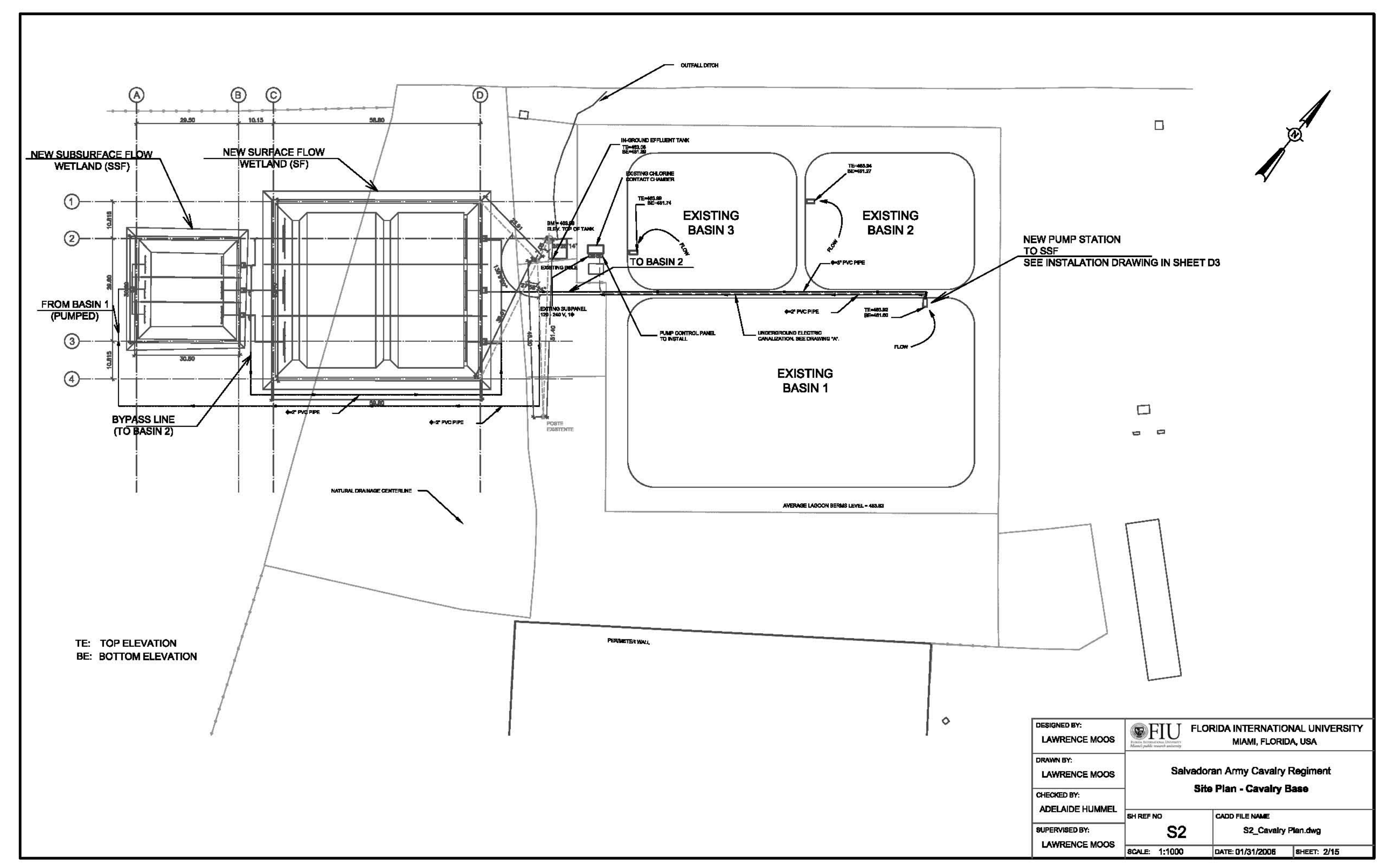




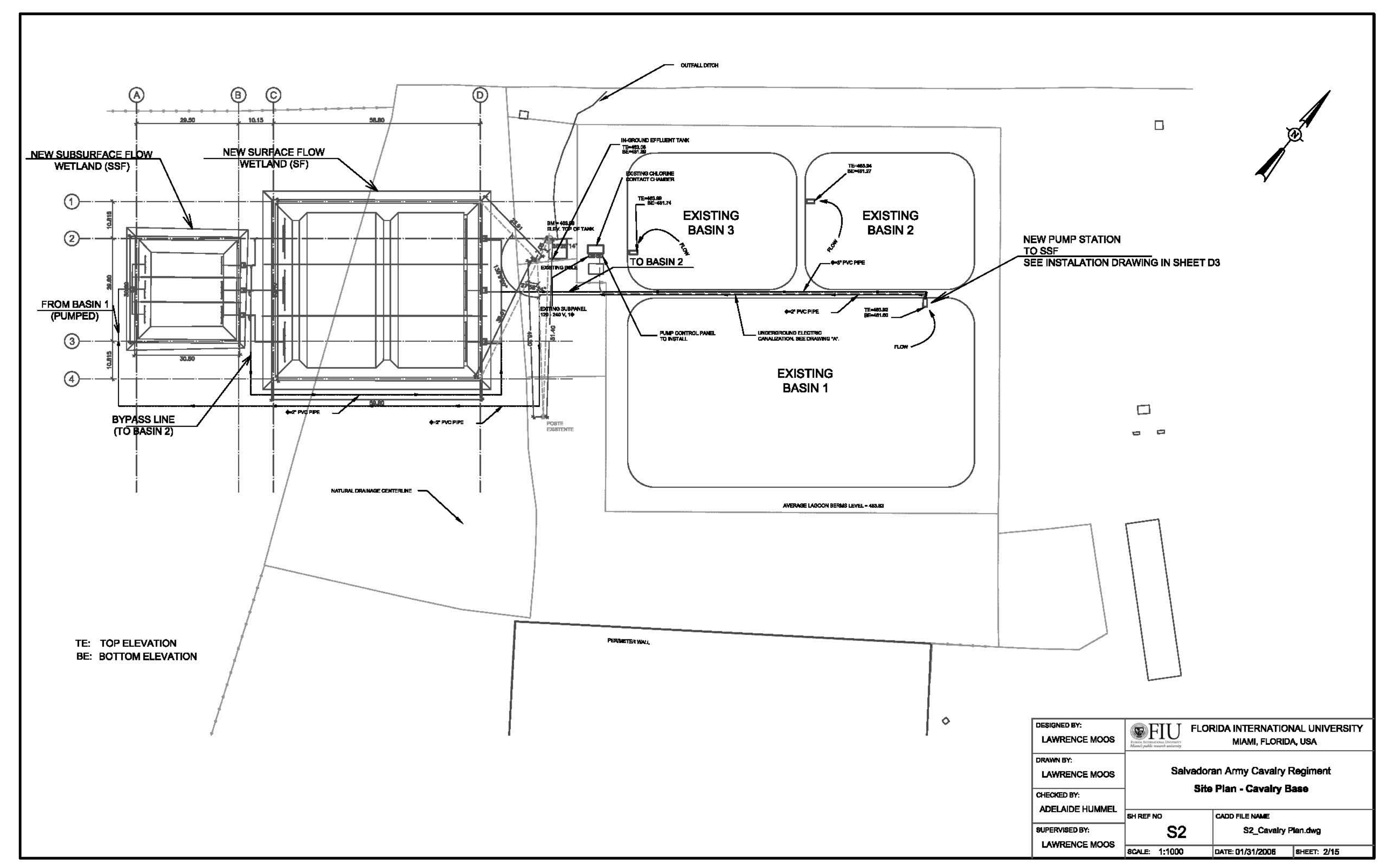




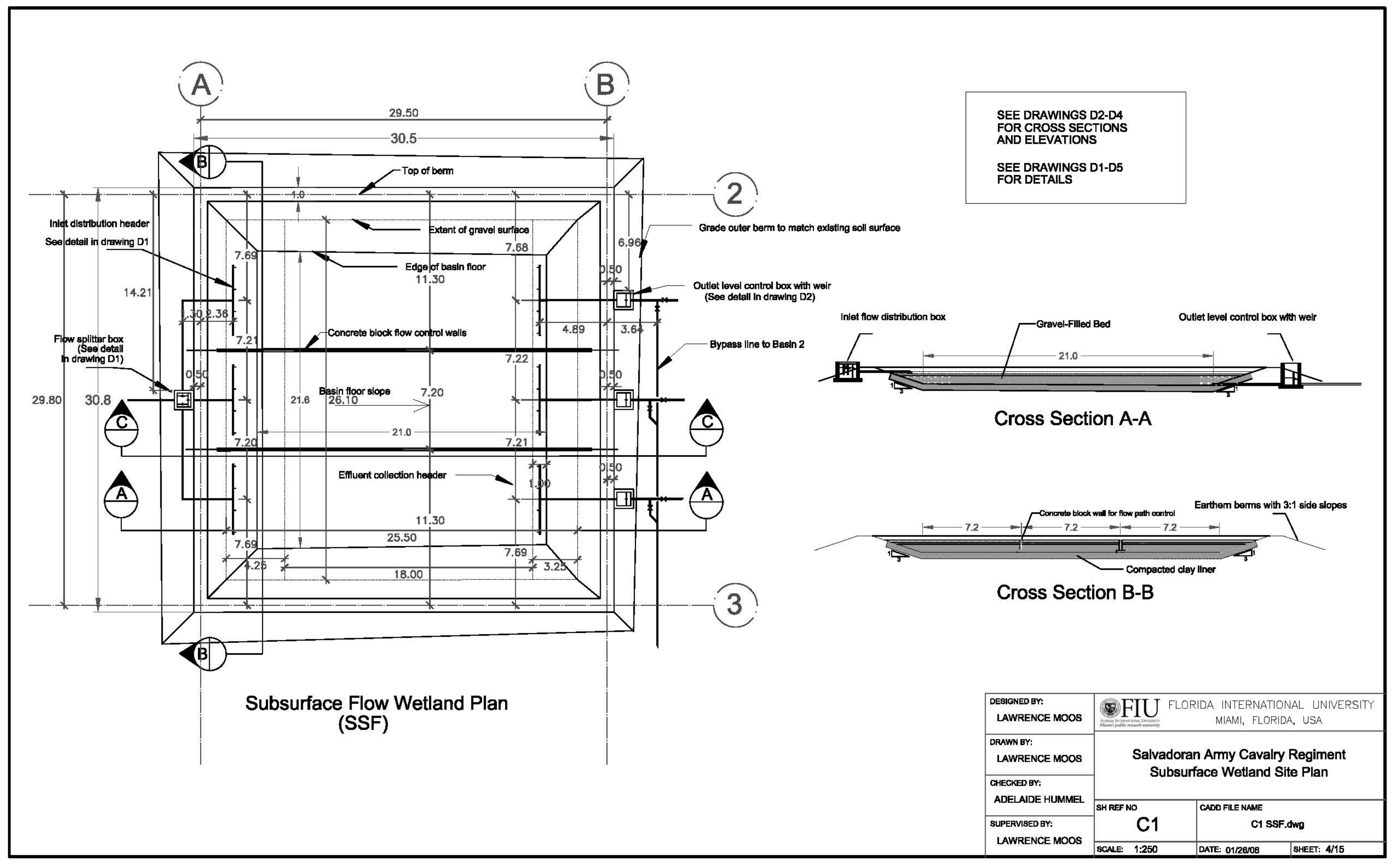




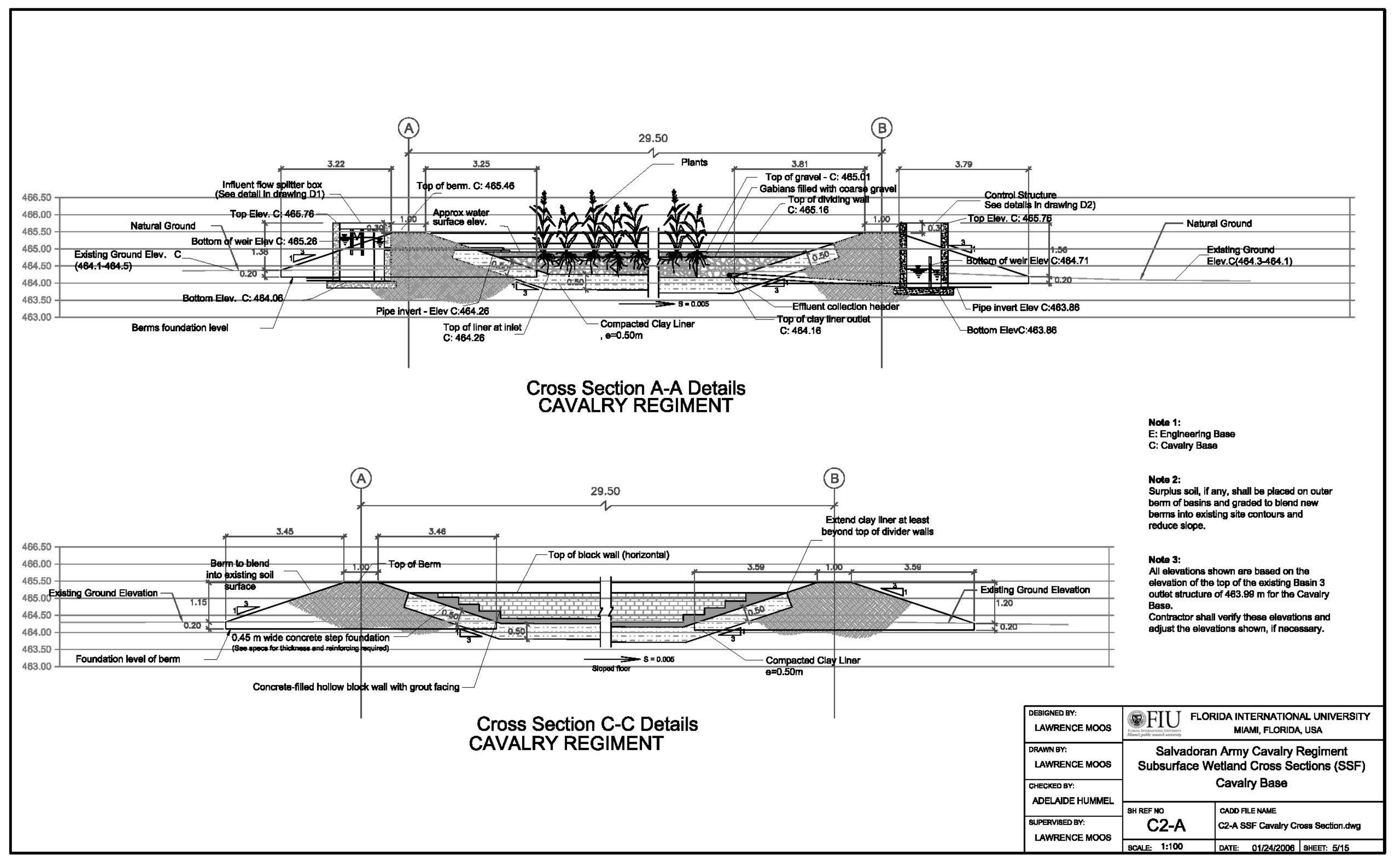




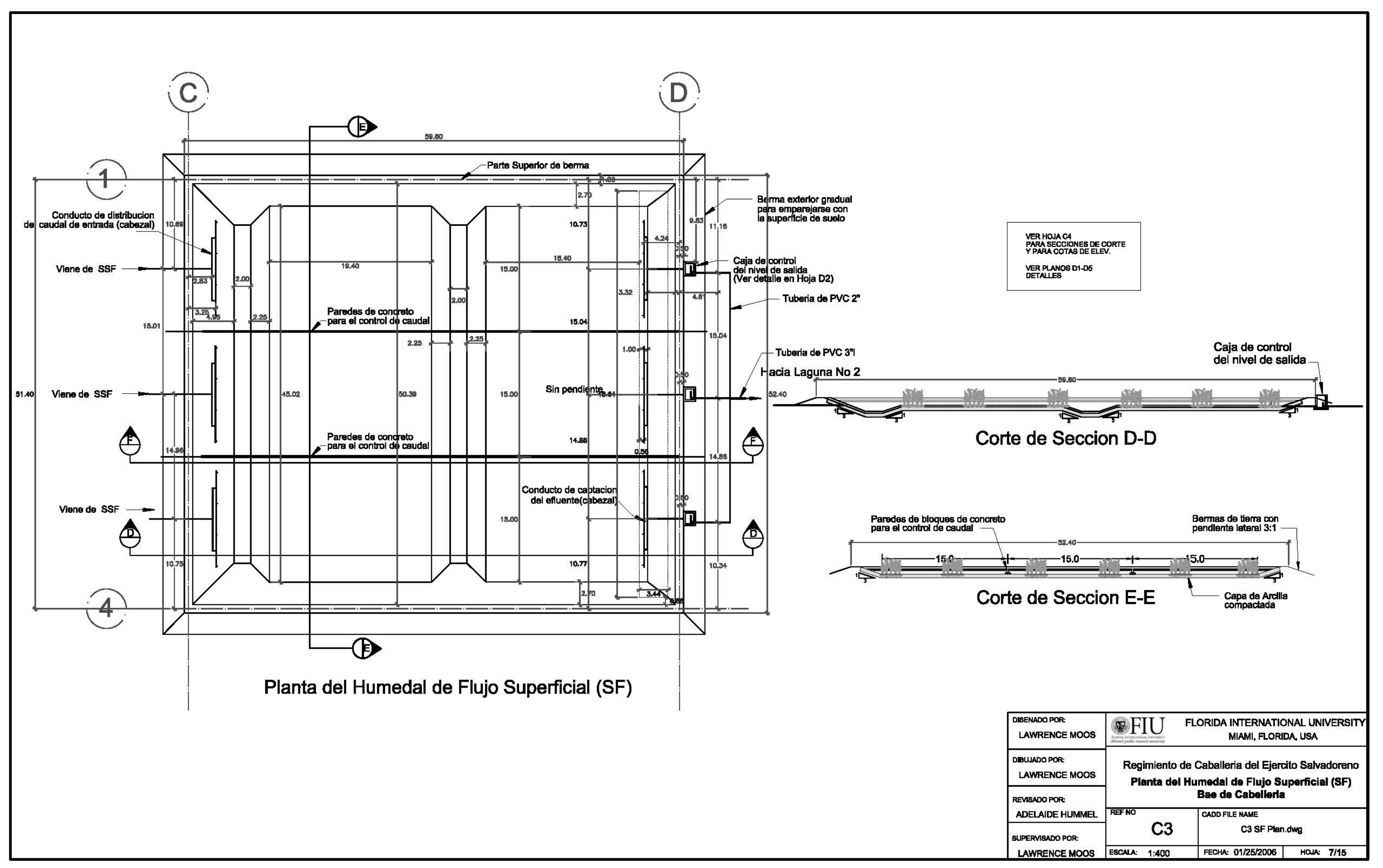




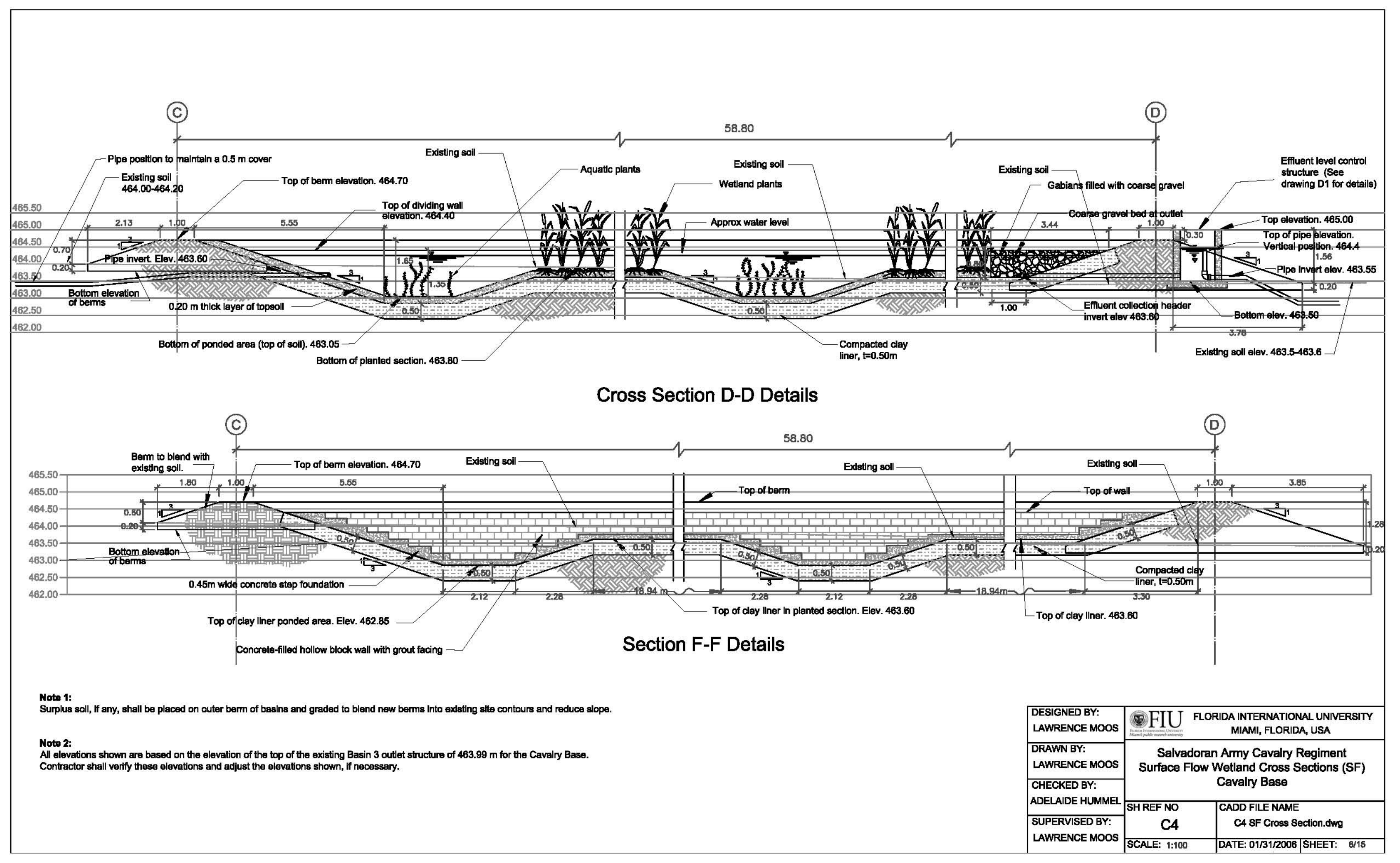




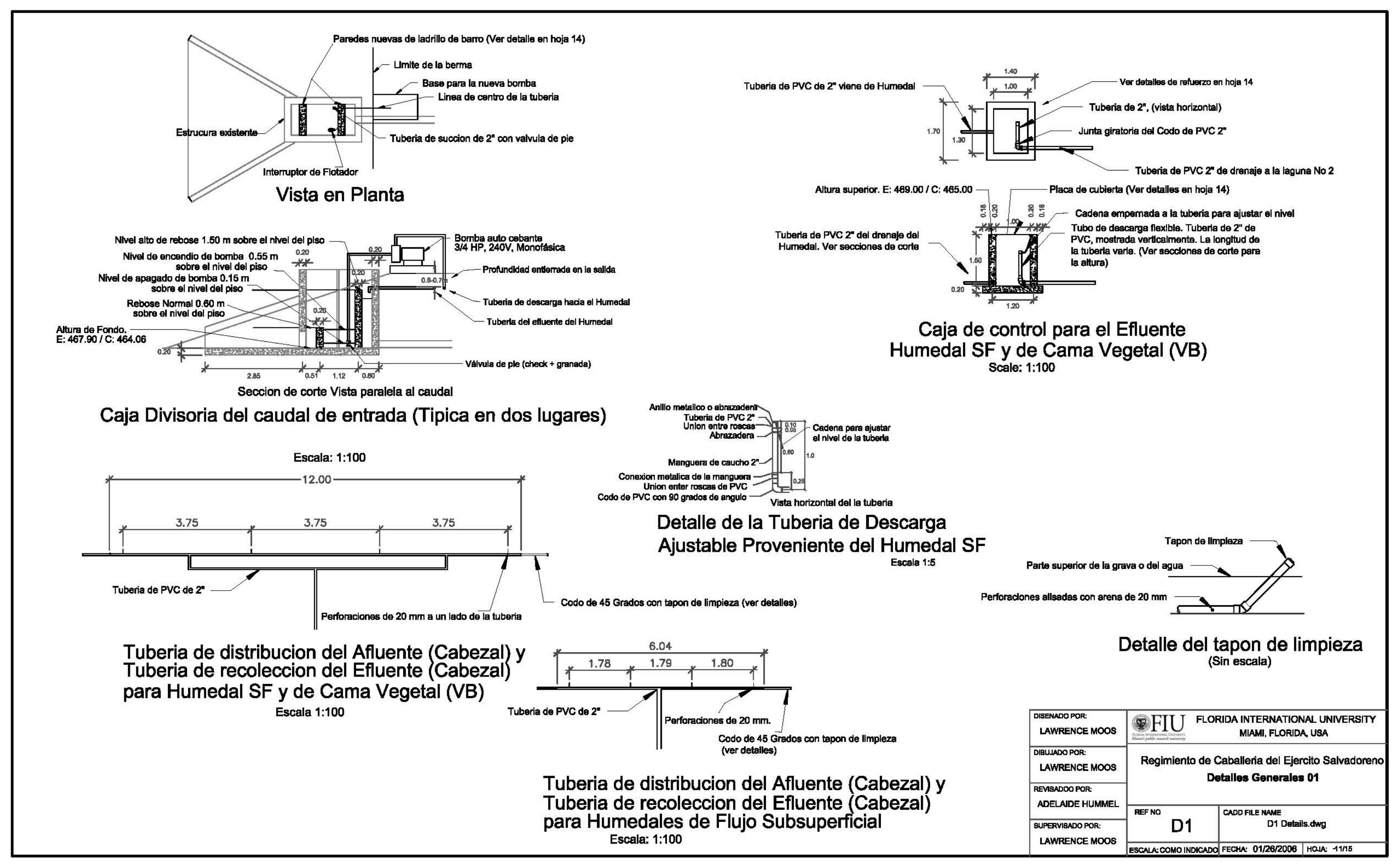




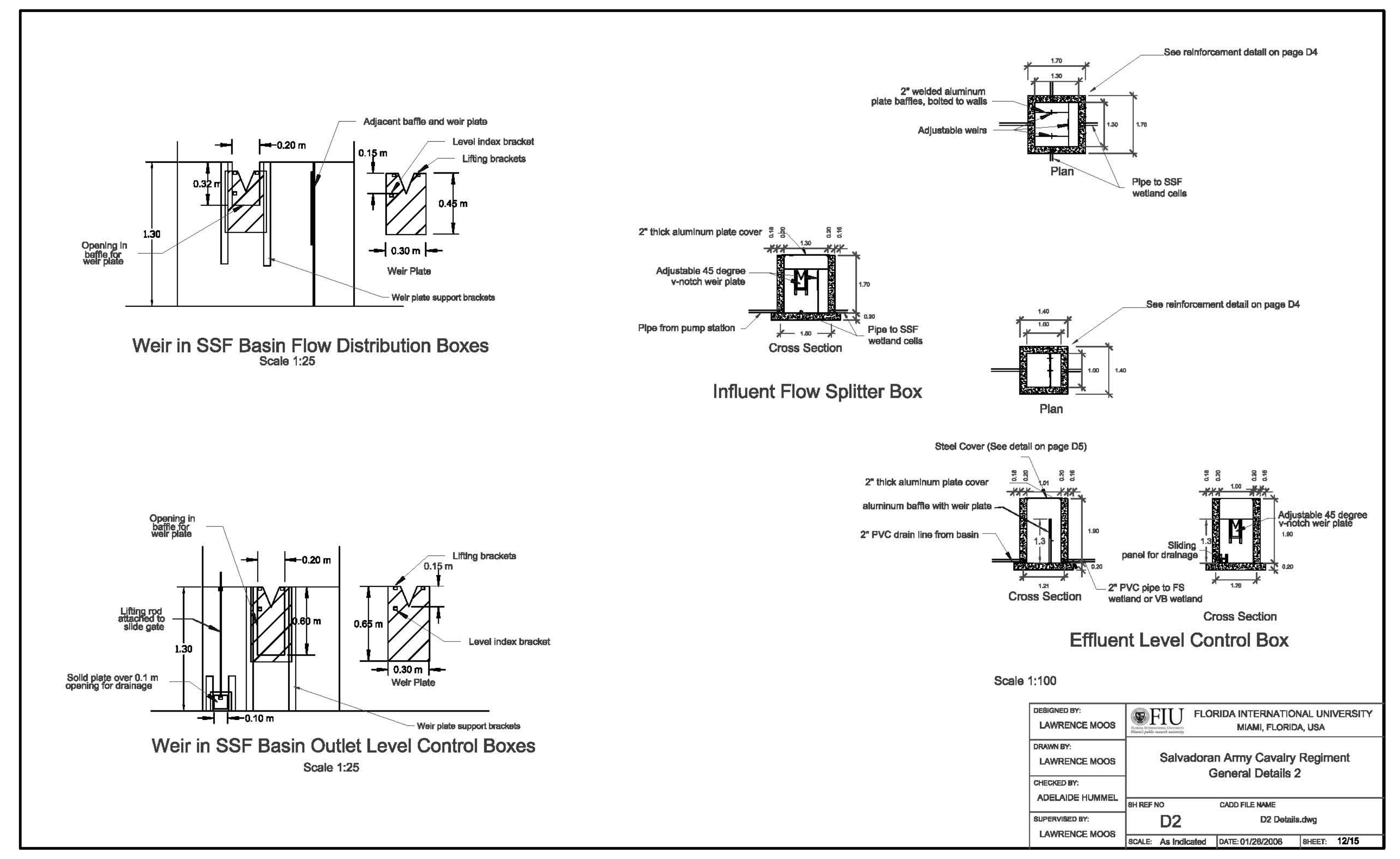




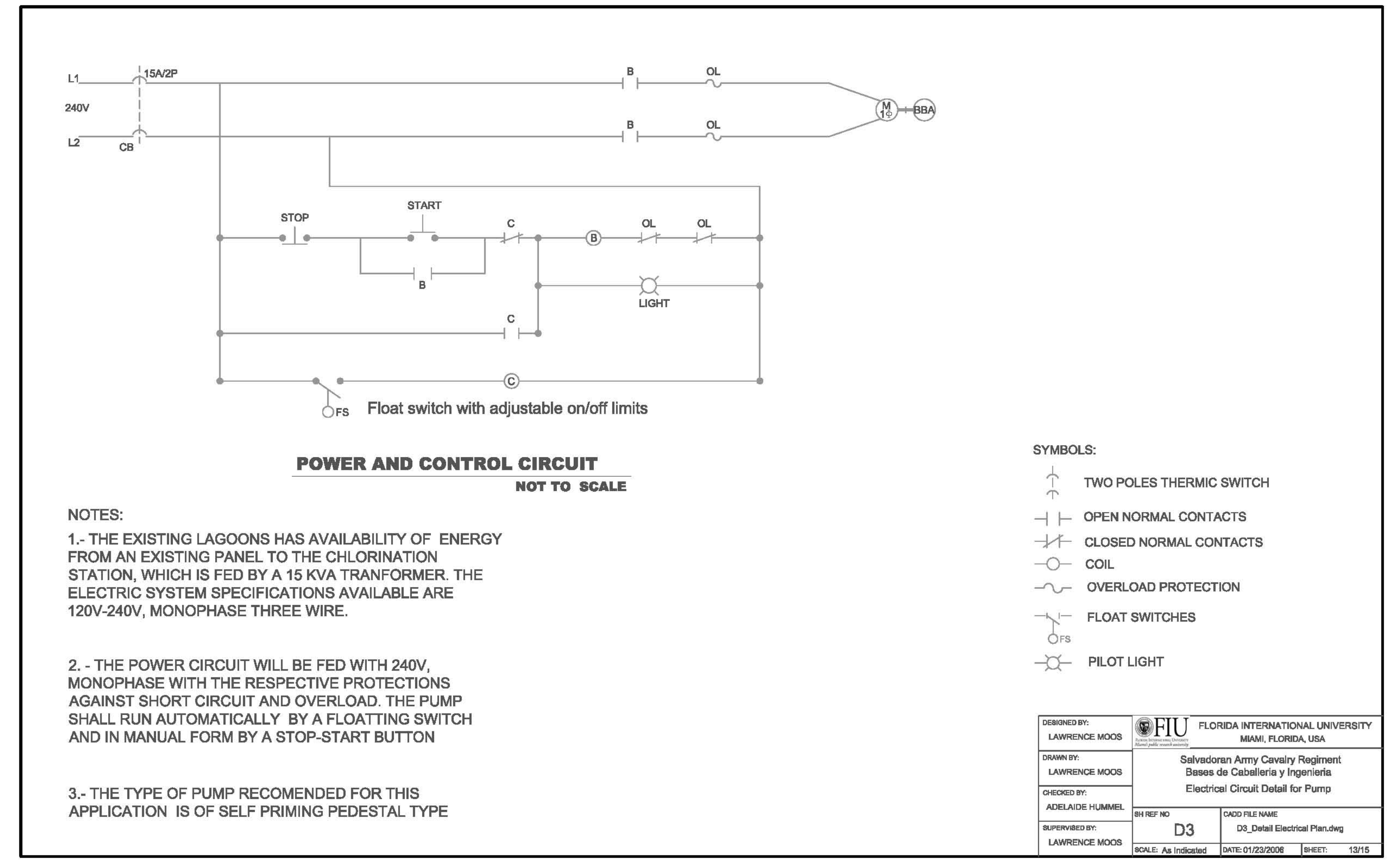




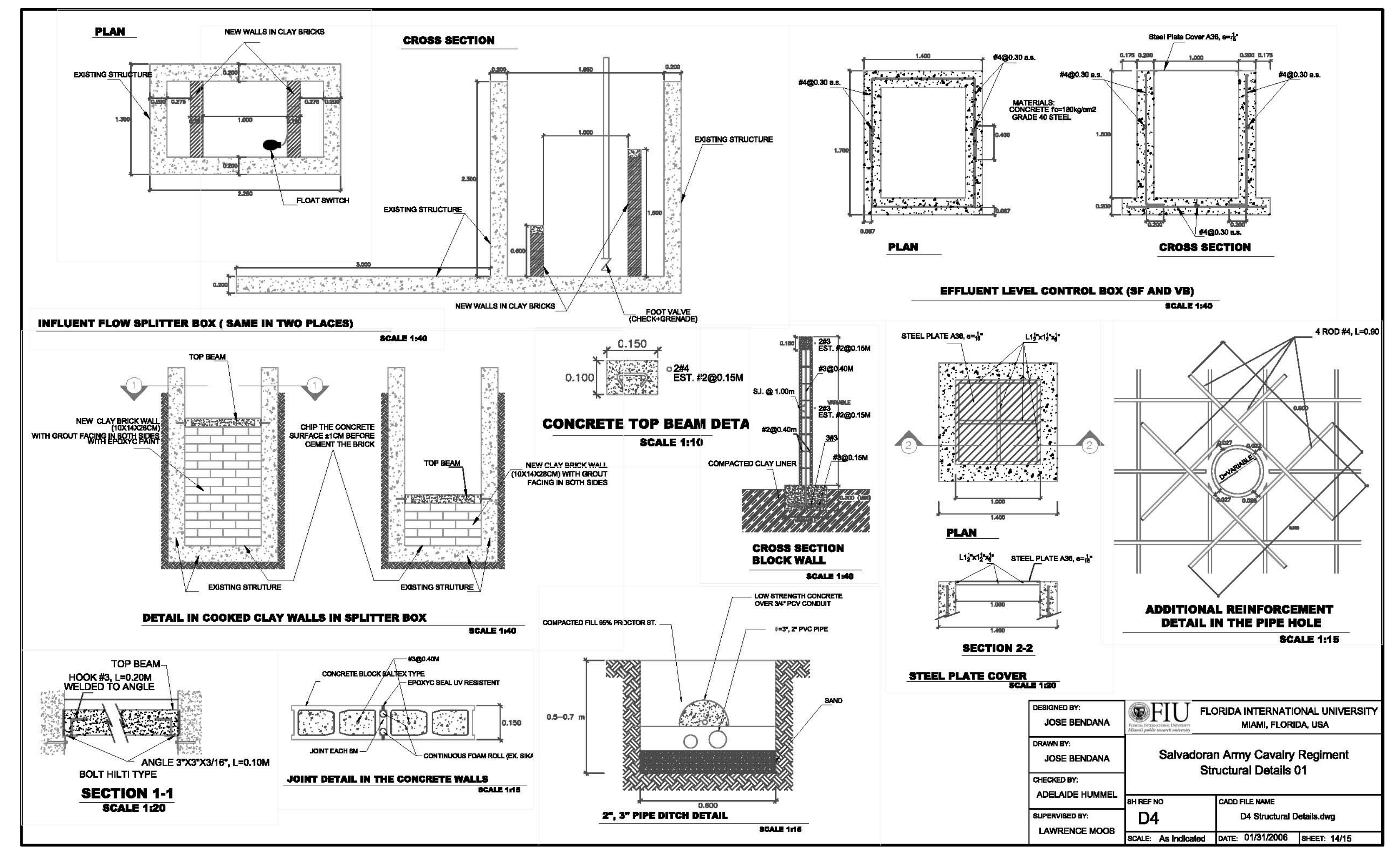




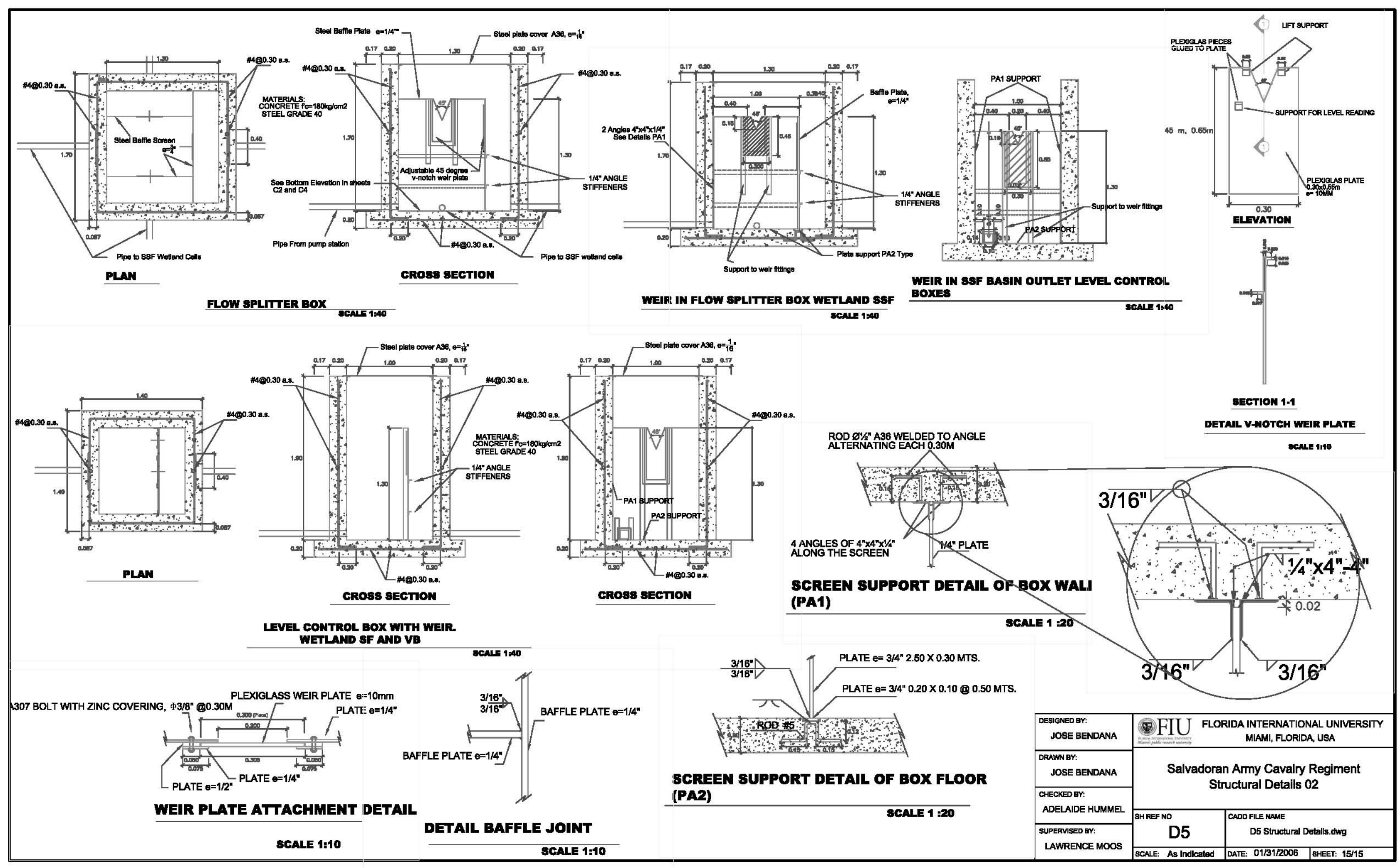




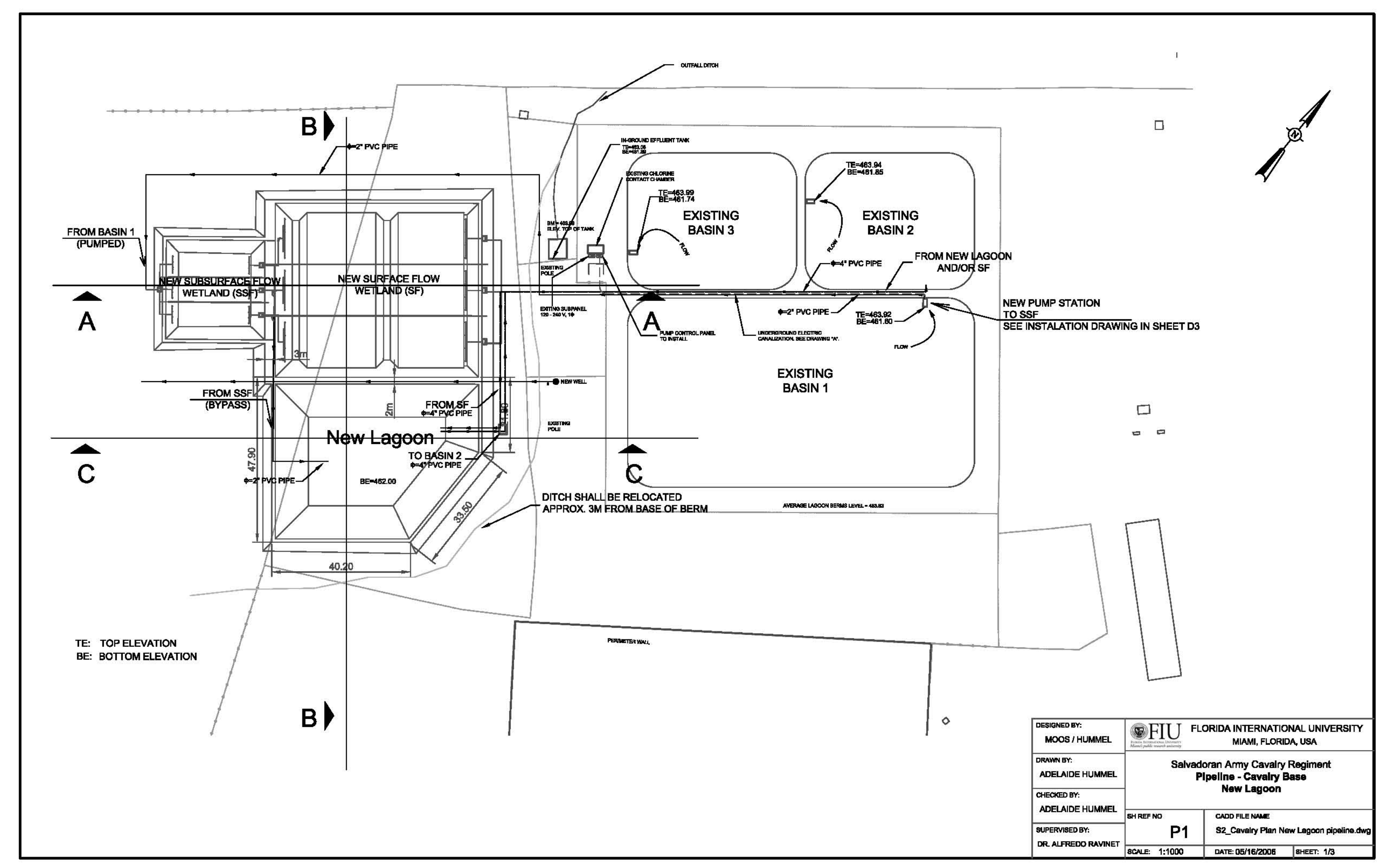




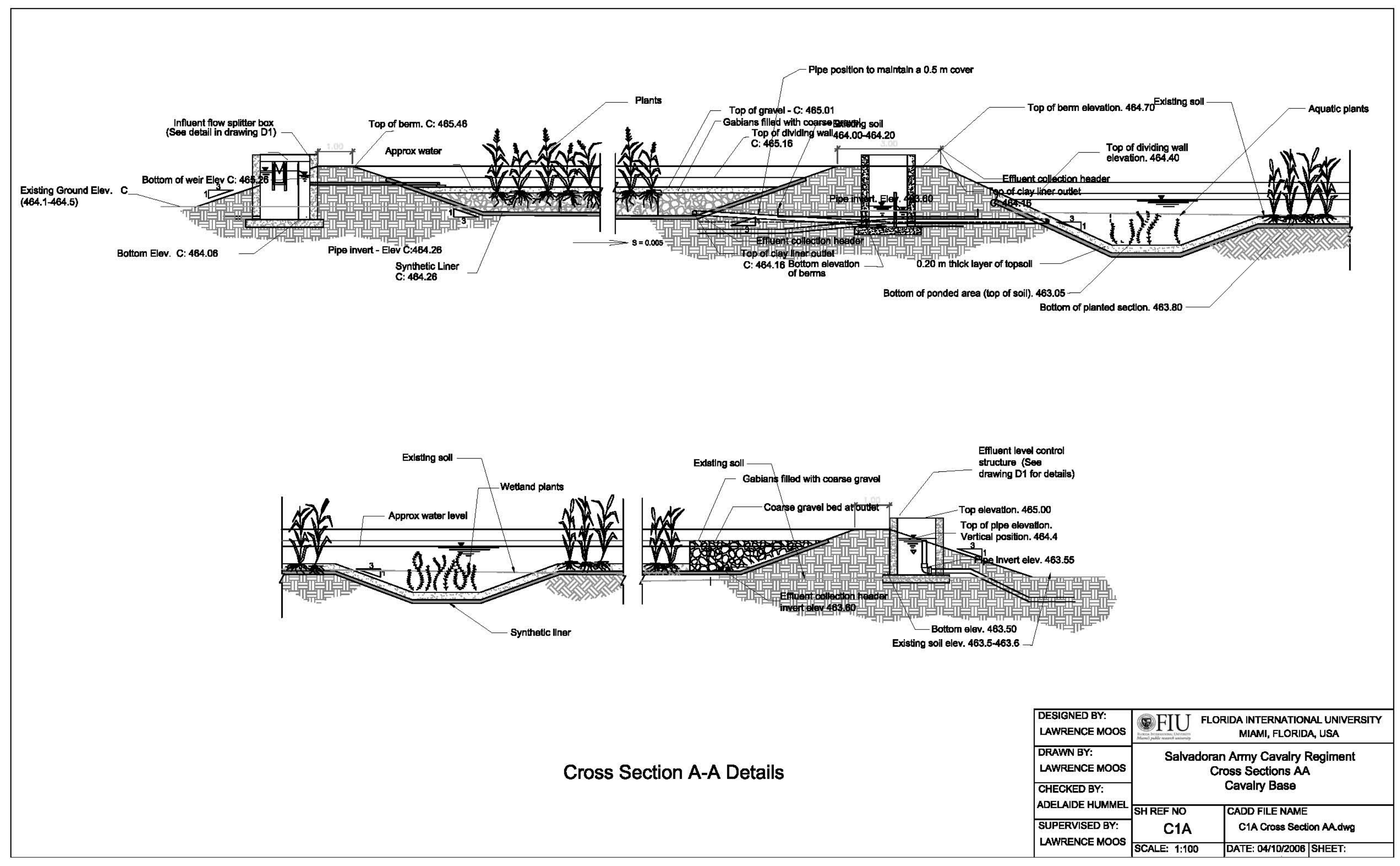




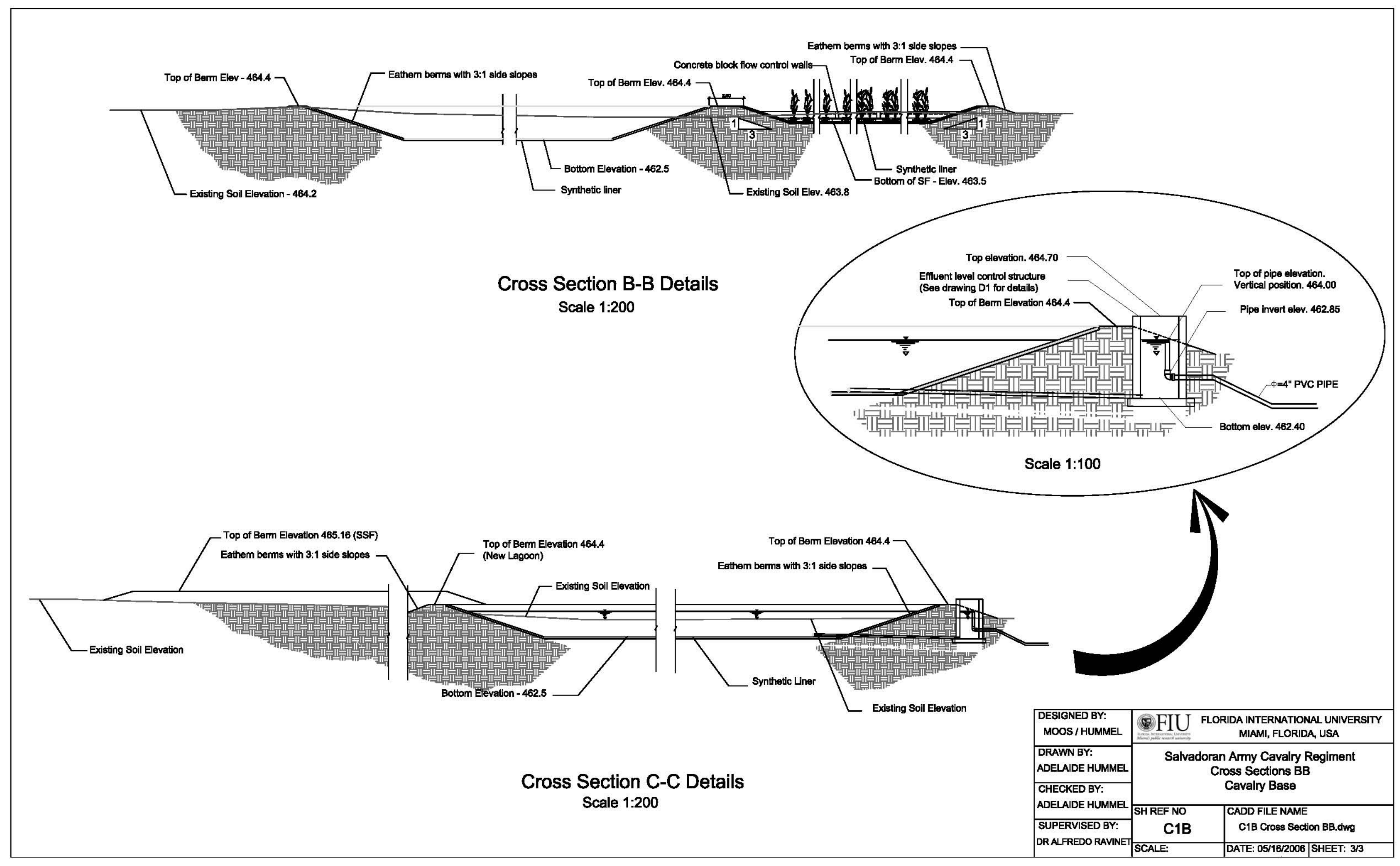




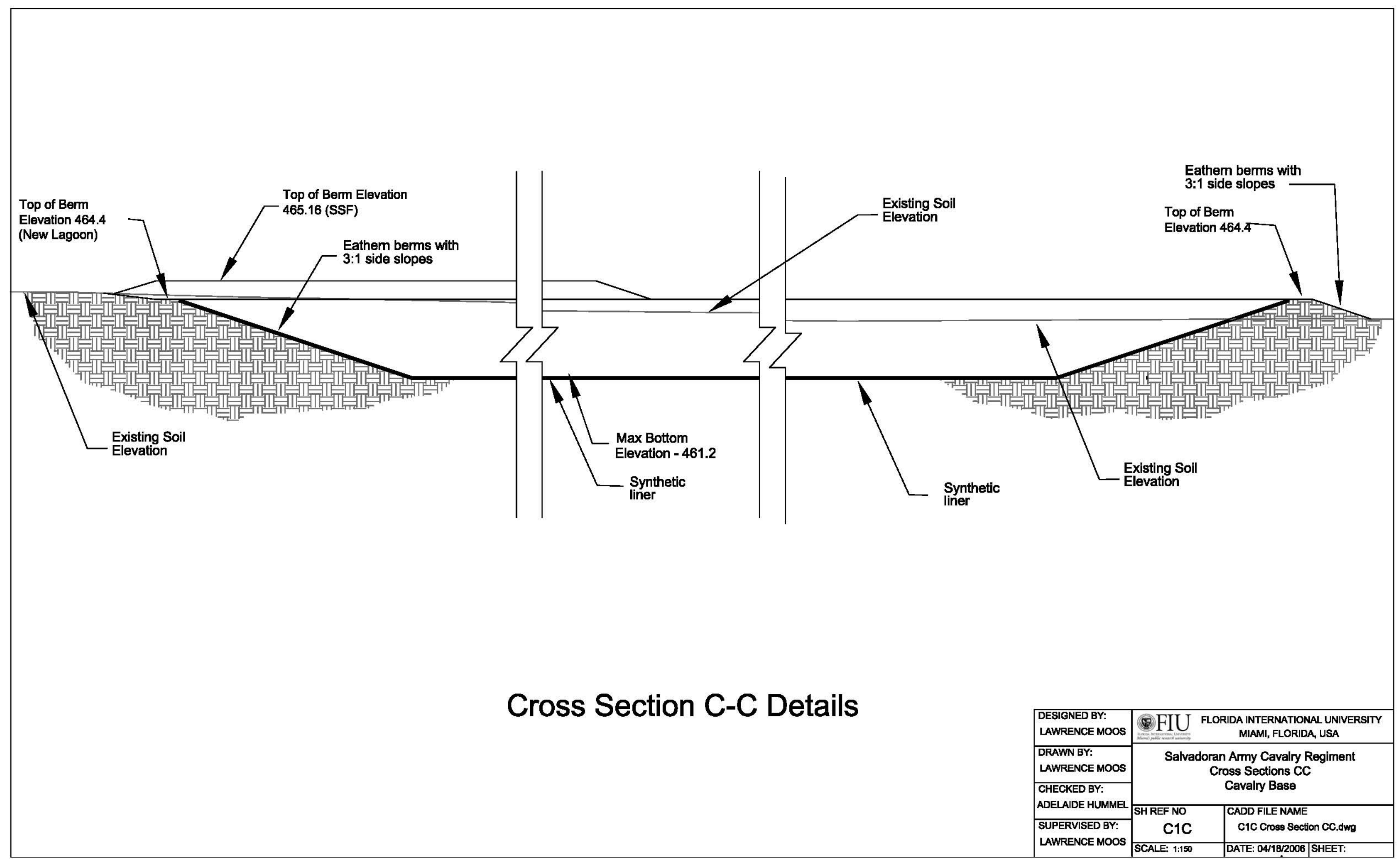


APPENDIX B

DATA SET 
Table B-1 SSF Influent Data (SSF 1)

\begin{tabular}{|c|c|c|c|c|c|c|c|c|c|c|c|}
\hline $\begin{array}{l}\text { Sampling } \\
\text { Date }\end{array}$ & $\begin{array}{l}\text { Fecal Coliform } \\
\text { (NMP/100 mL) }\end{array}$ & $\begin{array}{c}\text { Phosphorus } \\
(\mathrm{mg} / \mathrm{L})\end{array}$ & $\begin{array}{c}\text { Nitrates } \\
(\mathrm{mg} / \mathrm{L})\end{array}$ & $\begin{array}{c}\text { Nitrites } \\
(\mathrm{mg} / \mathrm{L})\end{array}$ & $\begin{array}{c}\text { TDS } \\
(\mathrm{mg} / \mathrm{L})\end{array}$ & $\begin{array}{c}\text { BOD } \\
(\mathrm{mg} / \mathrm{L})\end{array}$ & $\begin{array}{c}\text { COD } \\
(\mathrm{mg} / \mathrm{L})\end{array}$ & $\begin{array}{c}\text { Oil \& Grease } \\
(\mathrm{mg} / \mathrm{L})\end{array}$ & $\begin{array}{c}\text { TSS } \\
(\mathrm{mg} / \mathrm{L})\end{array}$ & $\begin{array}{c}\text { Total Nitrogen } \\
(\mathrm{mg} / \mathrm{L})\end{array}$ & $\begin{array}{c}\text { Ammonium } \\
(\mathrm{mg} / \mathrm{L})\end{array}$ \\
\hline $11 / 29 / 06$ & 300,000 & 2.40 & 2.23 & 0.0 & 320.0 & 72.43 & 223.21 & 4.0 & 114 & 24.46 & 1.08 \\
\hline $12 / 19 / 06$ & 50,000 & 0.91 & 3.82 & 0.0 & 420.0 & 152.27 & 304.88 & 9.4 & 205 & 24.83 & 5.16 \\
\hline 01/08/07 & 24,000 & 1.30 & 11.31 & 0.0 & 450.0 & 80.04 & 142.28 & 0.5 & 140 & 16.56 & 0.16 \\
\hline 01/17/07 & - & 1.42 & 3.08 & 0.0 & 489.5 & 65.43 & 267.58 & 7.0 & 176 & 5.52 & 0.17 \\
\hline $01 / 24 / 07$ & 90,000 & 1.17 & 3.01 & 0.0 & 522.0 & 80.55 & 222.22 & 0.6 & 136 & 2.76 & 0.19 \\
\hline 01/30/07 & 30,000 & 1.35 & 2.94 & 0.0 & 524.5 & 83.18 & 252.02 & 1.4 & 168 & 11.04 & 0.67 \\
\hline $02 / 07 / 07$ & 50,000 & 1.36 & 3.46 & 0.0 & 645.5 & 67.90 & 324.80 & 3.5 & 160 & 17.94 & 2.71 \\
\hline 03/13/07 & 80,000 & 3.31 & 9.12 & 0.0 & 808.5 & 72.48 & 922.11 & 7.1 & 335 & 41.59 & - \\
\hline $04 / 24 / 07$ & $1,600,000$ & 0.83 & 5.59 & 0.0 & 229.5 & 48.20 & 397.29 & 20.2 & 630 & 25.74 & 6.56 \\
\hline 05/09/07 & 300,000 & 1.15 & 5.66 & 0.0 & 484.5 & 32.81 & 184.87 & 3.4 & 152 & 20.13 & 2.25 \\
\hline $06 / 27 / 07$ & 50,000 & 3.58 & 15.43 & 0.0 & 617.5 & 63.27 & 216.00 & 16.0 & 100 & 21.01 & 7.40 \\
\hline 07/04/07 & 2,400 & 3.41 & 3.48 & 0.8 & 606.0 & 43.59 & 51.59 & 0.8 & 30 & 17.51 & 14.67 \\
\hline 07/11/07 & 50,000 & 2.41 & 4.57 & 0.0 & 606.5 & 43.16 & 96.00 & 9.4 & 25 & 17.14 & 9.51 \\
\hline $07 / 18 / 07$ & 140,000 & 0.48 & 1.35 & 0.0 & 474.5 & 79.99 & 316.00 & 5.4 & 72 & 17.67 & 12.69 \\
\hline $07 / 25 / 07$ & 30,000 & 0.53 & 2.59 & 0.0 & 510.5 & 42.64 & 180.00 & 1.2 & 102 & 25.16 & 21.85 \\
\hline $08 / 15 / 07$ & 90,000 & 0.57 & 8.47 & 0.0 & 563.0 & 63.44 & 214.29 & 4.1 & 48 & 26.84 & 15.80 \\
\hline 09/19/07 & 50,000 & 4.69 & 0.57 & - & - & 42.92 & - & 5.6 & - & - & 15.84 \\
\hline Average & 183,525 & 1.82 & 5.10 & 0.1 & 517.0 & 66.72 & 269.70 & 5.9 & 162 & 19.74 & 7.29 \\
\hline $\begin{array}{l}\text { Standard } \\
\text { Deviation }\end{array}$ & 388,002 & 1.26 & 3.90 & 0.2 & 133.9 & 27.34 & 194.64 & 5.5 & 146 & 9.09 & 6.99 \\
\hline
\end{tabular}




\begin{tabular}{|c|c|c|c|c|c|c|c|c|c|c|c|}
\hline \multicolumn{12}{|c|}{ Table B-2 SSF Effluent (SSF 1-2 Phragmites) } \\
\hline $\begin{array}{l}\text { Sampling } \\
\text { Date }\end{array}$ & $\begin{array}{l}\text { Fecal Coliform } \\
\text { (NMP/100 mL) }\end{array}$ & $\begin{array}{c}\text { Phosphorus } \\
(\mathrm{mg} / \mathrm{L})\end{array}$ & $\begin{array}{l}\text { Nitrates } \\
(\mathrm{mg} / \mathrm{L})\end{array}$ & $\begin{array}{c}\text { Nitrites } \\
(\mathrm{mg} / \mathrm{L})\end{array}$ & $\begin{array}{c}\text { TDS } \\
(\mathrm{mg} / \mathrm{L})\end{array}$ & $\begin{array}{c}\text { BOD } \\
(\mathrm{mg} / \mathrm{L})\end{array}$ & $\begin{array}{c}\text { COD } \\
(\mathrm{mg} / \mathrm{L})\end{array}$ & $\begin{array}{c}\text { Oil \& Grease } \\
(\mathrm{mg} / \mathrm{L})\end{array}$ & $\begin{array}{c}\text { TSS } \\
(\mathrm{mg} / \mathrm{L})\end{array}$ & $\begin{array}{c}\text { Total Nitrogen } \\
(\mathrm{mg} / \mathrm{L})\end{array}$ & $\underset{(\mathrm{mg} / \mathrm{L})}{\operatorname{Ammonium}}$ \\
\hline $11 / 29 / 06$ & 50,000 & 2.22 & 2.09 & 0.00 & 330.0 & 44.76 & 128.97 & 4.0 & 30 & 16.30 & 2.85 \\
\hline $12 / 19 / 06$ & 16,000 & 0.42 & 2.76 & 0.00 & 430.0 & 92.33 & 172.76 & 7.4 & 80 & 15.18 & 4.60 \\
\hline 01/08/07 & 3,000 & 4.02 & 10.71 & 0.00 & 535.0 & 66.31 & 130.08 & 0.4 & 34 & 11.04 & 5.56 \\
\hline 01/17/07 & - & 2.37 & 2.16 & 0.00 & 536.0 & 46.76 & 175.78 & 3.6 & 88 & 13.80 & 1.75 \\
\hline $01 / 24 / 07$ & 16,000 & 2.09 & 10.28 & 0.00 & 528.0 & 62.16 & 106.48 & 0.6 & 4 & 11.04 & 3.24 \\
\hline 01/30/07 & 110 & 2.46 & 6.11 & 0.00 & 524.5 & 52.77 & 206.65 & 0.6 & 4 & 5.52 & 6.42 \\
\hline 02/07/07 & 130 & 2.57 & 3.65 & 0.00 & 598.0 & 56.36 & 114.17 & 2.5 & 6 & 5.52 & 11.51 \\
\hline 03/13/07 & 17,000 & 3.54 & 8.00 & 0.00 & 753.5 & 61.90 & 139.34 & 6.6 & 25 & 14.51 & - \\
\hline $04 / 24 / 07$ & 16,000 & 3.36 & 1.20 & 0.00 & 686.5 & 63.83 & 131.78 & 5.9 & 16 & 19.70 & 13.69 \\
\hline 05/09/07 & 240 & 3.59 & 19.56 & 0.00 & 518.5 & 78.10 & 184.87 & 9.5 & 4 & 23.76 & 18.69 \\
\hline 06/27/07 & 30,000 & 2.81 & 2.71 & 0.00 & 516.0 & 32.60 & 80.00 & 4.3 & 0 & 13.18 & 8.66 \\
\hline 07/04/07 & 240 & 2.97 & 19.68 & 0.02 & 554.5 & 41.18 & 47.62 & 3.8 & 0 & 14.20 & 12.52 \\
\hline 07/11/07 & 9,000 & 3.63 & 3.19 & 0.03 & 578.5 & 28.45 & 56.00 & 0.3 & 6 & 13.94 & 3.42 \\
\hline 07/18/07 & 2,400 & 2.73 & 0.66 & 0.00 & 411.0 & 64.32 & 112.00 & 3.1 & 16 & 8.07 & 5.35 \\
\hline $07 / 25 / 07$ & 9,000 & 2.02 & 0.97 & 0.34 & 439.0 & 16.93 & 20.00 & 8.5 & 12 & 12.02 & 11.16 \\
\hline 08/15/07 & 3,000 & 4.73 & 0.25 & 0.00 & 459.5 & 35.98 & 60.15 & 1.4 & 0 & 10.56 & 10.39 \\
\hline 09/19/07 & 11,000 & 4.28 & 0.78 & - & - & 13.67 & - & 1.0 & - & - & 7.89 \\
\hline Average & 11,445 & 2.93 & 5.57 & 0.02 & 524.9 & 50.49 & 116.67 & 3.7 & 20 & 13.02 & 7.98 \\
\hline $\begin{array}{l}\text { Standard } \\
\text { Deviation }\end{array}$ & 13,337 & 1.02 & 6.20 & 0.08 & 103.1 & 21.02 & $\mathbf{5 3 . 5 0}$ & 3.0 & 27 & 4.71 & 4.70 \\
\hline
\end{tabular}




\begin{tabular}{|c|c|c|c|c|c|c|c|c|c|c|c|}
\hline \multicolumn{12}{|c|}{ Table B-3 SSF Effluent (SSF 1-3 Thalia) } \\
\hline $\begin{array}{l}\text { Sampling } \\
\text { Date }\end{array}$ & $\begin{array}{l}\text { Fecal Coliform } \\
\text { (NMP/100 mL) }\end{array}$ & $\begin{array}{c}\text { Phosphorus } \\
(\mathrm{mg} / \mathrm{L})\end{array}$ & $\begin{array}{l}\text { Nitrates } \\
(\mathrm{mg} / \mathrm{L})\end{array}$ & $\begin{array}{c}\text { Nitrites } \\
(\mathrm{mg} / \mathrm{L})\end{array}$ & $\begin{array}{c}\text { TDS } \\
(\mathrm{mg} / \mathrm{L})\end{array}$ & $\begin{array}{c}\text { BOD } \\
(\mathrm{mg} / \mathrm{L})\end{array}$ & $\begin{array}{c}\text { COD } \\
(\mathrm{mg} / \mathrm{L})\end{array}$ & $\begin{array}{c}\text { Oil \& Grease } \\
(\mathrm{mg} / \mathrm{L})\end{array}$ & $\begin{array}{c}\text { TSS } \\
(\mathrm{mg} / \mathrm{L})\end{array}$ & $\begin{array}{c}\text { Total Nitrogen } \\
(\mathrm{mg} / \mathrm{L})\end{array}$ & $\underset{(\mathrm{mg} / \mathrm{L})}{\operatorname{Ammonium}}$ \\
\hline $11 / 29 / 06$ & 50,000 & 2.16 & 2.55 & 0 & 330 & 41.29 & 158.73 & 3.4 & 16 & 13.59 & 3.21 \\
\hline $12 / 19 / 06$ & 50,000 & 0.29 & 1.89 & 0 & 460 & 167.4 & 238.82 & 8.6 & 95 & 16.56 & 8.14 \\
\hline $01 / 08 / 07$ & 24,000 & 3.92 & 8.29 & 0 & 522 & 48.24 & 113.82 & 0.9 & 42 & 13.8 & 7.06 \\
\hline $01 / 17 / 07$ & - & 2.08 & 2.06 & 0 & 512 & 39.16 & 171.88 & 1 & 104 & 8.28 & 1.22 \\
\hline $01 / 24 / 07$ & 16,000 & 2.00 & 4.21 & 0 & 528.5 & 66.78 & 175.93 & 0.6 & 20 & 10.35 & 4.47 \\
\hline 01/30/07 & 70 & 2.23 & 4.02 & 0 & 514.5 & 65.56 & 241.94 & 0.6 & 4 & 3.45 & 7.59 \\
\hline 02/07/07 & 17,000 & 2.57 & 3.63 & 0 & 645.5 & 93.65 & 127.95 & 0.9 & 4 & 12.42 & 13.86 \\
\hline 03/13/07 & 22,000 & 3.53 & 11.25 & 0 & 746.5 & 61.03 & 196.72 & 2.3 & 30 & 16.29 & - \\
\hline $04 / 24 / 07$ & 16,000 & 2.62 & 0.27 & 0 & 615.5 & 66.03 & 147.29 & 5.9 & 18 & 19.87 & 13.69 \\
\hline 05/09/07 & 17,000 & 2.97 & 14.62 & 0 & 520 & 32.06 & 193.28 & 7.9 & 0 & 18.24 & 14.16 \\
\hline 06/27/07 & 13,000 & 3.56 & 5.47 & 0 & 507.5 & 31.02 & 144 & 2.2 & 0 & 12.66 & 9.13 \\
\hline 07/04/07 & 5,000 & 3.47 & 19.68 & 4 & 551.5 & 36.05 & 45.63 & 1 & 0 & 15.63 & 11.88 \\
\hline 07/11/07 & 30,000 & 3.71 & 5.54 & 0.03 & 547.5 & 25.74 & 56 & 1.3 & 4 & 14.11 & 9.22 \\
\hline 07/18/07 & 30,000 & 1.60 & 2.67 & 0 & 422.5 & 55.12 & 112 & 7.9 & 18 & 8.65 & 7.37 \\
\hline $07 / 25 / 07$ & 80,000 & 0.47 & 1.57 & 0.61 & 478 & 27.06 & 92 & 8.6 & 42 & 11.49 & 10.14 \\
\hline 08/15/07 & 16,000 & 4.06 & 0.59 & 0 & 471 & 30.23 & 48.87 & 1.2 & 0 & 11.01 & 8.73 \\
\hline 09/19/07 & 50,000 & 4.25 & 0.72 & - & - & 21.33 & - & 0.2 & - & - & 7.34 \\
\hline Average & 27,254 & 2.68 & 5.24 & 0.29 & 523.3 & 53.40 & 141.55 & 3.2 & 25 & 12.90 & 8.58 \\
\hline $\begin{array}{l}\text { Standard } \\
\text { Deviation }\end{array}$ & 20,680 & 1.18 & 5.38 & 1.00 & 93.6 & 35.20 & 61.69 & 3.2 & 32 & 4.11 & 3.70 \\
\hline
\end{tabular}




\section{Table B-4 SSF Effluent (SSF 1-4 Brachiaria)}

\begin{tabular}{|c|c|c|c|c|c|c|c|c|c|c|c|}
\hline $\begin{array}{l}\text { Sampling } \\
\text { Date }\end{array}$ & $\begin{array}{l}\text { Fecal Coliform } \\
\text { (NMP/100 mL) }\end{array}$ & $\begin{array}{c}\text { Phosphorus } \\
\text { (mg/L) }\end{array}$ & $\begin{array}{c}\text { Nitrates } \\
(\mathrm{mg} / \mathrm{L})\end{array}$ & $\begin{array}{c}\text { Nitrites } \\
\text { (mg/L) }\end{array}$ & $\begin{array}{c}\text { TDS } \\
(\mathrm{mg} / \mathrm{L})\end{array}$ & $\begin{array}{c}\text { BOD } \\
(\mathrm{mg} / \mathrm{L})\end{array}$ & $\begin{array}{c}\text { COD } \\
(\mathrm{mg} / \mathrm{L})\end{array}$ & $\begin{array}{c}\text { Oil \& Grease } \\
(\mathrm{mg} / \mathrm{L})\end{array}$ & $\begin{array}{c}\text { TSS } \\
(\mathrm{mg} / \mathrm{L})\end{array}$ & $\begin{array}{c}\text { Total Nitrogen } \\
(\mathrm{mg} / \mathrm{L})\end{array}$ & $\begin{array}{c}\text { Ammonium } \\
(\mathrm{mg} / \mathrm{L})\end{array}$ \\
\hline $11 / 29 / 06$ & 90,000 & 2.15 & 2.71 & 0 & 320 & 39.23 & 148.81 & 2.6 & 34 & 16.3 & 2.85 \\
\hline $12 / 19 / 06$ & 11,000 & 0.28 & 3.43 & 0 & 420 & 80.67 & 96.54 & 4.8 & 30 & 2.76 & 5.56 \\
\hline 01/08/07 & 800 & 3.87 & 7.19 & 0 & 542.5 & 45.11 & 109.76 & 1.1 & 44 & 8.28 & 5.79 \\
\hline 01/17/07 & - & 2.89 & 2.67 & 0 & 537 & 38.44 & 175.78 & 0.8 & 40 & 11.04 & 4.04 \\
\hline $01 / 24 / 07$ & 50,000 & 1.76 & 6.72 & 0 & 502 & 44.33 & 180.56 & 1.2 & 4 & 8.28 & 4.12 \\
\hline 01/30/07 & 50 & 2.04 & 6.2 & 0 & 489 & 47.95 & 60.48 & 0.5 & 0 & 4.83 & 5.9 \\
\hline $02 / 07 / 07$ & 3,000 & 2.31 & 3.1 & 0 & 590.5 & 36.36 & 102.36 & 1.9 & 16 & 9.66 & 12.4 \\
\hline 03/13/07 & 13,000 & 3.58 & 11.98 & 0 & 697.5 & 42.57 & 163.93 & 2.5 & 40 & 15.21 & - \\
\hline $04 / 24 / 07$ & 30,000 & 2.70 & 3.68 & 0 & 615 & 55.99 & 85.27 & 0.4 & 8 & 17.86 & 12.39 \\
\hline $05 / 09 / 07$ & 3,000 & 2.68 & 15.61 & 0 & 489.5 & 35.6 & 42.02 & 12.9 & 0 & 16 & 12.39 \\
\hline $06 / 27 / 07$ & 1,400 & 2.20 & 11.91 & 0 & 476.5 & 30.35 & 74 & 2 & 40 & 12.96 & 9.13 \\
\hline 07/04/07 & 240 & 2.64 & 17.32 & 0.02 & 477 & 32.14 & 51.59 & 0 & 8 & 12.82 & 11.27 \\
\hline $07 / 11 / 07$ & 2,200 & 3.49 & 2.44 & 0.13 & 479 & 32.62 & 64 & 0.9 & 6 & 12.89 & 0.84 \\
\hline 07/18/07 & 5,000 & 2.87 & 1.92 & 0 & 544.5 & 49.33 & 104 & 10.5 & 16 & 9.03 & 8.64 \\
\hline $07 / 25 / 07$ & 22,000 & 1.43 & 3.07 & 0.41 & 431 & 24.73 & 36 & 15.2 & 20 & 11.47 & 11.16 \\
\hline $08 / 15 / 07$ & 9,000 & 4.62 & 0.59 & 0 & 450 & 35.86 & 67.67 & 1.5 & 0 & 10.42 & 8.73 \\
\hline $09 / 19 / 07$ & 22,000 & 4.06 & 0.63 & - & - & 21.25 & - & 0.9 & - & - & 7.89 \\
\hline Average & 16,418 & 2.68 & 5.95 & 0.04 & 503.8 & 40.74 & 97.67 & 3.5 & 19 & 11.24 & 7.69 \\
\hline $\begin{array}{l}\text { Standard } \\
\text { Deviation }\end{array}$ & 23,912 & 1.06 & 5.19 & 0.11 & 86.9 & 13.58 & 47.15 & 4.7 & 16 & 4.12 & 3.68 \\
\hline
\end{tabular}




\begin{tabular}{|c|c|c|c|c|c|c|c|c|c|c|c|}
\hline \multicolumn{12}{|c|}{ Table B-5 SF Effluent (SF 2-5 Phragmites/Typha) } \\
\hline $\begin{array}{l}\text { Sampling } \\
\text { Date }\end{array}$ & $\begin{array}{l}\text { Fecal Coliform } \\
\text { (NMP/100 mL) }\end{array}$ & $\begin{array}{l}\text { Phosphorus } \\
\text { (mg/L) }\end{array}$ & $\begin{array}{l}\text { Nitrates } \\
(\mathrm{mg} / \mathrm{L})\end{array}$ & $\begin{array}{c}\text { Nitrites } \\
(\mathrm{mg} / \mathrm{L})\end{array}$ & $\begin{array}{c}\text { TDS } \\
(\mathrm{mg} / \mathrm{L})\end{array}$ & $\begin{array}{c}\text { BOD } \\
(\mathrm{mg} / \mathrm{L})\end{array}$ & $\begin{array}{c}\text { COD } \\
(\mathrm{mg} / \mathrm{L})\end{array}$ & $\begin{array}{c}\text { Oil \& Grease } \\
(\mathrm{mg} / \mathrm{L})\end{array}$ & $\begin{array}{c}\text { TSS } \\
(\mathrm{mg} / \mathrm{L})\end{array}$ & $\begin{array}{c}\text { Total Nitrogen } \\
(\mathrm{mg} / \mathrm{L})\end{array}$ & $\underset{(\mathrm{mg} / \mathrm{L})}{\operatorname{Ammonium}}$ \\
\hline $11 / 29 / 06$ & 800 & 0.02 & 1.25 & 0.00 & 270.00 & 15.39 & 79.37 & 1.00 & 4.00 & 13.59 & 0.15 \\
\hline $12 / 19 / 06$ & 140 & 0.14 & 1.25 & 0.00 & 300.00 & 11.93 & 22.36 & 2.00 & 0.00 & 5.52 & 0.13 \\
\hline $01 / 08 / 07$ & 300 & 2.08 & 2.01 & 0.00 & 438.50 & 9.64 & 32.52 & 0.20 & 4.00 & 2.76 & 0.14 \\
\hline 01/17/07 & - & 1.37 & 1.99 & 0.00 & 490.00 & 13.85 & 54.69 & 0.80 & 0.00 & 5.52 & 0.17 \\
\hline $01 / 24 / 07$ & 900 & 2.01 & 2.65 & 0.00 & 528.50 & 25.06 & 171.30 & 0.50 & 4.00 & 5.52 & 0.17 \\
\hline $01 / 30 / 07$ & 13 & 5.97 & 1.95 & 2.44 & 0.00 & 520.00 & 46.78 & 55.44 & 0.80 & 4.00 & 2.76 \\
\hline $02 / 07 / 07$ & 13 & 1.85 & 2.08 & 0.00 & 554.00 & 7.70 & 55.56 & 1.90 & 8.00 & 5.52 & 2.71 \\
\hline 03/13/07 & 500 & 2.64 & 5.09 & 0.00 & 846.50 & 24.52 & 81.97 & 1.40 & 10.00 & 5.93 & - \\
\hline $04 / 24 / 07$ & 500 & 2.85 & 2.44 & 0.00 & 750.00 & 21.13 & 108.53 & 1.00 & 10.00 & 7.70 & 0.29 \\
\hline $05 / 09 / 07$ & 130 & 2.72 & 5.17 & 0.00 & 480.00 & 13.45 & 25.21 & 6.90 & 0.00 & 5.43 & 1.04 \\
\hline $06 / 27 / 07$ & 240 & 0.50 & 6.05 & 0.00 & 425.00 & 20.01 & 88.00 & 1.80 & 0.00 & 2.01 & 0.12 \\
\hline $07 / 04 / 07$ & 170 & 0.66 & 3.13 & 0.00 & 294.50 & 27.07 & 41.67 & 1.40 & 0.00 & 1.77 & 0.00 \\
\hline $07 / 11 / 07$ & 1100 & 0.70 & 2.67 & 0.00 & 178.50 & 22.09 & 176.00 & 1.70 & 1.00 & 2.03 & 0.19 \\
\hline 07/18/07 & 140 & 1.10 & 0.00 & 0.00 & 291.00 & 37.29 & 76.00 & 0.70 & 6.00 & 2.10 & 0.16 \\
\hline $07 / 25 / 07$ & 500 & 2.22 & 1.03 & 0.60 & 356.00 & 15.35 & 48.00 & 1.00 & 0.00 & 4.61 & 3.76 \\
\hline 08/15/07 & 500 & 2.33 & 0.47 & 0.00 & 311.00 & 20.52 & 60.15 & 1.30 & 0.00 & 1.02 & 0.35 \\
\hline 09/19/07 & 2200 & 2.26 & 0.55 & - & - & 10.77 & - & 0.00 & - & - & 0.00 \\
\hline Average & 509 & 1.85 & 2.34 & 0.19 & 407.1 & 47.99 & 73.01 & 4.6 & 3 & 4.69 & 0.76 \\
\hline $\begin{array}{l}\text { Standard } \\
\text { Deviation }\end{array}$ & 553 & 1.41 & 1.71 & 0.62 & 207.6 & 121.87 & 45.81 & 13.2 & 4 & 3.06 & 1.19 \\
\hline
\end{tabular}




\begin{tabular}{|c|c|c|c|c|c|c|c|c|c|c|c|}
\hline \multicolumn{12}{|c|}{ Table B-6 SSF Effluent (SF 3-6 Thalia/Thalia) } \\
\hline $\begin{array}{l}\text { Sampling } \\
\text { Date }\end{array}$ & $\begin{array}{l}\text { Fecal Coliform } \\
\text { (NMP/100 mL) }\end{array}$ & $\begin{array}{l}\text { Phosphorus } \\
\text { (mg/L) }\end{array}$ & $\begin{array}{l}\text { Nitrates } \\
(\mathrm{mg} / \mathrm{L})\end{array}$ & $\begin{array}{c}\text { Nitrites } \\
(\mathrm{mg} / \mathrm{L})\end{array}$ & $\begin{array}{c}\text { TDS } \\
(\mathrm{mg} / \mathrm{L})\end{array}$ & $\begin{array}{c}\text { BOD } \\
(\mathrm{mg} / \mathrm{L})\end{array}$ & $\begin{array}{c}\text { COD } \\
(\mathrm{mg} / \mathrm{L})\end{array}$ & $\begin{array}{c}\text { Oil \& Grease } \\
(\mathrm{mg} / \mathrm{L})\end{array}$ & $\begin{array}{c}\text { TSS } \\
(\mathrm{mg} / \mathrm{L})\end{array}$ & $\begin{array}{l}\text { Total Nitrogen } \\
(\mathrm{mg} / \mathrm{L})\end{array}$ & $\underset{(\mathrm{mg} / \mathrm{L})}{\operatorname{Ammonium}}$ \\
\hline $11 / 29 / 06$ & 11000 & 0.47 & 1.63 & 0.00 & 330.00 & 18.05 & 101.19 & 0.40 & 10.00 & 10.87 & 0.09 \\
\hline $12 / 19 / 06$ & 280 & 0.30 & 2.24 & 0.23 & 350.00 & 11.27 & 24.39 & 3.80 & 0.00 & 8.28 & 0.09 \\
\hline $01 / 08 / 07$ & 1400 & 2.86 & 2.68 & 0.00 & 469.50 & 13.14 & 48.78 & 0.40 & 6.00 & 8.28 & 0.07 \\
\hline $01 / 17 / 07$ & - & 2.60 & 1.67 & 0.00 & 519.00 & 10.95 & 46.88 & 0.90 & 0.00 & 2.76 & 0.47 \\
\hline $01 / 24 / 07$ & 900 & 1.82 & 2.53 & 0.00 & 540.00 & 25.29 & 212.96 & 0.40 & 8.00 & 5.52 & 0.30 \\
\hline $01 / 30 / 07$ & 240 & 2.16 & 2.51 & 0.00 & 518.50 & 39.71 & 50.40 & 0.80 & 6.00 & 5.52 & 0.34 \\
\hline $02 / 07 / 07$ & 8 & 2.02 & 2.56 & 0.00 & 574.50 & 12.15 & 79.37 & 0.50 & 4.00 & 5.52 & 1.50 \\
\hline 03/13/07 & 5000 & 2.07 & 5.64 & 0.00 & 766.00 & 44.45 & 459.02 & 1.90 & 80.00 & 13.31 & - \\
\hline $04 / 24 / 07$ & 5000 & 0.23 & 4.62 & 0.00 & 621.00 & 30.09 & 224.81 & 5.40 & 82.00 & 14.27 & 1.97 \\
\hline $05 / 09 / 07$ & 2400 & 0.38 & 6.38 & 0.34 & 374.50 & 25.51 & 33.61 & 8.60 & 16.00 & 6.34 & 0.19 \\
\hline 06/27/07 & 500 & 0.95 & 3.12 & 0.00 & 377.50 & 13.58 & 92.00 & 0.90 & 16.00 & 2.73 & 0.13 \\
\hline $07 / 04 / 07$ & 16000 & 1.07 & 1.92 & 0.00 & 325.50 & 25.31 & 103.17 & 3.90 & 12.00 & 1.89 & 0.45 \\
\hline $07 / 11 / 07$ & 170 & 0.84 & 1.75 & 1.09 & 250.00 & 21.56 & 24.00 & 1.10 & 0.00 & 2.50 & 0.67 \\
\hline 07/18/07 & 5000 & 1.84 & 1.58 & 0.00 & 373.00 & 45.36 & 96.00 & 4.40 & 16.00 & 3.55 & 1.64 \\
\hline $07 / 25 / 07$ & 5000 & 2.36 & 1.75 & 0.00 & 361.50 & 13.31 & 60.00 & 1.30 & 0.00 & 4.72 & 428.00 \\
\hline 08/15/07 & 1300 & 2.44 & 0.78 & 0.00 & 353.50 & 23.79 & 413.53 & 8.10 & 0.00 & 1.63 & 0.90 \\
\hline 09/19/07 & 0 & 2.57 & 1.56 & - & - & 8.73 & - & 1.50 & - & - & 0.00 \\
\hline Average & 3,387 & 1.59 & 2.64 & 0.10 & 444.0 & 22.49 & 129.38 & 2.6 & 16 & 6.11 & 27.30 \\
\hline $\begin{array}{l}\text { Standard } \\
\text { Deviation }\end{array}$ & 4,502 & 0.91 & 1.52 & 0.28 & 135.6 & 11.76 & 133.58 & 2.7 & 26 & 3.95 & 106.85 \\
\hline
\end{tabular}




\begin{tabular}{|c|c|c|c|c|c|c|c|c|c|c|c|}
\hline \multicolumn{12}{|c|}{ Table B-7 SSF Effluent (SF 4-7 Brachiaria/Cyperus) } \\
\hline $\begin{array}{l}\text { Sampling } \\
\text { Date }\end{array}$ & $\begin{array}{l}\text { Fecal Coliform } \\
\text { (NMP/100 mL) }\end{array}$ & $\begin{array}{c}\text { Phosphorus } \\
(\mathrm{mg} / \mathrm{L})\end{array}$ & $\begin{array}{c}\text { Nitrates } \\
(\mathrm{mg} / \mathrm{L})\end{array}$ & $\begin{array}{l}\text { Nitrites } \\
(\mathrm{mg} / \mathrm{L})\end{array}$ & $\begin{array}{c}\text { TDS } \\
(\mathrm{mg} / \mathrm{L})\end{array}$ & $\begin{array}{c}\text { BOD } \\
(\mathrm{mg} / \mathrm{L})\end{array}$ & $\begin{array}{c}\text { COD } \\
(\mathrm{mg} / \mathrm{L})\end{array}$ & $\begin{array}{c}\text { Oil \& } \\
\text { Grease } \\
(\mathrm{mg} / \mathrm{L})\end{array}$ & $\begin{array}{c}\text { TSS } \\
(\mathrm{mg} / \mathrm{L})\end{array}$ & $\begin{array}{c}\text { Total Nitrogen } \\
(\mathrm{mg} / \mathrm{L})\end{array}$ & $\begin{array}{c}\text { Ammonium } \\
(\mathrm{mg} / \mathrm{L})\end{array}$ \\
\hline $11 / 29 / 06$ & 800 & 0.00 & 0.95 & 0.00 & 270.00 & 12.82 & 71.43 & 0.20 & 0.00 & 10.87 & 0.11 \\
\hline $12 / 19 / 06$ & 220 & 0.21 & 1.15 & 0.00 & 330.00 & 9.93 & 28.46 & 3.40 & 0.00 & 7.59 & 0.57 \\
\hline $01 / 08 / 07$ & 300 & 2.64 & 2.54 & 0.00 & 453.00 & 11.14 & 36.59 & 0.20 & 6.00 & 2.76 & 0.04 \\
\hline $01 / 17 / 07$ & - & 3.03 & 1.73 & 0.00 & 553.00 & 10.49 & 50.78 & 0.40 & 0.00 & 5.52 & 0.67 \\
\hline $01 / 24 / 07$ & 2400 & 3.49 & 2.82 & 0.00 & 533.50 & 72.99 & 194.44 & 0.50 & 4.00 & 2.76 & 0.28 \\
\hline 01/30/07 & 1100 & 2.98 & 2.65 & 0.00 & 515.00 & 36.48 & 70.56 & 1.20 & 4.00 & 2.76 & 0.23 \\
\hline $02 / 07 / 07$ & 800 & 2.90 & 2.15 & 0.00 & 605.00 & 48.25 & 119.05 & 0.20 & 4.00 & 6.90 & 0.40 \\
\hline 03/13/07 & 8000 & 3.06 & 5.03 & 0.00 & 740.50 & 21.62 & 90.16 & 2.50 & 20.00 & 7.19 & - \\
\hline 04/24/07 & 3000 & 3.02 & 4.47 & 0.00 & 702.50 & 20.39 & 131.78 & 9.80 & 8.00 & 11.29 & 0.35 \\
\hline 05/09/07 & 2400 & 2.43 & 11.98 & 0.25 & 506.00 & 27.28 & 33.61 & 6.70 & 8.00 & 6.64 & 0.07 \\
\hline 06/27/07 & 80 & 0.68 & 2.76 & 0.00 & 374.50 & 10.32 & 52.00 & 0.60 & 0.00 & 2.26 & 0.16 \\
\hline 07/04/07 & 5000 & 0.60 & 12.21 & 0.00 & 360.50 & 27.31 & 37.70 & 5.90 & 4.00 & 5.48 & 0.56 \\
\hline 07/11/07 & 5000 & 0.60 & 5.08 & 1.06 & 312.00 & 5.73 & 40.00 & 0.70 & 0.00 & 4.20 & 0.14 \\
\hline 07/18/07 & 1100 & 1.55 & 0.00 & 0.00 & 314.50 & 37.16 & 56.00 & 1.50 & 4.00 & 3.92 & 0.14 \\
\hline $07 / 25 / 07$ & 300 & 1.80 & 6.61 & 0.35 & 309.50 & 12.15 & 140.00 & 0.20 & 6.00 & 6.67 & 5.70 \\
\hline 08/15/07 & 130 & 0.94 & 0.53 & 0.06 & 270.00 & 28.25 & 45.11 & 2.10 & 0.00 & 1.80 & 1.19 \\
\hline 09/19/07 & 0 & 1.92 & 0.70 & - & - & 5.50 & - & 1.10 & - & - & 0.00 \\
\hline Average & 1,914 & 1.87 & 3.73 & 0.11 & 446.8 & 23.40 & 74.85 & 2.2 & 4 & 5.54 & 0.66 \\
\hline $\begin{array}{l}\text { Standard } \\
\text { Deviation }\end{array}$ & 2,307 & 1.17 & 3.63 & 0.27 & 152.1 & 17.76 & 47.79 & 2.8 & 5 & 2.89 & 1.38 \\
\hline
\end{tabular}




\begin{tabular}{|c|c|c|c|c|c|c|c|c|c|c|c|c|c|c|}
\hline \multicolumn{15}{|c|}{ Table B-8 Summary of SSF Effluent Concentration and \% Residual - Average and Standard Deviation } \\
\hline \multirow[t]{2}{*}{ Parameter } & \multicolumn{2}{|c|}{ SSF 1} & \multicolumn{4}{|c|}{ SSF 1-2 } & \multicolumn{4}{|c|}{ SSF 1-3 } & \multicolumn{4}{|c|}{ SSF 1-4 } \\
\hline & $\begin{array}{c}\text { AVE } \\
\text { Conc. } \\
(\mathrm{mg} / \mathrm{L})\end{array}$ & $\begin{array}{c}\text { Conc. } \\
\text { SD } \\
(\mathrm{mg} / \mathrm{L})\end{array}$ & $\begin{array}{c}\text { AVE } \\
\text { Conc. } \\
\text { (mg/L) }\end{array}$ & $\begin{array}{c}\text { Conc. } \\
\text { SD } \\
(\mathrm{mg} / \mathrm{L})\end{array}$ & $\begin{array}{c}\text { AVE } \\
\% \\
\text { Res. }\end{array}$ & $\begin{array}{c}\text { \% Res. } \\
\text { SD } \\
(\mathrm{mg} / \mathrm{L})\end{array}$ & $\begin{array}{c}\text { AVE } \\
\text { Conc. } \\
(\mathrm{mg} / \mathrm{L})\end{array}$ & $\begin{array}{c}\text { Conc. } \\
\text { SD } \\
(\mathrm{mg} / \mathrm{L})\end{array}$ & $\begin{array}{c}\text { AVE } \\
\% \\
\text { Res. }\end{array}$ & $\begin{array}{c}\% \\
\text { Res. } \\
\text { SD }\end{array}$ & $\begin{array}{c}\text { AVE } \\
\text { Conc. } \\
(\mathrm{mg} / \mathrm{L})\end{array}$ & $\begin{array}{c}\text { Conc. } \\
\text { SD } \\
(\mathrm{mg} / \mathrm{L})\end{array}$ & $\begin{array}{c}\text { AVE } \\
\% \\
\text { Res. }\end{array}$ & $\begin{array}{c}\% \\
\text { Res. } \\
\text { SD }\end{array}$ \\
\hline $\begin{array}{c}\text { Fecal } \\
\text { Coliform }\end{array}$ & 183,525 & 388,002 & 11,445 & 13,337 & $15 \%$ & $16 \%$ & 27,254 & 20,680 & $63 \%$ & $77 \%$ & 16,418 & 23,912 & $18 \%$ & $22 \%$ \\
\hline Phosphorus & 1.8 & 1.3 & 2.9 & 1.0 & $246 \%$ & $207 \%$ & 2.7 & 1.3 & $197 \%$ & $160 \%$ & 2.7 & 1.1 & $224 \%$ & $203 \%$ \\
\hline Nitrates & 5.1 & 3.9 & 5.6 & 6.2 & $136 \%$ & $149 \%$ & 5.2 & 5.4 & $129 \%$ & $130 \%$ & 6.0 & 5.2 & $139 \%$ & $114 \%$ \\
\hline Nitrites** & 0.1 & 0.2 & 0.0 & 0.1 & $0 \%$ & $1 \%$ & 0.3 & 1.0 & $36 \%$ & $134 \%$ & 0.0 & 0.1 & $0 \%$ & $1 \%$ \\
\hline TDS & 517.0 & 133.9 & 524.9 & 103.1 & $109 \%$ & $52 \%$ & 523.3 & 93.6 & $108 \%$ & $44 \%$ & 503.8 & 86.9 & $105 \%$ & $45 \%$ \\
\hline BOD & 66.7 & 27.3 & 50.5 & 21.0 & $81 \%$ & $46 \%$ & 53.4 & 35.2 & $78 \%$ & $28 \%$ & 40.7 & 13.6 & $64 \%$ & $19 \%$ \\
\hline COD & 269.7 & 194.6 & 116.7 & 53.5 & $53 \%$ & $28 \%$ & 141.6 & 61.7 & $62 \%$ & $26 \%$ & 97.7 & 47.1 & $45 \%$ & $26 \%$ \\
\hline $\begin{array}{c}\text { Oil \& } \\
\text { Grease }\end{array}$ & 5.9 & 5.5 & 3.7 & 3.0 & $132 \%$ & $188 \%$ & 3.2 & 3.2 & $111 \%$ & $170 \%$ & 3.5 & 4.7 & $152 \%$ & $306 \%$ \\
\hline TSS & 162.1 & 145.9 & 20.3 & 27.1 & $14 \%$ & $16 \%$ & 24.8 & 32.4 & $16 \%$ & $19 \%$ & 19.1 & 16.4 & $12 \%$ & $12 \%$ \\
\hline $\begin{array}{c}\text { Total } \\
\text { Nitrogen }\end{array}$ & 19.7 & 9.1 & 13.0 & 4.7 & $95 \%$ & $96 \%$ & 12.9 & 4.1 & $88 \%$ & $82 \%$ & 11.2 & 4.1 & $79 \%$ & $72 \%$ \\
\hline Ammonium & 7.3 & 1.4 & 8.0 & 4.7 & $590 \%$ & $910 \%$ & 8.6 & 3.7 & $683 \%$ & $1160 \%$ & 7.7 & 3.7 & $690 \%$ & $1073 \%$ \\
\hline
\end{tabular}




\begin{tabular}{|c|c|c|c|c|c|c|c|c|c|c|c|c|}
\hline \multicolumn{13}{|c|}{ Table B-9 Summary of SF Effluent Concentration and \% Residual - Average and Standard Deviation } \\
\hline \multirow[t]{2}{*}{ Parameter } & \multicolumn{4}{|c|}{ SF 2-5 } & \multicolumn{4}{|c|}{ SF 3-6 } & \multicolumn{4}{|c|}{ SF 4-7 } \\
\hline & $\begin{array}{c}\text { AVE } \\
\text { Conc. } \\
\text { (mg/L) }\end{array}$ & $\begin{array}{c}\text { Conc. SD } \\
(\mathrm{mg} / \mathrm{L})\end{array}$ & $\begin{array}{c}\text { AVE } \\
\text { \% Res. }\end{array}$ & $\begin{array}{c}\text { \% Res. } \\
\text { SD } \\
(\mathrm{mg} / \mathrm{L})\end{array}$ & $\begin{array}{c}\text { AVE } \\
\text { Conc. } \\
\text { (mg/L) }\end{array}$ & $\begin{array}{c}\text { Conc. SD } \\
(\mathrm{mg} / \mathrm{L})\end{array}$ & $\begin{array}{c}\text { AVE \% } \\
\text { Res. }\end{array}$ & $\begin{array}{c}\text { \% Res. } \\
\text { SD }\end{array}$ & $\begin{array}{c}\text { AVE } \\
\text { Conc. } \\
(\mathrm{mg} / \mathrm{L})\end{array}$ & $\begin{array}{c}\text { Conc. SD } \\
(\mathrm{mg} / \mathrm{L})\end{array}$ & $\begin{array}{c}\text { AVE \% } \\
\text { Res. }\end{array}$ & $\begin{array}{c}\% \\
\text { Res. } \\
\text { SD }\end{array}$ \\
\hline $\begin{array}{c}\text { Fecal } \\
\text { Coliform }\end{array}$ & 509.1 & 552.9 & $14 \%$ & $20 \%$ & $3,387.4$ & $4,502.2$ & $50 \%$ & $110 \%$ & $1,914.4$ & $2,307.0$ & $298 \%$ & $722 \%$ \\
\hline Phosphorus & 1.8 & 1.4 & $65 \%$ & $55 \%$ & 1.6 & 0.9 & $88 \%$ & $113 \%$ & 1.9 & 1.2 & $78 \%$ & $54 \%$ \\
\hline Nitrates & 2.3 & 1.7 & $82 \%$ & $67 \%$ & 2.6 & 1.5 & $171 \%$ & $400 \%$ & 3.7 & 3.6 & $75 \%$ & $60 \%$ \\
\hline Nitrites** & 0.2 & 0.6 & $12 \%$ & $46 \%$ & 0.1 & 0.3 & $260 \%$ & $971 \%$ & 0.1 & 0.3 & $64 \%$ & $217 \%$ \\
\hline TDS & 407.1 & 207.6 & $81 \%$ & $21 \%$ & 444.0 & 135.6 & $85 \%$ & $17 \%$ & 446.8 & 152.1 & $87 \%$ & $19 \%$ \\
\hline BOD & 48.0 & 121.9 & $101 \%$ & $229 \%$ & 22.5 & 11.8 & $51 \%$ & $24 \%$ & 23.4 & 17.8 & $59 \%$ & $42 \%$ \\
\hline COD & 73.0 & 45.8 & $90 \%$ & $84 \%$ & 129.4 & 133.6 & $130 \%$ & $203 \%$ & 74.9 & 47.8 & $93 \%$ & $86 \%$ \\
\hline $\begin{array}{c}\text { Oil \& } \\
\text { Grease }\end{array}$ & 4.6 & 13.2 & $612 \%$ & $2227 \%$ & 2.6 & 2.7 & $161 \%$ & $224 \%$ & 2.2 & 2.8 & $214 \%$ & $599 \%$ \\
\hline TSS & 3.0 & 3.7 & $27 \%$ & $40 \%$ & 16.0 & 26.1 & $2824 \%$ & $5937 \%$ & 4.3 & 5.1 & $28 \%$ & $35 \%$ \\
\hline $\begin{array}{c}\text { Total } \\
\text { Nitrogen }\end{array}$ & 4.7 & 3.1 & $39 \%$ & $26 \%$ & 6.1 & 3.9 & $51 \%$ & $36 \%$ & 5.5 & 2.9 & $59 \%$ & $60 \%$ \\
\hline Ammonium & 0.8 & 1.2 & $9 \%$ & $13 \%$ & 27.3 & 106.9 & $272 \%$ & $1053 \%$ & 0.7 & 1.4 & $9 \%$ & $13 \%$ \\
\hline
\end{tabular}




\begin{tabular}{|c|c|c|c|c|c|c|c|c|c|c|c|c|c|c|}
\hline \multirow{3}{*}{ Parameter } & \multicolumn{14}{|c|}{ Table B-10 Summary of CW System Effluent Concentration and \% Residual - Average and Standard Deviation } \\
\hline & \multicolumn{2}{|c|}{ SSF 1} & \multicolumn{4}{|c|}{ SSF 1-2-5 } & \multicolumn{4}{|c|}{ SSF 1-3-6 } & \multicolumn{4}{|c|}{ SSF 1-4-7 } \\
\hline & $\begin{array}{c}\text { AVE } \\
\text { Conc. } \\
\text { (mg/L) }\end{array}$ & $\begin{array}{c}\text { Conc. } \\
\text { SD } \\
(\mathrm{mg} / \mathrm{L})\end{array}$ & $\begin{array}{c}\text { AVE } \\
\text { Conc. } \\
(\mathrm{mg} / \mathrm{L})\end{array}$ & $\begin{array}{c}\text { Conc. } \\
\text { SD } \\
(\mathrm{mg} / \mathrm{L})\end{array}$ & $\begin{array}{c}\text { AVE } \\
\% \\
\text { Res. }\end{array}$ & $\begin{array}{c}\text { \% Res. } \\
\text { SD } \\
(\mathrm{mg} / \mathrm{L})\end{array}$ & $\begin{array}{c}\text { AVE } \\
\text { Conc. } \\
(\mathrm{mg} / \mathrm{L})\end{array}$ & $\begin{array}{c}\text { Conc. } \\
\text { SD } \\
(\mathrm{mg} / \mathrm{L})\end{array}$ & $\begin{array}{c}\text { AVE } \\
\% \\
\text { Res. }\end{array}$ & $\begin{array}{c}\% \\
\text { Res. } \\
\text { SD }\end{array}$ & $\begin{array}{c}\text { AVE } \\
\text { Conc. } \\
\text { (mg/L) }\end{array}$ & $\begin{array}{c}\text { Conc. } \\
\text { SD } \\
(\mathrm{mg} / \mathrm{L})\end{array}$ & $\begin{array}{c}\text { AVE } \\
\% \\
\text { Res. }\end{array}$ & $\begin{array}{c}\% \\
\text { Res. } \\
\text { SD }\end{array}$ \\
\hline $\begin{array}{c}\text { Fecal } \\
\text { Coliform }\end{array}$ & 183,525 & 388,002 & 509.1 & 552.9 & $1 \%$ & $2 \%$ & $3,387.4$ & $4,502.2$ & $44 \%$ & $166 \%$ & $1,914.4$ & $2,307.0$ & $15 \%$ & $52 \%$ \\
\hline Phosphorus & 1.8 & 1.3 & 1.8 & 1.4 & $168 \%$ & $154 \%$ & 1.6 & 0.9 & $144 \%$ & $147 \%$ & 1.9 & 1.2 & $163 \%$ & $126 \%$ \\
\hline Nitrates & 5.1 & 3.9 & 2.3 & 1.7 & $57 \%$ & $26 \%$ & 2.6 & 1.5 & $76 \%$ & $59 \%$ & 3.7 & 3.6 & $95 \%$ & $95 \%$ \\
\hline Nitrites** & 0.1 & 0.2 & 0.2 & 0.6 & $0 \%$ & $0 \%$ & 0.1 & 0.3 & $0 \%$ & $0 \%$ & 0.1 & 0.3 & $0 \%$ & $0 \%$ \\
\hline TDS & 517.0 & 133.9 & 407.1 & 207.6 & $94 \%$ & $68 \%$ & 444.0 & 135.6 & $94 \%$ & $51 \%$ & 446.8 & 152.1 & $95 \%$ & $60 \%$ \\
\hline BOD & 66.7 & 27.3 & 48.0 & 121.9 & $67 \%$ & $145 \%$ & 22.5 & 11.8 & $38 \%$ & $21 \%$ & 23.4 & 17.8 & $38 \%$ & $26 \%$ \\
\hline COD & 269.7 & 194.6 & 73.0 & 45.8 & $40 \%$ & $44 \%$ & 129.4 & 133.6 & $56 \%$ & $59 \%$ & 74.9 & 47.8 & $35 \%$ & $24 \%$ \\
\hline $\begin{array}{c}\text { Oil \& } \\
\text { Grease }\end{array}$ & 5.9 & 5.5 & 4.6 & 13.2 & $280 \%$ & $950 \%$ & 2.6 & 2.7 & $89 \%$ & $124 \%$ & 2.2 & 2.8 & $42 \%$ & $49 \%$ \\
\hline TSS & 162.1 & 145.9 & 3.0 & 3.7 & $2 \%$ & $2 \%$ & 16.0 & 26.1 & $9 \%$ & $11 \%$ & 4.3 & 5.1 & $3 \%$ & $4 \%$ \\
\hline $\begin{array}{c}\text { Total } \\
\text { Nitrogen }\end{array}$ & 19.7 & 9.1 & 4.7 & 3.1 & $37 \%$ & $49 \%$ & 6.1 & 3.9 & $41 \%$ & $45 \%$ & 5.5 & 2.9 & $36 \%$ & $27 \%$ \\
\hline Ammonium & 7.3 & 1.4 & 0.8 & 1.2 & $55 \%$ & $103 \%$ & 27.3 & 106.9 & $164 \%$ & $484 \%$ & 0.7 & 1.4 & $43 \%$ & $100 \%$ \\
\hline
\end{tabular}




\begin{tabular}{|c|c|c|c|c|c|c|c|c|c|c|c|c|c|c|}
\hline \multicolumn{15}{|c|}{ Table B-11 Fecal Coliform (FC) Seasonal Percent Residual } \\
\hline \multirow[t]{2}{*}{ Season } & \multirow[b]{2}{*}{ Date } & \multirow{2}{*}{$\begin{array}{c}\text { FC Point } \\
1 \text { Conc. } \\
\text { (NMP/100 } \\
\text { mL) }\end{array}$} & \multicolumn{2}{|c|}{ FC Point 1-2 } & \multicolumn{2}{|c|}{ FC Point 1-3 } & \multicolumn{2}{|c|}{ FC Point 1-4 } & \multicolumn{2}{|c|}{ FC Point 2-5 } & \multicolumn{2}{|c|}{ FC Point 3-6 } & \multicolumn{2}{|c|}{ FC Point 4-7 } \\
\hline & & & $\begin{array}{c}\text { Conc. } \\
(\mathrm{NMP} / 100 \\
\text { mL) }\end{array}$ & $\begin{array}{c}\% \\
\text { Res. }\end{array}$ & $\begin{array}{c}\text { Conc. } \\
\text { (NMP/100 } \\
\text { mL) }\end{array}$ & \% Res. & $\begin{array}{c}\text { Conc. } \\
\text { (NMP/100 } \\
\text { mL) }\end{array}$ & $\begin{array}{c}\% \\
\text { Res. }\end{array}$ & $\begin{array}{c}\text { Conc. } \\
(\mathrm{NMP} / 100 \\
\text { mL) }\end{array}$ & $\begin{array}{c}\% \\
\text { Res. }\end{array}$ & $\begin{array}{c}\text { Conc. } \\
(\mathrm{NMP} / 100 \\
\text { mL) }\end{array}$ & $\begin{array}{c}\% \\
\text { Res. }\end{array}$ & $\begin{array}{c}\text { Conc. } \\
\text { (NMP/100 } \\
\text { mL) }\end{array}$ & $\begin{array}{c}\% \\
\text { Res. }\end{array}$ \\
\hline \multirow{11}{*}{$\begin{array}{l}\text { Wet } \\
\text { (May } \\
\text { to } \\
\text { Nov) }\end{array}$} & $11 / 29 / 06$ & 300,000 & 50,000 & $17 \%$ & 50,000 & $17 \%$ & 90,000 & $30 \%$ & 800 & $2 \%$ & 11,000 & $22 \%$ & 800 & $1 \%$ \\
\hline & $5 / 9 / 07$ & 300,000 & 240 & $0.1 \%$ & 17,000 & $5.7 \%$ & 3,000 & $1.0 \%$ & 130 & $54 \%$ & 2,400 & $14 \%$ & 2,400 & $80 \%$ \\
\hline & $6 / 27 / 07$ & 50,000 & 30,000 & $60 \%$ & 13,000 & $26 \%$ & 1,400 & $3 \%$ & 240 & $1 \%$ & 500 & $4 \%$ & 80 & $6 \%$ \\
\hline & $7 / 4 / 07$ & 2,400 & 240 & $10 \%$ & 5,000 & $208 \%$ & 240 & $10 \%$ & 170 & $71 \%$ & 16,000 & $320 \%$ & 5,000 & $2083 \%$ \\
\hline & $7 / 11 / 07$ & 50,000 & 9,000 & $18 \%$ & 30,000 & $60 \%$ & 2,200 & $4 \%$ & 1,100 & $12 \%$ & 170 & $1 \%$ & 5,000 & $227 \%$ \\
\hline & $7 / 18 / 07$ & 140,000 & 2,400 & $2 \%$ & 30,000 & $21 \%$ & 5,000 & $4 \%$ & 140 & $6 \%$ & 5,000 & $17 \%$ & 1,100 & $22 \%$ \\
\hline & $7 / 25 / 07$ & 30,000 & 9,000 & $30 \%$ & 80,000 & $267 \%$ & 22,000 & $73 \%$ & 500 & $6 \%$ & 5,000 & $6 \%$ & 300 & $1 \%$ \\
\hline & $8 / 15 / 07$ & 90,000 & 3,000 & $3 \%$ & 16,000 & $18 \%$ & 9,000 & $10 \%$ & 500 & $17 \%$ & 1,300 & $8 \%$ & 130 & $1 \%$ \\
\hline & $9 / 19 / 07$ & 50,000 & 11,000 & $22 \%$ & 50,000 & $100 \%$ & 22,000 & $44 \%$ & - & - & 0 & $0 \%$ & 0 & $0 \%$ \\
\hline & AVE & 112,489 & 12,764 & $18 \%$ & 32,333 & $80 \%$ & 17,204 & $20 \%$ & 448 & $21 \%$ & 4,597 & $44 \%$ & 1,646 & $269 \%$ \\
\hline & SD & 113,039 & 16,701 & $19 \%$ & 23,796 & $95 \%$ & 28,561 & $25 \%$ & 353 & $27 \%$ & 5,529 & $104 \%$ & 2,042 & $684 \%$ \\
\hline \multirow{10}{*}{$\begin{array}{l}\text { Dry } \\
\text { (Dec to } \\
\text { April) }\end{array}$} & $12 / 19 / 06$ & 50,000 & 16,000 & $32 \%$ & 50,000 & $100 \%$ & 11,000 & $22 \%$ & 140 & $1 \%$ & 280 & $1 \%$ & 220 & $2 \%$ \\
\hline & $1 / 8 / 07$ & 24,000 & 3,000 & $13 \%$ & 24,000 & $100 \%$ & 800 & $3 \%$ & 300 & $10 \%$ & 1,400 & $6 \%$ & 300 & $38 \%$ \\
\hline & $1 / 17 / 07$ & - & - & - & - & - & - & - & - & - & - & - & - & - \\
\hline & $1 / 24 / 07$ & 90,000 & 16,000 & $18 \%$ & 16,000 & $18 \%$ & 50,000 & $56 \%$ & 900 & $6 \%$ & 900 & $6 \%$ & 2,400 & $5 \%$ \\
\hline & $1 / 30 / 07$ & 30,000 & 110 & $0.4 \%$ & 70 & $0.2 \%$ & 50 & $0.2 \%$ & 13 & $12 \%$ & 240 & $343 \%$ & 1,100 & $2200 \%$ \\
\hline & $2 / 7 / 07$ & 50,000 & 130 & $0.3 \%$ & 17,000 & $34.0 \%$ & 3,000 & $6.0 \%$ & 13 & $10 \%$ & 8 & $0 \%$ & 800 & $27 \%$ \\
\hline & $3 / 13 / 07$ & 80,000 & 17,000 & $21 \%$ & 22,000 & $28 \%$ & 13,000 & $16 \%$ & 500 & $3 \%$ & 5,000 & $23 \%$ & 8,000 & $62 \%$ \\
\hline & $4 / 24 / 07$ & $1,600,000$ & 16,000 & $1 \%$ & 16,000 & $1 \%$ & 30,000 & $2 \%$ & 500 & $3 \%$ & 5,000 & $31 \%$ & 3,000 & $10 \%$ \\
\hline & AVE & 274,857 & 9,749 & $12 \%$ & 20,724 & $40.1 \%$ & 15,407 & $15 \%$ & 338 & $6 \%$ & 1,833 & $58 \%$ & 2,260 & $335 \%$ \\
\hline & SD & 584,829 & 8,173 & $12 \%$ & 15,025 & $42.8 \%$ & 18,430 & $20 \%$ & 322 & $4 \%$ & 2,214 & $126 \%$ & 2,738 & $823 \%$ \\
\hline Notes & \multicolumn{14}{|c|}{$\begin{array}{l}\text { - AVE }=\text { average; } S D=\text { standard deviation; Res. }=\text { Residual } \\
\text { - Point } 1=\text { SSF Influent, Point } 1-2=\text { Phragmites, Point } 1-3=\text { Thalia; } \text { Point } 1-4=\text { Brachiaria.; } \\
\text { Point } 2-5=\text { Phragmites } / \text { Typha; } \text { Point } 3-6=\text { Thalia/Thalia } ; \text { Point } 4-7=\text { Brachiaria } / \text { Cyperus } \\
\text { - Shaded cells indicates N.D. (non-detectable); }-=\text { data not available } \\
\text { - Average } \% \text { residual marked with a square around is greater than } 90 \% \text {; therefore, no further analysis was performed. }\end{array}$} \\
\hline
\end{tabular}




\begin{tabular}{|c|c|c|c|c|c|c|c|c|c|c|c|c|c|c|}
\hline \multicolumn{15}{|c|}{ Table B-12 Total Phosphorus (TP) Seasonal Percent Residual } \\
\hline \multirow[t]{2}{*}{ Season } & \multirow{2}{*}{ Date } & \multirow{2}{*}{$\begin{array}{c}\text { TP Point } 1 \\
\text { Conc. (mg/L) }\end{array}$} & \multicolumn{2}{|c|}{ TP Point 1-2 } & \multicolumn{2}{|c|}{ TP Point 1-3 } & \multicolumn{2}{|c|}{ TP Point 1-4 } & \multicolumn{2}{|c|}{ TP Point 2-5 } & \multicolumn{2}{|c|}{ TP Point 3-6 } & \multicolumn{2}{|c|}{ TP Point 4-7 } \\
\hline & & & $\begin{array}{l}\text { Conc. } \\
\text { (mg/L) }\end{array}$ & $\begin{array}{c}\% \\
\text { Res. }\end{array}$ & $\begin{array}{l}\text { Conc. } \\
\text { (mg/L) }\end{array}$ & $\begin{array}{c}\% \\
\text { Res. }\end{array}$ & $\begin{array}{l}\text { Conc. } \\
\text { (mg/L) }\end{array}$ & $\begin{array}{c}\% \\
\text { Res. }\end{array}$ & $\begin{array}{l}\text { Conc. } \\
\text { (mg/L) }\end{array}$ & $\begin{array}{c}\% \\
\text { Res. }\end{array}$ & $\begin{array}{l}\text { Conc. } \\
\text { (mg/L) }\end{array}$ & $\begin{array}{c}\% \\
\text { Res. }\end{array}$ & $\begin{array}{l}\text { Conc. } \\
\text { (mg/L) }\end{array}$ & $\begin{array}{c}\% \\
\text { Res. }\end{array}$ \\
\hline \multirow{11}{*}{$\begin{array}{l}\text { Wet } \\
\text { (May } \\
\text { to } \\
\text { Nov) }\end{array}$} & $11 / 29 / 06$ & 2.40 & 2.22 & $93 \%$ & 2.16 & $90 \%$ & 2.15 & $90 \%$ & 0.02 & $1 \%$ & 0.47 & $22 \%$ & 0.00 & $0 \%$ \\
\hline & $5 / 9 / 07$ & 1.15 & 3.59 & $314 \%$ & 2.97 & $259 \%$ & 2.68 & $234 \%$ & 2.72 & $76 \%$ & 0.38 & $13 \%$ & 2.43 & $91 \%$ \\
\hline & $6 / 27 / 07$ & 3.58 & 2.81 & $78 \%$ & 3.56 & $99 \%$ & 2.20 & $61 \%$ & 0.50 & $18 \%$ & 0.95 & $27 \%$ & 0.68 & $31 \%$ \\
\hline & $7 / 4 / 07$ & 3.41 & 2.97 & $87 \%$ & 3.47 & $102 \%$ & 2.64 & $78 \%$ & 0.66 & $22 \%$ & 1.07 & $31 \%$ & 0.60 & $23 \%$ \\
\hline & $7 / 11 / 07$ & 2.41 & 3.63 & $151 \%$ & 3.71 & $154 \%$ & 3.49 & $145 \%$ & 0.70 & $19 \%$ & 0.84 & $23 \%$ & 0.60 & $17 \%$ \\
\hline & $7 / 18 / 07$ & 0.48 & 2.73 & $569 \%$ & 1.60 & $333 \%$ & 2.87 & $598 \%$ & 1.10 & $40 \%$ & 1.84 & $115 \%$ & 1.55 & $54 \%$ \\
\hline & $7 / 25 / 07$ & 0.53 & 2.02 & $381 \%$ & 0.47 & $89 \%$ & 1.43 & $270 \%$ & 2.22 & $110 \%$ & 2.36 & $502 \%$ & 1.80 & $126 \%$ \\
\hline & $8 / 15 / 07$ & 0.57 & 4.73 & $831 \%$ & 4.06 & $714 \%$ & 4.62 & $812 \%$ & 2.33 & $49 \%$ & 2.44 & $60 \%$ & 0.94 & $20 \%$ \\
\hline & $9 / 19 / 07$ & 4.69 & 4.28 & $91 \%$ & 4.25 & $91 \%$ & 4.06 & $87 \%$ & 2.26 & $53 \%$ & 2.57 & $61 \%$ & 1.92 & $47 \%$ \\
\hline & AVE & 2.13 & 3.22 & $288 \%$ & 2.92 & $214 \%$ & 2.90 & $264 \%$ & 1.39 & $43 \%$ & 1.44 & $95 \%$ & 1.17 & $45 \%$ \\
\hline & SD & 1.55 & 0.91 & $265 \%$ & 1.26 & $207 \%$ & 1.00 & $266 \%$ & 0.99 & $34 \%$ & 0.87 & $156 \%$ & 0.79 & $40 \%$ \\
\hline \multirow{10}{*}{$\begin{array}{c}\text { Dry } \\
\text { (Dec to } \\
\text { April) }\end{array}$} & $12 / 19 / 06$ & 0.91 & 0.42 & $46 \%$ & 0.29 & $32 \%$ & 0.28 & $31 \%$ & 0.14 & $33 \%$ & 0.30 & $103 \%$ & 0.21 & $72 \%$ \\
\hline & $1 / 8 / 07$ & 1.30 & 4.02 & $309 \%$ & 3.92 & $301 \%$ & 3.87 & $297 \%$ & 2.08 & $52 \%$ & 2.86 & $73 \%$ & 2.64 & $68 \%$ \\
\hline & $1 / 17 / 07$ & 1.42 & 2.37 & $167 \%$ & 2.08 & $147 \%$ & 2.89 & $204 \%$ & 1.37 & $58 \%$ & 2.60 & $125 \%$ & 3.03 & $105 \%$ \\
\hline & $1 / 24 / 07$ & 1.17 & 2.09 & $178 \%$ & 2.00 & $170 \%$ & 1.76 & $150 \%$ & 2.01 & $96 \%$ & 1.82 & $91 \%$ & 3.49 & $198 \%$ \\
\hline & $1 / 30 / 07$ & 1.35 & 2.46 & $182 \%$ & 2.23 & $165 \%$ & 2.04 & $151 \%$ & 5.97 & $243 \%$ & 2.16 & $97 \%$ & 2.98 & $146 \%$ \\
\hline & $2 / 7 / 07$ & 1.36 & 2.57 & $189 \%$ & 2.57 & $189 \%$ & 2.31 & $170 \%$ & 1.85 & $72 \%$ & 2.02 & $78 \%$ & 2.90 & $125 \%$ \\
\hline & $3 / 13 / 07$ & 3.31 & 3.54 & $107 \%$ & 3.53 & $107 \%$ & 3.58 & $108 \%$ & 2.64 & $75 \%$ & 2.07 & $58 \%$ & 3.06 & $86 \%$ \\
\hline & $4 / 24 / 07$ & 0.83 & 3.36 & $404 \%$ & 2.62 & $315 \%$ & 2.70 & $325 \%$ & 2.85 & $85 \%$ & 0.23 & $9 \%$ & 3.02 & $112 \%$ \\
\hline & AVE & 1.46 & 2.60 & $198 \%$ & 2.41 & $178 \%$ & 2.43 & $179 \%$ & 2.36 & $89 \%$ & 1.76 & $79 \%$ & 2.67 & $114 \%$ \\
\hline & SD & 0.78 & 1.11 & $112 \%$ & 1.10 & $94 \%$ & 1.13 & $96 \%$ & 1.68 & $65 \%$ & 0.98 & $35 \%$ & 1.02 & $43 \%$ \\
\hline Notes & \multicolumn{14}{|c|}{$\begin{array}{l}\text { - AVE }=\text { average; } S D=\text { standard deviation; Res. }=\text { Residual } \\
\text { - Point } 1=S S F \text { Influent, Point } 1-2=\text { Phragmites, Point } 1-3=\text { Thalia; } \text { Point } 1-4=\text { Brachiaria.; } \\
\text { Point } 2-5=\text { Phragmites } / \text { Typha; Point } 3-6=\text { Thalia/Thalia; } \text { Point } 4-7=\text { Brachiaria/Cyperus } \\
\text { - Shaded cells indicates N.D. (non-detectable); }-=\text { data not available } \\
\text { - Average } \% \text { residual marked with a square around is greater than } 90 \% \text {; therefore, no further analysis was performed. }\end{array}$} \\
\hline
\end{tabular}




\section{Table B-13 Nitrates Seasonal Percent Residual}

\begin{tabular}{|c|c|c|c|c|c|c|c|c|c|c|c|c|c|c|}
\hline \multicolumn{15}{|c|}{ Table B-13 Nitrates Seasonal Percent Residual } \\
\hline \multirow[t]{2}{*}{ Season } & \multirow{2}{*}{ Date } & \multirow{2}{*}{$\begin{array}{l}\text { Nitrates Point } 1 \\
\text { Conc. }(\mathrm{mg} / \mathrm{L})\end{array}$} & \multicolumn{2}{|c|}{ Nitrates Point 1-2 } & \multicolumn{2}{|c|}{ Nitrates Point 1-3 } & \multicolumn{2}{|c|}{ Nitrates Point 1-4 } & \multicolumn{2}{|c|}{ Nitrates Point 2-5 } & \multicolumn{2}{|c|}{ Nitrates Point 3-6 } & \multicolumn{2}{|c|}{ Nitrates Point 4-7 } \\
\hline & & & $\begin{array}{l}\text { Conc. } \\
\text { (mg/L) }\end{array}$ & $\begin{array}{l}\% \\
\text { Res. }\end{array}$ & $\begin{array}{l}\text { Conc. } \\
\text { (mg/L) }\end{array}$ & $\begin{array}{l}\% \\
\text { Res. }\end{array}$ & $\begin{array}{l}\text { Conc. } \\
(\mathrm{mg} / \mathrm{L})\end{array}$ & $\begin{array}{c}\% \\
\text { Res. }\end{array}$ & $\begin{array}{l}\text { Conc. } \\
\text { (mg/L) }\end{array}$ & $\begin{array}{l}\% \\
\text { Res. }\end{array}$ & $\begin{array}{l}\text { Conc. } \\
(\mathrm{mg} / \mathrm{L})\end{array}$ & $\begin{array}{l}\% \\
\text { Res. }\end{array}$ & $\begin{array}{l}\text { Conc. } \\
\text { (mg/L) }\end{array}$ & $\begin{array}{l}\% \\
\text { Res. }\end{array}$ \\
\hline \multirow{11}{*}{$\begin{array}{l}\text { Wet } \\
\text { (May } \\
\text { to } \\
\text { Nov) }\end{array}$} & $11 / 29 / 06$ & 2.23 & 2.09 & $94 \%$ & 2.55 & $114 \%$ & 2.71 & $122 \%$ & 1.25 & $60 \%$ & 1.63 & $64 \%$ & 0.95 & $35 \%$ \\
\hline & $5 / 9 / 07$ & 5.66 & 19.56 & $346 \%$ & 14.62 & $258 \%$ & 15.61 & $276 \%$ & 5.17 & $26 \%$ & 6.38 & $44 \%$ & 11.98 & $77 \%$ \\
\hline & $6 / 27 / 07$ & 15.43 & 2.71 & $18 \%$ & 5.47 & $35 \%$ & 11.91 & $77 \%$ & 6.05 & $223 \%$ & 3.12 & $57 \%$ & 2.76 & $23 \%$ \\
\hline & $7 / 4 / 07$ & 3.48 & 19.68 & $566 \%$ & 19.68 & $566 \%$ & 17.32 & $498 \%$ & 3.13 & $16 \%$ & 1.92 & $10 \%$ & 12.21 & $70 \%$ \\
\hline & $7 / 11 / 07$ & 4.57 & 3.19 & $70 \%$ & 5.54 & $121 \%$ & 2.44 & $53 \%$ & 2.67 & $84 \%$ & 1.75 & $32 \%$ & 5.08 & $208 \%$ \\
\hline & $7 / 18 / 07$ & 1.35 & 0.66 & $49 \%$ & 2.67 & $198 \%$ & 1.92 & $142 \%$ & 0.00 & $0 \%$ & 1.58 & $59 \%$ & 0.00 & $0 \%$ \\
\hline & $7 / 25 / 07$ & 2.59 & 0.97 & $37 \%$ & 1.57 & $61 \%$ & 3.07 & $119 \%$ & 1.03 & $106 \%$ & 1.75 & $111 \%$ & 6.61 & $215 \%$ \\
\hline & $8 / 15 / 07$ & 8.47 & 0.25 & $3 \%$ & 0.59 & $7 \%$ & 0.59 & $7 \%$ & 0.47 & $188 \%$ & 0.78 & $132 \%$ & 0.53 & $90 \%$ \\
\hline & 9/19/07 & 0.57 & 0.78 & $137 \%$ & 0.72 & $126 \%$ & 0.63 & $111 \%$ & 0.55 & $71 \%$ & 1.56 & $217 \%$ & 0.70 & $111 \%$ \\
\hline & AVE & 4.93 & 5.54 & $146 \%$ & 5.93 & $165 \%$ & 6.24 & $156 \%$ & 2.54 & $97 \%$ & 2.27 & $81 \%$ & 4.54 & $92 \%$ \\
\hline & SD & 4.61 & 8.04 & $188 \%$ & 6.72 & $169 \%$ & 6.72 & $148 \%$ & 2.13 & $74 \%$ & 1.65 & $63 \%$ & 4.83 & $76 \%$ \\
\hline \multirow{10}{*}{$\begin{array}{c}\text { Dry } \\
\text { (Dec to } \\
\text { April) }\end{array}$} & $12 / 19 / 06$ & 3.82 & 2.76 & $72 \%$ & 1.89 & $49 \%$ & 3.43 & $90 \%$ & 1.25 & $45 \%$ & 2.24 & $119 \%$ & 1.15 & $34 \%$ \\
\hline & $1 / 8 / 07$ & 11.31 & 10.71 & $95 \%$ & 8.29 & $73 \%$ & 7.19 & $64 \%$ & 2.01 & $19 \%$ & 2.68 & $32 \%$ & 2.54 & $35 \%$ \\
\hline & $1 / 17 / 07$ & 3.08 & 2.16 & $70 \%$ & 2.06 & $67 \%$ & 2.67 & $87 \%$ & 1.99 & $92 \%$ & 1.67 & $81 \%$ & 1.73 & $65 \%$ \\
\hline & $1 / 24 / 07$ & 3.01 & 10.28 & $342 \%$ & 4.21 & $140 \%$ & 6.72 & $223 \%$ & 2.65 & $26 \%$ & 2.53 & $60 \%$ & 2.82 & $42 \%$ \\
\hline & $1 / 30 / 07$ & 2.94 & 6.11 & $208 \%$ & 4.02 & $137 \%$ & 6.20 & $211 \%$ & 1.95 & $32 \%$ & 2.51 & $62 \%$ & 2.65 & $43 \%$ \\
\hline & $2 / 7 / 07$ & 3.46 & 3.65 & $105 \%$ & 3.63 & $105 \%$ & 3.10 & $90 \%$ & 2.08 & $57 \%$ & 2.56 & $71 \%$ & 2.15 & $69 \%$ \\
\hline & $3 / 13 / 07$ & 9.12 & 8.00 & $88 \%$ & 11.25 & $123 \%$ & 11.98 & $131 \%$ & 5.09 & $64 \%$ & 5.64 & $50 \%$ & 5.03 & $42 \%$ \\
\hline & $4 / 24 / 07$ & 5.59 & 1.20 & $21 \%$ & 0.27 & $5 \%$ & 3.68 & $66 \%$ & 2.44 & $203 \%$ & 4.62 & $1711 \%$ & 4.47 & $121 \%$ \\
\hline & AVE & 5.29 & 5.61 & $125 \%$ & 4.45 & $87 \%$ & 5.62 & $120 \%$ & 2.43 & $67 \%$ & 3.06 & $273 \%$ & 2.82 & $56 \%$ \\
\hline & SD & 3.21 & 3.72 & $102 \%$ & 3.62 & $47 \%$ & 3.12 & $63 \%$ & 1.15 & $60 \%$ & 1.35 & $582 \%$ & 1.32 & $29 \%$ \\
\hline Notes & \multicolumn{14}{|c|}{$\begin{array}{l}\text { - AVE = average; } S D=\text { standard deviation; Res. }=\text { Residual } \\
\text { - Point } 1=S S F \text { Influent, Point } 1-2=\text { Phragmites, Point } 1-3=\text { Thalia; } \text { Point } 1-4=\text { Brachiaria.; } \\
\text { Point } 2-5=\text { Phragmites/Typha; Point } 3-6=\text { Thalia/Thalia; Point } 4-7=\text { Brachiaria/Cyperus } \\
\text { - Shaded cells indicates N.D. (non-detectable); }-=\text { data not available } \\
\text { - Average } \% \text { residual marked with a square around is greater than } 90 \% \text {; therefore, no further analysis was performed. }\end{array}$} \\
\hline
\end{tabular}




\begin{tabular}{|c|c|c|c|c|c|c|c|c|c|c|c|c|c|c|}
\hline \multicolumn{15}{|c|}{ Table B-14 Nitrites Seasonal Percent Residual } \\
\hline \multirow[t]{2}{*}{ Season } & \multirow{2}{*}{ Date } & \multirow{2}{*}{$\begin{array}{l}\text { Nitrites Point } \\
1 \text { Conc. } \\
\text { (mg/L) }\end{array}$} & \multicolumn{2}{|c|}{ Nitrites Point 1-2 } & \multicolumn{2}{|c|}{ Nitrites Point 1-3 } & \multicolumn{2}{|c|}{ Nitrites Point 1-4 } & \multicolumn{2}{|c|}{ Nitrites Point 2-5 } & \multicolumn{2}{|c|}{ Nitrites Point 3-6 } & \multicolumn{2}{|c|}{ Nitrites Point 4-7 } \\
\hline & & & $\begin{array}{l}\text { Conc. } \\
\text { (mg/L) }\end{array}$ & $\begin{array}{c}\% \\
\text { Res. }\end{array}$ & $\begin{array}{l}\text { Conc. } \\
\text { (mg/L) }\end{array}$ & \% Res. & $\begin{array}{l}\text { Conc. } \\
\text { (mg/L) }\end{array}$ & \% Res. & $\begin{array}{c}\text { Conc. } \\
\text { (mg/L) }\end{array}$ & $\%$ Res. & $\begin{array}{l}\text { Conc. } \\
\text { (mg/L) }\end{array}$ & \% Res. & $\begin{array}{l}\text { Conc. } \\
\text { (mg/L) }\end{array}$ & \% Res. \\
\hline \multirow{11}{*}{$\begin{array}{l}\text { Wet } \\
\text { (May } \\
\text { to } \\
\text { Nov) }\end{array}$} & $11 / 29 / 06$ & 0.00 & 0.00 & $0.0 \%$ & 0.00 & $0.0 \%$ & 0.00 & $0.0 \%$ & 0.00 & $0 \%$ & 0.00 & $0 \%$ & 0.00 & $0 \%$ \\
\hline & $5 / 9 / 07$ & 0.00 & 0.00 & $0.0 \%$ & 0.00 & $0.0 \%$ & 0.00 & $0.0 \%$ & 0.00 & $0 \%$ & 0.34 & $>1000 \%$ & 0.25 & $>1000 \%$ \\
\hline & $6 / 27 / 07$ & 0.00 & 0.00 & $0.0 \%$ & 0.00 & $0.0 \%$ & 0.00 & $0.0 \%$ & 0.00 & $0 \%$ & 0.00 & $0 \%$ & 0.00 & $0 \%$ \\
\hline & $7 / 4 / 07$ & 0.80 & 0.02 & $2.5 \%$ & 4.00 & $500.0 \%$ & 0.02 & $2.5 \%$ & 0.00 & $0 \%$ & 0.00 & $0 \%$ & 0.00 & $0 \%$ \\
\hline & $7 / 11 / 07$ & 0.00 & 0.03 & $0.0 \%$ & 0.03 & $>1000 \%$ & 0.13 & $>1000 \%$ & 0.00 & $0 \%$ & 1.09 & $3633 \%$ & 1.06 & $815 \%$ \\
\hline & $7 / 18 / 07$ & 0.00 & 0.00 & $0.0 \%$ & 0.00 & $0.0 \%$ & 0.00 & $0.0 \%$ & 0.00 & $0 \%$ & 0.00 & $0 \%$ & 0.00 & $0 \%$ \\
\hline & $7 / 25 / 07$ & 0.00 & 0.34 & $>100 \%$ & 0.61 & $>1000 \%$ & 0.41 & $>1000 \%$ & 0.60 & $176 \%$ & 0.00 & $0 \%$ & 0.35 & $85 \%$ \\
\hline & $8 / 15 / 07$ & 0.00 & 0.00 & $0.0 \%$ & 0.00 & $0.0 \%$ & 0.00 & $0.0 \%$ & 0.00 & $0 \%$ & 0.00 & $0 \%$ & 0.06 & $>1000 \%$ \\
\hline & $9 / 19 / 07$ & - & - & - & - & - & - & - & - & - & - & - & - & - \\
\hline & AVE & 0.10 & 0.05 & $0.4 \%$ & 0.58 & $83.3 \%$ & 0.07 & $0.4 \%$ & 0.08 & $22 \%$ & 0.18 & $519 \%$ & 0.22 & $150 \%$ \\
\hline & SD & 0.28 & 0.12 & $0.9 \%$ & 1.40 & $204.1 \%$ & 0.14 & $1.0 \%$ & 0.21 & $62 \%$ & 0.39 & $1373 \%$ & 0.37 & $328 \%$ \\
\hline \multirow{10}{*}{$\begin{array}{l}\text { Dry } \\
\text { (Dec to } \\
\text { April) }\end{array}$} & $12 / 19 / 06$ & 0.00 & 0.00 & $0.0 \%$ & 0.00 & $0.0 \%$ & 0.00 & $0.0 \%$ & 0.00 & $0 \%$ & 0.23 & $>1000 \%$ & 0.00 & $0 \%$ \\
\hline & $1 / 8 / 07$ & 0.00 & 0.00 & $0.0 \%$ & 0.00 & $0.0 \%$ & 0.00 & $0.0 \%$ & 0.00 & $0 \%$ & 0.00 & $0 \%$ & 0.00 & $0 \%$ \\
\hline & $1 / 17 / 07$ & 0.00 & 0.00 & $0.0 \%$ & 0.00 & $0.0 \%$ & 0.00 & $0.0 \%$ & 0.00 & $0 \%$ & 0.00 & $0 \%$ & 0.00 & $0 \%$ \\
\hline & $1 / 24 / 07$ & 0.00 & 0.00 & $0.0 \%$ & 0.00 & $0.0 \%$ & 0.00 & $0.0 \%$ & 0.00 & $0 \%$ & 0.00 & $0 \%$ & 0.00 & $0 \%$ \\
\hline & $1 / 30 / 07$ & 0.00 & 0.00 & $0.0 \%$ & 0.00 & $0.0 \%$ & 0.00 & $0.0 \%$ & 2.44 & $>1000 \%$ & 0.00 & $0 \%$ & 0.00 & $0 \%$ \\
\hline & $2 / 7 / 07$ & 0.00 & 0.00 & $0.0 \%$ & 0.00 & $0.0 \%$ & 0.00 & $0.0 \%$ & 0.00 & $0 \%$ & 0.00 & $0 \%$ & 0.00 & $0 \%$ \\
\hline & $3 / 13 / 07$ & 0.00 & 0.00 & $0.0 \%$ & 0.00 & $0.0 \%$ & 0.00 & $0.0 \%$ & 0.00 & $0 \%$ & 0.00 & $0 \%$ & 0.00 & $0 \%$ \\
\hline & $4 / 24 / 07$ & 0.00 & 0.00 & $0.0 \%$ & 0.00 & $0.0 \%$ & 0.00 & $0.0 \%$ & 0.00 & $0 \%$ & 0.00 & $0 \%$ & 0.00 & $0 \%$ \\
\hline & AVE & 0.00 & 0.00 & $0 \%$ & 0.00 & $0 \%$ & 0.00 & $0 \%$ & 0.31 & $0 \%$ & 0.03 & $0 \%$ & 0.00 & $0 \%$ \\
\hline & SD & 0.00 & 0.00 & $0 \%$ & 0.00 & $0 \%$ & 0.00 & $0 \%$ & 0.86 & $0 \%$ & 0.08 & $0 \%$ & 0.00 & $0 \%$ \\
\hline \multicolumn{15}{|l|}{ Notes } \\
\hline
\end{tabular}




\begin{tabular}{|c|c|c|c|c|c|c|c|c|c|c|c|c|c|c|}
\hline \multicolumn{15}{|c|}{ Table B-15 Total Dissolved Solids (TDS) Seasonal Percent Residual } \\
\hline \multirow[t]{2}{*}{ Season } & \multirow{2}{*}{ Date } & \multirow{2}{*}{$\begin{array}{c}\text { TDS Point } 1 \\
\text { Conc. }(\mathrm{mg} / \mathrm{L})\end{array}$} & \multicolumn{2}{|c|}{ TDS Point 1-2 } & \multicolumn{2}{|c|}{ TDS Point 1-3 } & \multicolumn{2}{|c|}{ TDS Point 1-4 } & \multicolumn{2}{|c|}{ TDS Point 2-5 } & \multicolumn{2}{|c|}{ TDS Point 3-6 } & \multicolumn{2}{|c|}{ TDS Point 4-7 } \\
\hline & & & $\begin{array}{l}\text { Conc. } \\
\text { (mg/L) }\end{array}$ & $\begin{array}{c}\% \\
\text { Res. }\end{array}$ & $\begin{array}{l}\text { Conc. } \\
\text { (mg/L) }\end{array}$ & $\begin{array}{c}\% \\
\text { Res. }\end{array}$ & $\begin{array}{l}\text { Conc. } \\
\text { (mg/L) }\end{array}$ & $\begin{array}{c}\% \\
\text { Res. }\end{array}$ & $\begin{array}{l}\text { Conc. } \\
\text { (mg/L) }\end{array}$ & $\begin{array}{c}\% \\
\text { Res. }\end{array}$ & $\begin{array}{l}\text { Conc. } \\
\text { (mg/L) }\end{array}$ & $\begin{array}{c}\% \\
\text { Res. }\end{array}$ & $\begin{array}{l}\text { Conc. } \\
\text { (mg/L) }\end{array}$ & $\begin{array}{c}\% \\
\text { Res. }\end{array}$ \\
\hline \multirow{11}{*}{$\begin{array}{l}\text { Wet } \\
\text { (May } \\
\text { to } \\
\text { Nov) }\end{array}$} & $11 / 29 / 06$ & 320.0 & 330.0 & $103 \%$ & 330.0 & $103 \%$ & 320.0 & $100 \%$ & 270.00 & $82 \%$ & 330.00 & $100 \%$ & 270.00 & $84 \%$ \\
\hline & $5 / 9 / 07$ & 484.5 & 518.5 & $107 \%$ & 520.0 & $107 \%$ & 489.5 & $101 \%$ & 480.00 & $93 \%$ & 374.50 & $72 \%$ & 506.00 & $103 \%$ \\
\hline & $6 / 27 / 07$ & 617.5 & 516.0 & $84 \%$ & 507.5 & $82 \%$ & 476.5 & $77 \%$ & 425.00 & $82 \%$ & 377.50 & $74 \%$ & 374.50 & $79 \%$ \\
\hline & $7 / 4 / 07$ & 606.0 & 554.5 & $92 \%$ & 551.5 & $91 \%$ & 477.0 & $79 \%$ & 294.50 & $53 \%$ & 325.50 & $59 \%$ & 360.50 & $76 \%$ \\
\hline & $7 / 11 / 07$ & 606.5 & 578.5 & $95 \%$ & 547.5 & $90 \%$ & 479.0 & $79 \%$ & 178.50 & $31 \%$ & 250.00 & $46 \%$ & 312.00 & $65 \%$ \\
\hline & $7 / 18 / 07$ & 474.5 & 411.0 & $87 \%$ & 422.5 & $89 \%$ & 544.5 & $115 \%$ & 291.00 & $71 \%$ & 373.00 & $88 \%$ & 314.50 & $58 \%$ \\
\hline & $7 / 25 / 07$ & 510.5 & 439.0 & $86 \%$ & 478.0 & $94 \%$ & 431.0 & $84 \%$ & 356.00 & $81 \%$ & 361.50 & $76 \%$ & 309.50 & $72 \%$ \\
\hline & $8 / 15 / 07$ & 563.0 & 459.5 & $82 \%$ & 471.0 & $84 \%$ & 450.0 & $80 \%$ & 311.00 & $68 \%$ & 353.50 & $75 \%$ & 270.00 & $60 \%$ \\
\hline & $9 / 19 / 07$ & - & - & - & - & - & - & - & - & - & - & - & - & - \\
\hline & AVE & 522.8 & 475.9 & $92 \%$ & 478.5 & $93 \%$ & 458.4 & $89 \%$ & 325.8 & $70 \%$ & 343.2 & $74 \%$ & 339.6 & $75 \%$ \\
\hline & SD & 99.7 & 82.2 & $9 \%$ & 73.6 & $9 \%$ & 64.9 & $14 \%$ & 93.9 & $20 \%$ & 42.5 & $17 \%$ & 76.8 & $15 \%$ \\
\hline \multirow{10}{*}{$\begin{array}{c}\text { Dry } \\
\text { (Dec to } \\
\text { April) }\end{array}$} & $12 / 19 / 06$ & 420.0 & 430.0 & $102 \%$ & 460.0 & $110 \%$ & 420.0 & $100 \%$ & 300.00 & $70 \%$ & 350.00 & $76 \%$ & 330.00 & $79 \%$ \\
\hline & $1 / 8 / 07$ & 450.0 & 535.0 & $119 \%$ & 522.0 & $116 \%$ & 542.5 & $121 \%$ & 438.50 & $82 \%$ & 469.50 & $90 \%$ & 453.00 & $84 \%$ \\
\hline & $1 / 17 / 07$ & 489.5 & 536.0 & $109 \%$ & 512.0 & $105 \%$ & 537.0 & $110 \%$ & 490.00 & $91 \%$ & 519.00 & $101 \%$ & 553.00 & $103 \%$ \\
\hline & $1 / 24 / 07$ & 522.0 & 528.0 & $101 \%$ & 528.5 & $101 \%$ & 502.0 & $96 \%$ & 528.50 & $100 \%$ & 540.00 & $102 \%$ & 533.50 & $106 \%$ \\
\hline & $1 / 30 / 07$ & 524.5 & 524.5 & $100 \%$ & 514.5 & $98 \%$ & 489.0 & $93 \%$ & 0.00 & $0 \%$ & 518.50 & $101 \%$ & 515.00 & $105 \%$ \\
\hline & $2 / 7 / 07$ & 645.5 & 598.0 & $93 \%$ & 645.5 & $100 \%$ & 590.5 & $91 \%$ & 554.00 & $93 \%$ & 574.50 & $89 \%$ & 605.00 & $102 \%$ \\
\hline & $3 / 13 / 07$ & 808.5 & 753.5 & $93 \%$ & 746.5 & $92 \%$ & 697.5 & $86 \%$ & 846.50 & $112 \%$ & 766.00 & $103 \%$ & 740.50 & $106 \%$ \\
\hline & $4 / 24 / 07$ & 229.5 & 686.5 & $299 \%$ & 615.5 & $268 \%$ & 615.0 & $268 \%$ & 750.00 & $109 \%$ & 621.00 & $101 \%$ & 702.50 & $114 \%$ \\
\hline & AVE & 511.2 & 573.9 & $127 \%$ & 568.1 & $124 \%$ & 549.2 & $121 \%$ & 558.2 & $94 \%$ & 544.8 & $95 \%$ & 554.1 & $100 \%$ \\
\hline & SD & 168.4 & 102.6 & $70 \%$ & 93.7 & $59 \%$ & 85.2 & $61 \%$ & 185.5 & $15 \%$ & 119.9 & $10 \%$ & 131.9 & $12 \%$ \\
\hline \multicolumn{15}{|l|}{ Notes } \\
\hline
\end{tabular}




\begin{tabular}{|c|c|c|c|c|c|c|c|c|c|c|c|c|c|c|}
\hline \multicolumn{15}{|c|}{ Table B-16 5-day Biological Oxygen Demand (BOD) $)$ Seasonal Percent Residual } \\
\hline \multirow[t]{2}{*}{ Season } & \multirow[b]{2}{*}{ Date } & \multirow{2}{*}{$\begin{array}{l}\text { BOD } 5 \text { Point } 1 \\
\text { Conc. }(\mathrm{mg} / \mathrm{L})\end{array}$} & \multicolumn{2}{|c|}{ BOD $_{5}$ Point 1-2 } & \multicolumn{2}{|c|}{ BOD 5 Point 1-3 } & \multicolumn{2}{|c|}{ BOD $_{5}$ Point 1-4 } & \multicolumn{2}{|c|}{ BOD5 Point 2-5 } & \multicolumn{2}{|c|}{ BOD $_{5}$ Point 3-6 } & \multicolumn{2}{|c|}{ BOD $_{5}$ Point 4-7 } \\
\hline & & & $\begin{array}{l}\text { Conc. } \\
\text { (mg/L) }\end{array}$ & $\begin{array}{c}\% \\
\text { Res. }\end{array}$ & $\begin{array}{l}\text { Conc. } \\
\text { (mg/L) }\end{array}$ & $\begin{array}{c}\% \\
\text { Res. }\end{array}$ & $\begin{array}{l}\text { Conc. } \\
\text { (mg/L) }\end{array}$ & $\begin{array}{c}\% \\
\text { Res. }\end{array}$ & $\begin{array}{l}\text { Conc. } \\
\text { (mg/L) }\end{array}$ & $\begin{array}{c}\% \\
\text { Res. }\end{array}$ & $\begin{array}{l}\text { Conc. } \\
\text { (mg/L) }\end{array}$ & $\begin{array}{c}\% \\
\text { Res. }\end{array}$ & $\begin{array}{l}\text { Conc. } \\
\text { (mg/L) }\end{array}$ & $\begin{array}{c}\% \\
\text { Res. }\end{array}$ \\
\hline \multirow{11}{*}{$\begin{array}{c}\text { Wet } \\
\text { (May } \\
\text { to } \\
\text { Nov) }\end{array}$} & $11 / 29 / 06$ & 72.43 & 44.76 & $62 \%$ & 41.29 & $57 \%$ & 39.23 & $54 \%$ & 15.39 & $34 \%$ & 18.05 & $44 \%$ & 12.82 & $33 \%$ \\
\hline & $5 / 9 / 07$ & 32.81 & 78.10 & $238 \%$ & 32.06 & $98 \%$ & 35.60 & $109 \%$ & 13.45 & $17 \%$ & 25.51 & $80 \%$ & 27.28 & $77 \%$ \\
\hline & $6 / 27 / 07$ & 63.27 & 32.60 & $52 \%$ & 31.02 & $49 \%$ & 30.35 & $48 \%$ & 20.01 & $61 \%$ & 13.58 & $44 \%$ & 10.32 & $34 \%$ \\
\hline & $7 / 4 / 07$ & 43.59 & 41.18 & $94 \%$ & 36.05 & $83 \%$ & 32.14 & $74 \%$ & 27.07 & $66 \%$ & 25.31 & $70 \%$ & 27.31 & $85 \%$ \\
\hline & $7 / 11 / 07$ & 43.16 & 28.45 & $66 \%$ & 25.74 & $60 \%$ & 32.62 & $76 \%$ & 22.09 & $78 \%$ & 21.56 & $84 \%$ & 5.73 & $18 \%$ \\
\hline & $7 / 18 / 07$ & 79.99 & 64.32 & $80 \%$ & 55.12 & $69 \%$ & 49.33 & $62 \%$ & 37.29 & $58 \%$ & 45.36 & $82 \%$ & 37.16 & $75 \%$ \\
\hline & $7 / 25 / 07$ & 42.64 & 16.93 & $40 \%$ & 27.06 & $63 \%$ & 24.73 & $58 \%$ & 15.35 & $91 \%$ & 13.31 & $49 \%$ & 12.15 & $49 \%$ \\
\hline & $8 / 15 / 07$ & 63.44 & 35.98 & $57 \%$ & 30.23 & $48 \%$ & 35.86 & $57 \%$ & 20.52 & $57 \%$ & 23.79 & $79 \%$ & 28.25 & $79 \%$ \\
\hline & $9 / 19 / 07$ & 42.92 & 13.67 & $32 \%$ & 21.33 & $50 \%$ & 21.25 & $50 \%$ & 10.77 & $79 \%$ & 8.73 & $41 \%$ & 5.50 & $26 \%$ \\
\hline & AVE & 53.81 & 39.55 & $80 \%$ & 33.32 & $64 \%$ & 33.46 & $65 \%$ & 20.22 & $60 \%$ & 21.69 & $64 \%$ & 18.50 & $53 \%$ \\
\hline & SD & 16.27 & 20.90 & $62 \%$ & 10.02 & $17 \%$ & 8.16 & $19 \%$ & 8.09 & $23 \%$ & 10.66 & $19 \%$ & 11.56 & $26 \%$ \\
\hline \multirow{10}{*}{$\begin{array}{l}\text { Dry } \\
\text { (Dec } \\
\text { to } \\
\text { April) }\end{array}$} & $12 / 19 / 06$ & 152.27 & 92.33 & $61 \%$ & 167.40 & $110 \%$ & 80.67 & $53 \%$ & 11.93 & $13 \%$ & 11.27 & $7 \%$ & 9.93 & $12 \%$ \\
\hline & $1 / 8 / 07$ & 80.04 & 66.31 & $83 \%$ & 48.24 & $60 \%$ & 45.11 & $56 \%$ & 9.64 & $15 \%$ & 13.14 & $27 \%$ & 11.14 & $25 \%$ \\
\hline & $1 / 17 / 07$ & 65.43 & 46.76 & $71 \%$ & 39.16 & $60 \%$ & 38.44 & $59 \%$ & 13.85 & $30 \%$ & 10.95 & $28 \%$ & 10.49 & $27 \%$ \\
\hline & $1 / 24 / 07$ & 80.55 & 62.16 & $77 \%$ & 66.78 & $83 \%$ & 44.33 & $55 \%$ & 25.06 & $40 \%$ & 25.29 & $38 \%$ & 72.99 & $165 \%$ \\
\hline & $1 / 30 / 07$ & 83.18 & 52.77 & $63 \%$ & 65.56 & $79 \%$ & 47.95 & $58 \%$ & 520.00 & $985 \%$ & 39.71 & $61 \%$ & 36.48 & $76 \%$ \\
\hline & $2 / 7 / 07$ & 67.90 & 56.36 & $83 \%$ & 93.65 & $138 \%$ & 36.36 & $54 \%$ & 7.70 & $14 \%$ & 12.15 & $13 \%$ & 48.25 & $133 \%$ \\
\hline & $3 / 13 / 07$ & 72.48 & 61.90 & $85 \%$ & 61.03 & $84 \%$ & 42.57 & $59 \%$ & 24.52 & $40 \%$ & 44.45 & $73 \%$ & 21.62 & $51 \%$ \\
\hline & $4 / 24 / 07$ & 48.20 & 63.83 & $132 \%$ & 66.03 & $137 \%$ & 55.99 & $116 \%$ & 21.13 & $33 \%$ & 30.09 & $46 \%$ & 20.39 & $36 \%$ \\
\hline & AVE & 81.26 & 62.80 & $82 \%$ & 75.98 & $94 \%$ & 48.93 & $64 \%$ & 79.23 & $146 \%$ & 23.38 & $36 \%$ & 28.91 & $66 \%$ \\
\hline & SD & 30.82 & 13.55 & $22 \%$ & 40.20 & $31 \%$ & 14.15 & $21 \%$ & 178.22 & $339 \%$ & 13.58 & $23 \%$ & 22.41 & $55 \%$ \\
\hline \multicolumn{15}{|l|}{ Notes } \\
\hline
\end{tabular}




\begin{tabular}{|c|c|c|c|c|c|c|c|c|c|c|c|c|c|c|}
\hline \multicolumn{15}{|c|}{ Table B-17 Chemical Oxygen Demand (COD) Seasonal Percent Residual } \\
\hline \multirow[t]{2}{*}{ Season } & \multirow[b]{2}{*}{ Date } & \multirow{2}{*}{$\begin{array}{l}\text { COD Point } 1 \\
\text { Conc. }(\mathrm{mg} / \mathrm{L})\end{array}$} & \multicolumn{2}{|c|}{ COD Point 1-2 } & \multicolumn{2}{|c|}{ COD Point 1-3 } & \multicolumn{2}{|c|}{ COD Point 1-4 } & \multicolumn{2}{|c|}{ COD Point 2-5 } & \multicolumn{2}{|c|}{ COD Point 3-6 } & \multicolumn{2}{|c|}{ COD Point 4-7 } \\
\hline & & & $\begin{array}{l}\text { Conc. } \\
\text { (mg/L) }\end{array}$ & $\begin{array}{c}\% \\
\text { Res. }\end{array}$ & $\begin{array}{l}\text { Conc. } \\
(\mathrm{mg} / \mathrm{L})\end{array}$ & $\begin{array}{c}\% \\
\text { Res. }\end{array}$ & $\begin{array}{l}\text { Conc. } \\
\text { (mg/L) }\end{array}$ & $\begin{array}{c}\% \\
\text { Res. }\end{array}$ & $\begin{array}{l}\text { Conc. } \\
\text { (mg/L) }\end{array}$ & $\begin{array}{c}\% \\
\text { Res. }\end{array}$ & $\begin{array}{l}\text { Conc. } \\
(\mathrm{mg} / \mathrm{L})\end{array}$ & $\begin{array}{c}\% \\
\text { Res. }\end{array}$ & $\begin{array}{l}\text { Conc. } \\
\text { (mg/L) }\end{array}$ & $\begin{array}{c}\% \\
\text { Res. }\end{array}$ \\
\hline \multirow{11}{*}{$\begin{array}{l}\text { Wet } \\
\text { (May } \\
\text { to } \\
\text { Nov) }\end{array}$} & $11 / 29 / 06$ & 223.21 & 128.97 & $58 \%$ & 158.73 & $71 \%$ & 148.81 & $67 \%$ & 79.37 & $62 \%$ & 101.19 & $64 \%$ & 71.43 & $48 \%$ \\
\hline & $5 / 9 / 07$ & 184.87 & 184.87 & $100 \%$ & 193.28 & $105 \%$ & 42.02 & $23 \%$ & 25.21 & $14 \%$ & 33.61 & $17 \%$ & 33.61 & $80 \%$ \\
\hline & $6 / 27 / 07$ & 216.00 & 80.00 & $37 \%$ & 144.00 & $67 \%$ & 74.00 & $34 \%$ & 88.00 & $110 \%$ & 92.00 & $64 \%$ & 52.00 & $70 \%$ \\
\hline & $7 / 4 / 07$ & 51.59 & 47.62 & $92 \%$ & 45.63 & $88 \%$ & 51.59 & $100 \%$ & 41.67 & $88 \%$ & 103.17 & $226 \%$ & 37.70 & $73 \%$ \\
\hline & $7 / 11 / 07$ & 96.00 & 56.00 & $58 \%$ & 56.00 & $58 \%$ & 64.00 & $67 \%$ & 176.00 & $314 \%$ & 24.00 & $43 \%$ & 40.00 & $63 \%$ \\
\hline & $7 / 18 / 07$ & 316.00 & 112.00 & $35 \%$ & 112.00 & $35 \%$ & 104.00 & $33 \%$ & 76.00 & $68 \%$ & 96.00 & $86 \%$ & 56.00 & $54 \%$ \\
\hline & $7 / 25 / 07$ & 180.00 & 20.00 & $11 \%$ & 92.00 & $51 \%$ & 36.00 & $20 \%$ & 48.00 & $240 \%$ & 60.00 & $65 \%$ & 140.00 & $389 \%$ \\
\hline & $8 / 15 / 07$ & 214.29 & 60.15 & $28 \%$ & 48.87 & $23 \%$ & 67.67 & $32 \%$ & 60.15 & $100 \%$ & 413.53 & $846 \%$ & 45.11 & $67 \%$ \\
\hline & 9/19/07 & - & - & - & - & - & - & - & - & - & - & - & - & - \\
\hline & AVE & 185.25 & 86.20 & $53 \%$ & 106.31 & $62 \%$ & 73.51 & $47 \%$ & 74.30 & $124 \%$ & 115.44 & $176 \%$ & 59.48 & $105 \%$ \\
\hline & SD & 81.23 & 53.09 & $31 \%$ & 55.40 & $27 \%$ & 37.04 & $28 \%$ & 46.18 & $101 \%$ & 124.37 & $278 \%$ & 34.69 & $115 \%$ \\
\hline \multirow{10}{*}{$\begin{array}{l}\text { Dry } \\
\text { (Dec to } \\
\text { April) }\end{array}$} & $12 / 19 / 06$ & 304.88 & 172.76 & $57 \%$ & 238.82 & $78 \%$ & 96.54 & $32 \%$ & 22.36 & $13 \%$ & 24.39 & $10 \%$ & 28.46 & $29 \%$ \\
\hline & $1 / 8 / 07$ & 142.28 & 130.08 & $91 \%$ & 113.82 & $80 \%$ & 109.76 & $77 \%$ & 32.52 & $25 \%$ & 48.78 & $43 \%$ & 36.59 & $33 \%$ \\
\hline & $1 / 17 / 07$ & 267.58 & 175.78 & $66 \%$ & 171.88 & $64 \%$ & 175.78 & $66 \%$ & 54.69 & $31 \%$ & 46.88 & $27 \%$ & 50.78 & $29 \%$ \\
\hline & $1 / 24 / 07$ & 222.22 & 106.48 & $48 \%$ & 175.93 & $79 \%$ & 180.56 & $81 \%$ & 171.30 & $161 \%$ & 212.96 & $121 \%$ & 194.44 & $108 \%$ \\
\hline & $1 / 30 / 07$ & 252.02 & 206.65 & $82 \%$ & 241.94 & $96 \%$ & 60.48 & $24 \%$ & 46.78 & $23 \%$ & 50.40 & $21 \%$ & 70.56 & $117 \%$ \\
\hline & $2 / 7 / 07$ & 324.80 & 114.17 & $35 \%$ & 127.95 & $39 \%$ & 102.36 & $32 \%$ & 55.56 & $49 \%$ & 79.37 & $62 \%$ & 119.05 & $116 \%$ \\
\hline & $3 / 13 / 07$ & 922.11 & 139.34 & $15 \%$ & 196.72 & $21 \%$ & 163.93 & $18 \%$ & 81.97 & $59 \%$ & 459.02 & $233 \%$ & 90.16 & $55 \%$ \\
\hline & $4 / 24 / 07$ & 397.29 & 131.78 & $33 \%$ & 147.29 & $37 \%$ & 85.27 & $21 \%$ & 108.53 & $82 \%$ & 224.81 & $153 \%$ & 131.78 & $155 \%$ \\
\hline & AVE & 354.15 & 147.13 & $53 \%$ & 176.79 & $62 \%$ & 121.84 & $44 \%$ & 71.71 & $55 \%$ & 143.33 & $84 \%$ & 90.23 & $80 \%$ \\
\hline & SD & 241.41 & 34.53 & $26 \%$ & 47.41 & $26 \%$ & 45.35 & $26 \%$ & 48.58 & $48 \%$ & 149.41 & $79 \%$ & 56.13 & $49 \%$ \\
\hline Notes & \multicolumn{14}{|c|}{$\begin{array}{l}\text { - AVE = average; } S D=\text { standard deviation; Res. }=\text { Residual } \\
\text { - Point } 1=\text { SSF Influent, Point } 1-2=\text { Phragmites, Point } 1-3=\text { Thalia; Point } 1-4=\text { Brachiaria.; } \\
\text { Point } 2-5=\text { Phragmites/Typha; Point } 3-6=\text { Thalia/Thalia; } \text { Point } 4-7=\text { Brachiaria/Cyperus } \\
\text { - Shaded cells indicates N.D. (non-detectable); - = data not available } \\
\text { - Average } \% \text { residual marked with a square around is greater than } 90 \% \text {; therefore, no further analysis was performed. }\end{array}$} \\
\hline
\end{tabular}




\begin{tabular}{|c|c|c|c|c|c|c|c|c|c|c|c|c|c|c|}
\hline \multicolumn{15}{|c|}{ Table B-18 Oil \& Grease (O\&G) Seasonal Percent Residual } \\
\hline \multirow[t]{2}{*}{ Season } & \multirow[b]{2}{*}{ Date } & \multirow{2}{*}{$\begin{array}{l}\text { O\&G Point } 1 \\
\text { Conc. (mg/L) }\end{array}$} & \multicolumn{2}{|c|}{ O\&G Point 1-2 } & \multicolumn{2}{|c|}{ O\&G Point 1-3 } & \multicolumn{2}{|c|}{ O\&G Point 1-4 } & \multicolumn{2}{|c|}{ O\&G Point 2-5 } & \multicolumn{2}{|c|}{ O\&G Point 3-6 } & \multicolumn{2}{|c|}{ O\&G Point 4-7 } \\
\hline & & & $\begin{array}{l}\text { Conc. } \\
\text { (mg/L) }\end{array}$ & $\begin{array}{c}\% \\
\text { Res. }\end{array}$ & $\begin{array}{l}\text { Conc. } \\
\text { (mg/L) }\end{array}$ & $\begin{array}{c}\% \\
\text { Res. }\end{array}$ & $\begin{array}{l}\text { Conc. } \\
\text { (mg/L) }\end{array}$ & $\begin{array}{c}\% \\
\text { Res. }\end{array}$ & $\begin{array}{l}\text { Conc. } \\
\text { (mg/L) }\end{array}$ & $\begin{array}{c}\% \\
\text { Res. }\end{array}$ & $\begin{array}{l}\text { Conc. } \\
\text { (mg/L) }\end{array}$ & $\begin{array}{c}\% \\
\text { Res. }\end{array}$ & $\begin{array}{l}\text { Conc. } \\
\text { (mg/L) }\end{array}$ & $\%$ Res. \\
\hline \multirow{11}{*}{$\begin{array}{c}\text { Wet } \\
\text { (May } \\
\text { to } \\
\text { Nov) }\end{array}$} & $11 / 29 / 06$ & 4.0 & 4.0 & $100 \%$ & 3.4 & $85 \%$ & 2.6 & $65 \%$ & 1.00 & $25 \%$ & 0.40 & $12 \%$ & 0.20 & $8 \%$ \\
\hline & $5 / 9 / 07$ & 3.4 & 9.5 & $279 \%$ & 7.9 & $232 \%$ & 12.9 & $379 \%$ & 6.90 & $73 \%$ & 8.60 & $109 \%$ & 6.70 & $52 \%$ \\
\hline & $6 / 27 / 07$ & 16.0 & 4.3 & $27 \%$ & 2.2 & $14 \%$ & 2.0 & $13 \%$ & 1.80 & $42 \%$ & 0.90 & $41 \%$ & 0.60 & $30 \%$ \\
\hline & $7 / 4 / 07$ & 0.8 & 3.8 & $475 \%$ & 1.0 & $125 \%$ & 0.0 & $0 \%$ & 1.40 & $37 \%$ & 3.90 & $390 \%$ & 5.90 & $>1000 \%$ \\
\hline & $7 / 11 / 07$ & 9.4 & 0.3 & $3 \%$ & 1.3 & $14 \%$ & 0.9 & $10 \%$ & 1.70 & $567 \%$ & 1.10 & $85 \%$ & 0.70 & $78 \%$ \\
\hline & $7 / 18 / 07$ & 5.4 & 3.1 & $57 \%$ & 7.9 & $146 \%$ & 10.5 & $194 \%$ & 0.70 & $23 \%$ & 4.40 & $56 \%$ & 1.50 & $14 \%$ \\
\hline & $7 / 25 / 07$ & 1.2 & 8.5 & $708 \%$ & 8.6 & $717 \%$ & 15.2 & $1267 \%$ & 1.00 & $12 \%$ & 1.30 & $15 \%$ & 0.20 & $1 \%$ \\
\hline & $8 / 15 / 07$ & 4.1 & 1.4 & $35 \%$ & 1.2 & $30 \%$ & 1.5 & $37 \%$ & 1.30 & $93 \%$ & 8.10 & $675 \%$ & 2.10 & $140 \%$ \\
\hline & $9 / 19 / 07$ & 5.6 & 1.0 & $18 \%$ & 0.2 & $4 \%$ & 0.9 & $16 \%$ & 0.00 & $0 \%$ & 1.50 & $750 \%$ & 1.10 & $122 \%$ \\
\hline & AVE & 5.5 & 4.0 & $189 \%$ & 3.7 & $152 \%$ & 5.2 & $220 \%$ & 1.8 & $97 \%$ & 3.4 & $237 \%$ & 2.1 & $56 \%$ \\
\hline & SD & 4.7 & 3.2 & $250 \%$ & 3.4 & $225 \%$ & 5.9 & $412 \%$ & 2.0 & $179 \%$ & 3.1 & $294 \%$ & 2.5 & $53 \%$ \\
\hline \multirow{10}{*}{$\begin{array}{l}\text { Dry } \\
\text { (Dec } \\
\text { to } \\
\text { April) }\end{array}$} & $12 / 19 / 06$ & 9.4 & 7.4 & $79 \%$ & 8.6 & $91 \%$ & 4.8 & $51 \%$ & 2.00 & $27 \%$ & 3.80 & $44 \%$ & 3.40 & $71 \%$ \\
\hline & $1 / 8 / 07$ & 0.5 & 0.4 & $80 \%$ & 0.9 & $180 \%$ & 1.1 & $220 \%$ & 0.20 & $50 \%$ & 0.40 & $44 \%$ & 0.20 & $18 \%$ \\
\hline & $1 / 17 / 07$ & 7.0 & 3.6 & $51 \%$ & 1.0 & $14 \%$ & 0.8 & $11 \%$ & 0.80 & $22 \%$ & 0.90 & $90 \%$ & 0.40 & $50 \%$ \\
\hline & $1 / 24 / 07$ & 0.6 & 0.6 & $100 \%$ & 0.6 & $100 \%$ & 1.2 & $200 \%$ & 0.50 & $83 \%$ & 0.40 & $67 \%$ & 0.50 & $42 \%$ \\
\hline & $1 / 30 / 07$ & 1.4 & 0.6 & $43 \%$ & 0.6 & $43 \%$ & 0.5 & $36 \%$ & 55.44 & $9240 \%$ & 0.80 & $133 \%$ & 1.20 & $240 \%$ \\
\hline & $2 / 7 / 07$ & 3.5 & 2.5 & $71 \%$ & 0.9 & $26 \%$ & 1.9 & $54 \%$ & 1.90 & $76 \%$ & 0.50 & $56 \%$ & 0.20 & $11 \%$ \\
\hline & $3 / 13 / 07$ & 7.1 & 6.6 & $93 \%$ & 2.3 & $32 \%$ & 2.5 & $35 \%$ & 1.40 & $21 \%$ & 1.90 & $83 \%$ & 2.50 & $100 \%$ \\
\hline & $4 / 24 / 07$ & 20.2 & 5.9 & $29 \%$ & 5.9 & $29 \%$ & 0.4 & $2 \%$ & 1.00 & $17 \%$ & 5.40 & $92 \%$ & 9.80 & $2450 \%$ \\
\hline & AVE & 6.2 & 3.5 & $68 \%$ & 2.6 & $64 \%$ & 1.7 & $76 \%$ & 7.9 & $1192 \%$ & 1.8 & $76 \%$ & 2.3 & $373 \%$ \\
\hline & SD & 6.6 & 2.9 & $25 \%$ & 3.0 & $56 \%$ & 1.5 & $85 \%$ & 19.2 & $3252 \%$ & 1.9 & $30 \%$ & 3.3 & $843 \%$ \\
\hline Notes & \multicolumn{14}{|c|}{$\begin{array}{l}\text { - AVE }=\text { average; } S D=\text { standard deviation; Res. }=\text { Residual } \\
\text { - Point } 1=S S F \text { Influent, Point } 1-2=\text { Phragmites, Point } 1-3=\text { Thalia; } \text { Point } 1-4=\text { Brachiaria.; } \\
\text { Point } 2-5=\text { Phragmites/Typha; } \text { Point } 3-6=\text { Thalia/Thalia; } \text { Point } 4-7=\text { Brachiaria/Cyperus } \\
\text { - Shaded cells indicates N.D. (non-detectable); }-=\text { data not available } \\
\text { - Average \% residual marked with a square around is greater than } 90 \% \text {; therefore, no further analysis was performed. }\end{array}$} \\
\hline
\end{tabular}




\begin{tabular}{|c|c|c|c|c|c|c|c|c|c|c|c|c|c|c|}
\hline \multicolumn{15}{|c|}{ Table B-19 Total Suspended Solids (TSS) Seasonal Percent Residual } \\
\hline \multirow[t]{2}{*}{ Season } & \multirow{2}{*}{ Date } & \multirow{2}{*}{$\begin{array}{c}\text { TSS Point } 1 \\
\text { Conc. }(\mathrm{mg} / \mathrm{L})\end{array}$} & \multicolumn{2}{|c|}{ TSS Point 1-2 } & \multicolumn{2}{|c|}{ TSS Point 1-3 } & \multicolumn{2}{|c|}{ TSS Point 1-4 } & \multicolumn{2}{|c|}{ TSS Point 2-5 } & \multicolumn{2}{|c|}{ TSS Point 3-6 } & \multicolumn{2}{|c|}{ TSS Point 4-7 } \\
\hline & & & $\begin{array}{l}\text { Conc. } \\
\text { (mg/L) }\end{array}$ & $\begin{array}{c}\% \\
\text { Res. }\end{array}$ & $\begin{array}{l}\text { Conc. } \\
\text { (mg/L) }\end{array}$ & $\begin{array}{c}\% \\
\text { Res. }\end{array}$ & $\begin{array}{l}\text { Conc. } \\
\text { (mg/L) }\end{array}$ & $\begin{array}{c}\% \\
\text { Res. }\end{array}$ & $\begin{array}{l}\text { Conc. } \\
\text { (mg/L) }\end{array}$ & $\begin{array}{c}\% \\
\text { Res. }\end{array}$ & $\begin{array}{l}\text { Conc. } \\
\text { (mg/L) }\end{array}$ & \% Res. & $\begin{array}{l}\text { Conc. } \\
\text { (mg/L) }\end{array}$ & \% Res. \\
\hline \multirow{11}{*}{$\begin{array}{c}\text { Wet } \\
\text { (May } \\
\text { to } \\
\text { Nov) }\end{array}$} & $11 / 29 / 06$ & 114 & 30 & $26 \%$ & 16 & $14 \%$ & 34 & $30 \%$ & 4.00 & $13 \%$ & 10.00 & $63 \%$ & 0.00 & $0 \%$ \\
\hline & $5 / 9 / 07$ & 152 & 4 & $3 \%$ & 0 & $0 \%$ & 0 & $0 \%$ & 0.00 & $0 \%$ & 16.00 & $16000 \%$ & 8.00 & $>1000 \%$ \\
\hline & $6 / 27 / 07$ & 100 & 0 & $0 \%$ & 0 & $0 \%$ & 40 & $0 \%$ & 0.00 & $0 \%$ & 16.00 & $16000 \%$ & 0.00 & $0 \%$ \\
\hline & $7 / 4 / 07$ & 30 & 0 & $0 \%$ & 0 & $0 \%$ & 8 & $0 \%$ & 0.00 & $0 \%$ & 12.00 & $12000 \%$ & 4.00 & $50 \%$ \\
\hline & $7 / 11 / 07$ & 25 & 6 & $24 \%$ & 4 & $16 \%$ & 6 & $24 \%$ & 1.00 & $17 \%$ & 0.00 & $0 \%$ & 0.00 & $0 \%$ \\
\hline & $7 / 18 / 07$ & 72 & 16 & $22 \%$ & 18 & $25 \%$ & 16 & $22 \%$ & 6.00 & $38 \%$ & 16.00 & $89 \%$ & 4.00 & $25 \%$ \\
\hline & $7 / 25 / 07$ & 102 & 12 & $12 \%$ & 42 & $41 \%$ & 20 & $20 \%$ & 0.00 & $0 \%$ & 0.00 & $0 \%$ & 6.00 & $30 \%$ \\
\hline & $8 / 15 / 07$ & 48 & 0 & $0 \%$ & 0 & $0 \%$ & 0 & $0 \%$ & 0.00 & $0 \%$ & 0.00 & $0 \%$ & 0.00 & $0 \%$ \\
\hline & $9 / 19 / 07$ & - & - & - & - & - & - & - & - & - & - & - & - & - \\
\hline & AVE & 80 & 9 & $11 \%$ & 10 & $12 \%$ & 16 & $12 \%$ & 1 & $8 \%$ & 9 & $5519 \%$ & 3 & $15 \%$ \\
\hline & SD & 44 & 11 & $12 \%$ & 15 & $15 \%$ & 15 & $13 \%$ & 2 & $14 \%$ & 8 & $7675 \%$ & 3 & $20 \%$ \\
\hline \multirow{10}{*}{$\begin{array}{l}\text { Dry } \\
\text { (Dec } \\
\text { to } \\
\text { April) }\end{array}$} & $12 / 19 / 06$ & 205 & 80 & $39 \%$ & 95 & $46 \%$ & 30 & $15 \%$ & 0.00 & $0 \%$ & 0.00 & $0 \%$ & 0.00 & $0 \%$ \\
\hline & $1 / 8 / 07$ & 140 & 34 & $24 \%$ & 42 & $30 \%$ & 44 & $31 \%$ & 4.00 & $12 \%$ & 6.00 & $14 \%$ & 6.00 & $14 \%$ \\
\hline & $1 / 17 / 07$ & 176 & 88 & $50 \%$ & 104 & $59 \%$ & 40 & $23 \%$ & 0.00 & $0 \%$ & 0.00 & $0 \%$ & 0.00 & $0 \%$ \\
\hline & $1 / 24 / 07$ & 136 & 4 & $3 \%$ & 20 & $15 \%$ & 4 & $3 \%$ & 4.00 & $100 \%$ & 8.00 & $40 \%$ & 4.00 & $100 \%$ \\
\hline & $1 / 30 / 07$ & 168 & 4 & $2 \%$ & 4 & $2 \%$ & 0 & $0 \%$ & 0.80 & $20 \%$ & 6.00 & $150 \%$ & 4.00 & $>1000 \%$ \\
\hline & $2 / 7 / 07$ & 160 & 6 & $4 \%$ & 4 & $3 \%$ & 16 & $10 \%$ & 8.00 & $133 \%$ & 4.00 & $100 \%$ & 4.00 & $25 \%$ \\
\hline & $3 / 13 / 07$ & 335 & 25 & $7 \%$ & 30 & $9 \%$ & 40 & $12 \%$ & 10.00 & $40 \%$ & 80.00 & $267 \%$ & 20.00 & $50 \%$ \\
\hline & $4 / 24 / 07$ & 630 & 16 & $3 \%$ & 18 & $3 \%$ & 8 & $1 \%$ & 10.00 & $63 \%$ & 82.00 & $456 \%$ & 8.00 & $100 \%$ \\
\hline & AVE & 244 & 32 & $17 \%$ & 40 & $21 \%$ & 23 & $12 \%$ & 5 & $46 \%$ & 23 & $128 \%$ & 6 & $41 \%$ \\
\hline & SD & 168 & 34 & $19 \%$ & 39 & $22 \%$ & 18 & $11 \%$ & 4 & $49 \%$ & 36 & $161 \%$ & 6 & $44 \%$ \\
\hline \multicolumn{15}{|l|}{ Notes } \\
\hline
\end{tabular}


Table B-20 Total Nitrogen (TN) Seasonal Percent Residual

\begin{tabular}{|c|c|c|c|c|c|c|c|c|c|c|c|c|c|c|}
\hline \multicolumn{15}{|c|}{ Table B-20 Total Nitrogen (TN) Seasonal Percent Residual } \\
\hline \multirow[t]{2}{*}{ Season } & \multirow[b]{2}{*}{ Date } & \multirow{2}{*}{$\begin{array}{c}\text { TN Point } 1 \\
\text { Conc. }(m g / L)\end{array}$} & \multicolumn{2}{|c|}{ TN Point 1-2 } & \multicolumn{2}{|c|}{ TN Point 1-3 } & \multicolumn{2}{|c|}{ TN Point 1-4 } & \multicolumn{2}{|c|}{ TN Point 2-5 } & \multicolumn{2}{|c|}{ TN Point 3-6 } & \multicolumn{2}{|c|}{ TN Point 4-7 } \\
\hline & & & $\begin{array}{l}\text { Conc. } \\
(\mathrm{mg} / \mathrm{L})\end{array}$ & $\begin{array}{c}\% \\
\text { Res. }\end{array}$ & $\begin{array}{l}\text { Conc. } \\
\text { (mg/L) }\end{array}$ & $\begin{array}{c}\% \\
\text { Res. }\end{array}$ & $\begin{array}{l}\text { Conc. } \\
\text { (mg/L) }\end{array}$ & $\begin{array}{c}\% \\
\text { Res. }\end{array}$ & $\begin{array}{l}\text { Conc. } \\
(\mathrm{mg} / \mathrm{L})\end{array}$ & $\begin{array}{c}\% \\
\text { Res. }\end{array}$ & $\begin{array}{l}\text { Conc. } \\
\text { (mg/L) }\end{array}$ & $\begin{array}{c}\% \\
\text { Res. }\end{array}$ & $\begin{array}{l}\text { Conc. } \\
(\mathrm{mg} / \mathrm{L})\end{array}$ & $\begin{array}{c}\% \\
\text { Res. }\end{array}$ \\
\hline \multirow{11}{*}{$\begin{array}{l}\text { Wet } \\
\text { (May } \\
\text { to } \\
\text { Nov) }\end{array}$} & $11 / 29 / 06$ & 24.46 & 16.30 & $67 \%$ & 13.59 & $56 \%$ & 16.30 & $67 \%$ & 13.59 & $83 \%$ & 10.87 & $80 \%$ & 10.87 & $67 \%$ \\
\hline & $5 / 9 / 07$ & 20.13 & 23.76 & $118 \%$ & 18.24 & $91 \%$ & 16.00 & $79 \%$ & 5.43 & $23 \%$ & 6.34 & $35 \%$ & 6.64 & $42 \%$ \\
\hline & $6 / 27 / 07$ & 21.01 & 13.18 & $63 \%$ & 12.66 & $60 \%$ & 12.96 & $62 \%$ & 2.01 & $15 \%$ & 2.73 & $22 \%$ & 2.26 & $17 \%$ \\
\hline & $7 / 4 / 07$ & 17.51 & 14.20 & $81 \%$ & 15.63 & $89 \%$ & 12.82 & $73 \%$ & 1.77 & $12 \%$ & 1.89 & $12 \%$ & 5.48 & $43 \%$ \\
\hline & $7 / 11 / 07$ & 17.14 & 13.94 & $81 \%$ & 14.11 & $82 \%$ & 12.89 & $75 \%$ & 2.03 & $15 \%$ & 2.50 & $18 \%$ & 4.20 & $33 \%$ \\
\hline & $7 / 18 / 07$ & 17.67 & 8.07 & $46 \%$ & 8.65 & $49 \%$ & 9.03 & $51 \%$ & 2.10 & $26 \%$ & 3.55 & $41 \%$ & 3.92 & $43 \%$ \\
\hline & $7 / 25 / 07$ & 25.16 & 12.02 & $48 \%$ & 11.49 & $46 \%$ & 11.47 & $46 \%$ & 4.61 & $38 \%$ & 4.72 & $41 \%$ & 6.67 & $58 \%$ \\
\hline & $8 / 15 / 07$ & 26.84 & 10.56 & $39 \%$ & 11.01 & $41 \%$ & 10.42 & $39 \%$ & 1.02 & $10 \%$ & 1.63 & $15 \%$ & 1.80 & $17 \%$ \\
\hline & $9 / 19 / 07$ & - & - & - & - & - & - & - & - & - & - & - & - & - \\
\hline & AVE & 21.24 & 14.00 & $68 \%$ & 13.17 & $64 \%$ & 12.74 & $61 \%$ & 4.07 & $28 \%$ & 4.28 & $33 \%$ & 5.23 & $40 \%$ \\
\hline & SD & 3.81 & 4.66 & $26 \%$ & 2.95 & $20 \%$ & 2.51 & $15 \%$ & 4.13 & $24 \%$ & 3.09 & $22 \%$ & 2.91 & $17 \%$ \\
\hline \multirow{10}{*}{$\begin{array}{l}\text { Dry } \\
\text { (Dec } \\
\text { to } \\
\text { April) }\end{array}$} & $12 / 19 / 06$ & 24.83 & 15.18 & $61 \%$ & 16.56 & $67 \%$ & 2.76 & $11 \%$ & 5.52 & $36 \%$ & 8.28 & $50 \%$ & 7.59 & $275 \%$ \\
\hline & $1 / 8 / 07$ & 16.56 & 11.04 & $67 \%$ & 13.80 & $83 \%$ & 8.28 & $50 \%$ & 2.76 & $25 \%$ & 8.28 & $60 \%$ & 2.76 & $33 \%$ \\
\hline & $1 / 17 / 07$ & 5.52 & 13.80 & $250 \%$ & 8.28 & $150 \%$ & 11.04 & $200 \%$ & 5.52 & $40 \%$ & 2.76 & $33 \%$ & 5.52 & $50 \%$ \\
\hline & $1 / 24 / 07$ & 2.76 & 11.04 & $400 \%$ & 10.35 & $375 \%$ & 8.28 & $300 \%$ & 5.52 & $50 \%$ & 5.52 & $53 \%$ & 2.76 & $33 \%$ \\
\hline & $1 / 30 / 07$ & 11.04 & 5.52 & $50 \%$ & 3.45 & $31 \%$ & 4.83 & $44 \%$ & 4.00 & $72 \%$ & 5.52 & $160 \%$ & 2.76 & $57 \%$ \\
\hline & $2 / 7 / 07$ & 17.94 & 5.52 & $31 \%$ & 12.42 & $69 \%$ & 9.66 & $54 \%$ & 5.52 & $100 \%$ & 5.52 & $44 \%$ & 6.90 & $71 \%$ \\
\hline & $3 / 13 / 07$ & 41.59 & 14.51 & $35 \%$ & 16.29 & $39 \%$ & 15.21 & $37 \%$ & 5.93 & $41 \%$ & 13.31 & $82 \%$ & 7.19 & $47 \%$ \\
\hline & $4 / 24 / 07$ & 25.74 & 19.70 & $77 \%$ & 19.87 & $77 \%$ & 17.86 & $69 \%$ & 7.70 & $39 \%$ & 14.27 & $72 \%$ & 11.29 & $63 \%$ \\
\hline & AVE & 18.25 & 12.04 & $121 \%$ & 12.63 & $111 \%$ & 9.74 & $96 \%$ & 5.31 & $50 \%$ & 7.93 & $69 \%$ & 5.85 & $79 \%$ \\
\hline & SD & 12.54 & 4.85 & $133 \%$ & 5.22 & $112 \%$ & 4.99 & $100 \%$ & 1.44 & $24 \%$ & 4.03 & $40 \%$ & 3.03 & $80 \%$ \\
\hline \multicolumn{15}{|l|}{ Notes } \\
\hline
\end{tabular}


Table B-21 Ammonium Seasonal Percent Residual

\begin{tabular}{|c|c|c|c|c|c|c|c|c|c|c|c|c|c|c|}
\hline & & & & Tabl & $-21 \mathrm{Am}$ & onium s & sonal $P$ & cent $R e$ & ual & & & & & \\
\hline \multirow[t]{2}{*}{ Season } & \multirow[t]{2}{*}{ Date } & \multirow[t]{2}{*}{$\begin{array}{c}\text { Ammonium Point } 1 \\
\text { Conc. (mg/L) }\end{array}$} & \multicolumn{2}{|c|}{$\begin{array}{l}\text { Ammonium } \\
\text { Point 1-2 }\end{array}$} & \multicolumn{2}{|c|}{$\begin{array}{c}\text { Ammonium } \\
\text { Point 1-3 }\end{array}$} & \multicolumn{2}{|c|}{$\begin{array}{c}\text { Ammonium } \\
\text { Point 1-4 }\end{array}$} & \multicolumn{2}{|c|}{$\begin{array}{c}\text { Ammonium } \\
\text { Point 2-5 }\end{array}$} & \multicolumn{2}{|c|}{$\begin{array}{c}\text { Ammonium } \\
\text { Point 3-6 }\end{array}$} & \multicolumn{2}{|c|}{$\begin{array}{c}\text { Ammonium } \\
\text { Point 4-7 }\end{array}$} \\
\hline & & & $\begin{array}{l}\text { Conc. } \\
(\mathrm{mg} / \mathrm{L})\end{array}$ & $\begin{array}{c}\% \\
\text { Res. }\end{array}$ & $\begin{array}{l}\text { Conc. } \\
(\mathrm{mg} / \mathrm{L})\end{array}$ & $\begin{array}{c}\% \\
\text { Res. }\end{array}$ & $\begin{array}{l}\text { Conc. } \\
\text { (mg/L) }\end{array}$ & $\begin{array}{c}\% \\
\text { Res. }\end{array}$ & $\begin{array}{l}\text { Conc. } \\
(\mathrm{mg} / \mathrm{L})\end{array}$ & $\begin{array}{l}\% \\
\text { Res. }\end{array}$ & $\begin{array}{l}\text { Conc. } \\
(\mathrm{mg} / \mathrm{L})\end{array}$ & $\begin{array}{c}\% \\
\text { Res. }\end{array}$ & $\begin{array}{l}\text { Conc. } \\
(\mathrm{mg} / \mathrm{L})\end{array}$ & $\begin{array}{c}\% \\
\text { Res. }\end{array}$ \\
\hline \multirow{11}{*}{$\begin{array}{c}\text { Wet } \\
\text { (May } \\
\text { to } \\
\text { Nov) }\end{array}$} & $11 / 29 / 06$ & 1.08 & 2.85 & $264 \%$ & 3.21 & $297 \%$ & 2.85 & $264 \%$ & 0.15 & $5 \%$ & 0.09 & $3 \%$ & 0.11 & $4 \%$ \\
\hline & $5 / 9 / 07$ & 2.25 & 18.69 & $831 \%$ & 14.16 & $629 \%$ & 12.39 & $551 \%$ & 1.04 & $6 \%$ & 0.19 & $1 \%$ & 0.07 & $1 \%$ \\
\hline & $6 / 27 / 07$ & 7.40 & 8.66 & $117 \%$ & 9.13 & $123 \%$ & 9.13 & $123 \%$ & 0.12 & $1 \%$ & 0.13 & $1 \%$ & 0.16 & $2 \%$ \\
\hline & $7 / 4 / 07$ & 14.67 & 12.52 & $85 \%$ & 11.88 & $81 \%$ & 11.27 & $77 \%$ & 0.00 & $0 \%$ & 0.45 & $4 \%$ & 0.56 & $5 \%$ \\
\hline & $7 / 11 / 07$ & 9.51 & 3.42 & $36 \%$ & 9.22 & $97 \%$ & 0.84 & $9 \%$ & 0.19 & $6 \%$ & 0.67 & $7 \%$ & 0.14 & $17 \%$ \\
\hline & $7 / 18 / 07$ & 12.69 & 5.35 & $42 \%$ & 7.37 & $58 \%$ & 8.64 & $68 \%$ & 0.16 & $3 \%$ & 1.64 & $22 \%$ & 0.14 & $2 \%$ \\
\hline & $7 / 25 / 07$ & 21.85 & 11.16 & $51 \%$ & 10.14 & $46 \%$ & 11.16 & $51 \%$ & 3.76 & $34 \%$ & 428.00 & $4221 \%$ & 5.70 & $51 \%$ \\
\hline & $8 / 15 / 07$ & 15.80 & 10.39 & $66 \%$ & 8.73 & $55 \%$ & 8.73 & $55 \%$ & 0.35 & $3 \%$ & 0.90 & $10 \%$ & 1.19 & $14 \%$ \\
\hline & $9 / 19 / 07$ & 15.84 & 7.89 & $50 \%$ & 7.34 & $46 \%$ & 7.89 & $50 \%$ & 0.00 & $0 \%$ & 0.00 & $0 \%$ & 0.00 & $0 \%$ \\
\hline & AVE & 11.23 & 8.99 & $171 \%$ & 9.02 & $159 \%$ & 8.10 & $139 \%$ & 0.64 & $6 \%$ & 48.01 & $474 \%$ & 0.90 & $10 \%$ \\
\hline & SD & 6.80 & 4.96 & $257 \%$ & 3.07 & $193 \%$ & 3.87 & $171 \%$ & 1.21 & $10 \%$ & 142.50 & $1405 \%$ & 1.84 & $16 \%$ \\
\hline \multirow{10}{*}{$\begin{array}{c}\text { Dry } \\
\text { (Dec to } \\
\text { April) }\end{array}$} & $12 / 19 / 06$ & 5.16 & 4.60 & $89 \%$ & 8.14 & $158 \%$ & 5.56 & $108 \%$ & 0.13 & $3 \%$ & 0.09 & $1 \%$ & 0.57 & $10 \%$ \\
\hline & $1 / 8 / 07$ & 0.16 & 5.56 & $3475 \%$ & 7.06 & $4413 \%$ & 5.79 & $3619 \%$ & 0.14 & $3 \%$ & 0.07 & $1 \%$ & 0.04 & $1 \%$ \\
\hline & $1 / 17 / 07$ & 0.17 & 1.75 & $1029 \%$ & 1.22 & $718 \%$ & 4.04 & $2376 \%$ & 0.17 & $10 \%$ & 0.47 & $39 \%$ & 0.67 & $17 \%$ \\
\hline & $1 / 24 / 07$ & 0.19 & 3.24 & $1705 \%$ & 4.47 & $2353 \%$ & 4.12 & $2168 \%$ & 0.17 & $5 \%$ & 0.30 & $7 \%$ & 0.28 & $7 \%$ \\
\hline & $1 / 30 / 07$ & 0.67 & 6.42 & $958 \%$ & 7.59 & $1133 \%$ & 5.90 & $881 \%$ & 2.76 & $43 \%$ & 0.34 & $4 \%$ & 0.23 & $4 \%$ \\
\hline & $2 / 7 / 07$ & 2.71 & 11.51 & $425 \%$ & 13.86 & $511 \%$ & 12.40 & $458 \%$ & 2.71 & $24 \%$ & 1.50 & $11 \%$ & 0.40 & $3 \%$ \\
\hline & $3 / 13 / 07$ & - & - & - & - & - & - & - & - & - & - & - & - & - \\
\hline & $4 / 24 / 07$ & 6.56 & 13.69 & $209 \%$ & 13.69 & $209 \%$ & 12.39 & $189 \%$ & 0.29 & $2 \%$ & 1.97 & $14 \%$ & 0.35 & $3 \%$ \\
\hline & AVE & 2.23 & 6.68 & $1127 \%$ & 8.00 & $1356 \%$ & 7.17 & $1400 \%$ & 0.91 & $13 \%$ & 0.68 & $11 \%$ & 0.36 & $6 \%$ \\
\hline & SD & 2.67 & 4.36 & $1176 \%$ & 4.59 & $1542 \%$ & 3.65 & $1339 \%$ & 1.25 & $15 \%$ & 0.75 & $13 \%$ & 0.21 & $5 \%$ \\
\hline Notes & \multicolumn{14}{|c|}{$\begin{array}{l}\text { - AVE = average; SD = standard deviation; Res. }=\text { Residual } \\
\text { - Point } 1=S S F \text { Influent, Point } 1-2=\text { Phragmites, Point } 1-3=\text { Thalia; Point } 1-4=\text { Brachiaria.; } \\
\text { Point } 2-5=\text { Phragmites/Typha; Point } 3-6=\text { Thalia/Thalia; } \text { Point } 4-7=\text { Brachiaria/Cyperus } \\
\text { - Shaded cells indicates N.D. (non-detectable); }-=\text { data not available } \\
\text { - Average } \% \text { residual marked with a square around is greater than } 90 \% \text {; therefore, no further analysis was performed. }\end{array}$} \\
\hline
\end{tabular}




\begin{tabular}{|c|c|c|c|c|c|c|c|c|c|c|c|c|c|c|c|c|c|c|c|c|c|c|c|c|c|c|c|c|c|}
\hline \multicolumn{30}{|c|}{ Table B-22 Summary of Seasonal Average Performance - SSF CW } \\
\hline \multirow[t]{4}{*}{ Season } & \multirow[t]{4}{*}{ Parameter } & \multirow{2}{*}{\multicolumn{4}{|c|}{$\begin{array}{c}\text { Point } 1 \\
\text { Concentration }\end{array}$}} & \multicolumn{8}{|c|}{ Point 1-2 } & \multicolumn{8}{|c|}{ Point 1-3 } & \multicolumn{8}{|c|}{ Point 1-4 } \\
\hline & & & & & & \multicolumn{4}{|c|}{ Concentration } & \multicolumn{4}{|c|}{ \% Residual } & \multicolumn{4}{|c|}{ Concentration } & \multicolumn{4}{|c|}{$\%$ Residual } & \multicolumn{4}{|c|}{ Concentration } & \multicolumn{4}{|c|}{$\%$ Residual } \\
\hline & & \multirow[t]{2}{*}{ AVE } & \multirow[t]{2}{*}{ SD } & \multicolumn{2}{|c|}{ 95\% C.I. } & \multirow[t]{2}{*}{ AVE } & \multirow[t]{2}{*}{ SD } & \multicolumn{2}{|c|}{ 95\% C.I. } & \multirow[t]{2}{*}{ AVE } & \multirow[t]{2}{*}{ SD } & \multicolumn{2}{|c|}{ 95\% C.I. } & \multirow[t]{2}{*}{ AVE } & \multirow[t]{2}{*}{ SD } & \multicolumn{2}{|c|}{ 95\% C.I. } & AVE & SD & $95 \%$ & & AVE & SD & & & AVE & SD & $95 \%$ & C.I. \\
\hline & & & & Min & Max & & & Min & Max & & & Min & Max & & & Min & Max & & & Min & Max & & & Min & Max & & & Min & Max \\
\hline $\begin{array}{l}\text { Wet } \\
\text { (May }\end{array}$ & $\begin{array}{c}\text { FC. }(\mathrm{NMP} / 100 \\
\mathrm{mL})\end{array}$ & 112,489 & 113,039 & 25,600 & 199,378 & 12,764 & 16,701 & -73 & 25,602 & $18 \%$ & $19 \%$ & $4 \%$ & $32 \%$ & 32,333 & 23,796 & 14,042 & 50,625 & $80 \%$ & $95 \%$ & $7 \%$ & $153 \%$ & 17,204 & 28,561 & 4,750 & 39,158 & $20 \%$ & $25 \%$ & $1 \%$ & $39 \%$ \\
\hline Nov) & BOD $(\mathrm{mg} / \mathrm{L})$ & 53.8 & 16.3 & 41.3 & 66.3 & 39.6 & 20.9 & 23.5 & 55.6 & $80 \%$ & $62 \%$ & $32 \%$ & $128 \%$ & 33.3 & 10.0 & 25.6 & 41.0 & $64 \%$ & $17 \%$ & $51 \%$ & $77 \%$ & 33.5 & 8.2 & 27.2 & 39.7 & $65 \%$ & $19 \%$ & $51 \%$ & $80 \%$ \\
\hline & $\operatorname{COD}(m \mathrm{~g} / \mathrm{L})$ & 185.2 & 81.2 & 117.3 & 253.2 & 86.2 & 53.1 & 41.8 & 130.6 & $53 \%$ & $31 \%$ & $27 \%$ & $78 \%$ & 106.3 & 55.4 & 60.0 & 152.6 & $62 \%$ & $27 \%$ & $40 \%$ & $85 \%$ & 73.5 & 37.0 & 42.5 & 104.5 & $47 \%$ & $28 \%$ & $23 \%$ & $70 \%$ \\
\hline & $O \& G(m g / L)$ & 5.5 & 4.7 & 1.9 & 9.1 & 4.0 & 3.2 & 1.5 & 6.4 & $189 \%$ & $250 \%$ & $-3 \%$ & $381 \%$ & 3.7 & 3.4 & 1.1 & 6.4 & $152 \%$ & $225 \%$ & $-21 \%$ & $325 \%$ & 5.2 & 5.9 & 0.6 & 9.7 & $220 \%$ & $412 \%$ & $96 \%$ & $537 \%$ \\
\hline & TSS (mg/L) & 80.4 & 44.5 & 43.2 & 117.5 & 8.5 & 10.5 & -0.3 & 17.3 & $11 \%$ & $12 \%$ & $1 \%$ & $21 \%$ & 10.0 & 14.9 & -2.5 & 22.5 & $12 \%$ & $15 \%$ & $-1 \%$ & $25 \%$ & 15.5 & 15.1 & 2.9 & 28.1 & $20 \%$ & $14 \%$ & $9 \%$ & $32 \%$ \\
\hline & $\mathrm{TN}(\mathrm{mg} / \mathrm{L})$ & 21.2 & 3.8 & 18.1 & 24.4 & 14.0 & 4.7 & 10.1 & 17.9 & $68 \%$ & $26 \%$ & $46 \%$ & $89 \%$ & 13.2 & 3.0 & 10.7 & 15.6 & $64 \%$ & $20 \%$ & $47 \%$ & $81 \%$ & 12.7 & 2.5 & 10.6 & 14.8 & $61 \%$ & $15 \%$ & $49 \%$ & $74 \%$ \\
\hline & TP (mg/L) & 2.1 & 1.5 & 0.9 & 3.3 & 3.2 & 0.9 & 2.5 & 3.9 & $288 \%$ & $265 \%$ & $84 \%$ & $492 \%$ & 2.9 & 1.3 & 1.9 & 3.9 & $214 \%$ & $207 \%$ & $56 \%$ & $373 \%$ & 2.9 & 1.0 & 2.1 & 3.7 & $264 \%$ & $266 \%$ & $60 \%$ & $468 \%$ \\
\hline $\begin{array}{l}\text { Dry } \\
\text { (Dec to }\end{array}$ & $\underset{\mathrm{mL})}{\mathrm{FC}(\mathrm{NMP} / \mathbf{1 0 0}}$ & 274,857 & 584,829 & 266,020 & 815,734 & 9,749 & 8,173 & 2,190 & 17,307 & $12 \%$ & $12 \%$ & $1 \%$ & $24 \%$ & 20,724 & 15,025 & 6,828 & 34,620 & $40 \%$ & $43 \%$ & $0 \%$ & $80 \%$ & 15,407 & 18,430 & 1,638 & 32,452 & $15 \%$ & $20 \%$ & $-3 \%$ & $33 \%$ \\
\hline April) & BOD (mg/L) & 81.3 & 30.8 & 55.5 & 107.0 & 62.8 & 13.6 & 51.5 & 74.1 & $82 \%$ & $22 \%$ & $63 \%$ & $101 \%$ & 76.0 & 40.2 & 42.4 & 109.6 & $94 \%$ & $31 \%$ & $68 \%$ & $120 \%$ & 48.9 & 14.2 & 37.1 & 60.8 & $64 \%$ & $21 \%$ & $46 \%$ & $81 \%$ \\
\hline & $\operatorname{COD}(m g / L)$ & 354.1 & 241.4 & 152.3 & 556.0 & 147.1 & 34.5 & 118.3 & 176.0 & $53 \%$ & $26 \%$ & $32 \%$ & $75 \%$ & 176.8 & 47.4 & 137.2 & 216.4 & $62 \%$ & $26 \%$ & $40 \%$ & $84 \%$ & 121.8 & 45.4 & 83.9 & 159.7 & $44 \%$ & $26 \%$ & $22 \%$ & $66 \%$ \\
\hline & O\&G (mg/L) & 6.2 & 6.6 & 0.7 & 11.7 & 3.5 & 2.9 & 1.0 & 5.9 & $68 \%$ & $25 \%$ & $48 \%$ & $89 \%$ & 2.6 & 3.0 & 0.1 & 5.1 & $64 \%$ & $56 \%$ & $18 \%$ & $111 \%$ & 1.7 & 1.5 & 0.4 & 2.9 & $76 \%$ & $85 \%$ & $5 \%$ & $147 \%$ \\
\hline & $\operatorname{TSS}(\mathrm{mg} / \mathbf{L})$ & 243.8 & 168.5 & 102.9 & 384.6 & 32.1 & 33.8 & 3.9 & 60.4 & $17 \%$ & $19 \%$ & $1 \%$ & $32 \%$ & 39.6 & 39.1 & 6.9 & 72.3 & $21 \%$ & $22 \%$ & $2 \%$ & $39 \%$ & 22.8 & 17.9 & 7.8 & 37.7 & $12 \%$ & $11 \%$ & $3 \%$ & $21 \%$ \\
\hline & $\mathrm{TN}(\mathrm{mg} / \mathrm{L})$ & 18.2 & 12.5 & 7.8 & 28.7 & 12.0 & 4.9 & 8.0 & 16.1 & $121 \%$ & $133 \%$ & $10 \%$ & $232 \%$ & 12.6 & 5.2 & 8.3 & 17.0 & $111 \%$ & $112 \%$ & $18 \%$ & $205 \%$ & 9.7 & 5.0 & 5.6 & 13.9 & $96 \%$ & $100 \%$ & $12 \%$ & $179 \%$ \\
\hline & TP (mg/L) & 1.5 & 0.8 & 0.8 & 2.1 & 2.6 & 1.1 & 1.7 & 3.5 & $198 \%$ & $112 \%$ & $104 \%$ & $291 \%$ & 2.4 & 1.1 & 1.5 & 3.3 & $178 \%$ & $94 \%$ & $100 \%$ & $257 \%$ & 2.4 & 1.1 & 1.5 & 3.4 & $179 \%$ & $96 \%$ & $99 \%$ & $260 \%$ \\
\hline
\end{tabular}




\begin{tabular}{|c|c|c|c|c|c|c|c|c|c|c|c|c|c|c|c|c|c|c|c|c|c|c|c|c|c|}
\hline \multicolumn{26}{|c|}{ Table B-23 Summary of Seasonal Average Performance - SF CW } \\
\hline \multirow[t]{4}{*}{ Season } & \multirow[t]{4}{*}{ Parameter } & \multicolumn{8}{|c|}{ Point 2-5 } & \multicolumn{8}{|c|}{ Point 3-6 } & \multicolumn{8}{|c|}{ Point 4-7 } \\
\hline & & \multicolumn{4}{|c|}{ Concentration } & \multicolumn{4}{|c|}{ \% Residual } & \multicolumn{4}{|c|}{ Concentration } & \multicolumn{4}{|c|}{ \% Residual } & \multicolumn{4}{|c|}{ Concentration } & \multicolumn{4}{|c|}{ \% Residual } \\
\hline & & \multirow[t]{2}{*}{ AVE } & \multirow[t]{2}{*}{ SD } & \multicolumn{2}{|c|}{ 95\% C.I. } & \multirow[t]{2}{*}{ AVE } & \multirow[t]{2}{*}{ SD } & \multicolumn{2}{|c|}{ 95\% C.I. } & \multirow[t]{2}{*}{ AVE } & \multirow[t]{2}{*}{ SD } & \multicolumn{2}{|c|}{ 95\% C.I. } & \multirow[t]{2}{*}{ AVE } & \multirow[t]{2}{*}{ SD } & \multicolumn{2}{|c|}{ 95\% C.I. } & \multirow[t]{2}{*}{ AVE } & \multirow[t]{2}{*}{ SD } & \multicolumn{2}{|c|}{ 95\% C.I. } & AVE & SD & $95 \%$ & \\
\hline & & & & Min & Max & & & Min & Max & & & Min & Max & & & Min & Max & & & Min & Max & & & Min & Max \\
\hline Wet & Fecal Colif. (NMP/100 mL) & 448 & 353 & 176 & 719 & $21 \%$ & $27 \%$ & $1 \%$ & $41 \%$ & 4,597 & 5,529 & 347 & 8,847 & $44 \%$ & $104 \%$ & $-36 \%$ & $123 \%$ & 1,646 & 2,042 & 76 & 3,215 & $269 \%$ & $684 \%$ & $-257 \%$ & $795 \%$ \\
\hline $\begin{array}{c}\text { (May to } \\
\text { Nov) }\end{array}$ & BOD $(\mathrm{mg} / \mathrm{L})$ & 20.2 & 8.1 & 14.0 & 26.4 & $60 \%$ & $23 \%$ & $43 \%$ & $78 \%$ & 21.7 & 10.7 & 13.5 & 29.9 & $64 \%$ & $19 \%$ & $49 \%$ & $78 \%$ & 18.5 & 11.6 & 9.6 & 27.4 & $53 \%$ & $26 \%$ & $33 \%$ & $73 \%$ \\
\hline & $\operatorname{COD}(\mathrm{mg} / \mathrm{L})$ & 74.3 & 46.2 & 35.7 & 112.9 & $124 \%$ & $101 \%$ & $40 \%$ & $209 \%$ & 115.4 & 124.4 & 11.5 & 219.4 & $176 \%$ & $278 \%$ & $-56 \%$ & $409 \%$ & 59.5 & 34.7 & 30.5 & 88.5 & $105 \%$ & $115 \%$ & $9 \%$ & $202 \%$ \\
\hline & Oil \& Grease (mg/L) & 1.8 & 2.0 & 0.2 & 3.3 & $97 \%$ & $179 \%$ & $-41 \%$ & $234 \%$ & 3.4 & 3.1 & 0.9 & 5.8 & $237 \%$ & $294 \%$ & $11 \%$ & $463 \%$ & 2.1 & 2.5 & 0.2 & 4.0 & $56 \%$ & $53 \%$ & $15 \%$ & $96 \%$ \\
\hline & $\operatorname{TSS}(\mathrm{mg} / \mathrm{L})$ & 1.4 & 2.3 & -0.6 & 3.3 & $8 \%$ & $14 \%$ & $-3 \%$ & $20 \%$ & 8.8 & 7.6 & 2.4 & 15.1 & $5519 \%$ & $7675 \%$ & $-898 \%$ & $11935 \%$ & 2.8 & 3.2 & 0.1 & 5.4 & $1013 \%$ & $2823 \%$ & $-1347 \%$ & $3373 \%$ \\
\hline & $\mathrm{TN}(\mathrm{mg} / \mathrm{L})$ & 4.1 & 4.1 & 0.6 & 7.5 & $28 \%$ & $24 \%$ & $8 \%$ & $48 \%$ & 4.3 & 3.1 & 1.7 & 6.9 & $33 \%$ & $22 \%$ & $14 \%$ & $52 \%$ & 5.2 & 2.9 & 2.8 & 7.7 & $40 \%$ & $17 \%$ & $25 \%$ & $55 \%$ \\
\hline & TP (mg/L) & 1.4 & 1.0 & 0.6 & 2.2 & $43 \%$ & $34 \%$ & $17 \%$ & $69 \%$ & 1.4 & 0.9 & 0.8 & 2.1 & $95 \%$ & $156 \%$ & $-25 \%$ & $215 \%$ & 1.2 & 0.8 & 0.6 & 1.8 & $45 \%$ & $40 \%$ & $15 \%$ & $76 \%$ \\
\hline Dry & Fecal Colif., (NMP/100 mL) & 338 & 322 & 41 & 635 & $6 \%$ & $4 \%$ & $2 \%$ & $10 \%$ & 1,833 & 2,214 & -215 & 3,880 & $58 \%$ & $126 \%$ & $-58 \%$ & $175 \%$ & 2,260 & 2,738 & -273 & 4,793 & $335 \%$ & $823 \%$ & $-426 \%$ & $1096 \%$ \\
\hline April) & BOD (mg/L) & 79.2 & 178.2 & -69.8 & 228.2 & $146 \%$ & $339 \%$ & $-138 \%$ & $430 \%$ & 23.4 & 13.6 & 12.0 & 34.7 & $36 \%$ & $23 \%$ & $18 \%$ & $55 \%$ & 28.9 & 22.4 & 10.2 & 47.6 & $66 \%$ & $55 \%$ & $19 \%$ & $112 \%$ \\
\hline & $\operatorname{COD}(\mathrm{mg} / \mathrm{L})$ & 71.7 & 48.6 & 31.1 & 112.3 & $55 \%$ & $48 \%$ & $15 \%$ & $96 \%$ & 143.3 & 149.4 & 18.4 & 268.2 & $84 \%$ & $79 \%$ & $18 \%$ & $149 \%$ & 90.2 & 56.1 & 43.3 & 137.2 & $80 \%$ & $49 \%$ & $39 \%$ & $121 \%$ \\
\hline & Oil \& Grease $(\mathrm{mg} / \mathrm{L})$ & 7.9 & 19.2 & -8.2 & 24.0 & $1192 \%$ & $3252 \%$ & $-1527 \%$ & $3911 \%$ & 1.8 & 1.9 & 0.2 & 3.3 & $76 \%$ & $30 \%$ & $51 \%$ & $101 \%$ & 2.3 & 3.3 & -0.5 & 5.0 & $373 \%$ & $843 \%$ & $-332 \%$ & $1077 \%$ \\
\hline & $\operatorname{TSS}(\mathbf{m g} / \mathbf{L})$ & 4.6 & 4.3 & 1.0 & 8.2 & $46 \%$ & $49 \%$ & $5 \%$ & $87 \%$ & 23.3 & 35.8 & -6.6 & 53.1 & $128 \%$ & $161 \%$ & $-6 \%$ & $263 \%$ & 5.8 & 6.4 & 0.4 & 11.1 & $536 \%$ & $1400 \%$ & $-635 \%$ & $1707 \%$ \\
\hline & $\mathrm{TN}(\mathrm{mg} / \mathrm{L})$ & 5.3 & 1.4 & 4.1 & 6.5 & $50 \%$ & $24 \%$ & $30 \%$ & $71 \%$ & 7.9 & 4.0 & 4.6 & 11.3 & $69 \%$ & $40 \%$ & $36 \%$ & $102 \%$ & 5.8 & 3.0 & 3.3 & 8.4 & $79 \%$ & $80 \%$ & $12 \%$ & $146 \%$ \\
\hline & TP (mg/L) & 2.4 & 1.7 & 1.0 & 3.8 & $89 \%$ & $65 \%$ & $34 \%$ & $144 \%$ & 1.8 & 1.0 & 0.9 & 2.6 & $79 \%$ & $35 \%$ & $50 \%$ & $109 \%$ & 2.7 & 1.0 & 1.8 & 3.5 & $114 \%$ & $43 \%$ & $78 \%$ & $150 \%$ \\
\hline
\end{tabular}




\begin{tabular}{|c|c|c|c|c|c|c|c|c|c|c|c|c|c|c|c|c|c|c|c|c|c|c|c|c|c|}
\hline \multicolumn{26}{|c|}{ Table B-24 Summary of Seasonal Average Performance - CW System (SF +SSF) } \\
\hline \multirow[t]{4}{*}{ Season } & \multirow[t]{4}{*}{ Parameter } & \multicolumn{8}{|c|}{ Point 1-2-5 } & \multicolumn{8}{|c|}{ Point 1-3-6 } & \multicolumn{8}{|c|}{ Point 1-4-7 } \\
\hline & & \multicolumn{4}{|c|}{ Concentration } & \multicolumn{4}{|c|}{$\%$ Residual } & \multicolumn{4}{|c|}{ Concentration } & \multicolumn{4}{|c|}{$\%$ Residual } & \multicolumn{4}{|c|}{ Concentration } & \multicolumn{4}{|c|}{$\%$ Residual } \\
\hline & & \multirow[t]{2}{*}{ AVE } & \multirow[t]{2}{*}{ SD } & \multicolumn{2}{|c|}{ 95\% C.I. } & \multirow[t]{2}{*}{ AVE } & \multirow[t]{2}{*}{ SD } & \multicolumn{2}{|c|}{ 95\% C.I. } & \multirow[t]{2}{*}{ AVE } & \multirow[t]{2}{*}{ SD } & \multicolumn{2}{|c|}{ 95\% C.I. } & \multirow[t]{2}{*}{ AVE } & \multirow[t]{2}{*}{ SD } & \multicolumn{2}{|c|}{ 95\% C.I. } & \multirow[t]{2}{*}{ AVE } & \multirow[t]{2}{*}{ SD } & \multicolumn{2}{|c|}{ 95\% C.I. } & AVE & SD & & C.I. \\
\hline & & & & Min & Max & & & Min & Max & & & Min & Max & & & Min & Max & & & Min & Max & & & Min & Max \\
\hline Wet & Fecal Colif. (NMP/100 mL) & 448 & 353 & 176 & 719 & $2 \%$ & $2 \%$ & $0 \%$ & $3 \%$ & 4,597 & 5,529 & 347 & 8,847 & $77 \%$ & $221 \%$ & $-93 \%$ & $247 \%$ & 1,646 & 2,042 & 76 & 3,215 & $25 \%$ & $69 \%$ & $-28 \%$ & $78 \%$ \\
\hline Nov) & BOD (mg/L) & 20.2 & 8.1 & 14.0 & 26.4 & $39 \%$ & $13 \%$ & $29 \%$ & $49 \%$ & 21.7 & 10.7 & 13.5 & 29.9 & $42 \%$ & $20 \%$ & $27 \%$ & $57 \%$ & 18.5 & 11.6 & 9.6 & 27.4 & $36 \%$ & $25 \%$ & $17 \%$ & $55 \%$ \\
\hline & $\operatorname{COD}(m g / L)$ & 74.3 & 46.2 & 35.7 & 112.9 & $54 \%$ & $56 \%$ & $7 \%$ & $101 \%$ & 115.4 & 124.4 & 11.5 & 219.4 & $73 \%$ & $76 \%$ & $10 \%$ & $137 \%$ & 59.5 & 34.7 & 30.5 & 88.5 & $38 \%$ & $24 \%$ & $18 \%$ & $59 \%$ \\
\hline & Oil \& Grease (mg/L) & 1.8 & 2.0 & 0.2 & 3.3 & $62 \%$ & $76 \%$ & $4 \%$ & $121 \%$ & 3.4 & 3.1 & 0.9 & 5.8 & $132 \%$ & $160 \%$ & $9 \%$ & $255 \%$ & 2.1 & 2.5 & 0.2 & 4.0 & $41 \%$ & $65 \%$ & $-9 \%$ & $91 \%$ \\
\hline & TSS (mg/L) & 1.4 & 2.3 & -0.6 & 3.3 & $2 \%$ & $3 \%$ & $-1 \%$ & $5 \%$ & 8.8 & 7.6 & 2.4 & 15.1 & $12 \%$ & $14 \%$ & $1 \%$ & $24 \%$ & 2.8 & 3.2 & 0.1 & 5.4 & $4 \%$ & $5 \%$ & $0 \%$ & $8 \%$ \\
\hline & $\mathrm{TN}(\mathrm{mg} / \mathrm{L})$ & 4.1 & 4.1 & 0.6 & 7.5 & $19 \%$ & $16 \%$ & $5 \%$ & $32 \%$ & 4.3 & 3.1 & 1.7 & 6.9 & $20 \%$ & $12 \%$ & $9 \%$ & $30 \%$ & 5.2 & 2.9 & 2.8 & 7.7 & $25 \%$ & $12 \%$ & $15 \%$ & $35 \%$ \\
\hline & $\mathrm{TP}(\mathrm{mg} / \mathrm{L})$ & 1.4 & 1.0 & 0.6 & 2.2 & $156 \%$ & $172 \%$ & $24 \%$ & $289 \%$ & 1.4 & 0.9 & 0.8 & 2.1 & $162 \%$ & $194 \%$ & $13 \%$ & $311 \%$ & 1.2 & 0.8 & 0.6 & 1.8 & $127 \%$ & $137 \%$ & $22 \%$ & $232 \%$ \\
\hline Dry & Fecal Colif., (NMP/100 mL) & 338 & 322 & 41 & 635 & $0 \%$ & $1 \%$ & $0 \%$ & $1 \%$ & 1,833 & 2,214 & -215 & 3,880 & $2 \%$ & $3 \%$ & $0 \%$ & $5 \%$ & 2,260 & 2,738 & -273 & 4,793 & $3 \%$ & $3 \%$ & $0 \%$ & $6 \%$ \\
\hline April) & BOD $(\mathrm{mg} / \mathrm{L})$ & 79.2 & 178.2 & -69.8 & 228.2 & $98 \%$ & $213 \%$ & $-80 \%$ & $277 \%$ & 23.4 & 13.6 & 12.0 & 34.7 & $33 \%$ & $22 \%$ & $14 \%$ & $51 \%$ & 28.9 & 22.4 & 10.2 & 47.6 & $39 \%$ & $29 \%$ & $15 \%$ & $64 \%$ \\
\hline & $\operatorname{COD}(\mathrm{mg} / \mathrm{L})$ & 71.7 & 48.6 & 31.1 & 112.3 & $25 \%$ & $22 \%$ & $6 \%$ & $43 \%$ & 143.3 & 149.4 & 18.4 & 268.2 & $38 \%$ & $28 \%$ & $15 \%$ & $62 \%$ & 90.2 & 56.1 & 43.3 & 137.2 & $31 \%$ & $25 \%$ & $10 \%$ & $52 \%$ \\
\hline & Oil \& Grease (mg/L) & 7.9 & 19.2 & -8.2 & 24.0 & $524 \%$ & $1388 \%$ & $-636 \%$ & $1685 \%$ & 1.8 & 1.9 & 0.2 & 3.3 & $41 \%$ & $25 \%$ & $20 \%$ & $61 \%$ & 2.3 & 3.3 & -0.5 & 5.0 & $43 \%$ & $30 \%$ & $17 \%$ & $68 \%$ \\
\hline & TSS (mg/L) & 4.6 & 4.3 & 1.0 & 8.2 & $2 \%$ & $2 \%$ & $0 \%$ & $3 \%$ & 23.3 & 35.8 & -6.6 & 53.1 & $7 \%$ & $8 \%$ & $0 \%$ & $13 \%$ & 5.8 & 6.4 & 0.4 & 11.1 & $2 \%$ & $2 \%$ & $1 \%$ & $4 \%$ \\
\hline & $\mathrm{TN}(\mathrm{mg} / \mathrm{L})$ & 5.3 & 1.4 & 4.1 & 6.5 & $56 \%$ & $64 \%$ & $3 \%$ & $110 \%$ & 7.9 & 4.0 & 4.6 & 11.3 & $63 \%$ & $56 \%$ & $16 \%$ & $110 \%$ & 5.8 & 3.0 & 3.3 & 8.4 & $46 \%$ & $34 \%$ & $18 \%$ & $75 \%$ \\
\hline & TP (mg/L) & 2.4 & 1.7 & 1.0 & 3.8 & $180 \%$ & $142 \%$ & $61 \%$ & $299 \%$ & 1.8 & 1.0 & 0.9 & 2.6 & $124 \%$ & $73 \%$ & $63 \%$ & $184 \%$ & 2.7 & 1.0 & 1.8 & 3.5 & $203 \%$ & $107 \%$ & $114 \%$ & $292 \%$ \\
\hline
\end{tabular}




\begin{tabular}{|c|c|c|c|c|c|c|c|c|c|}
\hline \multicolumn{10}{|c|}{ Table B-25 Field Parameter: Temperature (degree Celsius) } \\
\hline Date & $\begin{array}{c}\text { Point } \\
1\end{array}$ & $\begin{array}{c}\text { Point } \\
1-2\end{array}$ & $\begin{array}{l}\text { OW1- } \\
2-5 a_{B}\end{array}$ & $\begin{array}{l}\text { OW1- } \\
2-5 a_{M}\end{array}$ & $\begin{array}{l}\text { OW1- } \\
2-5 a_{s}\end{array}$ & $\begin{array}{l}\text { OW1- } \\
2-5 b_{B}\end{array}$ & $\begin{array}{l}\text { OW1- } \\
2-5 b_{M}\end{array}$ & $\begin{array}{l}\text { OW1- } \\
2-5 b_{S}\end{array}$ & $\begin{array}{c}\text { Point } \\
2-5\end{array}$ \\
\hline $10 / 19 / 2006$ & 0.6 & 0.7 & 0.6 & 0.6 & 0.6 & 0.3 & 0.3 & 0.3 & 0.3 \\
\hline $11 / 6 / 2006$ & 0.7 & 0.6 & 0.4 & 0.5 & 0.4 & 0.4 & 0.3 & 0.4 & 0.3 \\
\hline $12 / 19 / 2006$ & 0.8 & 0.8 & 0.3 & 0.6 & 0.5 & 0.7 & 0.6 & 0.5 & 0.5 \\
\hline $1 / 8 / 2007$ & 0.8 & 0.9 & 0.8 & 0.8 & 0.8 & 0.7 & 0.7 & 0.7 & 0.8 \\
\hline $1 / 17 / 2007$ & 0.7 & 0.7 & 0.8 & 0.8 & 0.7 & 0.7 & 0.7 & 0.7 & 0.7 \\
\hline $1 / 24 / 2007$ & 0.7 & 0.7 & 0.7 & 0.7 & 0.7 & 0.7 & 0.7 & 0.7 & 0.8 \\
\hline $1 / 30 / 2007$ & 0.7 & 0.8 & 0.8 & 0.8 & 0.8 & 0.7 & 0.7 & 0.7 & 0.8 \\
\hline $2 / 7 / 2007$ & 0.8 & 0.9 & 0.8 & 0.8 & 0.8 & 0.7 & 0.7 & 0.8 & 0.9 \\
\hline $3 / 28 / 2007$ & 1.9 & 2.9 & 2.3 & 2.2 & 2.3 & 2.2 & 2.1 & 2.1 & 2.6 \\
\hline $4 / 24 / 2007$ & 1.7 & 2.2 & 2.7 & 2.3 & 2.3 & 2.5 & 2.3 & 2.3 & 2.2 \\
\hline $5 / 9 / 2007$ & 1.4 & 1.7 & 2.3 & 1.7 & 1.7 & 2.1 & 1.9 & 1.8 & 1.4 \\
\hline $5 / 23 / 2007$ & 1.5 & 1.8 & 2.2 & 1.5 & 1.8 & 1.8 & 1.7 & 1.7 & 1.7 \\
\hline $6 / 27 / 2007$ & 1.7 & 1.7 & 2.0 & 1.6 & 1.7 & 1.5 & 1.4 & 1.4 & 1.2 \\
\hline $7 / 4 / 2007$ & 1.8 & 1.8 & 2.0 & 1.6 & 1.5 & & & & 0.6 \\
\hline $7 / 18 / 2007$ & 1.5 & 1.3 & 1.4 & 1.1 & 1.0 & 1.1 & 1.0 & 0.9 & 0.6 \\
\hline $8 / 15 / 2007$ & 1.8 & 1.7 & 2.3 & 1.4 & 1.4 & 1.3 & 1.1 & 1.1 & 0.7 \\
\hline $9 / 19 / 2007$ & 1.2 & 1.1 & 1.4 & 1.0 & 1.0 & & & & 0.5 \\
\hline Average & 1.2 & 1.3 & 1.4 & 1.2 & 1.2 & 1.2 & 1.1 & 1.1 & 1.0 \\
\hline $\begin{array}{l}\text { Standard } \\
\text { Deviation }\end{array}$ & 0.5 & 0.6 & 0.8 & 0.6 & 0.6 & 0.7 & 0.7 & 0.6 & 0.6 \\
\hline $\begin{array}{l}\text { Sampling } \\
\text { Date }\end{array}$ & $\begin{array}{c}\text { Point } \\
1\end{array}$ & $\begin{array}{c}\text { Point } \\
1-3\end{array}$ & $\begin{array}{l}\text { OW1- } \\
3-6 a_{B}\end{array}$ & $\begin{array}{l}\text { OW1- } \\
3-6 a_{M}\end{array}$ & $\begin{array}{l}\text { OW1- } \\
3-6 a_{S}\end{array}$ & $\begin{array}{l}\text { OW1- } \\
3-6 b_{B}\end{array}$ & $\begin{array}{l}\text { OW1- } \\
3-6 b_{M}\end{array}$ & $\begin{array}{l}\text { OW1- } \\
3-6 b_{S}\end{array}$ & $\begin{array}{c}\text { Point } \\
\text { 3-6 }\end{array}$ \\
\hline $10 / 19 / 2006$ & 26.7 & 27.4 & 27.5 & 27.4 & 29.3 & 28.0 & 27.8 & 29.4 & 27.5 \\
\hline $11 / 6 / 2006$ & 26.2 & 27.3 & 25.9 & 26.4 & 26.7 & 26.9 & 27.3 & 27.9 & 26.8 \\
\hline $12 / 19 / 2006$ & 24.8 & 24.5 & 22.5 & 22.4 & 22.3 & 22.8 & 22.5 & 22.8 & 25.8 \\
\hline $1 / 8 / 2007$ & 24.4 & 23.4 & 21.9 & 21.7 & 21.7 & 22.0 & 22.8 & 21.9 & 24.8 \\
\hline $1 / 17 / 2007$ & 23.8 & 22.6 & 21.4 & 21.3 & 21.4 & 21.5 & 21.1 & 21.1 & 25.6 \\
\hline $1 / 24 / 2007$ & 24.0 & 22.7 & 21.2 & 21.2 & 21.2 & 21.3 & 21.3 & 21.3 & 22.6 \\
\hline $1 / 30 / 2007$ & 23.9 & 23.1 & 20.8 & 20.6 & 20.6 & 20.7 & 20.7 & 20.7 & 24.2 \\
\hline $2 / 7 / 2007$ & 24.2 & 23.1 & 20.8 & 20.8 & 20.8 & 21.4 & 21.2 & 21.3 & 26.3 \\
\hline $3 / 28 / 2007$ & 25.2 & 26.2 & 21.1 & 21.1 & 22.0 & 21.5 & 21.9 & 21.9 & 27.1 \\
\hline $4 / 24 / 2007$ & 26.4 & 27.0 & 23.8 & 24.1 & 24.3 & 24.3 & 24.4 & 24.4 & 24.3 \\
\hline $5 / 9 / 2007$ & 26.1 & 26.5 & 24.3 & 24.3 & 24.4 & 25.6 & 25.0 & 25.6 & 24.4 \\
\hline $5 / 23 / 2007$ & 26.6 & 26.1 & 23.5 & 23.5 & 23.5 & 25.7 & 25.1 & 22.3 & 25.4 \\
\hline $6 / 27 / 2007$ & 27.2 & 27.7 & 24.3 & 24.3 & 24.4 & 24.6 & 24.6 & 24.6 & 25.4 \\
\hline $7 / 4 / 2007$ & 28.2 & 28.4 & 24.4 & 24.2 & 24.3 & & & & 26.4 \\
\hline $7 / 18 / 2007$ & 26.4 & 26.1 & 24.2 & 24.2 & 24.3 & 24.3 & 24.0 & 24.1 & 24.3 \\
\hline $8 / 15 / 2007$ & 27.1 & 25.7 & 24.6 & 24.5 & 24.5 & 25.5 & 25.7 & 25.9 & 24.4 \\
\hline $9 / 19 / 2007$ & 26.1 & 25.0 & 24.3 & 24.3 & 24.3 & & & & 23.5 \\
\hline
\end{tabular}




\begin{tabular}{|c|c|c|c|c|c|c|c|c|c|}
\hline Average & 25.7 & 25.5 & 23.3 & 23.3 & 23.5 & 23.7 & 23.7 & 23.7 & 25.2 \\
\hline $\begin{array}{l}\text { Standard } \\
\text { Deviation }\end{array}$ & 1.3 & 1.9 & 1.9 & 2.0 & 2.2 & 2.3 & 2.3 & 2.6 & 1.3 \\
\hline $\begin{array}{l}\text { Sampling } \\
\text { Date }\end{array}$ & $\begin{array}{c}\text { Point } \\
1\end{array}$ & $\begin{array}{c}\text { Point } \\
1-4\end{array}$ & $\begin{array}{l}\text { OW1- } \\
4-7 a_{B}\end{array}$ & $\begin{array}{l}\text { OW1- } \\
4-7 \mathbf{a}_{M}\end{array}$ & $\begin{array}{l}\text { OW1- } \\
4-7 a_{S}\end{array}$ & $\begin{array}{l}\text { OW1- } \\
4-7 b_{B}\end{array}$ & $\begin{array}{l}\text { OW1- } \\
4-7 b_{M}\end{array}$ & $\begin{array}{l}\text { OW1- } \\
4-7 b_{S}\end{array}$ & $\begin{array}{c}\text { Point } \\
4-7\end{array}$ \\
\hline $10 / 19 / 2006$ & 26.7 & 28.0 & 27.6 & 27.6 & 29.7 & 27.2 & 27.4 & 29.6 & 27.8 \\
\hline $11 / 6 / 2006$ & 26.2 & 27.7 & 25.8 & 26.1 & 26.2 & 25.9 & 26.5 & 27.0 & 26.5 \\
\hline $12 / 19 / 2006$ & 24.8 & 24.9 & 23.4 & 23.5 & 23.5 & 23.8 & 23.8 & 23.8 & 25.3 \\
\hline $1 / 8 / 2007$ & 24.4 & 24.0 & 22.1 & 22.1 & 22.0 & 22.3 & 22.1 & 22.0 & 24.4 \\
\hline $1 / 17 / 2007$ & 23.8 & 23.2 & 20.9 & 21.4 & 21.3 & 21.3 & 20.5 & 21.6 & 25.3 \\
\hline $1 / 24 / 2007$ & 24.0 & 22.9 & 21.7 & 21.4 & 21.4 & 20.8 & 20.9 & 20.9 & 23.4 \\
\hline $1 / 30 / 2007$ & 23.9 & 23.5 & 20.7 & 20.5 & 20.6 & 20.5 & 20.3 & 20.4 & 22.8 \\
\hline $2 / 7 / 2007$ & 24.2 & 23.6 & 21.1 & 21.1 & 21.1 & 20.9 & 20.8 & 20.8 & 24.2 \\
\hline $3 / 28 / 2007$ & 25.2 & 27.4 & 21.2 & 21.1 & 22.3 & 21.7 & 21.3 & 21.5 & 27.6 \\
\hline $4 / 24 / 2007$ & 26.4 & 26.3 & 23.8 & 23.9 & 23.9 & 23.9 & 23.8 & 23.8 & 25.2 \\
\hline $5 / 9 / 2007$ & 26.1 & 25.8 & 23.8 & 23.6 & 23.9 & 24.2 & 24.1 & 24.2 & 25.4 \\
\hline $5 / 23 / 2007$ & 26.6 & 26.7 & 23.6 & 23.6 & 23.7 & 24.5 & 24.1 & 24.2 & 25.2 \\
\hline $6 / 27 / 2007$ & 27.2 & 27.4 & 24.7 & 24.6 & 24.7 & 24.3 & 23.9 & 24.0 & 25.9 \\
\hline $7 / 4 / 2007$ & 28.2 & 24.9 & 24.7 & 24.5 & 24.5 & & & & 26.0 \\
\hline $7 / 18 / 2007$ & 26.4 & 26.1 & 24.4 & 24.3 & 24.5 & 23.8 & 23.7 & 23.8 & 23.8 \\
\hline $8 / 15 / 2007$ & 27.1 & 27.6 & 24.6 & 24.0 & 24.6 & 24.5 & 26.1 & 26.1 & 24.5 \\
\hline $9 / 19 / 2007$ & 26.1 & 25.9 & 24.4 & 24.4 & 24.3 & & & & 23.7 \\
\hline Average & 25.7 & 25.6 & 23.4 & 23.4 & 23.6 & 23.3 & 23.3 & 23.6 & 25.1 \\
\hline $\begin{array}{l}\text { Standard } \\
\text { Deviation }\end{array}$ & 1.3 & 1.7 & 1.9 & 1.9 & 2.2 & 2.0 & 2.3 & 2.5 & 1.4 \\
\hline
\end{tabular}




\begin{tabular}{|c|c|c|c|c|c|c|c|c|c|}
\hline Date & $\begin{array}{c}\text { Point } \\
1\end{array}$ & $\begin{array}{c}\text { Point } \\
1-2\end{array}$ & $\begin{array}{l}\text { OW1- } \\
2-5 a_{B}\end{array}$ & $\begin{array}{l}\text { OW1- } \\
2-5 a_{M}\end{array}$ & $\begin{array}{l}\text { OW1- } \\
2-5 a_{S}\end{array}$ & $\begin{array}{l}\text { OW1- } \\
2-5 b_{B}\end{array}$ & $\begin{array}{l}\text { OW1- } \\
2-5 b_{M}\end{array}$ & $\begin{array}{l}\text { OW1- } \\
2-5 b_{S}\end{array}$ & $\begin{array}{c}\text { Point } \\
2-5\end{array}$ \\
\hline $10 / 19 / 2006$ & 7.9 & 7.2 & 7.8 & 7.8 & 7.8 & 8.1 & 8.1 & 8.0 & 8.0 \\
\hline $11 / 6 / 2006$ & 8.0 & 7.4 & 7.8 & 7.8 & 7.8 & 7.5 & 7.5 & 7.5 & 7.8 \\
\hline $12 / 19 / 2006$ & 7.9 & 7.0 & 7.9 & 8.4 & 8.5 & 7.7 & 8.4 & 8.7 & 7.8 \\
\hline $1 / 8 / 2007$ & 8.1 & 6.9 & 7.3 & 7.2 & 7.2 & 7.2 & 7.6 & 7.3 & 7.5 \\
\hline $1 / 17 / 2007$ & 9.3 & 8.1 & 7.6 & 7.7 & 7.8 & 8.1 & 8.1 & 8.1 & 8.1 \\
\hline $1 / 24 / 2007$ & 9.5 & 8.1 & 8.0 & 8.3 & 8.2 & 8.0 & 8.4 & 8.3 & 7.9 \\
\hline $1 / 30 / 2007$ & 9.5 & 7.5 & 7.1 & 7.3 & 7.3 & 7.9 & 8.1 & 8.1 & 7.8 \\
\hline $2 / 7 / 2007$ & 9.3 & 7.4 & 7.5 & 7.6 & 7.6 & 7.9 & 8.0 & 8.0 & 8.3 \\
\hline $3 / 28 / 2007$ & 8.7 & 7.0 & 6.7 & 7.1 & 7.0 & 6.8 & 6.9 & 7.0 & 7.5 \\
\hline $4 / 24 / 2007$ & 8.9 & 7.0 & 6.7 & 7.2 & 7.1 & 7.0 & 7.2 & 7.3 & 7.4 \\
\hline $5 / 9 / 2007$ & 8.6 & 6.6 & 6.3 & 6.6 & 6.7 & 6.8 & 6.8 & 7.0 & 7.1 \\
\hline $5 / 23 / 2007$ & 8.8 & 6.6 & 6.3 & 6.7 & 6.7 & 6.7 & 6.8 & 6.8 & 7.0 \\
\hline $6 / 27 / 2007$ & 7.8 & 6.8 & 6.5 & 7.2 & 7.1 & 7.0 & 7.1 & 7.1 & 7.0 \\
\hline $7 / 4 / 2007$ & 7.3 & 6.8 & 6.6 & 7.1 & 7.4 & & & & 7.1 \\
\hline $7 / 18 / 2007$ & 7.7 & 7.0 & 6.8 & 7.1 & 7.0 & 7.2 & 7.2 & 7.2 & 6.9 \\
\hline $8 / 15 / 2007$ & 7.9 & 7.5 & 7.1 & 7.5 & 7.5 & 7.5 & 7.8 & 7.6 & 7.6 \\
\hline 9/19/2007 & 8.2 & 8.1 & 7.9 & 8.1 & 8.1 & & & & 8.2 \\
\hline Average & 8.4 & 7.2 & 7.2 & 7.4 & 7.5 & 7.4 & 7.6 & 7.6 & 7.6 \\
\hline $\begin{array}{l}\text { Standard } \\
\text { Deviation }\end{array}$ & 0.7 & 0.5 & 0.6 & 0.5 & 0.5 & 0.5 & 0.6 & 0.6 & 0.5 \\
\hline $\begin{array}{l}\text { Sampling } \\
\text { Date }\end{array}$ & $\begin{array}{c}\text { Point } \\
1\end{array}$ & $\begin{array}{c}\text { Point } \\
1-3\end{array}$ & $\begin{array}{l}\text { OW1- } \\
\text { 3-6a }\end{array}$ & $\begin{array}{l}\text { OW1- } \\
3-6 a_{M}\end{array}$ & $\begin{array}{l}\text { OW1- } \\
3-6 a_{s}\end{array}$ & $\begin{array}{l}\text { OW1- } \\
\text { 3-6b }\end{array}$ & $\begin{array}{l}\text { OW1- } \\
3-6 b_{M}\end{array}$ & $\begin{array}{l}\text { OW1- } \\
3-6 b_{S}\end{array}$ & $\begin{array}{c}\text { Point } \\
\text { 3-6 }\end{array}$ \\
\hline $10 / 19 / 2006$ & 7.9 & 7.1 & 7.6 & 7.7 & 7.7 & 7.7 & 7.8 & 7.8 & 7.9 \\
\hline $11 / 6 / 2006$ & 8.0 & 7.2 & 8.5 & 8.6 & 8.5 & 8.0 & 8.1 & 8.1 & 8.6 \\
\hline $12 / 19 / 2006$ & 7.9 & 6.9 & 7.9 & 7.8 & 8.0 & 8.4 & 8.4 & 8.6 & 8.0 \\
\hline $1 / 8 / 2007$ & 8.1 & 6.9 & 7.0 & 7.1 & 7.1 & 7.4 & 7.6 & 7.5 & 7.5 \\
\hline $1 / 17 / 2007$ & 9.3 & 8.4 & 8.1 & 8.1 & 8.1 & 7.6 & 7.9 & 8.1 & 8.2 \\
\hline $1 / 24 / 2007$ & 9.5 & 8.1 & 7.6 & 8.1 & 8.1 & 7.9 & 8.4 & 8.4 & 7.8 \\
\hline $1 / 30 / 2007$ & 9.5 & 7.5 & 7.1 & 7.2 & 7.3 & 7.4 & 7.8 & 7.8 & 7.8 \\
\hline $2 / 7 / 2007$ & 9.3 & 7.5 & 7.6 & 7.4 & 7.6 & 7.6 & 7.7 & 7.8 & 8.2 \\
\hline $3 / 28 / 2007$ & 8.7 & 6.9 & 6.6 & 6.9 & 7.0 & 6.8 & 7.0 & 6.9 & 7.5 \\
\hline $4 / 24 / 2007$ & 8.9 & 6.9 & 6.7 & 7.0 & 7.0 & 6.6 & 7.1 & 7.1 & 7.0 \\
\hline $5 / 9 / 2007$ & 8.6 & 6.6 & 6.5 & 6.9 & 6.9 & 6.6 & 7.2 & 7.2 & 6.7 \\
\hline $5 / 23 / 2007$ & 8.8 & 6.6 & 6.5 & 6.5 & 6.6 & 6.6 & 7.1 & 6.9 & 6.9 \\
\hline $6 / 27 / 2007$ & 7.8 & 6.8 & 6.5 & 6.7 & 6.7 & 6.7 & 6.9 & 6.9 & 6.8 \\
\hline $7 / 4 / 2007$ & 7.3 & 6.9 & 6.7 & 6.8 & 6.9 & & & & 7.0 \\
\hline $7 / 18 / 2007$ & 7.7 & 6.9 & 6.9 & 7.1 & 7.1 & 6.8 & 7.1 & 7.0 & 6.8 \\
\hline $8 / 15 / 2007$ & 7.9 & 7.5 & 7.5 & 7.6 & 7.6 & 7.7 & 8.0 & 8.0 & 7.7 \\
\hline $9 / 19 / 2007$ & 8.2 & 8.1 & 7.8 & 8.1 & 8.1 & & & & 8.1 \\
\hline
\end{tabular}




\begin{tabular}{|c|c|c|c|c|c|c|c|c|c|}
\hline Average & 8.4 & 7.2 & 7.2 & 7.4 & 7.4 & 7.3 & 7.6 & 7.6 & 7.6 \\
\hline $\begin{array}{l}\text { Standard } \\
\text { Deviation }\end{array}$ & 0.7 & 0.5 & 0.6 & 0.6 & 0.6 & 0.6 & 0.5 & 0.6 & 0.6 \\
\hline $\begin{array}{c}\text { Sampling } \\
\text { Date }\end{array}$ & $\begin{array}{c}\text { Point } \\
1\end{array}$ & $\begin{array}{l}\text { Point } \\
1-4\end{array}$ & $\begin{array}{l}\text { OW1- } \\
4-7 \mathrm{a}_{B}\end{array}$ & $\begin{array}{l}\text { OW1- } \\
4-7 a_{M}\end{array}$ & $\begin{array}{l}\text { OW1- } \\
4-7 a_{s}\end{array}$ & $\begin{array}{l}\text { OW1- } \\
4-7 b_{B}\end{array}$ & $\begin{array}{l}\text { OW1- } \\
4-7 b_{M}\end{array}$ & $\begin{array}{l}\text { OW1- } \\
4-7 b_{S}\end{array}$ & $\begin{array}{c}\text { Point } \\
4-7\end{array}$ \\
\hline $10 / 19 / 2006$ & 7.9 & 7.2 & 7.4 & 7.7 & 7.7 & 7.7 & 7.7 & 8.0 & 7.8 \\
\hline $11 / 6 / 2006$ & 8.0 & 7.4 & 7.7 & 7.7 & 7.7 & 7.6 & 7.7 & 7.6 & 7.5 \\
\hline $12 / 19 / 2006$ & 7.9 & 7.2 & 6.7 & 7.0 & 7.1 & 7.4 & 7.6 & 7.6 & 7.6 \\
\hline $1 / 8 / 2007$ & 8.1 & 7.0 & 7.1 & 7.1 & 7.1 & 7.4 & 7.4 & 7.4 & 7.4 \\
\hline $1 / 17 / 2007$ & 9.3 & 7.7 & 7.6 & 7.6 & 7.6 & 8.0 & 7.9 & 7.9 & 8.2 \\
\hline $1 / 24 / 2007$ & 9.5 & 7.9 & 7.5 & 7.7 & 7.8 & 7.9 & 7.9 & 8.0 & 8.0 \\
\hline $1 / 30 / 2007$ & 9.5 & 7.4 & 6.8 & 7.1 & 7.1 & 7.5 & 7.8 & 7.7 & 7.8 \\
\hline $2 / 7 / 2007$ & 9.3 & 7.4 & 7.4 & 7.4 & 7.4 & 7.6 & 7.7 & 7.7 & 8.0 \\
\hline $3 / 28 / 2007$ & 8.7 & 7.0 & 7.0 & 7.0 & 7.2 & 7.0 & 7.1 & 7.1 & 7.8 \\
\hline $4 / 24 / 2007$ & 8.9 & 6.9 & 6.5 & 6.9 & 6.9 & 6.8 & 7.0 & 7.1 & 7.1 \\
\hline $5 / 9 / 2007$ & 8.6 & 6.6 & 6.5 & 6.7 & 6.8 & 6.3 & 6.9 & 7.0 & 6.8 \\
\hline $5 / 23 / 2007$ & 8.8 & 6.5 & 6.3 & 6.4 & 6.5 & 6.3 & 6.8 & 6.8 & 7.0 \\
\hline $6 / 27 / 2007$ & 7.8 & 6.6 & 6.5 & $6 . .77$ & 6.7 & 6.6 & 6.8 & 6.8 & 7.1 \\
\hline $7 / 4 / 2007$ & 7.3 & 6.8 & 6.2 & 6.4 & 6.4 & & & & 7.1 \\
\hline $7 / 18 / 2007$ & 7.7 & 6.9 & 6.6 & 6.9 & 7.0 & 6.8 & 6.8 & 6.8 & 7.0 \\
\hline $8 / 15 / 2007$ & 7.9 & 7.5 & 7.5 & 7.5 & 7.7 & 7.7 & 8.2 & 8.3 & 7.7 \\
\hline 9/19/2007 & 8.2 & 8.1 & 7.8 & 8.1 & 8.1 & & & & 7.9 \\
\hline Average & 8.4 & 7.2 & 7.0 & 7.2 & 7.2 & 7.2 & 7.4 & 7.4 & 7.5 \\
\hline $\begin{array}{l}\text { Standard } \\
\text { Deviation }\end{array}$ & 0.7 & 0.5 & 0.5 & 0.5 & 0.5 & 0.6 & 0.5 & 0.5 & 0.4 \\
\hline
\end{tabular}




\begin{tabular}{|c|c|c|c|c|c|c|c|c|c|}
\hline Date & $\begin{array}{c}\text { Point } \\
1\end{array}$ & $\begin{array}{c}\text { Point } \\
1-2\end{array}$ & $\begin{array}{l}\text { OW1- } \\
2-5 a_{B}\end{array}$ & $\begin{array}{l}\text { OW1- } \\
2-5 a_{M}\end{array}$ & $\begin{array}{l}\text { OW1- } \\
2-5 a_{S}\end{array}$ & $\begin{array}{l}\text { OW1- } \\
2-5 b_{B}\end{array}$ & $\begin{array}{l}\text { OW1- } \\
2-5 b_{M}\end{array}$ & $\begin{array}{l}\text { OW1- } \\
2-5 b_{S}\end{array}$ & $\begin{array}{c}\text { Point } \\
2-5\end{array}$ \\
\hline $10 / 19 / 2006$ & -225.9 & -152.8 & 2.9 & 35.9 & 80.9 & 67.0 & 60.3 & 100.0 & 190.0 \\
\hline $11 / 6 / 2006$ & -170.6 & -141.5 & 56.3 & 36.8 & 43.2 & 110.6 & 47.7 & 100.8 & 273.2 \\
\hline $12 / 19 / 2006$ & -177.0 & -187.9 & -132.8 & -168.8 & -164.7 & -107.0 & -127.0 & -110.3 & 179.9 \\
\hline $1 / 8 / 2007$ & -233.4 & -234.1 & -183.9 & -238.6 & -228.9 & -150.0 & -161.0 & -173.4 & 75.7 \\
\hline $1 / 17 / 2007$ & -187.0 & -179.0 & -177.8 & -208.1 & -204.9 & -127.6 & -134.4 & -135.1 & 154.5 \\
\hline $1 / 24 / 2007$ & -164.0 & -207.0 & -158.5 & -200.9 & -195.8 & -98.9 & -127.6 & -125.5 & 133.9 \\
\hline $1 / 30 / 2007$ & -175.2 & -208.5 & -152.7 & -182.4 & -176.5 & -54.9 & -64.8 & -63.2 & 114.4 \\
\hline $2 / 7 / 2007$ & -181.7 & -210.0 & -182.8 & -211.9 & -204.3 & -115.5 & -129.8 & -122.8 & 197.6 \\
\hline $3 / 28 / 2007$ & -155.4 & -254.9 & -209.9 & -262.7 & -250.6 & -229.5 & -257.9 & -249.8 & -181.4 \\
\hline $4 / 24 / 2007$ & -279.1 & -269.7 & -293.3 & -305.8 & -303.7 & -273.8 & -299.2 & -291.8 & -130.0 \\
\hline $5 / 9 / 2007$ & -279.2 & -273.8 & -261.8 & -274.3 & -273.9 & -268.9 & -284.7 & -282.7 & -61.5 \\
\hline $5 / 23 / 2007$ & -270.8 & -265.5 & -175.7 & -237.6 & -230.0 & -232.6 & -242.9 & -246.0 & -97.9 \\
\hline $6 / 27 / 2007$ & -197.5 & -176.4 & -172.8 & -163.8 & -174.0 & -87.8 & -123.5 & -110.8 & 45.8 \\
\hline $7 / 4 / 2007$ & -166.5 & -172.4 & -128.7 & -147.6 & -154.2 & & & & -38.4 \\
\hline $7 / 18 / 2007$ & -165.7 & -174.0 & -125.9 & -140.7 & -134.4 & -58.4 & -88.3 & -80.0 & 161.2 \\
\hline $8 / 15 / 2007$ & -186.1 & -161.7 & -117.9 & -142.4 & -140.9 & -42.5 & -85.2 & -69.3 & -14.2 \\
\hline $9 / 19 / 2007$ & -175.6 & -160.5 & -113.7 & -125.7 & -121.0 & & & & 31.8 \\
\hline Average & -199.4 & -201.7 & -148.8 & -172.9 & -166.7 & -111.3 & -134.6 & -124.0 & 60.8 \\
\hline $\begin{array}{l}\text { Standard } \\
\text { Deviation }\end{array}$ & 42.0 & 43.4 & 83.1 & 93.8 & 99.5 & 111.5 & 106.3 & 118.4 & 130.8 \\
\hline $\begin{array}{l}\text { Sampling } \\
\text { Date }\end{array}$ & $\begin{array}{c}\text { Point } \\
1\end{array}$ & $\begin{array}{c}\text { Point } \\
1-3\end{array}$ & $\begin{array}{l}\text { OW1- } \\
3-6 a_{B}\end{array}$ & $\begin{array}{l}\text { OW1- } \\
3-6 a_{M}\end{array}$ & $\begin{array}{l}\text { OW1- } \\
3-6 a_{S}\end{array}$ & $\begin{array}{l}\text { OW1- } \\
3-6 b_{B}\end{array}$ & $\begin{array}{l}\text { OW1- } \\
3-6 b_{M}\end{array}$ & $\begin{array}{l}\text { OW1- } \\
3-6 b_{S}\end{array}$ & $\begin{array}{c}\text { Point } \\
3-6\end{array}$ \\
\hline $10 / 19 / 2006$ & -225.9 & -219.6 & 46.0 & 32.1 & 130.0 & -70.5 & -16.5 & 61.0 & 187.3 \\
\hline $11 / 6 / 2006$ & -170.6 & -174.2 & 60.8 & 27.1 & 38.9 & 64.0 & 60.6 & 60.1 & 242.1 \\
\hline $12 / 19 / 2006$ & -177.0 & -166.9 & -141.5 & -161.7 & -175.2 & -158.8 & -160.6 & -142.5 & 159.3 \\
\hline $1 / 8 / 2007$ & -233.4 & -230.0 & -218.9 & -247.6 & -236.9 & -181.0 & -181.2 & -193.5 & 54.4 \\
\hline $1 / 17 / 2007$ & -187.0 & -176.2 & -121.4 & -186.4 & -173.3 & -154.0 & -174.4 & -184.1 & 95.2 \\
\hline $1 / 24 / 2007$ & -164.0 & -199.2 & -149.5 & -176.7 & -178.5 & -155.7 & -148.7 & -163.7 & 57.8 \\
\hline $1 / 30 / 2007$ & -175.2 & -199.0 & -145.0 & -154.7 & -160.9 & -107.9 & -120.1 & -121.7 & -34.7 \\
\hline $2 / 7 / 2007$ & -181.7 & -210.8 & -151.7 & -163.7 & -160.9 & -121.9 & -148.2 & -147.7 & 139.6 \\
\hline $3 / 28 / 2007$ & -155.4 & -239.9 & -217.9 & -241.0 & -239.2 & -232.2 & -267.5 & -260.5 & -189.0 \\
\hline $4 / 24 / 2007$ & -279.1 & -231.0 & 280.9 & -282.8 & -286.0 & -278.6 & -290.1 & -287.6 & -62.4 \\
\hline $5 / 9 / 2007$ & -279.2 & -250.1 & -262.3 & -267.5 & -267.5 & -230.0 & -261.5 & -264.1 & -94.1 \\
\hline $5 / 23 / 2007$ & -270.8 & -266.1 & -141.7 & -155.5 & -156.0 & -116.0 & -235.0 & -242.8 & -126.0 \\
\hline $6 / 27 / 2007$ & -197.5 & -168.0 & -160.2 & -179.0 & -178.3 & -138.7 & -152.3 & -149.1 & -64.4 \\
\hline $7 / 4 / 2007$ & -166.5 & -172.7 & -159.2 & -171.0 & -173.5 & & & & -63.7 \\
\hline $7 / 18 / 2007$ & -165.7 & -162.9 & -172.1 & -126.9 & -189.4 & -107.8 & -136.6 & -132.5 & -61.6 \\
\hline $8 / 15 / 2007$ & -186.1 & -151.8 & -124.0 & -139.1 & -137.4 & -81.2 & -71.0 & -83.2 & -43.6 \\
\hline 9/19/2007 & -175.6 & -163.7 & -144.7 & -154.3 & -156.9 & & & & -9.0 \\
\hline
\end{tabular}




\begin{tabular}{|c|c|c|c|c|c|c|c|c|c|}
\hline Average & -199.4 & -198.9 & -113.1 & -161.7 & -158.9 & -138.0 & -153.5 & -150.1 & 11.0 \\
\hline $\begin{array}{l}\text { Standard } \\
\text { Deviation }\end{array}$ & 42.0 & 35.3 & 129.8 & 85.1 & 101.8 & 80.7 & 94.1 & 103.6 & 120.2 \\
\hline $\begin{array}{l}\text { Sampling } \\
\text { Date }\end{array}$ & $\begin{array}{c}\text { Point } \\
1\end{array}$ & $\begin{array}{c}\text { Point } \\
1-4\end{array}$ & $\begin{array}{l}\text { OW1- } \\
4-7 a_{B}\end{array}$ & $\begin{array}{l}\text { OW1- } \\
4-7 a_{M}\end{array}$ & $\begin{array}{l}\text { OW1- } \\
4-7 a_{s}\end{array}$ & $\begin{array}{l}\text { OW1- } \\
4-7 b_{B}\end{array}$ & $\begin{array}{l}\text { OW1- } \\
4-7 b_{M}\end{array}$ & $\begin{array}{l}\text { OW1- } \\
4-7 b_{s}\end{array}$ & $\begin{array}{c}\text { Point } \\
4-7\end{array}$ \\
\hline $10 / 19 / 2006$ & -225.9 & -164.5 & -50.0 & -17.2 & 125.2 & 65.2 & 63.5 & 70.1 & 193.2 \\
\hline $11 / 6 / 2006$ & -170.6 & -153.6 & 38.2 & 32.8 & 31.9 & 80.1 & 76.6 & 74.6 & 190.2 \\
\hline $12 / 19 / 2006$ & -177.0 & -170.3 & -150.3 & -181.4 & -207.6 & -121.9 & -152.3 & -173.9 & 130.1 \\
\hline $1 / 8 / 2007$ & -233.4 & -210.8 & -201.9 & -253.1 & -237.5 & -160.0 & -180.5 & -177.1 & -79.9 \\
\hline $1 / 17 / 2007$ & -187.0 & -179.3 & -178.2 & -204.9 & -199.8 & -124.5 & -159.3 & -151.8 & 7.9 \\
\hline $1 / 24 / 2007$ & -164.0 & -166.8 & -167.5 & -195.5 & -195.0 & -133.9 & -166.7 & -152.6 & -25.9 \\
\hline $1 / 30 / 2007$ & -175.2 & -182.3 & -146.3 & -170.4 & -170.5 & -120.4 & -150.9 & -142.0 & -43.5 \\
\hline $2 / 7 / 2007$ & -181.7 & -191.7 & -171.0 & -196.0 & -184.7 & -164.0 & -179.1 & -176.5 & -53.2 \\
\hline $3 / 28 / 2007$ & -155.4 & -239.9 & -212.7 & -237.1 & -227.8 & -222.9 & -260.9 & -248.9 & -188.2 \\
\hline $4 / 24 / 2007$ & -279.1 & -193.9 & -263.9 & -290.7 & -288.8 & -279.5 & -305.7 & -304.9 & -45.4 \\
\hline $5 / 9 / 2007$ & -279.2 & -179.6 & -260.5 & -269.6 & -270.3 & -262.0 & -276.1 & -273.4 & -61.9 \\
\hline $5 / 23 / 2007$ & -270.8 & -251.9 & -150.4 & -153.3 & -155.2 & -186.9 & -214.4 & -217.8 & -70.9 \\
\hline $6 / 27 / 2007$ & -197.5 & -121.8 & -130.5 & -160.6 & -153.9 & -98.9 & -130.3 & -122.2 & -42.4 \\
\hline $7 / 4 / 2007$ & -166.5 & -64.5 & -109.2 & -137.7 & -131.3 & & & & -62.6 \\
\hline $7 / 18 / 2007$ & -165.7 & -141.9 & -126.4 & -170.0 & -160.4 & -64.5 & -79.7 & -75.7 & -35.8 \\
\hline $8 / 15 / 2007$ & -186.1 & -115.5 & -31.5 & -27.2 & -23.1 & -23.0 & -170.8 & -180.3 & -23.1 \\
\hline $9 / 19 / 2007$ & -175.6 & -131.6 & -142.7 & -157.0 & -158.5 & & & & 17.1 \\
\hline Average & -199.4 & -168.2 & -144.4 & -164.1 & -153.4 & -121.1 & -152.4 & -150.2 & -11.4 \\
\hline $\begin{array}{l}\text { Standard } \\
\text { Deviation }\end{array}$ & 42.0 & 45.8 & 77.0 & 88.2 & 106.7 & 104.1 & 107.4 & 107.5 & 98.2 \\
\hline
\end{tabular}




\begin{tabular}{|c|c|c|c|c|c|c|c|c|c|}
\hline Date & $\begin{array}{c}\text { Point } \\
1\end{array}$ & $\begin{array}{c}\text { Point } \\
1-2\end{array}$ & $\begin{array}{l}\text { OW1- } \\
2-5 a_{B}\end{array}$ & $\begin{array}{l}\text { OW1- } \\
2-5 a_{M}\end{array}$ & $\begin{array}{l}\text { OW1- } \\
2-5 a_{S}\end{array}$ & $\begin{array}{l}\text { OW1- } \\
2-5 b_{B}\end{array}$ & $\begin{array}{l}\text { OW1- } \\
2-5 b_{M}\end{array}$ & $\begin{array}{l}\text { OW1- } \\
2-5 b_{S}\end{array}$ & $\begin{array}{c}\text { Point } \\
2-5\end{array}$ \\
\hline $10 / 19 / 2006$ & 0.2 & 0.1 & 6.9 & 7.1 & 6.9 & 7.6 & 8.1 & 9.2 & 3.4 \\
\hline $11 / 6 / 2006$ & 0.4 & 0.2 & 3.8 & 3.9 & 4.3 & 2.8 & 3.8 & 4.4 & 2.9 \\
\hline $12 / 19 / 2006$ & 0.2 & 0.2 & 0.1 & 0.2 & 0.3 & 0.1 & 1.0 & 2.8 & 2.8 \\
\hline $1 / 8 / 2007$ & 4.5 & 3.8 & 1.8 & 3.2 & 3.4 & 3.7 & 5.3 & 2.8 & 1.7 \\
\hline $1 / 17 / 2007$ & 0.2 & 0.2 & 0.2 & 0.1 & 0.1 & 0.3 & 0.3 & 0.4 & 2.5 \\
\hline $1 / 24 / 2007$ & 0.2 & 0.1 & 0.3 & 0.1 & 0.2 & 0.8 & 0.4 & 0.6 & 0.4 \\
\hline $1 / 30 / 2007$ & 0.4 & 0.9 & 0.5 & 0.1 & 0.1 & 0.2 & 0.3 & 0.3 & 1.7 \\
\hline $2 / 7 / 2007$ & 0.1 & 0.3 & 0.2 & 0.1 & 0.1 & 0.1 & 0.1 & 0.1 & 2.7 \\
\hline $3 / 28 / 2007$ & 0.1 & 0.8 & 0.1 & 0.1 & 0.1 & 0.1 & 0.1 & 0.1 & 0.2 \\
\hline $4 / 24 / 2007$ & 0.1 & 0.6 & 0.3 & 0.3 & 0.3 & 0.3 & 0.1 & 0.1 & 0.2 \\
\hline $5 / 9 / 2007$ & 0.1 & 0.5 & 0.9 & 0.3 & 0.4 & 0.2 & 0.1 & 0.1 & 0.3 \\
\hline $5 / 23 / 2007$ & 0.1 & 0.3 & 0.4 & 0.3 & 1.0 & 0.2 & 0.1 & 0.1 & 0.6 \\
\hline $6 / 27 / 2007$ & 0.1 & 0.2 & 0.7 & 0.5 & 1.2 & 0.2 & 0.1 & 0.1 & 0.3 \\
\hline $7 / 4 / 2007$ & 0.2 & 0.2 & 0.5 & 0.5 & 0.7 & & & & 0.4 \\
\hline $7 / 18 / 2007$ & 0.2 & 0.1 & 0.3 & 0.1 & 0.2 & 0.7 & 0.1 & 0.2 & 0.4 \\
\hline $8 / 15 / 2007$ & 0.2 & 0.3 & 1.3 & 0.5 & 0.7 & 0.4 & 0.3 & 0.2 & 0.3 \\
\hline $9 / 19 / 2007$ & 0.8 & 0.5 & 0.1 & 0.2 & 0.4 & & & & 1.7 \\
\hline Average & 0.5 & 0.5 & 1.1 & 1.0 & 1.2 & 1.2 & 1.3 & 1.4 & 1.3 \\
\hline $\begin{array}{l}\text { Standard } \\
\text { Deviation }\end{array}$ & 1.1 & 0.9 & 1.8 & 1.9 & 1.9 & 2.1 & 2.4 & 2.5 & 1.2 \\
\hline $\begin{array}{l}\text { Sampling } \\
\text { Date }\end{array}$ & $\begin{array}{c}\text { Point } \\
1\end{array}$ & $\begin{array}{c}\text { Point } \\
1-3\end{array}$ & $\begin{array}{l}\text { OW1- } \\
3-6 a_{B}\end{array}$ & $\begin{array}{l}\text { OW1- } \\
3-6 a_{M}\end{array}$ & $\begin{array}{l}\text { OW1- } \\
3-6 a_{s}\end{array}$ & $\begin{array}{l}\text { OW1- } \\
3-6 b_{B}\end{array}$ & $\begin{array}{l}\text { OW1- } \\
3-6 b_{M}\end{array}$ & $\begin{array}{l}\text { OW1- } \\
3-6 b_{S}\end{array}$ & $\begin{array}{c}\text { Point } \\
\text { 3-6 }\end{array}$ \\
\hline $10 / 19 / 2006$ & 0.2 & 0.1 & 3.5 & 12.0 & 5.2 & 7.3 & 6.9 & 6.8 & 3.9 \\
\hline $11 / 6 / 2006$ & 0.4 & 0.1 & 6.4 & 6.6 & 7.6 & 4.3 & 5.0 & 5.8 & 5.0 \\
\hline $12 / 19 / 2006$ & 0.2 & 0.3 & 0.3 & 0.2 & 0.2 & 0.1 & 0.1 & 1.3 & 2.6 \\
\hline $1 / 8 / 2007$ & 4.5 & 2.7 & 4.3 & 4.8 & 4.5 & 5.2 & 4.5 & 5.1 & 3.1 \\
\hline $1 / 17 / 2007$ & 0.2 & 0.2 & 2.8 & 1.5 & 1.5 & 0.2 & 0.1 & 0.1 & 2.0 \\
\hline $1 / 24 / 2007$ & 0.2 & 0.2 & 0.5 & 0.4 & 0.4 & 0.6 & 0.7 & 0.7 & 1.0 \\
\hline $1 / 30 / 2007$ & 0.4 & 0.2 & 0.2 & 0.1 & 0.1 & 0.3 & 0.1 & 0.2 & 0.2 \\
\hline $2 / 7 / 2007$ & 0.1 & 0.2 & 0.2 & 0.1 & 0.1 & 0.2 & 0.1 & 0.2 & 0.8 \\
\hline $3 / 28 / 2007$ & 0.1 & 0.2 & 0.1 & 0.1 & 0.1 & 0.1 & 0.1 & 0.1 & 0.2 \\
\hline $4 / 24 / 2007$ & 0.1 & 0.8 & 0.5 & 0.4 & 0.6 & 0.2 & 0.0 & 0.1 & 0.2 \\
\hline 5/9/2007 & 0.1 & 0.3 & 0.3 & 0.7 & 0.3 & 0.2 & 0.1 & 0.1 & 0.2 \\
\hline $5 / 23 / 2007$ & 0.1 & 0.3 & 0.6 & 0.1 & 0.1 & 0.2 & 0.1 & 0.2 & 0.2 \\
\hline $6 / 27 / 2007$ & 0.1 & 0.2 & 0.8 & 0.4 & 0.5 & 0.1 & 0.1 & 0.1 & 0.2 \\
\hline $7 / 4 / 2007$ & 0.2 & 0.1 & 0.4 & 0.4 & 0.4 & & & & 0.1 \\
\hline $7 / 18 / 2007$ & 0.2 & 0.2 & 0.1 & 0.1 & 0.1 & 0.4 & 0.1 & 0.1 & 0.3 \\
\hline $8 / 15 / 2007$ & 0.2 & 0.3 & 0.7 & 0.1 & 0.1 & 0.3 & 0.4 & 3.4 & 0.6 \\
\hline $9 / 19 / 2007$ & 0.8 & 0.6 & 0.6 & 0.2 & 0.4 & & & & 0.9 \\
\hline
\end{tabular}




\begin{tabular}{|c|c|c|c|c|c|c|c|c|c|}
\hline Average & 0.5 & 0.4 & 1.3 & 1.7 & 1.3 & 1.3 & 1.2 & 1.6 & 1.3 \\
\hline $\begin{array}{l}\text { Standard } \\
\text { Deviation }\end{array}$ & 1.1 & 0.6 & 1.8 & 3.2 & 2.2 & 2.3 & 2.3 & 2.4 & 1.5 \\
\hline $\begin{array}{l}\text { Sampling } \\
\text { Date }\end{array}$ & $\begin{array}{c}\text { Point } \\
1\end{array}$ & $\begin{array}{l}\text { Point } \\
1-4\end{array}$ & $\begin{array}{l}\text { OW1- } \\
4-7 \mathbf{a}_{B}\end{array}$ & $\begin{array}{l}\text { OW1- } \\
4-7 a_{M}\end{array}$ & $\begin{array}{l}\text { OW1- } \\
4-7 a_{s}\end{array}$ & $\begin{array}{l}\text { OW1- } \\
4-7 b_{B}\end{array}$ & $\begin{array}{l}\text { OW1- } \\
4-7 b_{M}\end{array}$ & $\begin{array}{l}\text { OW1- } \\
4-7 b_{S}\end{array}$ & $\begin{array}{c}\text { Point } \\
4-7\end{array}$ \\
\hline $10 / 19 / 2006$ & 0.2 & 0.2 & 2.2 & 5.2 & 5.2 & 5.8 & 6.6 & 9.7 & 3.1 \\
\hline $11 / 6 / 2006$ & 0.4 & 0.1 & 1.0 & 1.8 & 4.2 & 2.5 & 4.6 & 7.0 & 0.6 \\
\hline $12 / 19 / 2006$ & 0.2 & 0.4 & 0.0 & 0.0 & 0.2 & 0.1 & 0.0 & 0.2 & 1.6 \\
\hline $1 / 8 / 2007$ & 4.5 & 1.6 & 6.0 & 5.7 & 5.7 & 2.5 & 5.0 & 3.0 & 5.6 \\
\hline $1 / 17 / 2007$ & 0.2 & 0.2 & 0.5 & 0.2 & 0.3 & 0.3 & 0.1 & 0.1 & 1.9 \\
\hline $1 / 24 / 2007$ & 0.2 & 0.2 & 0.4 & 0.1 & 0.1 & 0.3 & 0.1 & 0.2 & 0.2 \\
\hline $1 / 30 / 2007$ & 0.4 & 0.2 & 0.7 & 0.3 & 0.3 & 0.5 & 0.2 & 0.2 & 0.4 \\
\hline $2 / 7 / 2007$ & 0.1 & 0.2 & 0.4 & 0.2 & 0.2 & 0.1 & 0.1 & 0.1 & 0.2 \\
\hline $3 / 28 / 2007$ & 0.1 & 0.1 & 0.1 & 0.1 & 0.1 & 0.1 & 0.1 & 0.1 & 0.2 \\
\hline $4 / 24 / 2007$ & 0.1 & 0.1 & 0.9 & 0.5 & 0.7 & 0.2 & 0.1 & 0.1 & 0.1 \\
\hline $5 / 9 / 2007$ & 0.1 & 0.2 & 1.1 & 0.5 & 0.8 & 0.3 & 0.1 & 0.1 & 0.3 \\
\hline $5 / 23 / 2007$ & 0.1 & 0.2 & 0.1 & 0.1 & 0.1 & 0.5 & 0.1 & 0.2 & 0.2 \\
\hline $6 / 27 / 2007$ & 0.1 & 0.2 & 0.3 & 0.4 & 0.3 & 0.2 & 0.1 & 0.1 & 0.4 \\
\hline $7 / 4 / 2007$ & 0.2 & 0.2 & 0.3 & 0.3 & 0.3 & & & & 0.4 \\
\hline $7 / 18 / 2007$ & 0.2 & 0.2 & 0.2 & 0.1 & 0.1 & 0.6 & 0.1 & 0.2 & 0.5 \\
\hline $8 / 15 / 2007$ & 0.2 & 0.3 & 0.1 & 0.2 & 0.2 & 0.2 & 0.8 & 0.7 & 0.2 \\
\hline 9/19/2007 & 0.8 & 1.5 & 0.1 & 0.2 & 0.1 & & & & 0.9 \\
\hline Average & 0.5 & 0.4 & 0.8 & 0.9 & 1.1 & 0.9 & 1.2 & 1.5 & 1.0 \\
\hline $\begin{array}{l}\text { Standard } \\
\text { Deviation }\end{array}$ & 1.1 & 0.5 & 1.4 & 1.7 & 1.9 & 1.6 & 2.2 & 2.9 & 1.4 \\
\hline
\end{tabular}




\begin{tabular}{|c|c|c|c|c|c|c|c|c|c|}
\hline Date & $\begin{array}{c}\text { Point } \\
1\end{array}$ & $\begin{array}{l}\text { Point } \\
1-2\end{array}$ & $\begin{array}{l}\text { OW1- } \\
2-5 a_{B}\end{array}$ & $\begin{array}{l}\text { OW1- } \\
2-5 a_{M}\end{array}$ & $\begin{array}{l}\text { OW1- } \\
2-5 a_{S}\end{array}$ & $\begin{array}{l}\text { OW1- } \\
2-5 b_{B}\end{array}$ & $\begin{array}{l}\text { OW1- } \\
2-5 b_{M}\end{array}$ & $\begin{array}{l}\text { OW1- } \\
2-5 b_{s}\end{array}$ & $\begin{array}{c}\text { Point } \\
2-5\end{array}$ \\
\hline $10 / 19 / 2006$ & 0.6 & 0.7 & 0.6 & 0.6 & 0.6 & 0.3 & 0.3 & 0.3 & 0.3 \\
\hline $11 / 6 / 2006$ & 0.7 & 0.6 & 0.4 & 0.5 & 0.4 & 0.4 & 0.3 & 0.4 & 0.3 \\
\hline $12 / 19 / 2006$ & 0.8 & 0.8 & 0.3 & 0.6 & 0.5 & 0.7 & 0.6 & 0.5 & 0.5 \\
\hline $1 / 8 / 2007$ & 0.8 & 0.9 & 0.8 & 0.8 & 0.8 & 0.7 & 0.7 & 0.7 & 0.8 \\
\hline $1 / 17 / 2007$ & 0.7 & 0.7 & 0.8 & 0.8 & 0.7 & 0.7 & 0.7 & 0.7 & 0.7 \\
\hline $1 / 24 / 2007$ & 0.7 & 0.7 & 0.7 & 0.7 & 0.7 & 0.7 & 0.7 & 0.7 & 0.8 \\
\hline $1 / 30 / 2007$ & 0.7 & 0.8 & 0.8 & 0.8 & 0.8 & 0.7 & 0.7 & 0.7 & 0.8 \\
\hline $2 / 7 / 2007$ & 0.8 & 0.9 & 0.8 & 0.8 & 0.8 & 0.7 & 0.7 & 0.8 & 0.9 \\
\hline $3 / 28 / 2007$ & 1.9 & 2.9 & 2.3 & 2.2 & 2.3 & 2.2 & 2.1 & 2.1 & 2.6 \\
\hline $4 / 24 / 2007$ & 1.7 & 2.2 & 2.7 & 2.3 & 2.3 & 2.5 & 2.3 & 2.3 & 2.2 \\
\hline $5 / 9 / 2007$ & 1.4 & 1.7 & 2.3 & 1.7 & 1.7 & 2.1 & 1.9 & 1.8 & 1.4 \\
\hline $5 / 23 / 2007$ & 1.5 & 1.8 & 2.2 & 1.5 & 1.8 & 1.8 & 1.7 & 1.7 & 1.7 \\
\hline $6 / 27 / 2007$ & 1.7 & 1.7 & 2.0 & 1.6 & 1.7 & 1.5 & 1.4 & 1.4 & 1.2 \\
\hline $7 / 4 / 2007$ & 1.8 & 1.8 & 2.0 & 1.6 & 1.5 & & & & 0.6 \\
\hline $7 / 18 / 2007$ & 1.5 & 1.3 & 1.4 & 1.1 & 1.0 & 1.1 & 1.0 & 0.9 & 0.6 \\
\hline $8 / 15 / 2007$ & 1.8 & 1.7 & 2.3 & 1.4 & 1.4 & 1.3 & 1.1 & 1.1 & 0.7 \\
\hline $9 / 19 / 2007$ & 1.2 & 1.1 & 1.4 & 1.0 & 1.0 & & & & 0.5 \\
\hline Average & 1.2 & 1.3 & 1.4 & 1.2 & 1.2 & 1.2 & 1.1 & 1.1 & 1.0 \\
\hline $\begin{array}{l}\text { Standard } \\
\text { Deviation }\end{array}$ & 0.5 & 0.6 & 0.8 & 0.6 & 0.6 & 0.7 & 0.7 & 0.6 & 0.6 \\
\hline $\begin{array}{l}\text { Sampling } \\
\text { Date }\end{array}$ & $\begin{array}{c}\text { Point } \\
1\end{array}$ & $\begin{array}{c}\text { Point } \\
1-3\end{array}$ & $\begin{array}{l}\text { OW1- } \\
3-6 a_{B}\end{array}$ & $\begin{array}{l}\text { OW1- } \\
3-6 a_{M}\end{array}$ & $\begin{array}{l}\text { OW1- } \\
3-6 a_{s}\end{array}$ & $\begin{array}{l}\text { OW1- } \\
3-6 b_{B}\end{array}$ & $\begin{array}{l}\text { OW1- } \\
\text { 3-6b }\end{array}$ & $\begin{array}{l}\text { OW1- } \\
3-6 b_{S}\end{array}$ & $\begin{array}{c}\text { Point } \\
\text { 3-6 }\end{array}$ \\
\hline $10 / 19 / 2006$ & 0.6 & 0.7 & 0.7 & 0.6 & 0.7 & 0.4 & 0.4 & 0.4 & 0.3 \\
\hline $11 / 6 / 2006$ & 0.7 & 0.7 & 0.5 & 0.5 & 0.5 & 0.5 & 0.5 & 0.4 & 0.3 \\
\hline $12 / 19 / 2006$ & 0.8 & 0.8 & 0.7 & 0.7 & 0.6 & 0.7 & 0.7 & 0.6 & 0.7 \\
\hline $1 / 8 / 2007$ & 0.8 & 0.9 & 0.8 & 0.8 & 0.8 & 0.7 & 0.7 & 0.7 & 0.8 \\
\hline $1 / 17 / 2007$ & 0.7 & 0.7 & 0.7 & 0.7 & 0.7 & 0.7 & 0.7 & 0.7 & 0.8 \\
\hline $1 / 24 / 2007$ & 0.7 & 0.7 & 0.8 & 0.7 & 0.7 & 0.7 & 0.7 & 0.7 & 0.8 \\
\hline $1 / 30 / 2007$ & 0.7 & 0.8 & 0.7 & 0.7 & 0.8 & 0.8 & 0.7 & 0.8 & 0.8 \\
\hline $2 / 7 / 2007$ & 0.8 & 0.9 & 0.8 & 0.8 & 0.7 & 0.8 & 0.8 & 0.7 & 0.9 \\
\hline $3 / 28 / 2007$ & 1.9 & 2.9 & 2.2 & 2.1 & 2.1 & 2.1 & 2.1 & 2.1 & 2.2 \\
\hline $4 / 24 / 2007$ & 1.7 & 2.1 & 2.3 & 2.0 & 2.0 & 2.3 & 1.9 & 1.9 & 1.7 \\
\hline $5 / 9 / 2007$ & 1.4 & 1.7 & 1.9 & 1.7 & 1.6 & 2.3 & 1.4 & 1.4 & 1.0 \\
\hline $5 / 23 / 2007$ & 1.5 & 1.7 & 1.8 & 1.6 & 1.7 & 2.0 & 1.6 & 1.7 & 1.5 \\
\hline $6 / 27 / 2007$ & 1.7 & 1.6 & 1.6 & 1.5 & 1.5 & 1.3 & 1.2 & 1.2 & 1.1 \\
\hline $7 / 4 / 2007$ & 1.8 & 1.7 & 1.9 & 1.4 & 1.5 & & & & 0.9 \\
\hline $7 / 18 / 2007$ & 1.5 & 1.3 & 1.2 & 1.3 & 1.1 & 1.3 & 1.2 & 1.2 & 0.8 \\
\hline $8 / 15 / 2007$ & 1.8 & 1.6 & 1.4 & 1.4 & 1.4 & 1.3 & 1.1 & 1.0 & 0.8 \\
\hline $9 / 19 / 2007$ & 1.2 & 1.1 & 1.2 & 1.0 & 1.0 & & & & 0.6 \\
\hline
\end{tabular}




\begin{tabular}{|c|c|c|c|c|c|c|c|c|c|}
\hline Average & 1.2 & 1.3 & 1.2 & 1.1 & 1.1 & 1.2 & 1.0 & 1.0 & 0.9 \\
\hline $\begin{array}{l}\text { Standard } \\
\text { Deviation }\end{array}$ & 0.5 & 0.6 & 0.6 & 0.5 & 0.5 & 0.7 & 0.5 & 0.5 & 0.5 \\
\hline $\begin{array}{l}\text { Sampling } \\
\text { Date }\end{array}$ & $\begin{array}{c}\text { Point } \\
1\end{array}$ & $\begin{array}{l}\text { Point } \\
1-4\end{array}$ & $\begin{array}{l}\text { OW1- } \\
4-7 \mathbf{a}_{B}\end{array}$ & $\begin{array}{l}\text { OW1- } \\
4-7 a_{M}\end{array}$ & $\begin{array}{l}\text { OW1- } \\
4-7 a_{s}\end{array}$ & $\begin{array}{l}\text { OW1- } \\
4-7 b_{B}\end{array}$ & $\begin{array}{l}\text { OW1- } \\
4-7 b_{M}\end{array}$ & $\begin{array}{l}\text { OW1- } \\
4-7 b_{S}\end{array}$ & $\begin{array}{c}\text { Point } \\
4-7\end{array}$ \\
\hline $10 / 19 / 2006$ & 0.6 & 0.6 & 0.6 & 0.6 & 0.6 & 0.3 & 0.3 & 0.4 & 0.3 \\
\hline $11 / 6 / 2006$ & 0.7 & 0.6 & 0.5 & 0.43 & 0.5 & 0.3 & 0.3 & 0.4 & 0.4 \\
\hline $12 / 19 / 2006$ & 0.8 & 0.8 & 0.7 & 0.7 & 0.7 & 0.7 & 0.7 & 0.6 & 0.6 \\
\hline $1 / 8 / 2007$ & 0.8 & 0.9 & 0.7 & 0.8 & 0.8 & 0.7 & 0.7 & 0.7 & 0.8 \\
\hline $1 / 17 / 2007$ & 0.7 & 0.7 & 0.8 & 0.7 & 0.8 & 0.7 & 0.7 & 0.8 & 0.8 \\
\hline $1 / 24 / 2007$ & 0.7 & 0.7 & 0.8 & 0.7 & 0.7 & 0.8 & 0.7 & 0.8 & 0.8 \\
\hline $1 / 30 / 2007$ & 0.7 & 0.7 & 0.8 & 0.7 & 0.7 & 0.7 & 0.7 & 0.7 & 0.8 \\
\hline $2 / 7 / 2007$ & 0.8 & 0.8 & 0.8 & 0.8 & 0.8 & 0.7 & 0.8 & 0.8 & 0.9 \\
\hline $3 / 28 / 2007$ & 1.9 & 3.5 & 2.2 & 2.1 & 2.2 & 2.1 & 2.1 & 2.1 & 2.4 \\
\hline $4 / 24 / 2007$ & 1.7 & 2.1 & 2.8 & 2.1 & 2.2 & 2.4 & 2.2 & 2.2 & 2.1 \\
\hline $5 / 9 / 2007$ & 1.4 & 1.5 & 1.9 & 1.5 & 1.5 & 2.3 & 1.6 & 1.6 & 1.5 \\
\hline $5 / 23 / 2007$ & 1.5 & 1.7 & 1.6 & 1.5 & 1.6 & 1.8 & 1.6 & 1.6 & 1.7 \\
\hline $6 / 27 / 2007$ & 1.7 & 1.6 & 1.6 & 1.4 & 1.5 & 1.2 & 1.0 & 1.0 & 1.1 \\
\hline $7 / 4 / 2007$ & 1.8 & 1.4 & 1.4 & 1.3 & 1.4 & & & & 0.7 \\
\hline $7 / 18 / 2007$ & 1.5 & 1.2 & 1.5 & 0.9 & 0.8 & 0.8 & 0.7 & 0.7 & 0.7 \\
\hline $8 / 15 / 2007$ & 1.8 & 1.6 & 0.7 & 0.7 & 0.7 & 0.7 & 1.2 & 1.2 & 0.7 \\
\hline 9/19/2007 & 1.2 & 1.1 & 1.0 & 0.9 & 0.9 & & & & 0.5 \\
\hline Average & 1.2 & 1.3 & 1.2 & 1.1 & 1.1 & 1.1 & 1.0 & 1.0 & 1.0 \\
\hline $\begin{array}{l}\text { Standard } \\
\text { Deviation }\end{array}$ & 0.5 & 0.7 & 0.7 & 0.5 & 0.5 & 0.7 & 0.6 & 0.6 & 0.6 \\
\hline
\end{tabular}




\begin{tabular}{|c|c|c|c|c|c|c|c|c|c|}
\hline Date & $\begin{array}{c}\text { Point } \\
1\end{array}$ & $\begin{array}{l}\text { Point } \\
1-2\end{array}$ & $\begin{array}{l}\text { OW1- } \\
2-5 a_{B}\end{array}$ & $\begin{array}{l}\text { OW1- } \\
2-5 a_{M}\end{array}$ & $\begin{array}{l}\text { OW1- } \\
2-5 a_{S}\end{array}$ & $\begin{array}{l}\text { OW1- } \\
2-5 b_{B}\end{array}$ & $\begin{array}{l}\text { OW1- } \\
2-5 b_{M}\end{array}$ & $\begin{array}{l}\text { OW1- } \\
2-5 b_{S}\end{array}$ & $\begin{array}{c}\text { Point } \\
2-5\end{array}$ \\
\hline $10 / 19 / 2006$ & 0.3 & 0.3 & 0.3 & 0.3 & 0.3 & 0.1 & 0.1 & 0.2 & 0.1 \\
\hline $11 / 6 / 2006$ & 0.3 & 0.3 & 0.2 & 0.2 & 0.2 & 0.2 & 0.2 & 0.2 & 0.2 \\
\hline $12 / 19 / 2006$ & 0.4 & 0.4 & 0.3 & 0.3 & 0.3 & 0.3 & 0.3 & 0.2 & 0.2 \\
\hline $1 / 8 / 2007$ & 0.4 & 0.4 & 0.6 & 0.4 & 0.4 & 0.4 & 0.4 & 0.4 & 0.4 \\
\hline $1 / 17 / 2007$ & 0.4 & 0.4 & 0.4 & 0.4 & 0.4 & 0.4 & 0.4 & 0.4 & 0.4 \\
\hline $1 / 24 / 2007$ & 0.4 & 0.4 & 0.4 & 0.4 & 0.4 & 0.4 & 0.4 & 0.4 & 0.4 \\
\hline $1 / 30 / 2007$ & 0.4 & 0.4 & 0.4 & 0.4 & 0.4 & 0.4 & 0.4 & 0.4 & 0.4 \\
\hline $2 / 7 / 2007$ & 0.4 & 0.5 & 0.4 & 0.4 & 0.4 & 0.4 & 0.4 & 0.4 & 0.4 \\
\hline $3 / 28 / 2007$ & 0.9 & 1.5 & 1.3 & 1.2 & 1.2 & 1.2 & 1.2 & 1.2 & 1.3 \\
\hline $4 / 24 / 2007$ & 0.9 & 1.1 & 1.4 & 1.2 & 1.2 & 1.3 & 1.2 & 1.2 & 1.1 \\
\hline $5 / 9 / 2007$ & 0.7 & 0.8 & 1.2 & 0.9 & 0.9 & 1.1 & 1.0 & 0.9 & 0.7 \\
\hline $5 / 23 / 2007$ & 0.7 & 0.9 & 1.2 & 0.8 & 0.9 & 1.0 & 0.9 & 0.9 & 0.8 \\
\hline $6 / 27 / 2007$ & 0.8 & 0.8 & 1.0 & 0.8 & 0.8 & 0.8 & 0.7 & 0.7 & 0.6 \\
\hline $7 / 4 / 2007$ & 0.9 & 0.8 & 1.0 & 0.8 & 0.7 & & & & 0.3 \\
\hline $7 / 18 / 2007$ & 0.7 & 0.6 & 0.7 & 0.5 & 0.5 & 0.6 & 0.5 & 0.4 & 0.3 \\
\hline $8 / 15 / 2007$ & 0.9 & 0.8 & 1.2 & 0.7 & 0.7 & 0.6 & 0.6 & 0.6 & 0.4 \\
\hline 9/19/2007 & 0.6 & 0.6 & 0.7 & 0.5 & 0.5 & & & & 0.3 \\
\hline Average & 0.6 & 0.6 & 0.7 & 0.6 & 0.6 & 0.6 & 0.6 & 0.6 & 0.5 \\
\hline $\begin{array}{l}\text { Standard } \\
\text { Deviation }\end{array}$ & 0.2 & 0.3 & 0.4 & 0.3 & 0.3 & 0.4 & 0.4 & 0.3 & 0.3 \\
\hline $\begin{array}{l}\text { Sampling } \\
\text { Date }\end{array}$ & $\begin{array}{c}\text { Point } \\
1\end{array}$ & $\begin{array}{c}\text { Point } \\
1-3\end{array}$ & $\begin{array}{l}\text { OW1- } \\
3-6 a_{B}\end{array}$ & $\begin{array}{l}\text { OW1- } \\
3-6 a_{M}\end{array}$ & $\begin{array}{l}\text { OW1- } \\
3-6 a_{s}\end{array}$ & $\begin{array}{l}\text { OW1- } \\
3-6 b_{B}\end{array}$ & $\begin{array}{l}\text { OW1- } \\
\text { 3-6b }\end{array}$ & $\begin{array}{l}\text { OW1- } \\
3-6 b_{S}\end{array}$ & $\begin{array}{c}\text { Point } \\
\text { 3-6 }\end{array}$ \\
\hline $10 / 19 / 2006$ & 0.3 & 0.3 & 0.3 & 0.3 & 0.3 & 0.2 & 0.2 & 0.2 & 0.1 \\
\hline $11 / 6 / 2006$ & 0.3 & 0.3 & 0.3 & 0.2 & 0.2 & 0.2 & 0.2 & 0.2 & 0.2 \\
\hline $12 / 19 / 2006$ & 0.4 & 0.4 & 0.4 & 0.3 & 0.3 & 0.3 & 0.3 & 0.3 & 0.3 \\
\hline $1 / 8 / 2007$ & 0.4 & 0.4 & 0.4 & 0.4 & 0.4 & 0.4 & 0.4 & 0.4 & 0.4 \\
\hline $1 / 17 / 2007$ & 0.4 & 0.4 & 0.4 & 0.4 & 0.4 & 0.4 & 0.4 & 0.4 & 0.4 \\
\hline $1 / 24 / 2007$ & 0.4 & 0.4 & 0.4 & 0.4 & 0.4 & 0.4 & 0.4 & 0.4 & 0.4 \\
\hline $1 / 30 / 2007$ & 0.4 & 0.4 & 0.4 & 0.4 & 0.4 & 0.4 & 0.4 & 0.4 & 0.4 \\
\hline $2 / 7 / 2007$ & 0.4 & 0.5 & 0.4 & 0.4 & 0.4 & 0.4 & 0.4 & 0.4 & 0.4 \\
\hline $3 / 28 / 2007$ & 0.9 & 1.4 & 1.3 & 1.2 & 1.2 & 1.2 & 1.1 & 1.1 & 1.1 \\
\hline $4 / 24 / 2007$ & 0.9 & 1.0 & 1.2 & 1.0 & 1.0 & 1.2 & 1.0 & 1.0 & 0.8 \\
\hline $5 / 9 / 2007$ & 0.7 & 0.8 & 1.0 & 0.8 & 0.8 & 1.2 & 0.7 & 0.7 & 0.5 \\
\hline $5 / 23 / 2007$ & 0.7 & 0.8 & 0.9 & 0.9 & 0.9 & 1.0 & 0.8 & 0.8 & 0.8 \\
\hline $6 / 27 / 2007$ & 0.8 & 0.8 & 0.8 & 0.8 & 0.8 & 0.7 & 0.6 & 0.6 & 0.6 \\
\hline $7 / 4 / 2007$ & 0.9 & 0.8 & 0.8 & 0.7 & 0.8 & & & & 0.4 \\
\hline $7 / 18 / 2007$ & 0.7 & 0.6 & 0.6 & 0.6 & 0.6 & 0.7 & 0.6 & 0.6 & 0.4 \\
\hline $8 / 15 / 2007$ & 0.9 & 0.8 & 0.7 & 0.7 & 0.7 & 0.6 & 0.6 & 0.5 & 0.4 \\
\hline $9 / 19 / 2007$ & 0.6 & 0.5 & 0.6 & 0.5 & 0.5 & & & & 0.3 \\
\hline
\end{tabular}




\begin{tabular}{|c|c|c|c|c|c|c|c|c|c|}
\hline Average & 0.6 & 0.6 & 0.6 & 0.6 & 0.6 & 0.6 & 0.5 & 0.5 & 0.5 \\
\hline $\begin{array}{l}\text { Standard } \\
\text { Deviation }\end{array}$ & 0.2 & 0.3 & 0.3 & 0.3 & 0.3 & 0.4 & 0.3 & 0.3 & 0.2 \\
\hline $\begin{array}{c}\text { Sampling } \\
\text { Date }\end{array}$ & $\begin{array}{c}\text { Point } \\
1\end{array}$ & $\begin{array}{c}\text { Point } \\
1-4\end{array}$ & $\begin{array}{l}\text { OW1- } \\
4-7 \mathbf{a}_{B}\end{array}$ & $\begin{array}{l}\text { OW1- } \\
4-7 a_{M}\end{array}$ & $\begin{array}{l}\text { OW1- } \\
4-7 a_{s}\end{array}$ & $\begin{array}{l}\text { OW1- } \\
4-7 b_{B}\end{array}$ & $\begin{array}{l}\text { OW1- } \\
4-7 b_{M}\end{array}$ & $\begin{array}{l}\text { OW1- } \\
4-7 b_{s}\end{array}$ & $\begin{array}{c}\text { Point } \\
4-7\end{array}$ \\
\hline $10 / 19 / 2006$ & 0.3 & 0.3 & 0.3 & 0.3 & 0.3 & 0.2 & 0.2 & 0.2 & 0.1 \\
\hline $11 / 6 / 2006$ & 0.3 & 0.3 & 0.2 & 0.2 & 0.2 & 0.2 & 0.2 & 0.2 & 0.2 \\
\hline $12 / 19 / 2006$ & 0.4 & 0.4 & 0.4 & 0.4 & 0.3 & 0.3 & 0.3 & 0.3 & 0.3 \\
\hline $1 / 8 / 2007$ & 0.4 & 0.4 & 0.4 & 0.4 & 0.4 & 0.4 & 0.4 & 0.4 & 0.4 \\
\hline $1 / 17 / 2007$ & 0.4 & 0.4 & 0.4 & 0.4 & 0.4 & 0.4 & 0.4 & 0.4 & 0.4 \\
\hline $1 / 24 / 2007$ & 0.4 & 0.4 & 0.4 & 0.4 & 0.4 & 0.4 & 0.4 & 0.4 & 0.4 \\
\hline $1 / 30 / 2007$ & 0.4 & 0.4 & 0.4 & 0.4 & 0.4 & 0.4 & 0.4 & 0.4 & 0.4 \\
\hline $2 / 7 / 2007$ & 0.4 & 0.4 & 0.4 & 0.4 & 0.4 & 0.4 & 0.4 & 0.4 & 0.4 \\
\hline $3 / 28 / 2007$ & 0.9 & 1.7 & 1.2 & 1.2 & 1.2 & 1.2 & 1.2 & 1.2 & 1.1 \\
\hline $4 / 24 / 2007$ & 0.9 & 1.0 & 1.5 & 1.1 & 1.1 & 1.3 & 1.2 & 1.1 & 1.1 \\
\hline $5 / 9 / 2007$ & 0.7 & 0.8 & 1.0 & 0.8 & 0.8 & 1.2 & 0.8 & 0.8 & 0.8 \\
\hline $5 / 23 / 2007$ & 0.7 & 0.8 & 0.8 & 0.8 & 0.8 & 0.9 & 0.8 & 0.9 & 0.9 \\
\hline $6 / 27 / 2007$ & 0.8 & 0.8 & 0.8 & 0.7 & 0.8 & 0.6 & 0.6 & 0.5 & 0.6 \\
\hline $7 / 4 / 2007$ & 0.9 & 0.7 & 0.7 & 0.7 & 0.7 & & & & 0.4 \\
\hline $7 / 18 / 2007$ & 0.7 & 0.6 & 0.7 & 0.4 & 0.4 & 0.6 & 0.1 & 0.2 & 0.3 \\
\hline $8 / 15 / 2007$ & 0.9 & 0.7 & 0.3 & 0.3 & 0.3 & 0.3 & 0.6 & 0.6 & 0.3 \\
\hline $9 / 19 / 2007$ & 0.6 & 0.5 & 0.5 & 0.4 & 0.4 & & & & 0.3 \\
\hline Average & 0.6 & 0.6 & 0.6 & 0.5 & 0.5 & 0.6 & 0.5 & 0.5 & 0.5 \\
\hline $\begin{array}{l}\text { Standard } \\
\text { Deviation }\end{array}$ & 0.2 & 0.4 & 0.4 & 0.3 & 0.3 & 0.4 & 0.3 & 0.3 & 0.3 \\
\hline
\end{tabular}




\section{APPENDIX C}

SENSITIVITY ANALYSIS RESULTS 

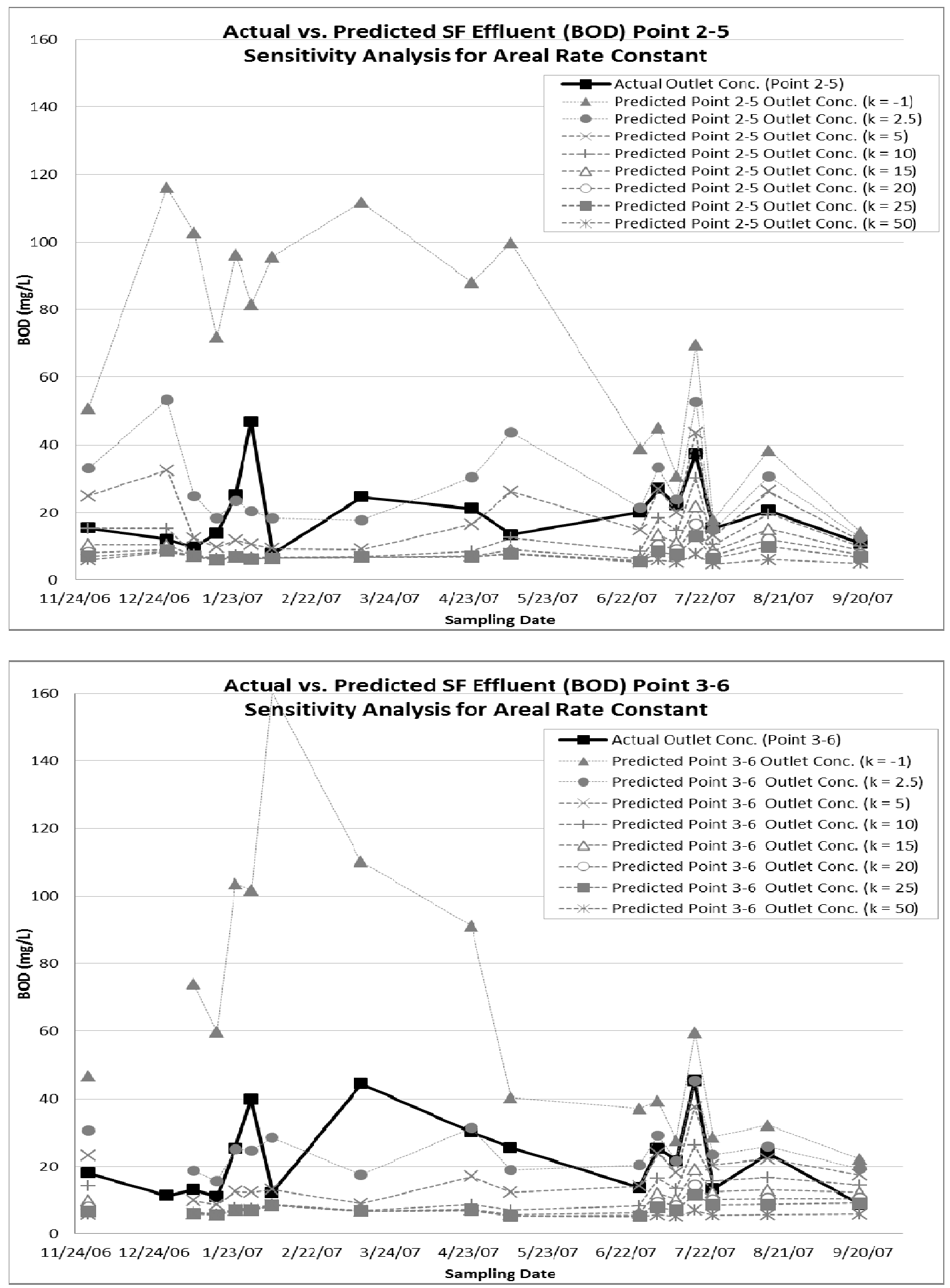

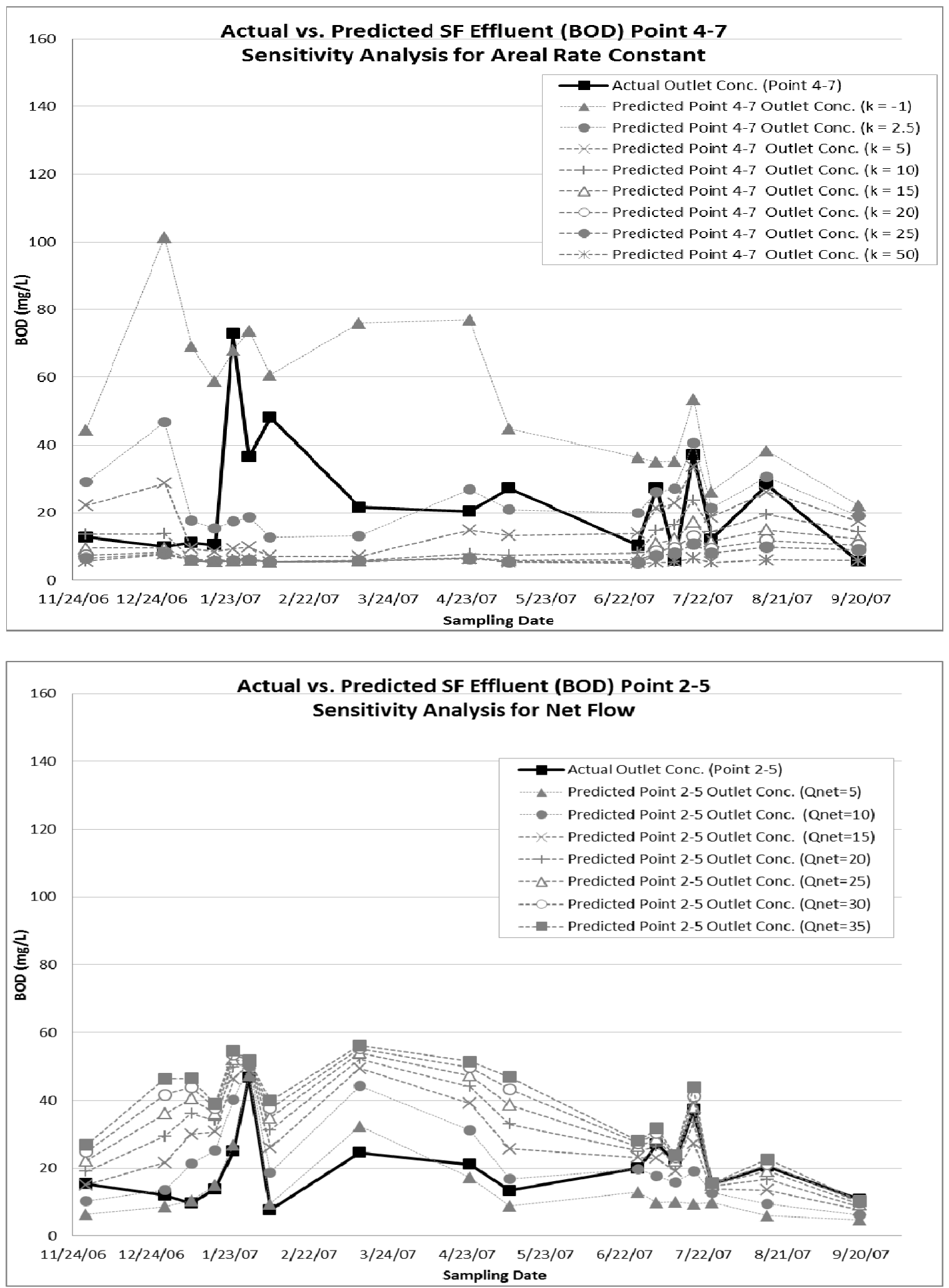

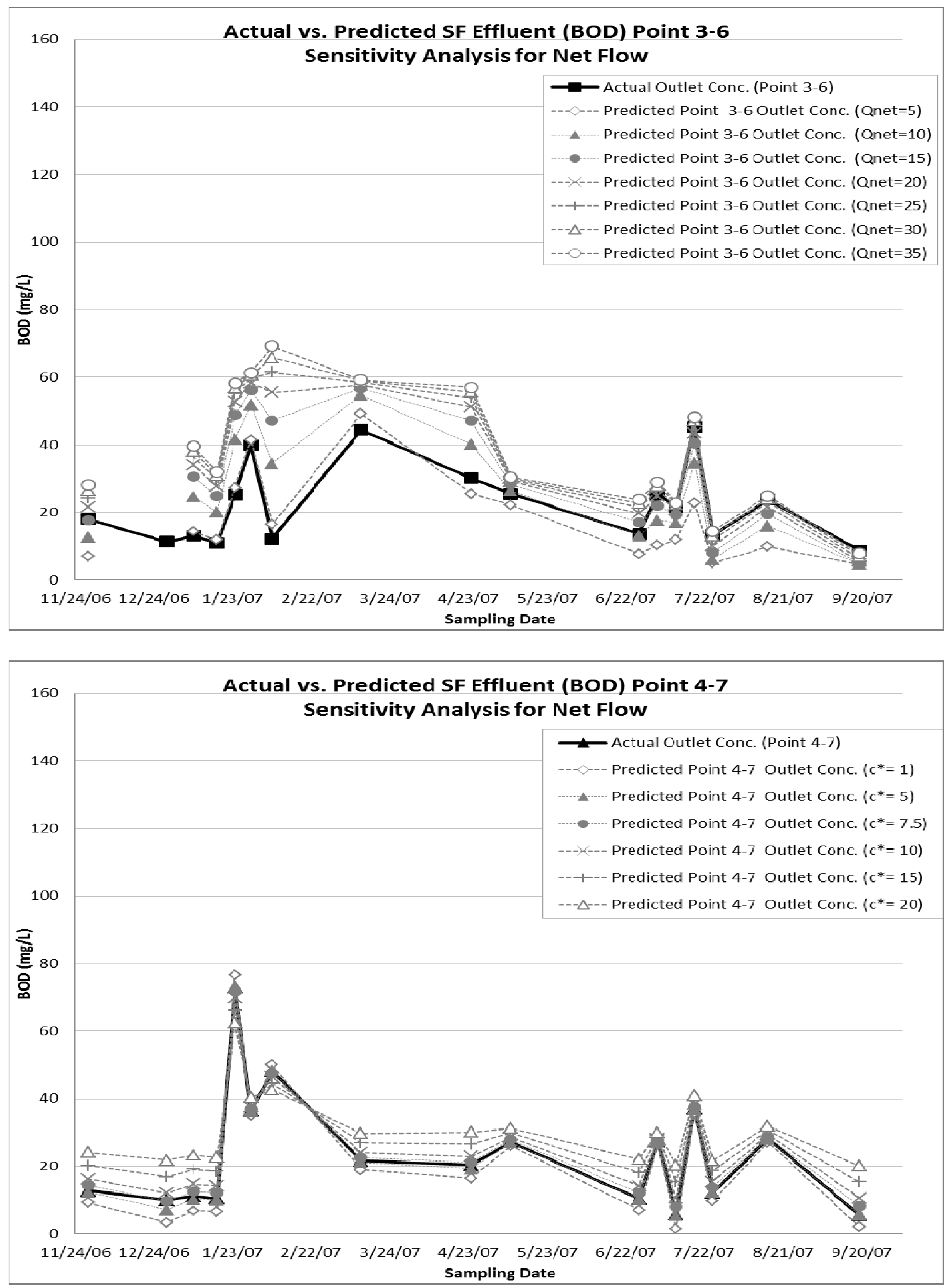

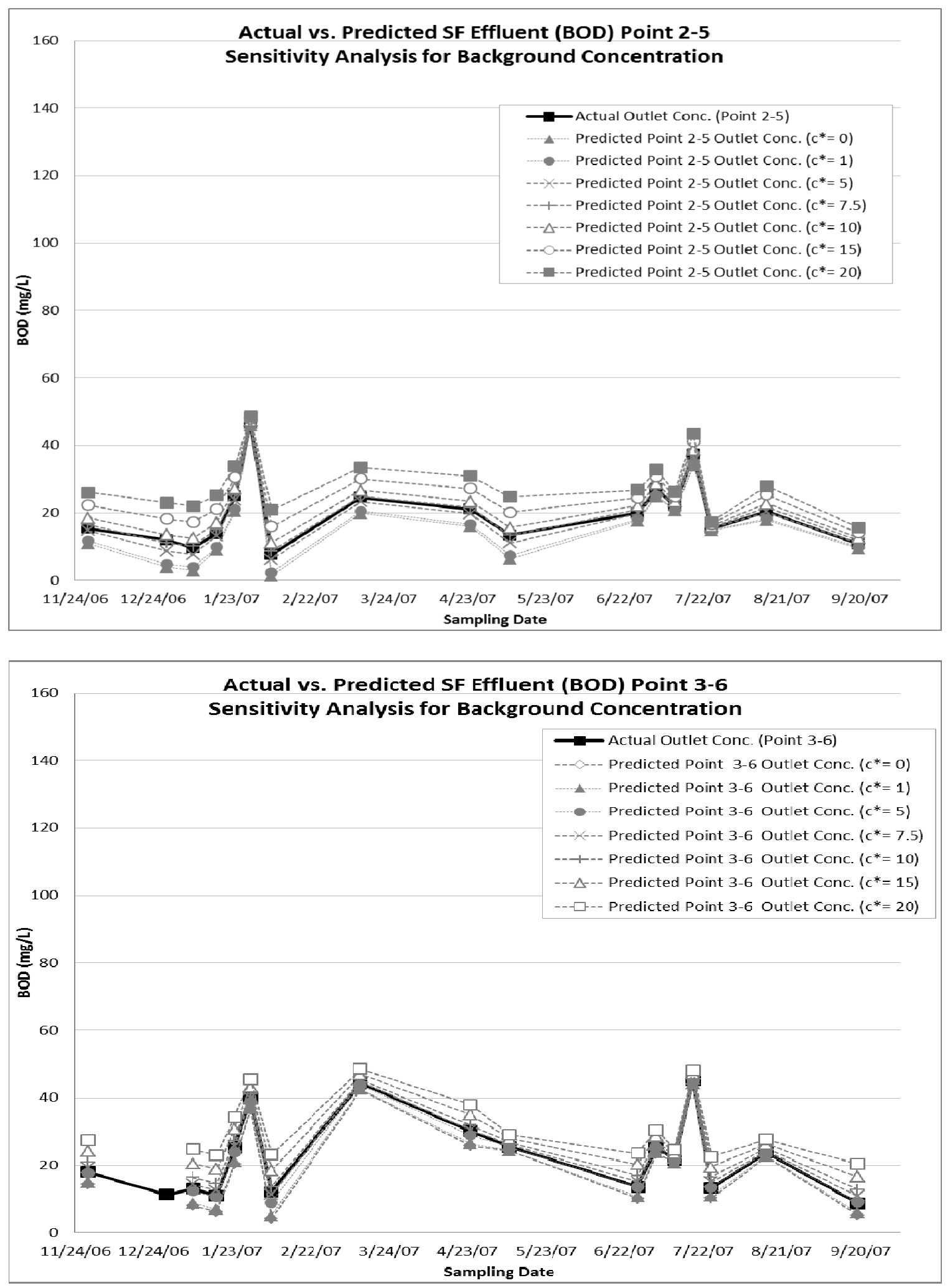

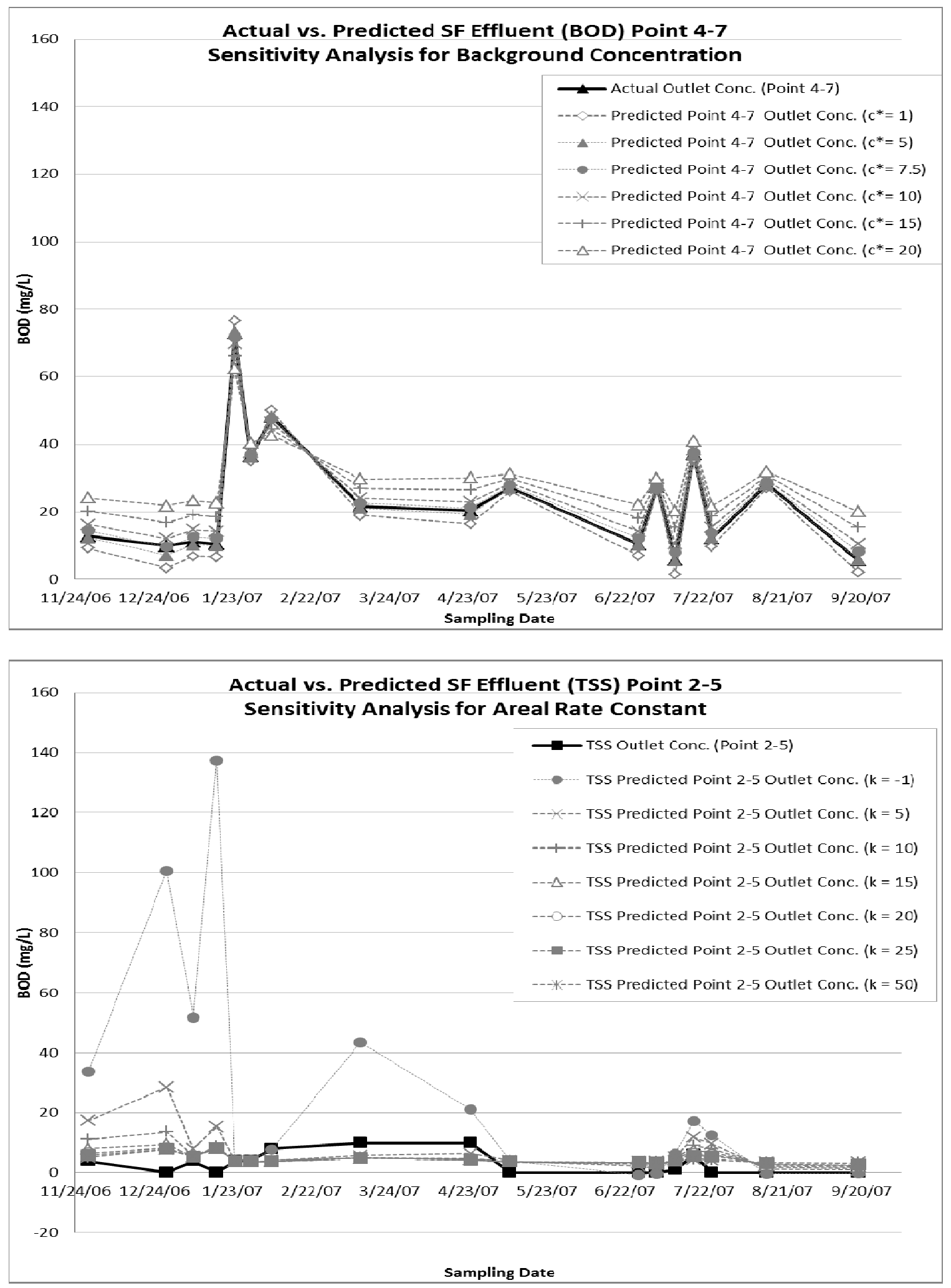

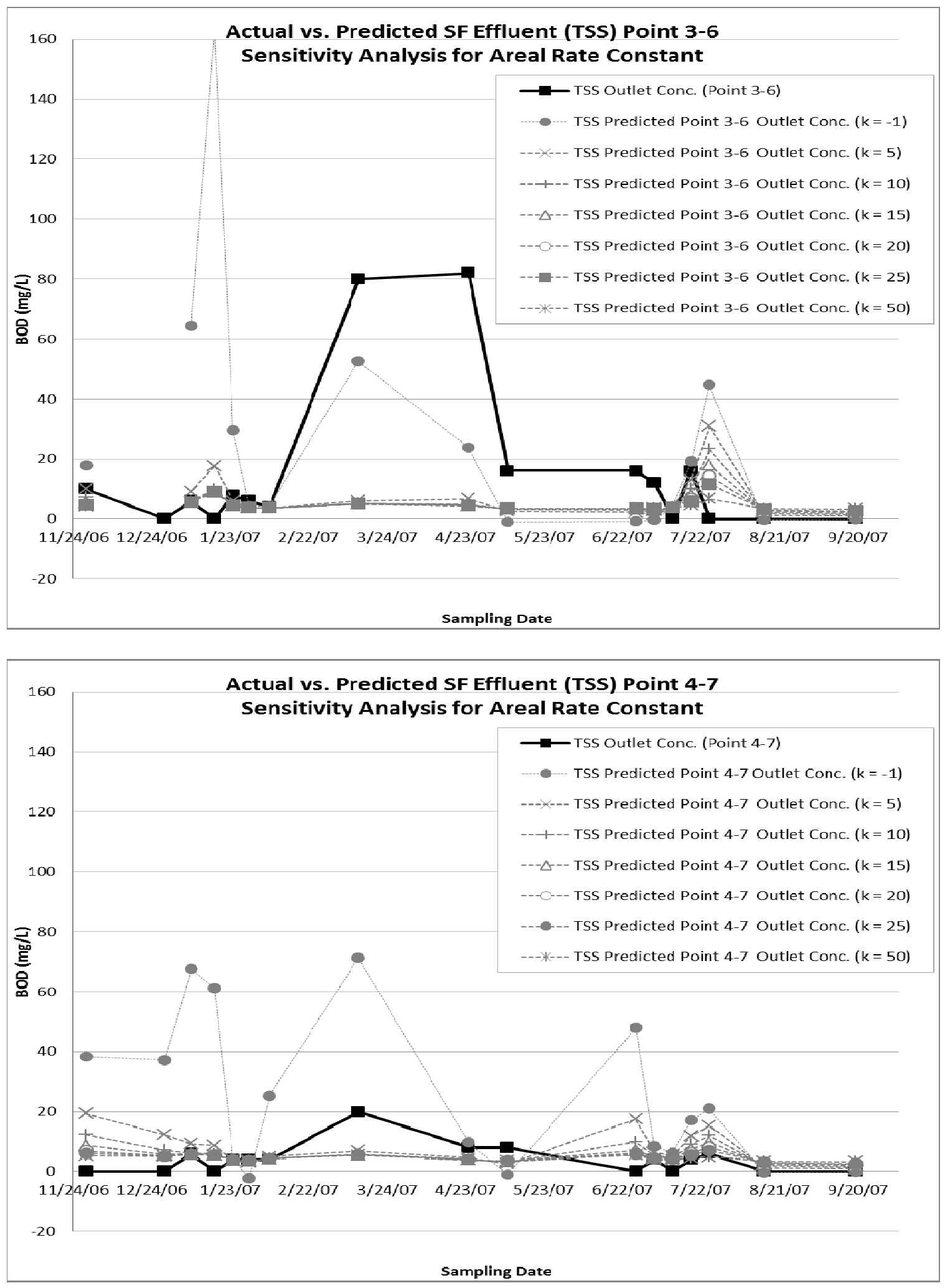

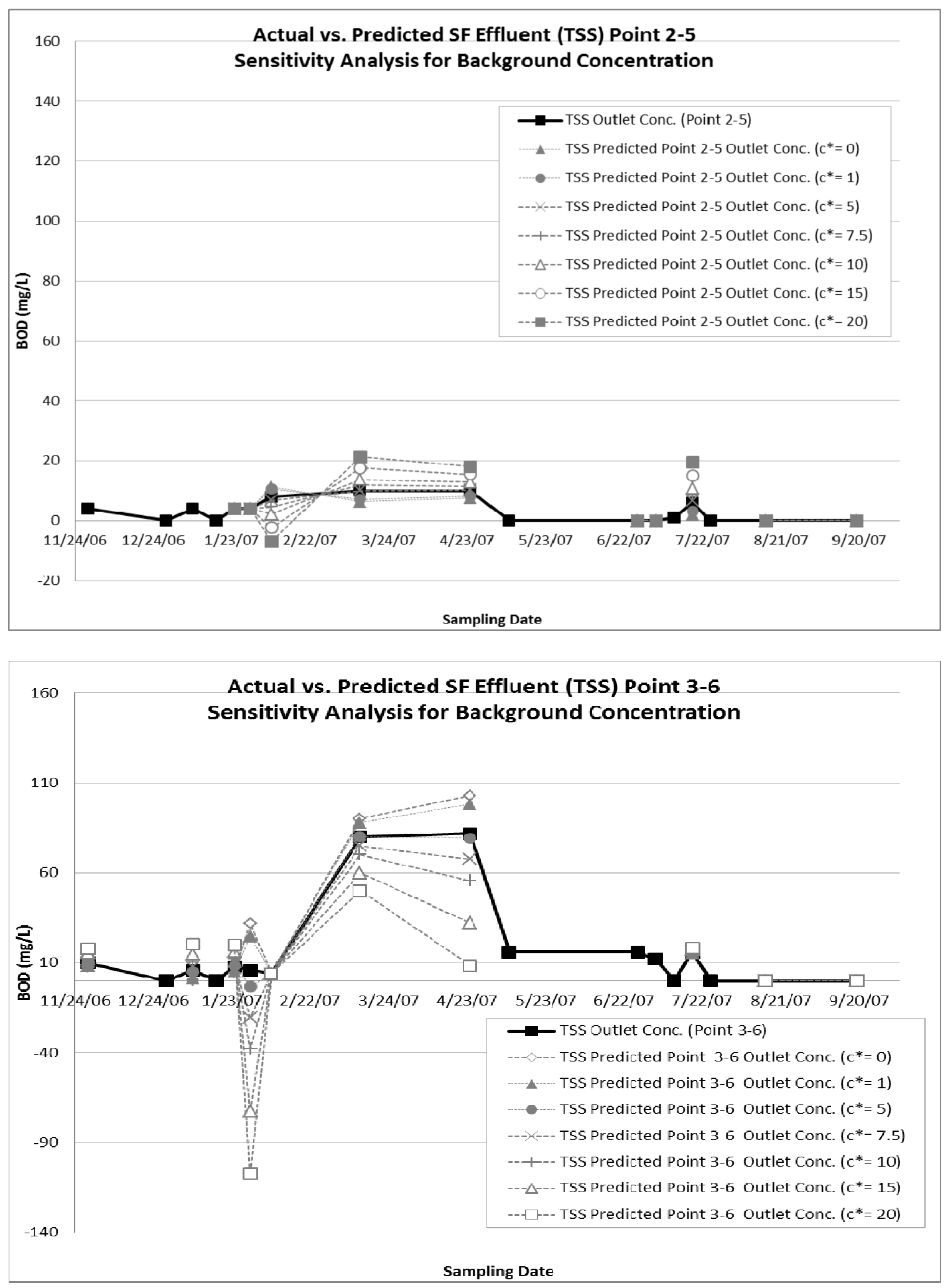

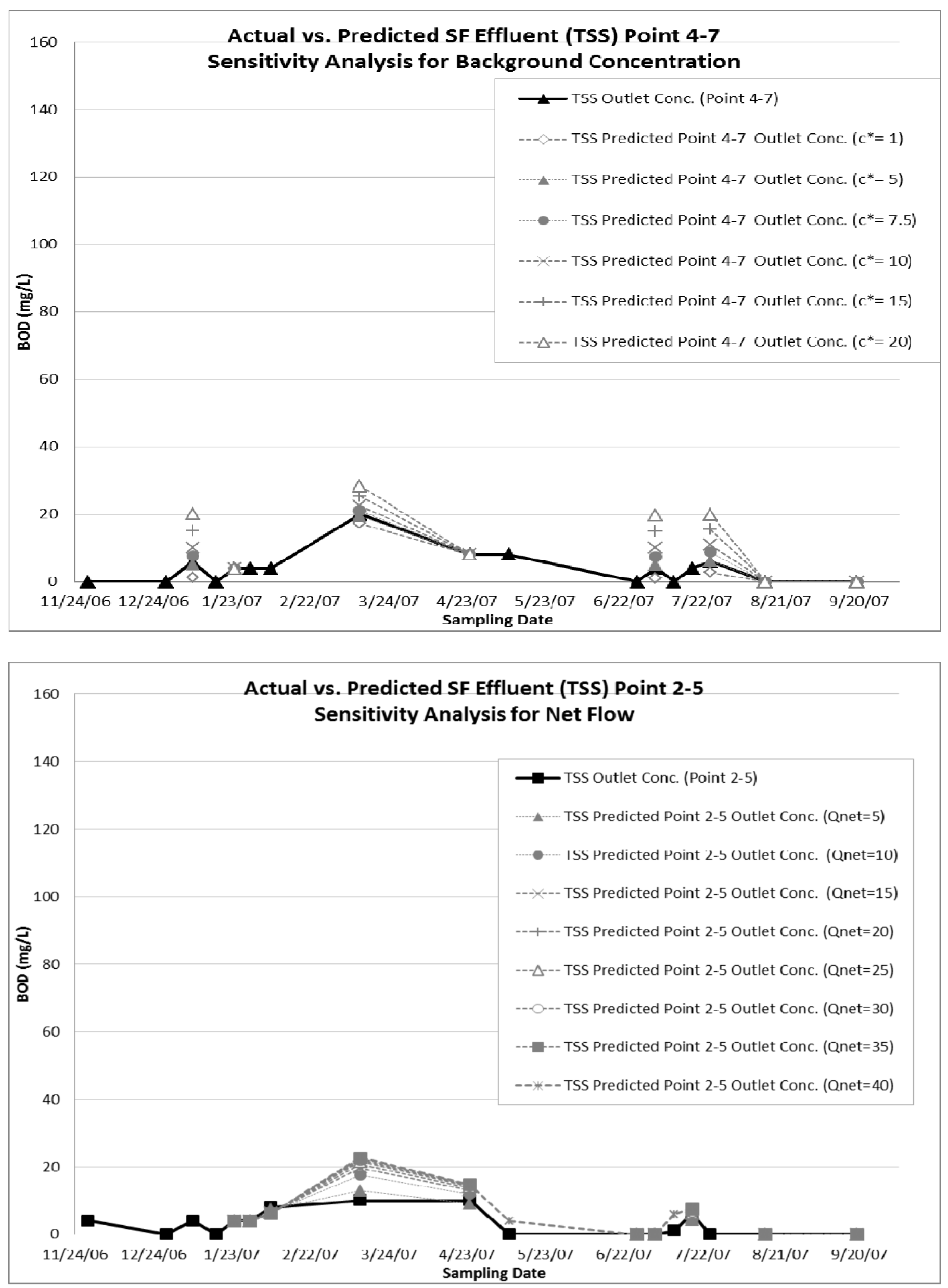

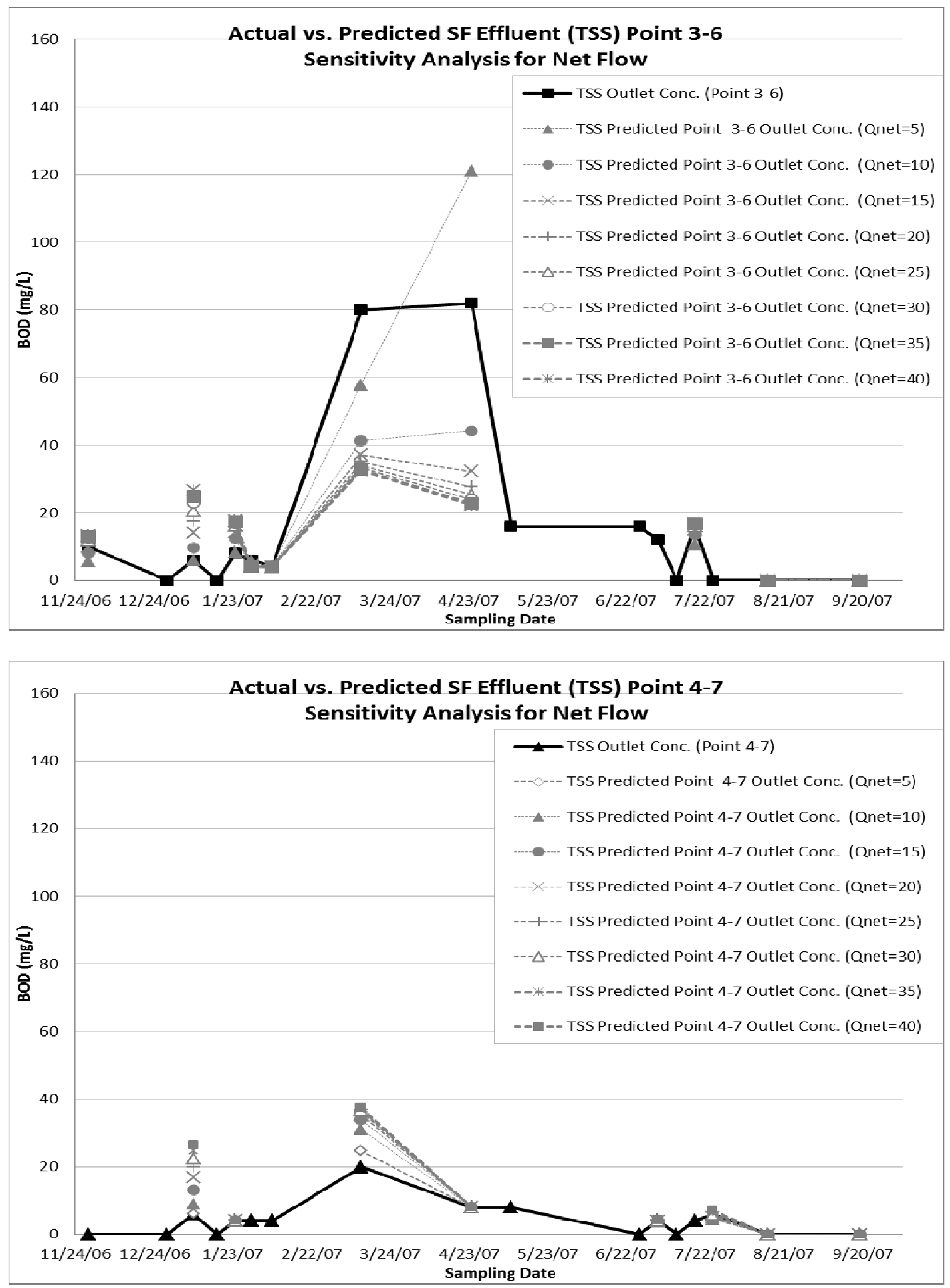
VITA

\section{ADELAIDE PEREIRA HUMMEL}

July 27, 1973 Born, Manaus, Amazonas, Brazil

1998

B.S., Civil Engineering

Universidade de Taubaté

Taubaté, São Paulo, Brazil

1996-1999 Facilities Engineer

Delphi Automotive System

Paraisópolis, Minas Gerais, Brazil

2000-2002 Teaching Assistant

University of Illinois at Springfield

Springfield, Illinois 
M.A., Environmental Studies

University of Illinois at Springfield

Springfield, Illinois

2003-2004 Logistics Analyst

Delphi Automotive System

Paraisópolis, Minas Gerais, Brazil

2005-2006 Research Assistant

Applied Research Center, Florida International University

Miami, Florida

2006-2009 Project Engineer

AECOM USA Ltd. 
Sunrise, Florida

2008

Professional Engineer License. Florida, U.S.A.

2009-current Water and Wastewater Engineer

AECOM Canada Ltd.

Kitchener, Ontario Canada

2014

Professional Engineer License. Ontario, Canada

Publications and Conferences

Hummel Batista, Adelaide P., Nathan Morris, Joseph Gemin, Patricia Quackenbush, Alvin Pilobello, and Zirui (Ray) Yu. Evaluation of Ion Exchange and Reverse Osmosis Technologies for Sulphate Removal from Groundwater for the Region of Waterloo. Ontario Water Works Association Annual Conference, May 2012 (Oral Presentation).

Katsenovich, Yelena P., Adelaide Hummel-Batista, Alfredo J. Ravinet, and Jerry F. Miller, "Performance Evaluation of Constructed Wetlands in a Tropical Region," Ecol Engineering 35 (2009) 1529-1537, 2009.

Stitt, Brian, Creswell, J. H., Hummel, A., and Barnes, S. Sustainable Water Supply for South Florida. American Water Resources Association Annual Conference, November 2007. 